\title{
Electrophilic Reactivities of Vinyl $p$-Quinone Methides
}

\author{
Andreas Eitzinger, ${ }^{1}$ Robert. J. Mayer, ${ }^{2}$ Nathalie Hampel, ${ }^{2}$ Peter Mayer, ${ }^{2}$ \\ Mario Waser, ${ }^{1}$ Armin R. Ofial ${ }^{2, *}$
}

[1] Institute of Organic Chemistry, Johannes Kepler University Linz, Altenberger Straße 69, 4040 Linz (Austria)

[2] Department Chemie, Ludwig-Maximilians-Universität München, Butenandtstraße 5-13, 81377 München (Germany)

ofial@Imu.de

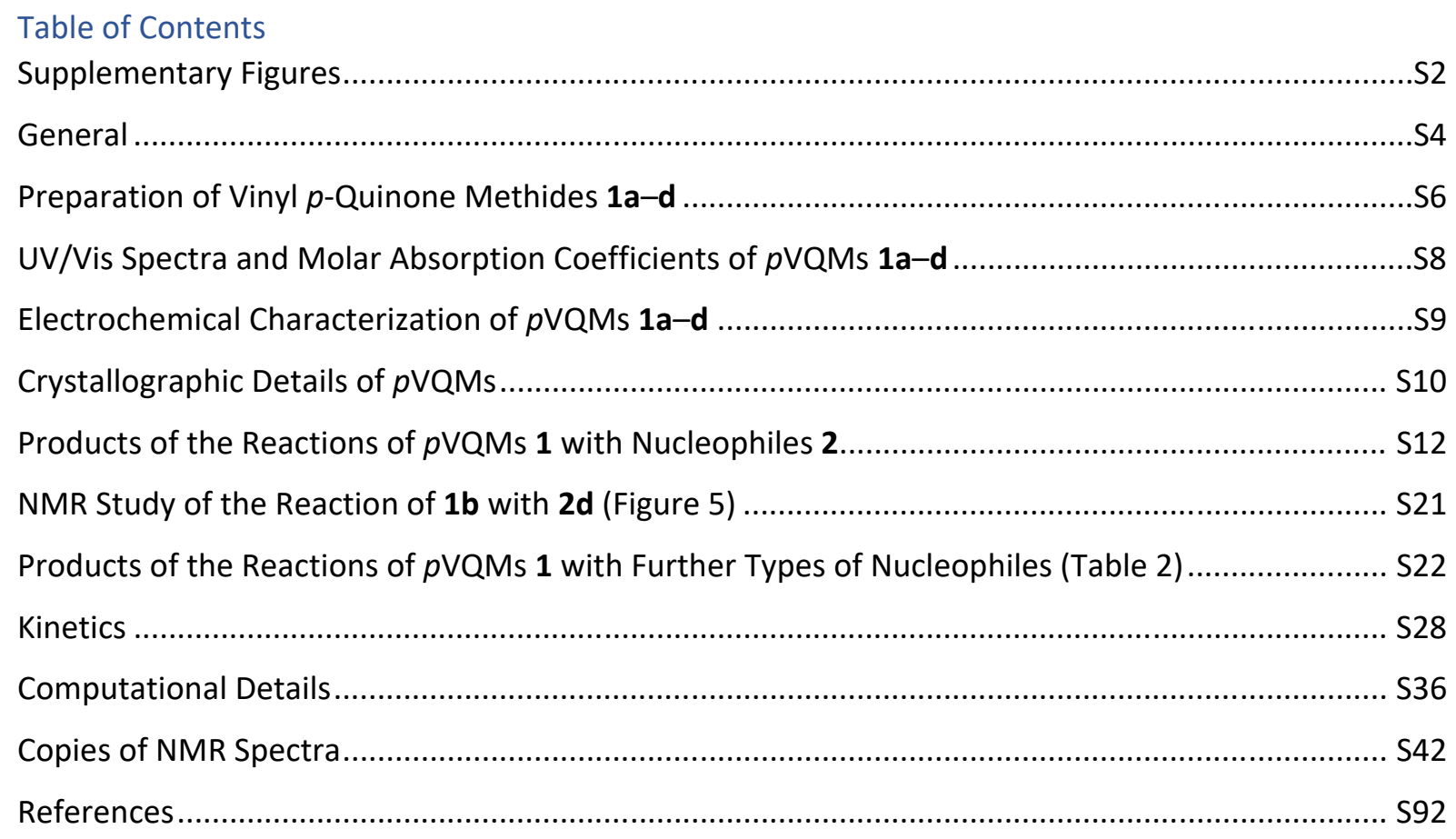




\section{Supplementary Figures}

\section{Determination of Electrophilicity Parameters $E$ for $p$ VQMs}

a)

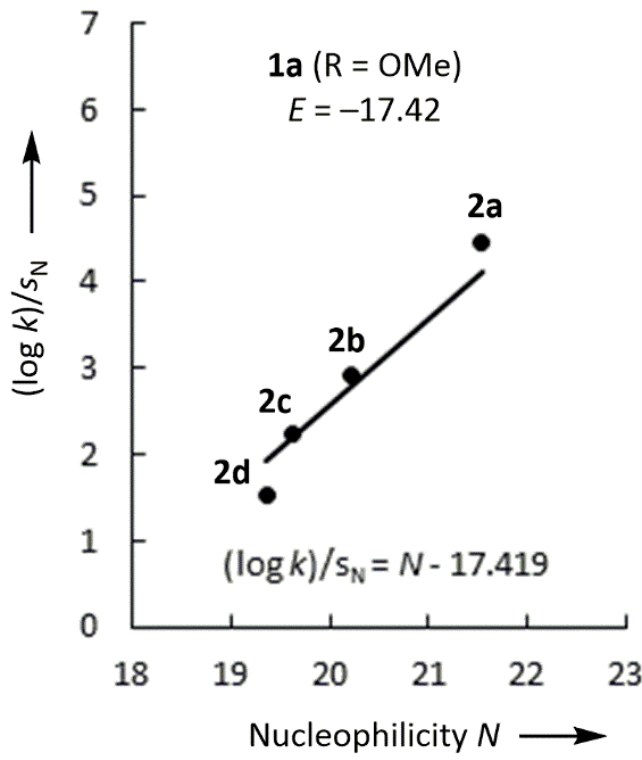

c)

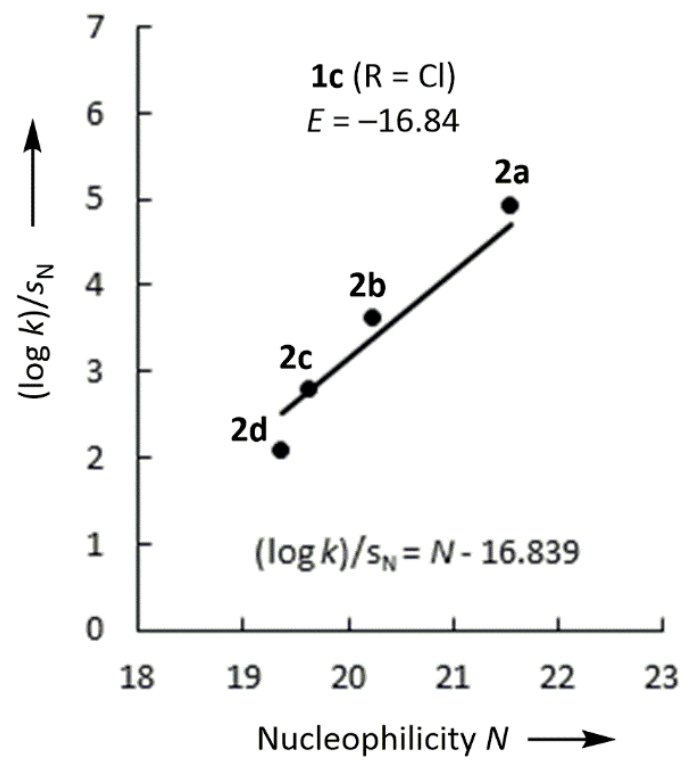

b)

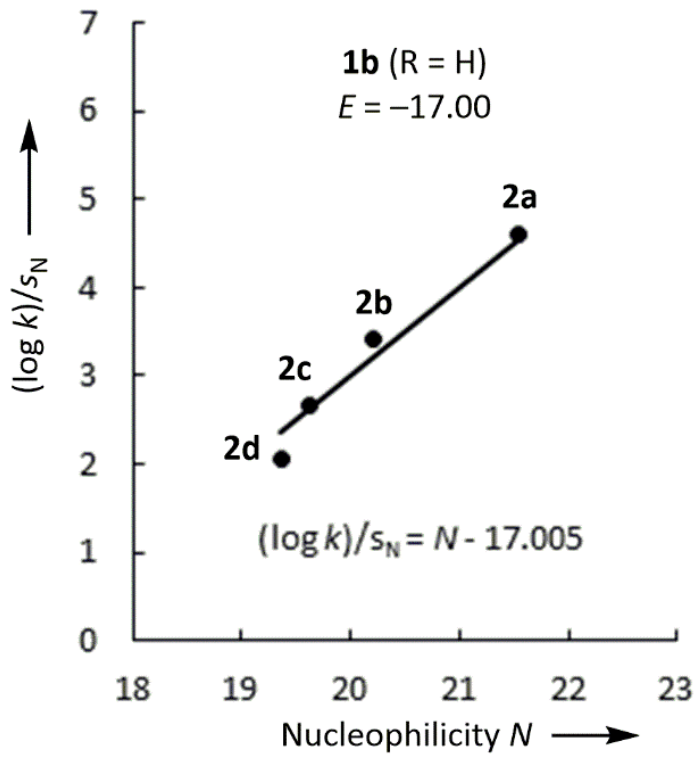

d)

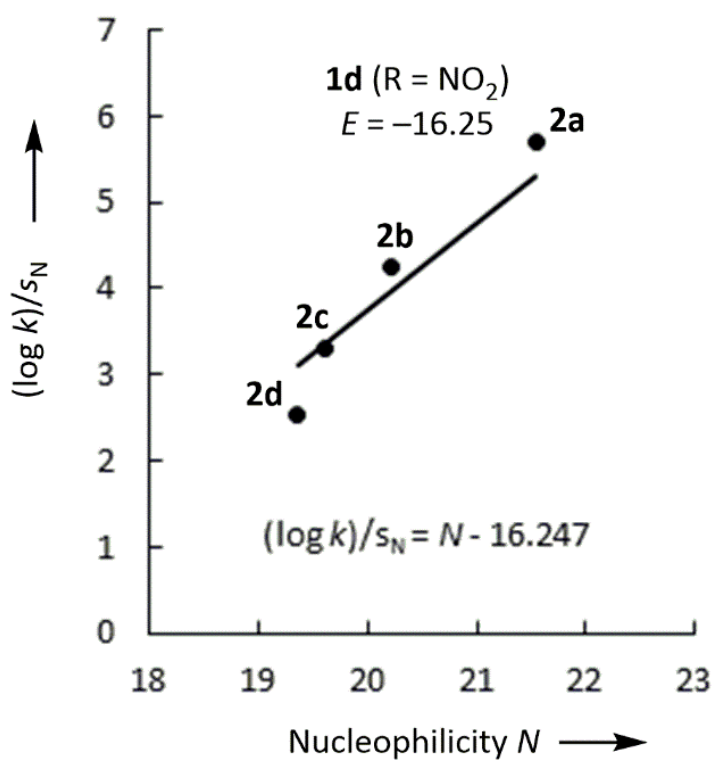

Figure S1. Determination of electrophilicity parameters $E$ for $p$ VQMs $\mathbf{1 a}-\mathbf{d}$. The correlations lines are drawn with a fixed slope of 1 as required by Equation 1 and refer to the result of least-squares analyses that minimize $\Delta^{2}=\left(\log k_{2}{ }^{\exp }-s_{N}(N+E)\right)^{2}$ for each of the $p V Q M s$ (with second-order rate constants in DMSO at $20^{\circ} \mathrm{C}$ from Table 1 , main text, and $N$ and $s_{N}$ parameters of the reference nucleophiles $2 \mathrm{a}-\mathbf{d}$ from Figure 1). 


\section{Hammett plot for $p$ VQMs}

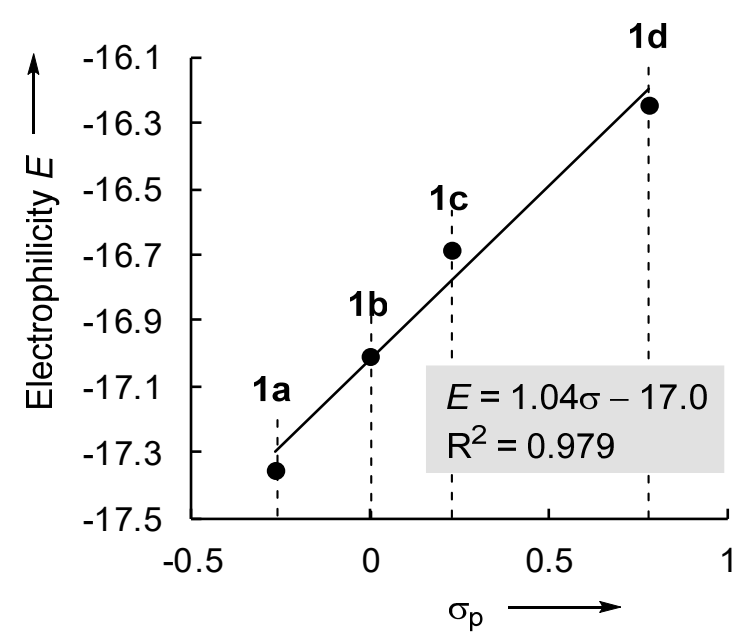

Figure S2. Hammett plot for the electrophilicity parameters $E$ of $p$ VQMs $1 \mathbf{a}-\mathbf{d}$ vs. $\sigma_{\mathrm{p}}{ }^{{ }^{1}}{ }^{1}$ 


\section{General}

Commercial reagents and dry solvents (stored over molecular sieves) were used without further purification as purchased from Sigma-Aldrich or Acros Organics.

For thin-layer chromatography, silica gel plates with F-254 fluorescence indicator (Merck) were used. Purification by flash column chromatography was performed using Merck silica gel 60 (0.040-0.063 $\mathrm{mm}$ ) with freshly distilled solvents. Semi-preparative HPLC was performed using a Thermo Scientific Dionex Ultimate 3000 system with a variable wavelength detector and a normal phase silica column (5 $\mu \mathrm{m}$ particle size, $\mathrm{L} \times I . D .=25 \mathrm{~cm} \times 10 \mathrm{~mm}$ ).

Melting points were acquired using Büchi Melting Point B-560 devices and are not corrected.

Nuclear magnetic resonance (NMR) spectra were recorded on $300,400,600$ or $700 \mathrm{MHz}$ spectrometers. The following abbreviations and their combinations are used in the analysis of NMR spectra: $\mathrm{s}=$ singlet, $\mathrm{d}=$ doublet, $\mathrm{t}=$ triplet, $\mathrm{q}=$ quartet, $\mathrm{m}=$ multiplet, $\mathrm{br} \mathrm{s}=$ broad singlet. The ${ }^{13} \mathrm{C} \mathrm{NMR}$ spectra were recorded under broad-band proton-decoupling. NMR signals were assigned based on information from additional 2D NMR experiments (COSY, gHSQC, gHMBC, NOESY). Chemical shifts are given in ppm. Internal reference was set to the residual solvent signals $\left(\delta_{\mathrm{H}}=7.26 \mathrm{ppm}, \delta_{\mathrm{C}}=77.16 \mathrm{ppm}\right.$ for $\mathrm{CDCl}_{3}$ and $\delta_{\mathrm{H}}=2.50 \mathrm{ppm}, \delta_{\mathrm{C}}=39.52 \mathrm{ppm}$ for DMSO- $\left.d_{6}\right) .^{\mathrm{s} 2}$

Infrared (IR) spectra were recorded on a Perkin Elmer Spectrum BX-59343 instrument with a Smiths Detection DuraSamplIR II Diamond ATR sensor or a Bruker Tensor 27 FT-IR instrument with a "Platinum" Diamond ATR sensor for detection in the range $4500-600 \mathrm{~cm}^{-1}$ as a film for liquids or neat for solids.

High resolution (HRMS) mass spectra were recorded on a Finnigan MAT 90, a Finnigan MAT 95, a JEOL MStation JMS 700, a Thermo Finnigan LTQ FT Ultra Fourier Transform ion cyclotron resonance, a Q Exactive GC Orbitrap GC/MS or a Thermo Fisher Scientific LTQ Orbitrap XL. For ionization of the samples, either electron-impact ionization (EI) or electrospray ionization (ESI) was applied.

UV/Vis measurements were carried out using a J\&M TIDAS diode array spectrophotometer, which was controlled by TIDASDAQ3 (v3) software and connected to a Hellma 661.502-QX quartz Suprasil immersion probe (light path $d=5 \mathrm{~mm}$ ) via fiber optic cables and standard SMA connectors.

The reduction potentials of $p$ VQMs $1 \mathrm{1a}-\mathbf{d}$ were determined in acetonitrile on a $\mathrm{CH}$ Instruments $630 \mathrm{E}$ electrochemical analyzer using a $2 \mathrm{~mm}$ diameter platinum working electrode, a platinum wire counter electrode and an $\mathrm{Ag}$ wire pseudo-reference electrode applying a scan rate of $0.1 \mathrm{~V} / \mathrm{s}$. Cyclic voltammetry measurements were performed in deaerated acetonitrile solutions containing $0.1 \mathrm{M}$ $\mathrm{NBu}_{4} \mathrm{ClO}_{4}$ with $1\left(c \approx 3-4 \times 10^{-4} \mathrm{M}\right)$ and ferrocene $\left(c=3.8 \times 10^{-4} \mathrm{M}\right)$ as an internal standard. The $E_{1 / 2}\left(\mathrm{fc}^{+} / \mathrm{fc}\right.$ in $\mathrm{MeCN})=+0.382 \mathrm{~V}$ was used to calibrate $E_{\mathrm{p}}{ }^{\text {red }}\left(\mathbf{1}\right.$ in MeCN) vs SCE. ${ }^{\mathrm{S3}}$

For X-ray analysis, the intensity data were measured on a Bruker D8 Venture TXS system equipped with a multilayer mirror optics monochromator and a Mo Ka rotating-anode $X$-ray tube $(\lambda=0.71073$ $\AA$ A). The frames were integrated with the Bruker SAINT software package. ${ }^{S 4}$ Data were corrected for absorption effects using the Multi-Scan method (SADABS). ${ }^{S 5}$ The structures were solved and refined using the Bruker SHELXTL Software Package. ${ }^{56}$ All hydrogen atoms were calculated in positions having ideal geometry riding on their parent atoms. Xv121 was refined as a perfect inversion twin. The data have been deposited with the CCDC and can be obtained free of charge via https://www.ccdc.cam.ac.uk/structures/. 
Kinetic measurements were performed by employing stopped-flow UV/Vis photometry on Applied Photophysics SX.20 systems. The temperature $\left(20.0 \pm 0.2{ }^{\circ} \mathrm{C}\right)$ was maintained constant by using circulating bath cryostats. All solutions were prepared by using dry solvents and were kept under an atmosphere of dry nitrogen. The kinetic measurements for each $p \mathrm{VQM} /$ nucleophile combination were performed with or without added crown ether 18-crown-6 (18-c-6). 


\section{Preparation of Vinyl p-Quinone Methides 1a-d}

The vinyl $p$-quinone methides $1 \mathbf{a}-\mathbf{d}$ were synthesized following the literature procedures with only slight modifications. ${ }^{57}$

\section{(E)-2,6-Di-tert-butyl-4-(3-(4-methoxyphenyl)allylidene)cyclohexa-2,5-dien-1-one (1a)}

Crystals of 1a suitable for single crystal X-ray analysis were obtained by slow evaporation of a pentane solution as deeply red needles; $\mathrm{mp} 149-150^{\circ} \mathrm{C}$.

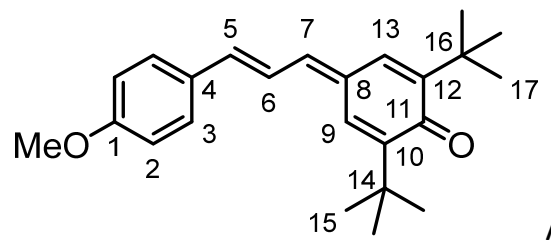

AEM39

${ }^{1} \mathrm{H}$ NMR $\left(599 \mathrm{MHz}, \mathrm{CDCl}_{3}\right): \delta=7.52-7.49(\mathrm{~m}, 3 \mathrm{H}, 3-\mathrm{H}, 9-\mathrm{H}), 7.35(\mathrm{dd}, J=15.1 \mathrm{~Hz}, 11.9 \mathrm{~Hz}, 1 \mathrm{H}, 6-\mathrm{H})$, 6.96-6.92 (m, $4 \mathrm{H}, 2-\mathrm{H}, 5-\mathrm{H}, 13-\mathrm{H}), 6.85(\mathrm{~d}, J=11.9 \mathrm{~Hz}, 1 \mathrm{H}, 7-\mathrm{H}), 3.86(\mathrm{~s}, 3 \mathrm{H}, 1-\mathrm{OMe}), 1.37(\mathrm{~s}, 9 \mathrm{H}, 15-$ H), $1.32(\mathrm{~s}, 9 \mathrm{H}, 17-\mathrm{H})$.

${ }^{13} \mathrm{C}\left\{{ }^{1} \mathrm{H}\right\}$ NMR $(101 \mathrm{MHz}, \mathrm{CDCl}): \delta=186.4\left(\mathrm{C}_{\mathrm{q}}, \mathrm{C}-11\right), 160.9\left(\mathrm{C}_{\mathrm{q}}, \mathrm{C}-1\right), 148.2\left(\mathrm{C}_{\mathrm{q}}, \mathrm{C}-10\right), 147.7\left(\mathrm{C}_{\mathrm{q}}, \mathrm{C}-12\right)$, 142.8 (CH, C-7), 141.0 (CH, C-5), 134.8 (CH, C-13), $130.4\left(\mathrm{C}_{q}, \mathrm{C}-4\right), 129.4\left(\mathrm{C}_{q}, \mathrm{C}-8\right), 129.2(\mathrm{CH}, \mathrm{C}-3), 125.6$ $(\mathrm{CH}, \mathrm{C}-9), 121.8(\mathrm{CH}, \mathrm{C}-6), 114.6(\mathrm{CH}, \mathrm{C}-2), 55.6\left(\mathrm{CH}_{3}, \mathrm{C}-18\right), 35.6\left(\mathrm{C}_{q}, \mathrm{C}-14\right), 35.2\left(\mathrm{C}_{q}, \mathrm{C}-16\right), 29.8\left(\mathrm{CH}_{3}\right.$, $\mathrm{C}-15), 29.7\left(\mathrm{CH}_{3}, \mathrm{C}-17\right)$.

HRMS (EI ${ }^{+}$): Calcd $m / z$ for $\left[\mathrm{C}_{24} \mathrm{H}_{30} \mathrm{O}_{2}{ }^{\bullet+}\right]\left[\mathrm{M}^{\bullet+}\right]: 350.2240$; Found: 350.2239.

IR (neat): 2996, 2945, 1609, 1584, 1567, 1536, 1508, 1453, 1354, 1248, 1176, 1168, 1022, 952, 929, $884,814,798 \mathrm{~cm}^{-1}$.

\section{(E)-2,6-Di-tert-butyl-4-(3-phenylallylidene)cyclohexa-2,5-dien-1-one (1b)}

Crystals of $\mathbf{1 b}$ suitable for single crystal $\mathrm{X}$-ray analysis were isolated upon slow diffusion of pentane into a THF solution of $\mathbf{1 b}$ at $-30^{\circ} \mathrm{C} ; \mathrm{mp} 190-191^{\circ} \mathrm{C}$.

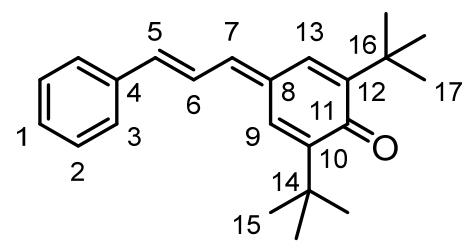

RNH110u

${ }^{1} \mathrm{H} \mathrm{NMR}\left(400 \mathrm{MHz}, \mathrm{CDCl}_{3}\right): \delta=7.57-7.54(\mathrm{~m}, 2 \mathrm{H}, 3-\mathrm{H}), 7.51-7.38(\mathrm{~m}, 4 \mathrm{H}, 2-\mathrm{H}, 6-\mathrm{H}, 9-\mathrm{H}), 7.36-7.32(\mathrm{~m}$, $1 \mathrm{H}, 1-\mathrm{H}), 6.99(\mathrm{~d}, J=15.2 \mathrm{~Hz}, 1 \mathrm{H}, 7-\mathrm{H}), 6.94(\mathrm{~d}, J=2.4 \mathrm{~Hz}, 1 \mathrm{H}, 13-\mathrm{H}), 6.86(\mathrm{~d}, J=11.8 \mathrm{~Hz}, 1 \mathrm{H}, 5-\mathrm{H})$, $1.37(\mathrm{~s}, 9 \mathrm{H}, 15-\mathrm{H}), 1.32(\mathrm{~s}, 9 \mathrm{H}, 17-\mathrm{H})$.

${ }^{13} \mathrm{C}\left\{{ }^{1} \mathrm{H}\right\}$ NMR (101 MHz, CDCl 3 ): $\delta=186.5\left(\mathrm{C}_{\mathrm{q}}, \mathrm{C}-11\right), 148.6\left(\mathrm{C}_{\mathrm{q}}, \mathrm{C}-10\right), 148.1\left(\mathrm{C}_{\mathrm{q}}, \mathrm{C}-12\right), 142.1(\mathrm{CH}, \mathrm{C}-5)$, 141.0 (CH, C-7), 136.5 ( $\left.\mathrm{C}_{\mathrm{q}}, \mathrm{C}-4\right), 134.7$ (CH, C-13), 131.4 (C, $\left.\mathrm{C}-8\right), 129.5$ (CH, C-1), 129.1 (CH, C-2), 127.6 $(\mathrm{CH}, \mathrm{C}-3), 125.5(\mathrm{CH}, \mathrm{C}-9), 123.7(\mathrm{CH}, \mathrm{C}-6), 35.7(\mathrm{C}, \mathrm{C}-14), 35.2\left(\mathrm{C}_{\mathrm{q}}, \mathrm{C}-16\right), 29.8\left(\mathrm{CH}_{3}, \mathrm{C}-15\right), 29.7\left(\mathrm{CH}_{3}\right.$, C-17).

HRMS (El+): Calcd $m / z$ for $\left[\mathrm{C}_{23} \mathrm{H}_{28} \mathrm{O}^{\bullet+}\right]\left[\mathrm{M}^{\bullet+}\right]$ : 320.2135; Found: 320.2135. 
IR (neat): $3005,2952,2916,2864,1585,1574,1538,1481,1450,1358,1282,1253,1200,1176,1085$, $1020,972,950,896,882,848,820,750,688 \mathrm{~cm}^{-1}$.

\section{(E)-2,6-Di-tert-butyl-4-(3-(4-chlorophenyl)allylidene)cyclohexa-2,5-dien-1-one (1c)}

Slow evaporation of a pentane solution of 1c yielded crystals (orange blocks) suitable for single crystal $\mathrm{X}$-ray analysis; $\mathrm{mp} 158-159^{\circ} \mathrm{C}$.

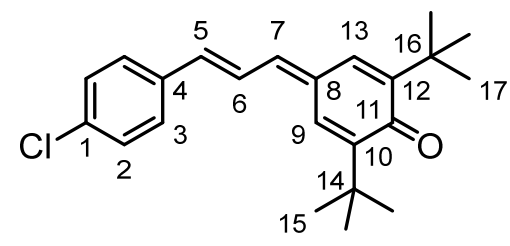

$\mathrm{RNH} 248$

${ }^{1} \mathrm{H}$ NMR $\left(599 \mathrm{MHz}, \mathrm{CDCl}_{3}\right): \delta=7.48-7.46(\mathrm{~m}, 3 \mathrm{H}, 3-\mathrm{H}, 9-\mathrm{H}), 7.42(\mathrm{dd}, J=15.2 \mathrm{~Hz}, 11.9 \mathrm{~Hz}, 1 \mathrm{H}, 6-\mathrm{H})$, 7.37-7.35 (m, $2 \mathrm{H}, 2-\mathrm{H}), 6.93-6.90(\mathrm{~m}, 2 \mathrm{H}, 13-\mathrm{H}, 5-\mathrm{H}), 6.82(\mathrm{~d}, \mathrm{~J}=11.9 \mathrm{~Hz}, 1 \mathrm{H}, 7-\mathrm{H}), 1.37(\mathrm{~s}, 9 \mathrm{H}, 15-$ H), $1.32(\mathrm{~s}, 9 \mathrm{H}, 17-\mathrm{H})$.

${ }^{13} \mathrm{C}\left\{{ }^{1} \mathrm{H}\right\}$ NMR $(101 \mathrm{MHz}, \mathrm{CDCl}): \delta=186.5\left(\mathrm{C}_{\mathrm{q}}, \mathrm{C}-11\right), 148.7\left(\mathrm{C}_{\mathrm{q}}, \mathrm{C}-10\right), 148.3\left(\mathrm{C}_{\mathrm{q}}, \mathrm{C}-12\right), 141.5(\mathrm{CH}, \mathrm{C}-7)$, $139.3(\mathrm{CH}, \mathrm{C}-5), 135.2\left(\mathrm{C}_{q}, \mathrm{C}-1\right), 135.0\left(\mathrm{C}_{q}, \mathrm{C}-4\right), 134.6(\mathrm{CH}, \mathrm{C}-13), 131.8\left(\mathrm{C}_{\mathrm{q}}, \mathrm{C}-8\right), 129.3(\mathrm{CH}, \mathrm{C}-2), 128.7$ $(\mathrm{CH}, \mathrm{C}-3), 125.4(\mathrm{CH}, \mathrm{C}-9), 124.2(\mathrm{CH}, \mathrm{C}-6), 35.7(\mathrm{C}, \mathrm{C}-14), 35.2\left(\mathrm{C}_{\mathrm{q}}, \mathrm{C}-16\right), 29.8\left(\mathrm{CH}_{3}, \mathrm{C}-15\right), 29.7\left(\mathrm{CH}_{3}\right.$, C-17).

HRMS (ESI ${ }^{+}$): Calcd $m / z$ for $\left[\mathrm{C}_{23} \mathrm{H}_{27}{ }^{35} \mathrm{ClO}^{\bullet+}\right]\left[\mathrm{M}^{\bullet+}\right]$ : 354.1745 ; Found: 354.1738.

IR (neat): 2954, 2916, 2863, 1635, 1605, 1587, 1573, 1560, 1539, 1485, 1453, 1355, 1253, 1229, 1201, $1174,1086,1021,1011,976,966,951,884,819,809,689 \mathrm{~cm}^{-1}$.

\section{(E)-2,6-Di-tert-butyl-4-(3-(4-nitrophenyl)allylidene)cyclohexa-2,5-dien-1-one (1d)}

Crystals of $1 \mathbf{d}$ suitable for single crystal X-ray analysis were isolated by slow diffusion of pentane into a THF solution of $1 \mathbf{d}$ at $-30^{\circ} \mathrm{C} ; \mathrm{mp} 192-193^{\circ} \mathrm{C}$.

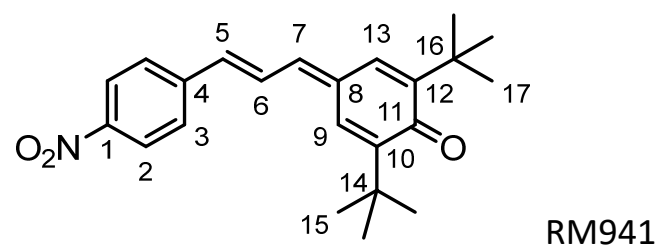

${ }^{1} \mathrm{H}$ NMR $\left(400 \mathrm{MHz}, \mathrm{CDCl}_{3}\right): \delta=8.26-8.23(\mathrm{~m}, 2 \mathrm{H}, 2-\mathrm{H}), 7.69-7.65(\mathrm{~m}, 2 \mathrm{H}, 3-\mathrm{H}), 7.58(\mathrm{dd}, J=15.2 \mathrm{~Hz}$, $11.9 \mathrm{~Hz}, 1 \mathrm{H}, 6-\mathrm{H}), 7.48(\mathrm{~d}, J=2.3 \mathrm{~Hz}, 1 \mathrm{H}, 9-\mathrm{H}), 6.99(\mathrm{~d}, J=15.3 \mathrm{~Hz}, 1 \mathrm{H}, 5-\mathrm{H}), 6.94(\mathrm{~d}, J=2.3 \mathrm{~Hz}, 1 \mathrm{H}$, 13-H), $6.84(\mathrm{~d}, J=11.9 \mathrm{~Hz}, 1 \mathrm{H}, 7-\mathrm{H}), 1.37(\mathrm{~s}, 9 \mathrm{H}, 15-\mathrm{H}), 1.32(\mathrm{~s}, 9 \mathrm{H}, 17-\mathrm{H})$.

${ }^{13} \mathrm{C}\left\{{ }^{1} \mathrm{H}\right\}$ NMR $\left(101 \mathrm{MHz}, \mathrm{CDCl}_{3}\right): \delta=186.5\left(\mathrm{C}_{\mathrm{q}}, \mathrm{C}-11\right), 149.4\left(\mathrm{C}_{\mathrm{q}}, \mathrm{C}-10\right), 149.1\left(\mathrm{C}_{\mathrm{q}}, \mathrm{C}-12\right), 147.7\left(\mathrm{C}_{\mathrm{q}}, \mathrm{C}-1\right)$, $142.7\left(\mathrm{C}_{\mathrm{q}}, \mathrm{C}-4\right), 140.0(\mathrm{CH}, \mathrm{C}-7), 137.3(\mathrm{CH}, \mathrm{C}-5), 134.4(\mathrm{CH}, \mathrm{C}-13), 133.6\left(\mathrm{C}_{\mathrm{q}}, \mathrm{C}-8\right), 127.9(\mathrm{CH}, \mathrm{C}-3), 127.6$ $(\mathrm{CH}, \mathrm{C}-6), 125.1(\mathrm{CH}, \mathrm{C}-9), 124.4(\mathrm{CH}, \mathrm{C}-2), 35.8(\mathrm{C}, \mathrm{C}-14), 35.3\left(\mathrm{C}_{\mathrm{q}}, \mathrm{C}-16\right), 29.8\left(\mathrm{CH}_{3}, \mathrm{C}-15\right), 29.7\left(\mathrm{CH}_{3}\right.$, C-17).

HRMS (EI ${ }^{+}$: Calcd $m / z$ for $\left[\mathrm{C}_{23} \mathrm{H}_{27} \mathrm{NO}_{3}{ }^{\circ+}\right]\left[\mathrm{M}^{\bullet+}\right]$ : 365.1985; Found: 365.1980 .

IR (neat): 2999, 2959, 2918, 2867, 1606, 1589, 1540, 1516, 1485, 1453, 1360, 1334, 1315, 1254, 1202, $1106,1086,1022,966,953,884,865,834,820,746,686 \mathrm{~cm}^{-1}$. 


\section{UV/Vis Spectra and Molar Absorption Coefficients of pVQMs 1a-d}

A pVQM solution in DMSO (10 to $20 \mathrm{mg}$ in $10 \mathrm{~mL}$ solvent) was added stepwise to a certain volume of DMSO (typically 20 to $25 \mathrm{~mL}$ ). Molar absorption coefficients for $p$ VQMs 1a-d were determined from the slopes of the linear correlations of the absorbance $A$ (at the absorption maximum) of the resulting DMSO solutions with the $p V Q M$ concentration.

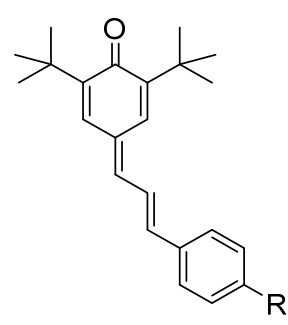

1a, $\lambda_{\max }=432 \mathrm{~nm}, \varepsilon=5.60 \times 10^{4} \mathrm{M}^{-1} \mathrm{~cm}^{-1}$

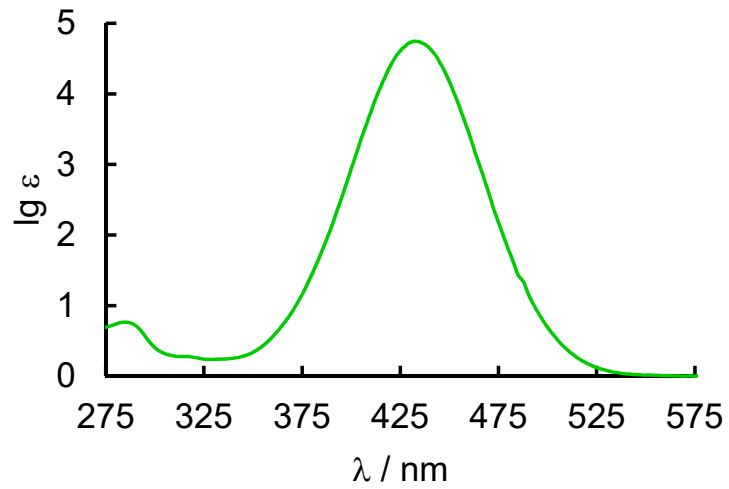

1c, $\lambda_{\max }=408 \mathrm{~nm}, \varepsilon=5.44 \times 10^{4} \mathrm{M}^{-1} \mathrm{~cm}^{-1}$

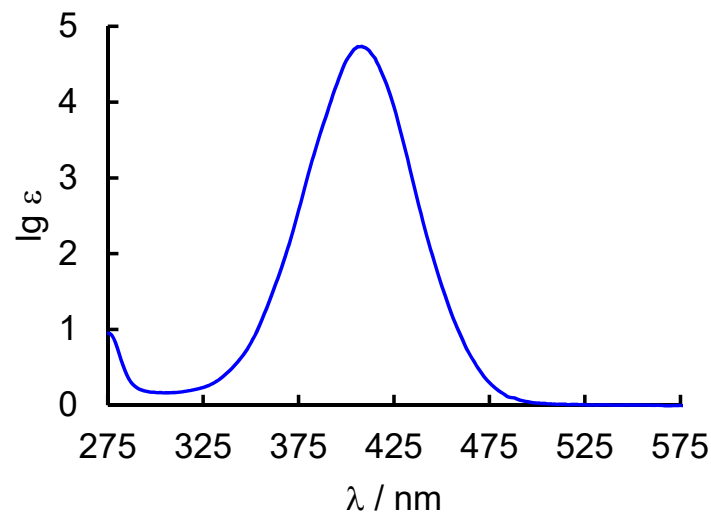

\begin{tabular}{cll}
$p \vee Q M$ & $\lambda_{\max }(\mathrm{nm})$ \\
\hline 1a & $\mathrm{OMe}$ & 432 \\
1b & $\mathrm{H}$ & 405 \\
1c & $\mathrm{Cl}$ & 408 \\
1d & $\mathrm{NO}_{2}$ & 429
\end{tabular}

1b, $\lambda_{\max }=405 \mathrm{~nm}, \varepsilon=4.76 \times 10^{4} \mathrm{M}^{-1} \mathrm{~cm}^{-1}$

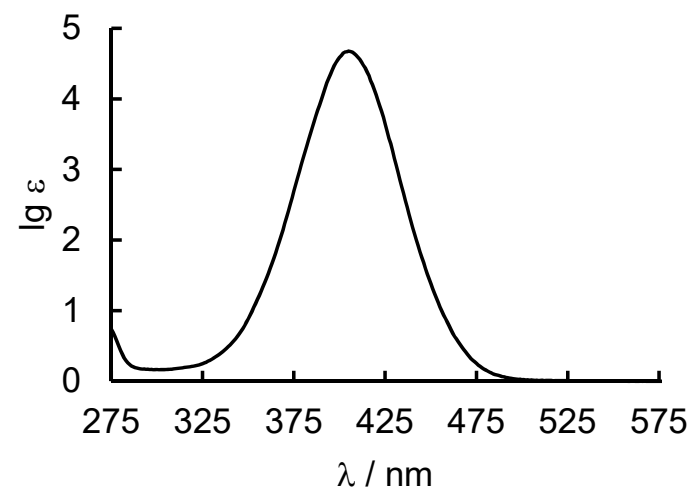

1d, $\lambda_{\max }=429 \mathrm{~nm}, \varepsilon=4.96 \times 10^{4} \mathrm{M}^{-1} \mathrm{~cm}^{-1}$

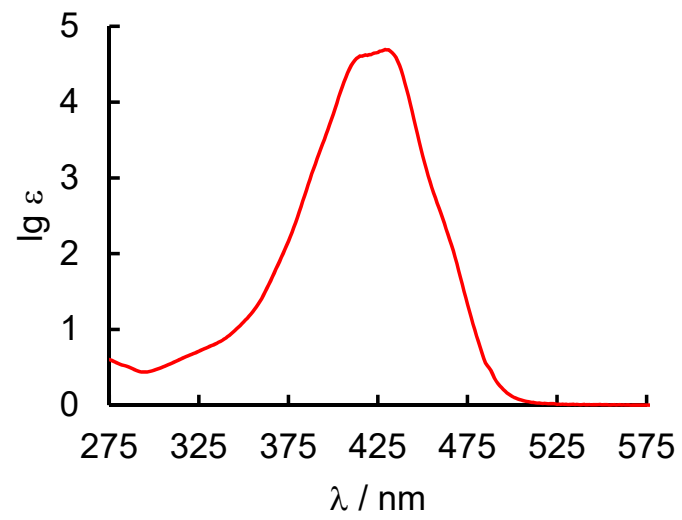




\section{Electrochemical Characterization of $p$ VQMs 1a-d}

Cyclic voltammetry measurements were performed in deaerated acetonitrile solutions containing 0.1 $\mathrm{M} \mathrm{NBu}_{4} \mathrm{ClO}_{4}$ by using a $2 \mathrm{~mm}$ diameter platinum working electrode, a platinum wire counter electrode and an $\mathrm{Ag}$ wire pseudo-reference electrode applying a scan rate of $0.1 \mathrm{~V} / \mathrm{s}$. Ferrocene was used as an internal standard for calibration. Owing to the non-reversibility of the $p V Q M$ reduction, only peak potentials $E_{p}^{\text {red }}$ were determined.

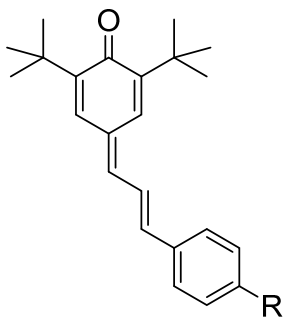

\begin{tabular}{cll}
$p V Q M$ & $\mathrm{R}$ & $E_{\mathrm{p}}{ }^{\mathrm{red}}(\mathrm{V})$ \\
\hline $\mathbf{1 a}$ & $\mathrm{OMe}$ & -1.20 \\
$\mathbf{1 b}$ & $\mathrm{H}$ & -1.14 \\
$\mathbf{1 c}$ & $\mathrm{Cl}$ & -1.11 \\
$\mathbf{1 d}$ & $\mathrm{NO}_{2}$ & $(-0.88)$
\end{tabular}
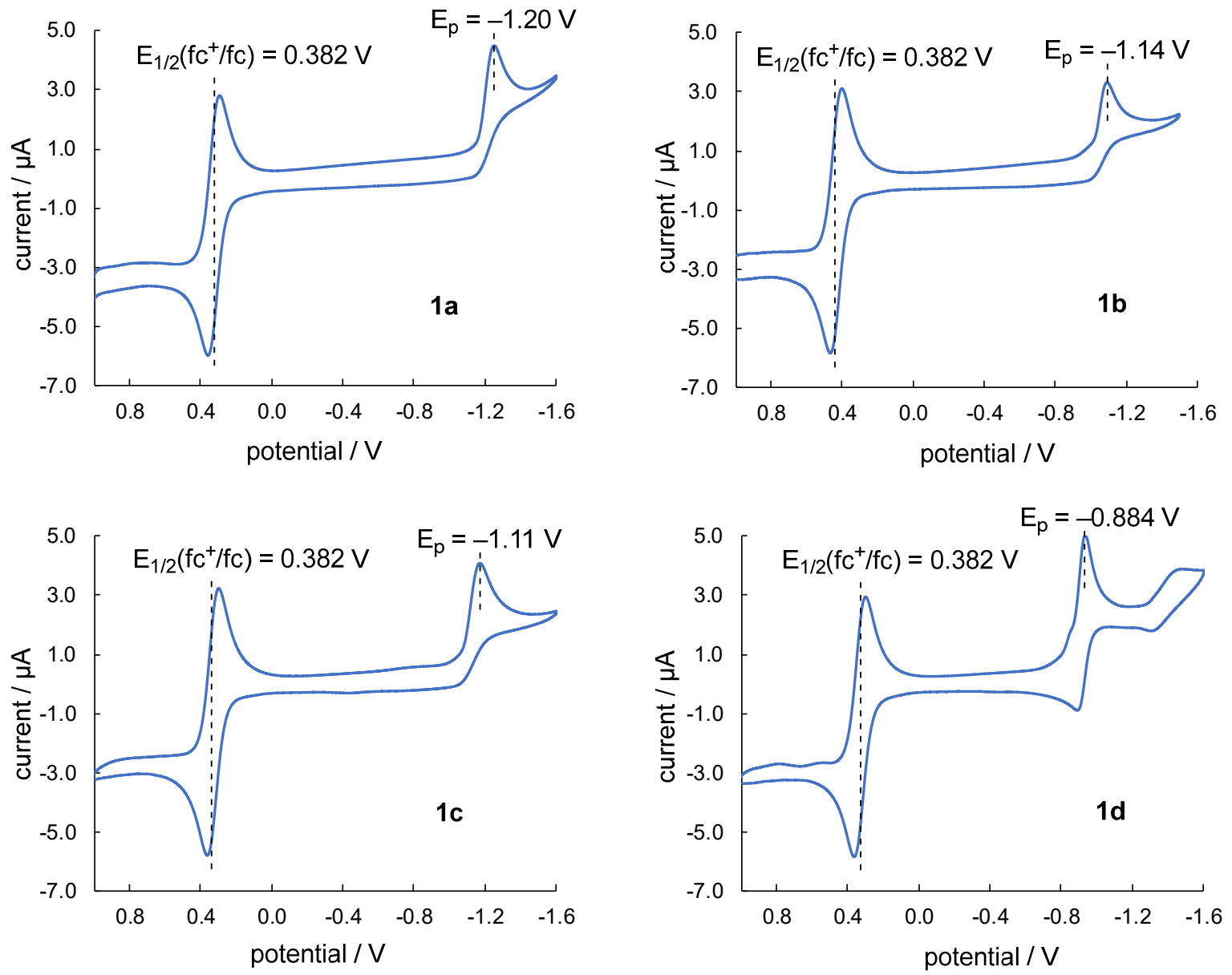


\section{Crystallographic Details of pVQMs}

Table S1. Crystallographic data for 1a (xv121), 1b (wv522), 1c (xv122), and 1d (xv123).

\begin{tabular}{|c|c|c|c|c|}
\hline & $\begin{array}{l}1 \mathbf{a} \\
(x v 121)\end{array}$ & $\begin{array}{l}\mathbf{1 b} \\
\text { (wv522) }\end{array}$ & $\begin{array}{l}\text { 1c } \\
(x v 122)\end{array}$ & $\begin{array}{l}\mathbf{1 d} \\
(x v 123)\end{array}$ \\
\hline CCDC & 1973228 & 1973229 & 1973230 & 1973231 \\
\hline net formula & $\mathrm{C}_{24} \mathrm{H}_{30} \mathrm{O}_{2}$ & $\mathrm{C}_{23} \mathrm{H}_{28} \mathrm{O}$ & $\mathrm{C}_{23} \mathrm{H}_{27} \mathrm{ClO}$ & $\mathrm{C}_{23} \mathrm{H}_{27} \mathrm{NO}_{3}$ \\
\hline$M_{\mathrm{r}} / \mathrm{g} \mathrm{mol}^{-1}$ & 350.48 & 320.45 & 354.89 & 365.45 \\
\hline crystal size/mm & $0.09 \times 0.05 \times 0.04$ & $0.09 \times 0.08 \times 0.04$ & $0.10 \times 0.03 \times 0.03$ & $0.09 \times 0.05 \times 0.03$ \\
\hline$T / \mathrm{K}$ & $112 .(2)$ & 107.(2) & $112 .(2)$ & $112 .(2)$ \\
\hline crystal system & orthorhombic & monoclinic & monoclinic & monoclinic \\
\hline space group & 'P m n 21' & 'P 1 21/n 1' & 'P 1 21/n 1' & 'P 1 21/c 1 ' \\
\hline$a / \AA$ & $28.072(3)$ & $8.7742(3)$ & $9.5906(4)$ & $13.4443(5)$ \\
\hline$b / \AA$ & $14.4136(13)$ & $17.6242(6)$ & $14.9129(7)$ & $17.5162(6)$ \\
\hline$c / \AA ̊$ & $20.5142(18)$ & $12.3375(5)$ & 14.1044(7) & $17.7087(6)$ \\
\hline$\alpha /^{\circ}$ & 90 & 90 & 90 & 90 \\
\hline$\beta /^{\circ}$ & 90 & $100.111(2)$ & $95.548(2)$ & $103.2330(10)$ \\
\hline$\gamma /{ }^{\circ}$ & 90 & 90 & 90 & 90 \\
\hline$V / \AA^{3}$ & $8300.4(13)$ & $1878.22(12)$ & $2007.81(16)$ & $4059.5(2)$ \\
\hline$Z$ & 16 & 4 & 4 & 8 \\
\hline calc. density $/ \mathrm{g} \mathrm{cm}^{-3}$ & 1.122 & 1.133 & 1.174 & 1.196 \\
\hline$\mu / \mathrm{mm}^{-1}$ & 0.069 & 0.067 & 0.198 & 0.079 \\
\hline Transm. factor range & $0.95-1.00$ & $0.96-1.00$ & $0.97-0.99$ & $0.98-1.00$ \\
\hline refls. measured & 114405 & 19955 & 35150 & 66658 \\
\hline$R_{\text {int }}$ & 0.0774 & 0.0463 & 0.0451 & 0.0568 \\
\hline mean $\sigma(I) / I$ & 0.0483 & 0.0393 & 0.0271 & 0.0319 \\
\hline$\theta$ range & $2.392-26.372$ & $3.125-27.482$ & $2.533-27.102$ & $2.325-26.021$ \\
\hline observed refls. & 13272 & 3347 & 3758 & 6250 \\
\hline$x, y$ (weighting) & $0.0513,1.2388$ & $0.0417,0.7948$ & $0.0427,0.9015$ & $0.0408,2.0780$ \\
\hline refls in refinement & 17304 & 4299 & 4430 & 7993 \\
\hline parameters & 1030 & 223 & 232 & 499 \\
\hline restraints & 1 & 0 & 0 & 0 \\
\hline$R\left(F_{\mathrm{obs}}\right)$ & 0.0450 & 0.0469 & 0.0355 & 0.0432 \\
\hline$R_{\mathrm{w}}\left(F^{2}\right)$ & 0.1203 & 0.1127 & 0.0975 & 0.1118 \\
\hline$S$ & 1.050 & 1.038 & 1.037 & 1.041 \\
\hline shift/error $r_{\max }$ & 0.001 & 0.001 & 0.001 & 0.001 \\
\hline max el. Dens./e $\AA^{-3}$ & 0.166 & 0.252 & 0.271 & 0.251 \\
\hline min el. Dens./e $\AA^{-3}$ & -0.213 & -0.214 & -0.301 & -0.229 \\
\hline
\end{tabular}

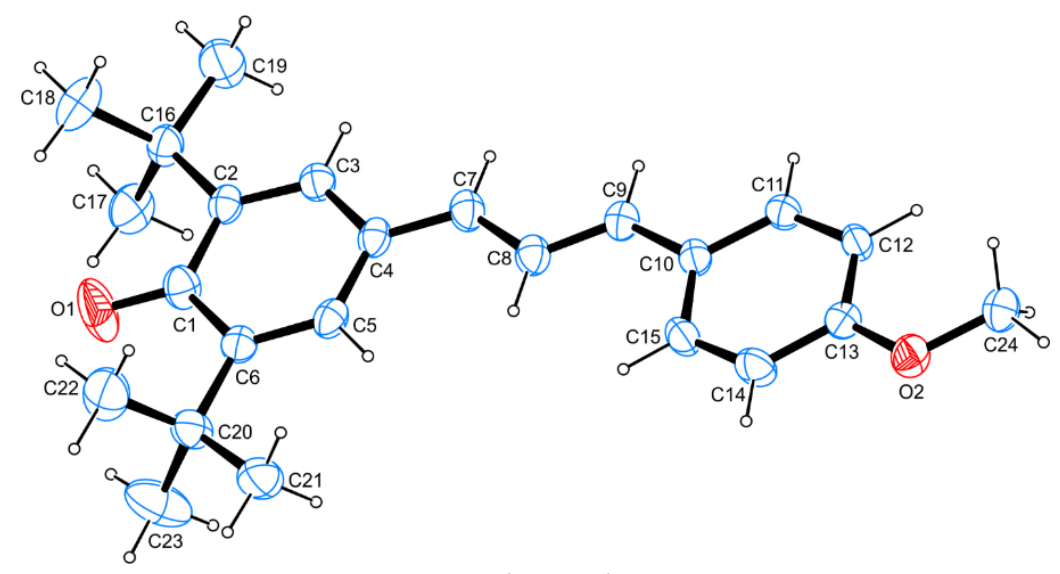

$1 a(x v 121)$

Refined as perfect inversion twin. The asymmetric unit contains four molecular units (on five sites), one of which is depicted above. 


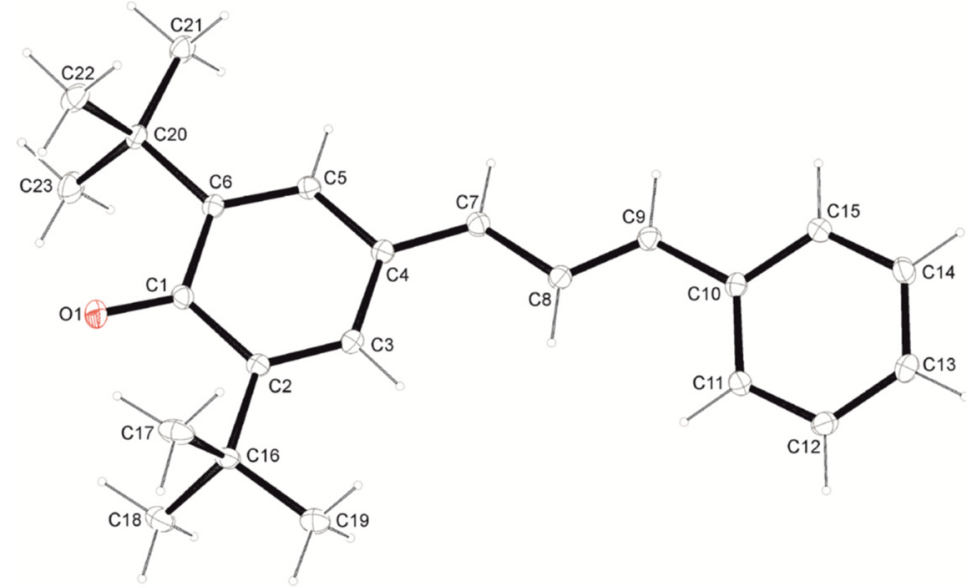

1b (wv522)

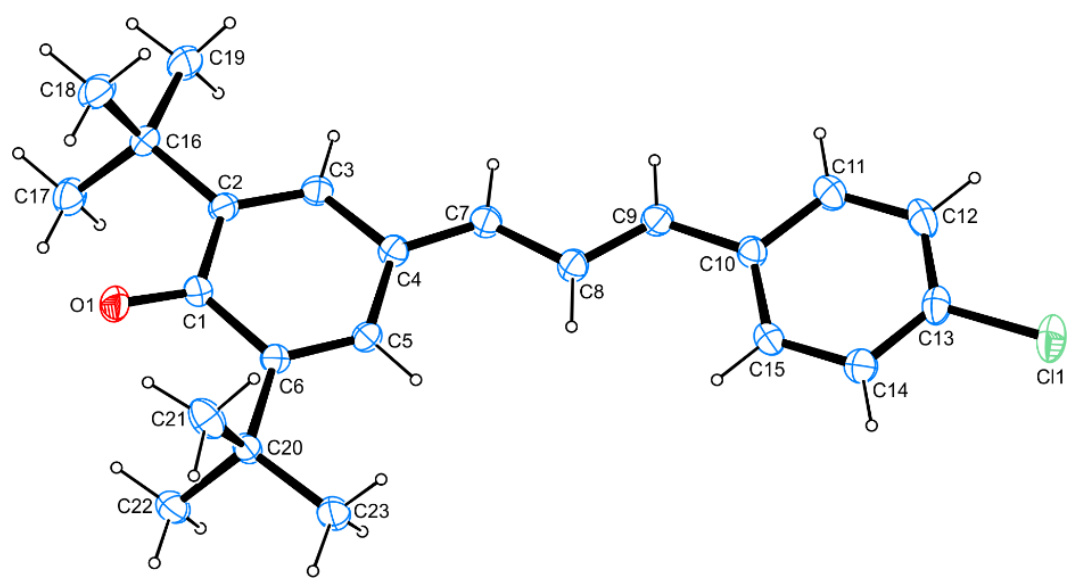

1c (xv122)

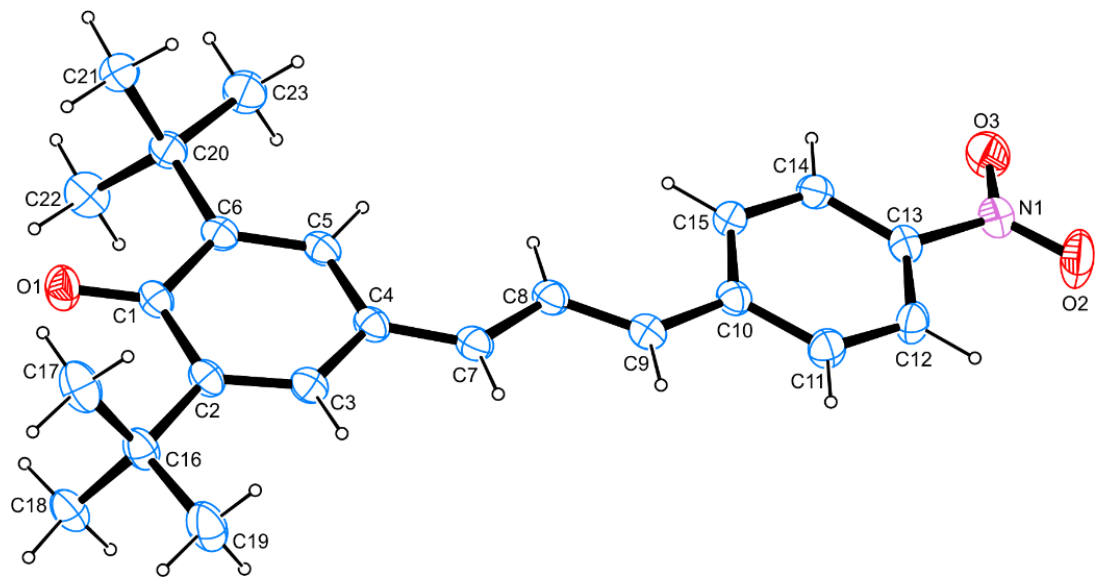

1d (xv123) 


\section{Products of the Reactions of pVQMs 1 with Nucleophiles 2}

\section{(E)-2,6-Di-tert-butyl-4-(1-(4-methoxyphenyl)-4-nitropent-1-en-3-yl)phenol (3aa) and}

(E)-2,6-Di-tert-butyl-4-(3-(4-methoxyphenyl)-4-nitropent-1-en-1-yl)phenol (4aa)

In a flame-dried Schlenk flask under nitrogen to a stirred solution of KOtBu (12.8 mg, $0.114 \mathrm{mmol}, 2$ equiv) in DMSO (1.5 mL) was added nitroethane $(2 \mathrm{a}-\mathrm{H}, 8.57 \mathrm{mg}, 0.114 \mathrm{mmol}, 2$ equiv). The clear solution was allowed to stir for one minute. Next, a solution of $1 \mathrm{a}(20.0 \mathrm{mg}, 0.0571 \mathrm{mmol}, 1$ equiv) in DMSO ( $1 \mathrm{~mL}$, dissolved with the help of heating) was added. The reaction mixture was stirred for another $30 \mathrm{~min}$ at room temperature and quenched with saturated $\mathrm{NH}_{4} \mathrm{Cl}$ solution $(30 \mathrm{~mL})$. Diethyl ether $(20 \mathrm{~mL})$ was added, the phases were separated and the aqueous phase was extracted with diethyl ether $(2 \times 20 \mathrm{~mL})$. The combined organic phases were washed with brine $(2 \times 20 \mathrm{~mL})$, dried over $\mathrm{Na}_{2} \mathrm{SO}_{4}$, filtered and concentrated under reduced pressure. The crude product was purified by column chromatography (silica gel, pentane/ethyl acetate $=92 / 8$ ) to give $20.4 \mathrm{mg}(65 \%)$ as a $60 / 40$-mixture of 1,6- and 1,8-regioisomers 3aa and 4aa, respectively.

Integrals of the ${ }^{13} \mathrm{C}$ NMR signals for $\mathrm{C}-5$ and $\mathrm{C}-7$, respectively, were used to determine the ratio of regioand diastereomers in the product mixture. Both regioisomers were formed as a mixture of diastereomers: for the 1,6-regioisomer 3aa: $d r=1.8 / 1$; for the 1,8-regioisomer 4aa: $d r=1.1 / 1$. Several resonances could be assigned to the major diastereomer of the 1,6-adduct 3aa. Integrals of the ${ }^{1} \mathrm{H}$ NMR signals for $1-\mathrm{OH}$ (4 singlets at $\delta 5.13,5.18,5.21,5.26 \mathrm{ppm})$ show a $58 / 42$ ratio of 3aa $(\mathrm{dr}=1.7 / 1)$ and $4 \mathrm{aa}(\mathrm{dr}=1.3 / 1)$.
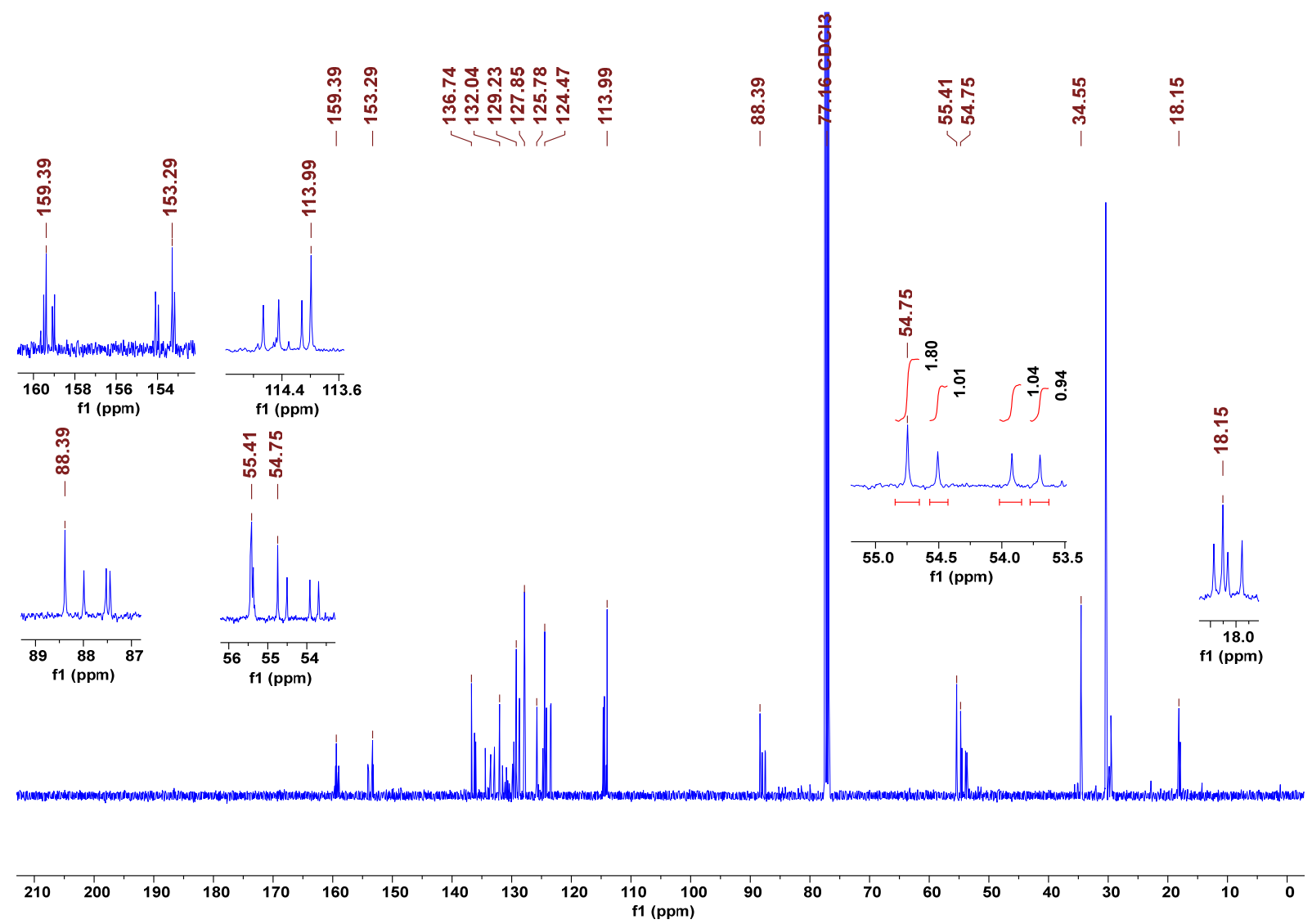

${ }^{13} \mathrm{C}$ NMR spectrum of the $3 a a / 4 a a$ mixture $\left(100.6 \mathrm{MHz}, \mathrm{CDCl}_{3}\right)$. 

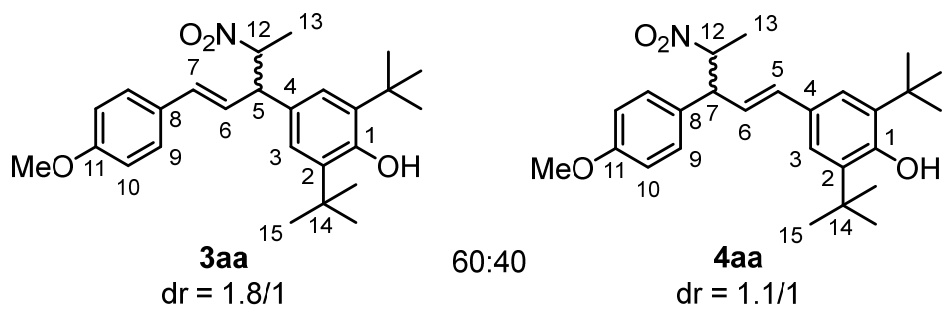

RNH266F1

${ }^{1} \mathrm{H}$ NMR $\left(400 \mathrm{MHz}, \mathrm{CDCl}_{3}\right): \delta=7.31-7.28(\mathrm{~m}, 2 \mathrm{H}, 9-\mathrm{H}), 7.28-7.23\left(\mathrm{~m}, 2 \mathrm{H}, 9-\mathrm{H}^{1,6}\right.$ major $), 7.20-7.16(\mathrm{~m}, 4$ $\mathrm{H}, 9-\mathrm{H}), 7.16(\mathrm{~s}, 2 \mathrm{H}, 3-\mathrm{H}), 7.12(\mathrm{~s}, 2 \mathrm{H}, 3-\mathrm{H}), 7.02\left(\mathrm{~s}, 2 \mathrm{H}, 3-\mathrm{H}^{1,6}\right.$ minor $), 6.99\left(\mathrm{~s}, 2 \mathrm{H}, 3-\mathrm{H}^{1,6}\right.$ major $), 6.91-6.87$ $(\mathrm{m}, 2 \mathrm{H}, 10-\mathrm{H}), 6.87-6.83(\mathrm{~m}, 4 \mathrm{H}, 10-\mathrm{H}), 6.83-6.79\left(\mathrm{~m}, 2 \mathrm{H}, 10-\mathrm{H}^{1,6}\right.$ major $), 6.50(\mathrm{~d}, J=15.6 \mathrm{~Hz}, 1 \mathrm{H}$, 5- $\left.\mathrm{H}^{1,8 \text { major }}\right), 6.49$ (d, J = 15.6 Hz, $\left.1 \mathrm{H}, 7-\mathrm{H}^{1,6 \text { minor }}\right), 6.40$ (d, $\left.J=15.6 \mathrm{~Hz}, 1 \mathrm{H}, 7-\mathrm{H}^{1,6 \text { major }}\right), 6.38$ (d, J = 15.7 $\left.\mathrm{Hz}, 1 \mathrm{H}, 5-\mathrm{H}^{1,8 \text { minor }}\right), 6.19$ (dd, J=15.6 Hz, $\left.9.0 \mathrm{~Hz}, 1 \mathrm{H}, 6-\mathrm{H}^{1,6 \text { major }}\right), 6.12$ (dd, J=15.3 Hz, $8.9 \mathrm{~Hz}, 1 \mathrm{H}, 6-\mathrm{H}$ ), $6.08(\mathrm{dd}, J=15.3 \mathrm{~Hz}, 9.2 \mathrm{~Hz}, 1 \mathrm{H}, 6-\mathrm{H}), 6.01(\mathrm{dd}, J=15.6 \mathrm{~Hz}, 9.2 \mathrm{~Hz}, 1 \mathrm{H}, 6-\mathrm{H}), 5.26(\mathrm{~s}, 1 \mathrm{H}, 1-\mathrm{OH}), 5.21$ (s, $1 \mathrm{H}, 1-\mathrm{OH}), 5.18\left(\mathrm{~s}, 1 \mathrm{H}, 1-\mathrm{OH}^{1,6}\right.$ major $), 5.13(\mathrm{~s}, 1 \mathrm{H}, 1-\mathrm{OH}), 4.96-4.81(\mathrm{~m}, 1 \mathrm{H}, 12-\mathrm{H}), 3.94-3.89(\mathrm{~m}, 1$ $\mathrm{H}, 5-\mathrm{H} / 7-\mathrm{H}), 3.80(\mathrm{~s}, 3 \mathrm{H}, 11-\mathrm{OMe}), 3.80(\mathrm{~s}, 3 \mathrm{H}, 11-\mathrm{OMe}), 3.78\left(\mathrm{~s}, 3 \mathrm{H}, 11-\mathrm{OMe}^{1,6}\right.$ major $), 3.78$ (s, $3 \mathrm{H}, 11-$ OMe), 3.75-3.70 (m, $1 \mathrm{H}, 5-\mathrm{H} / 7-\mathrm{H}), 1.66-1.61(\mathrm{~m}, 3 \mathrm{H}, 13-\mathrm{H}), 1.44\left(\mathrm{~s}, 18 \mathrm{H}, 15-\mathrm{H}^{1,6}\right.$ major $), 1.44(\mathrm{~s}, 18 \mathrm{H}$, 15-H), $1.42(\mathrm{~s}, 18 \mathrm{H}, 15-\mathrm{H}), 1.42(\mathrm{~s}, 18 \mathrm{H}, 15-\mathrm{H}), 1.40-1.37(\mathrm{~m}, 3 \mathrm{H}, 13-\mathrm{H})$.

${ }^{13} \mathrm{C}$ NMR (101 MHz, CDCl $): \delta=159.5\left(C_{q}, C-11\right), 159.4\left(C_{q}, C-11^{1,6}\right.$ major $), 159.1$ $\left(C_{q}, C-11\right), 159.0\left(C_{q}, C-11\right), 154.1\left(C_{q}, C-1\right), 154.0\left(C_{q}, C-1\right), 153.3$ ( $\left.C_{q}, C-1^{1,6 \text { major }}\right)$, $153.2\left(C_{q}, C-1\right), 136.7\left(C_{q}, C-2^{1,6}\right.$ major $), 136.3\left(C_{q}, C-2\right), 136.2\left(C_{q}, C-2\right), 136.0\left(C_{q}\right.$, C-2), $134.4(\mathrm{CH}, \mathrm{C}-5), 133.5$ (CH, C-5), 132.9 (CH, C-7), 132.0 (CH, C-7 1,6 major $)$, $131.5\left(C_{A r}\right), 131.1\left(C_{A r}\right), 130.9\left(C_{A r}\right), 130.7\left(C_{A r}\right), 130.4\left(C_{A r}\right), 130.3\left(C_{A r}\right), 129.9$ $\left(C_{\mathrm{Ar}}\right), 129.6\left(\mathrm{C}_{\mathrm{Ar}}\right), 129.6\left(\mathrm{C}_{\mathrm{Ar}}\right), 129.2\left(\mathrm{C}_{\mathrm{Ar}}\right), 128.7\left(\mathrm{C}_{\mathrm{Ar}}\right), 127.9\left(\mathrm{CH}, \mathrm{C}-9^{1,6}\right.$ major $)$, $127.8\left(\mathrm{C}_{\mathrm{Ar}}\right), 125.8\left(\mathrm{CH}, \mathrm{C}-6^{1,6}\right.$ major $), 124.8\left(\mathrm{C}_{\mathrm{Ar}}\right), 124.5\left(\mathrm{CH}, \mathrm{C}-3^{1,6}\right.$ major $), 124.4\left(\mathrm{C}_{\mathrm{Ar}}\right)$, $124.2\left(\mathrm{C}_{\mathrm{Ar}}\right), 123.5\left(\mathrm{C}_{\mathrm{Ar}}\right), 123.5\left(\mathrm{C}_{\mathrm{Ar}}\right), 123.4\left(\mathrm{C}_{\mathrm{Ar}}\right), 114.7(\mathrm{CH}, \mathrm{C}-10), 114.4(\mathrm{CH}, \mathrm{C}-$ 10), $114.1(\mathrm{CH}, \mathrm{C}-10), 114.0\left(\mathrm{CH}, \mathrm{C}-10^{1,6}\right.$ major $), 88.4\left(\mathrm{CH}, \mathrm{C}-12^{1,6}\right.$ major $), 88.0(\mathrm{CH}$, $\mathrm{C}-12), 87.5(\mathrm{CH}, \mathrm{C}-12), 87.4(\mathrm{CH}, \mathrm{C}-12), 55.45\left(\mathrm{CH}_{3}, 11-\mathrm{OMe}\right), 55.43\left(\mathrm{CH}_{3}, 11-\right.$ $\mathrm{OMe}), 55.41\left(\mathrm{CH}_{3}, 11-\mathrm{OMe}^{1,6 \text { major }}\right), 55.37\left(\mathrm{CH}_{3}, 11-\mathrm{OMe}\right), 54.7$ ( $\left.\mathrm{CH}, \mathrm{C}-5^{1,6 \text { major }}\right)$, 54.5 ( $\mathrm{CH}, \mathrm{C}-5 / \mathrm{C}-7), 53.9$ ( $\mathrm{CH}, \mathrm{C}-5 / \mathrm{C}-7), 53.7$ ( $\mathrm{CH}, \mathrm{C}-5 / \mathrm{C}-7), 34.5$ ( $\mathrm{C}_{q}, \mathrm{C}-14^{1,6}$ major), $34.5\left(\mathrm{C}_{\mathrm{q}}, \mathrm{C}-14\right), 34.4\left(\mathrm{C}_{\mathrm{q}}, \mathrm{C}-14\right), 34.4(\mathrm{C}, \mathrm{C}-14), 30.42\left(\mathrm{CH}_{3}, \mathrm{C}-15\right), 30.40\left(\mathrm{CH}_{3}, \mathrm{C}-\right.$ 15), $30.35\left(\mathrm{CH}_{3}, \mathrm{C}-15\right), 18.3\left(\mathrm{CH}_{3}, \mathrm{C}-13\right), 18.2\left(\mathrm{CH}_{3}, \mathrm{C}-13^{1,6}\right.$ major $), 18.1\left(\mathrm{CH}_{3}, \mathrm{C}-\right.$ 13), $17.9\left(\mathrm{CH}_{3}, \mathrm{C}-13\right)$.

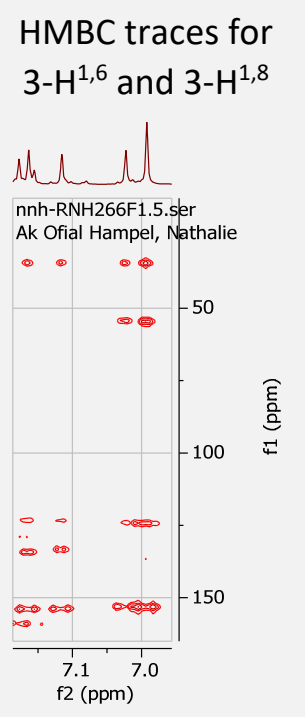

HRMS (El $\left.{ }^{+}\right)$: Calcd $m / z$ for $\left[\mathrm{C}_{26} \mathrm{H}_{35} \mathrm{NO}_{4}{ }^{\bullet+}\right]\left[\mathrm{M}^{\bullet+}\right]:$ 425.2561; Found: 425.2559 .

\section{Diethyl (E)-2-(3-(3,5-di-tert-butyl-4-hydroxyphenyl)-1-phenylallyl)malonate (4bb)}

To a stirred solution of the potassium salt $\mathbf{2 b}-\mathrm{K}(0.0574 \mathrm{mmol})$ in DMSO $(0.5 \mathrm{~mL})$ was added a solution of the $p V Q M$ 1b (1 equiv, $0.0574 \mathrm{mmol}$ ) dissolved in DMSO $(1 \mathrm{~mL}$, heating required). The reaction mixture was stirred for $30 \mathrm{~min}$ at room temperature and quenched with saturated $\mathrm{NH}_{4} \mathrm{Cl}$ solution (10 $\mathrm{mL})$. Diethyl ether $(20 \mathrm{~mL})$ was added, the phases were separated and the aqueous phase was extracted with diethyl ether $(3 \times 20 \mathrm{~mL})$. The combined organic phases were washed with water $(3 \times$ $20 \mathrm{~mL}$ ), dried over $\mathrm{Na}_{2} \mathrm{SO}_{4}$, filtered and concentrated under reduced pressure. The product $\mathbf{4 b b}$ was isolated after column chromatography (silica, pentane/ethyl acetate $=97 / 3$ ) as a yellow viscous oil (24.8 mg, 90\%). 


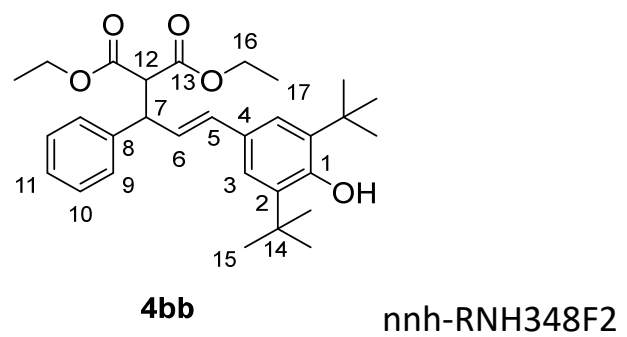

${ }^{1} \mathrm{H}$ NMR $\left(400 \mathrm{MHz}, \mathrm{CDCl}_{3}\right): \delta=7.30-7.29(\mathrm{~m}, 4 \mathrm{H}, 9-\mathrm{H}, 10-\mathrm{H}), 7.24-7.18(\mathrm{~m}, 1 \mathrm{H}, 11-\mathrm{H}), 7.11(\mathrm{~s}, 2 \mathrm{H}, 3-$ H), $6.41(\mathrm{~d}, J=15.7 \mathrm{~Hz}, 1 \mathrm{H}, 5-\mathrm{H}), 6.13(\mathrm{dd}, J=15.7 \mathrm{~Hz}, 8.4 \mathrm{~Hz}, 1 \mathrm{H}, 6-\mathrm{H}), 5.19(\mathrm{~s}, 1 \mathrm{H}, 1-\mathrm{OH}$ ), 4.24 (ddd, $J=11.1 \mathrm{~Hz}, 8.4 \mathrm{~Hz}, 1.0 \mathrm{~Hz}, 1 \mathrm{H}, 7-\mathrm{H}$ ), 4.19 (qd, J=7.1 Hz, $1.1 \mathrm{~Hz}, 2 \mathrm{H}, 16-\mathrm{H}$ ), 3.96 (qd, $J=7.1 \mathrm{~Hz}, J=1.8$ $\left.\mathrm{Hz}, 2 \mathrm{H}, 16^{\prime}-\mathrm{H}\right), 3.93(\mathrm{~d}, J=11.1 \mathrm{~Hz}, 1 \mathrm{H}, 12-\mathrm{H}), 1.41(\mathrm{~s}, 18 \mathrm{H}, 15-\mathrm{H}), 1.24(\mathrm{t}, J=7.1 \mathrm{~Hz}, 3 \mathrm{H}, 17-\mathrm{H}), 1.00$ $\left(\mathrm{t},{ }^{3} \mathrm{~J}_{\mathrm{H} 16-\mathrm{H} 17^{\prime}}=7.1 \mathrm{~Hz}, 3 \mathrm{H}, 17^{\prime}-\mathrm{H}\right)$.

${ }^{13} \mathrm{C}$ NMR (101 MHz, CDCl $): \delta=168.1\left(\mathrm{C}_{\mathrm{q}}, \mathrm{C}-13\right), 167.7\left(\mathrm{C}_{\mathrm{q}}, \mathrm{C}-13^{\prime}\right), 153.7\left(\mathrm{C}_{\mathrm{q}}, \mathrm{C}-1\right), 140.8\left(\mathrm{C}_{\mathrm{q}}, \mathrm{C}-8\right), 136.0$ $\left(\mathrm{C}_{\mathrm{q}}, \mathrm{C}-2\right), 132.4(\mathrm{CH}, \mathrm{C}-5), 128.7(\mathrm{CH}, \mathrm{C}-10), 128.3\left(\mathrm{C}_{\mathrm{q}}, \mathrm{C}-4\right), 128.2(\mathrm{CH}, \mathrm{C}-9), 127.1(\mathrm{CH}, \mathrm{C}-11), 126.5(\mathrm{CH}$, $\mathrm{C}-6), 123.3(\mathrm{CH}, \mathrm{C}-3), 61.7\left(\mathrm{CH}_{2}, \mathrm{C}-16\right), 61.5\left(\mathrm{CH}_{2}, \mathrm{C}-16^{\prime}\right), 58.0(\mathrm{CH}, \mathrm{C}-12), 49.4(\mathrm{CH}, \mathrm{C}-7), 34.4\left(\mathrm{C}_{\mathrm{q}}, \mathrm{C}-14\right)$, $30.4\left(\mathrm{CH}_{3}, \mathrm{C}-15\right), 14.3\left(\mathrm{CH}_{3}, \mathrm{C}-17\right), 13.9\left(\mathrm{CH}_{3}, \mathrm{C}-17^{\prime}\right)$.

HRMS (El+): Calcd $m / z$ for $\mathrm{C}_{30} \mathrm{H}_{40} \mathrm{O}_{5}\left[\mathrm{M}^{+}\right.$: 480.2870 ; Found: 480.2869 .

IR (neat): 3600, 2957, 1731, 1616, 1438, 1366, 1302, 1237, 1153, 1030, 962, 883, 767, $700 \mathrm{~cm}^{-1}$.

\section{Preparation of $4 \mathrm{bb}$ on $1 \mathrm{mmol}$ scale:}

EIT-1230-01A

In a flame-dried Schlenk flask under nitrogen to a stirred solution of KOtBu (225 $\mathrm{mg}, 2.00 \mathrm{mmol})$ in DMSO $(25 \mathrm{~mL})$ was added diethyl malonate $(\mathbf{2 b}-\mathrm{H}, 305 \mu \mathrm{L}, 2.00$ $\mathrm{mmol})$. The solution was allowed to stir for $10 \mathrm{~min}$. Next, a solution of the $p$ VQM 1b $(321 \mathrm{mg}, 1.00 \mathrm{mmol})$ in DMSO (10 mL, dissolved with the help of heating) was added. The reaction mixture was stirred for another $60 \mathrm{~min}$ at room temperature and quenched with saturated $\mathrm{NH}_{4} \mathrm{Cl}$ solution $(50 \mathrm{~mL})$. The reaction mixture was extracted with diethyl ether $(5 \times 50 \mathrm{~mL})$. The combined organic phases were washed with brine $(1 \times 200 \mathrm{~mL})$, dried over $\mathrm{Na}_{2} \mathrm{SO}_{4}$, filtered and concentrated under reduced pressure. The crude product was purified by column chromatography (silica gel, heptane/ethyl acetate $=10 / 1$ ) to give $\mathbf{4 b b}$ as a viscous oil (372 mg, 77\%).

${ }^{1} \mathrm{H}(300 \mathrm{MHz})$ and ${ }^{13} \mathrm{C}(75.5 \mathrm{MHz}) \mathrm{NMR}$ spectroscopic data in $\mathrm{CDCl}_{3}$ agree with those reported above (nnh-RNH348F2).

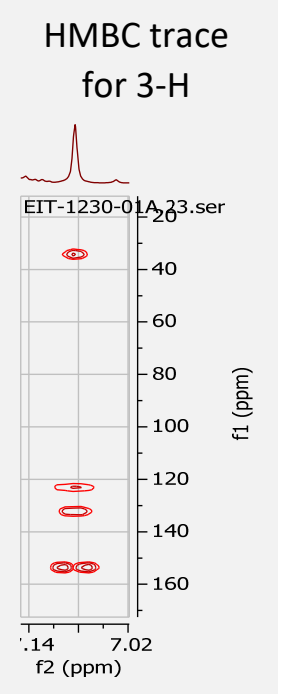

\section{In situ NMR Spectroscopic Product Studies of the Reactions of 1a-d with 2b-K}

Under an atmosphere of dry argon, a solution (or suspension) of the $p$ VQM 1 (ca. $0.015 \mathrm{mmol}$ ) in $d_{6^{-}}$ DMSO $(0.35 \mathrm{~mL})$ was added to a solution of $\mathbf{2 b}-\mathrm{K}$ (1 equiv) in $d_{6}$-DMSO $(0.35 \mathrm{~mL})$. The resulting reaction mixture was then directly analyzed by NMR spectroscopy without further workup (if the $p$ VQM was not soluble in $d_{6}$-DMSO, it dissolved after addition of the nucleophile solution).

The NMR spectroscopic investigations detected a single isomer in each investigated combination of the $p V Q M s \mathbf{1}$ with $\mathbf{2 b}-\mathrm{K}$. The HMBC experiments then provided evidence for the exclusive formation of the potassium phenolates $4-\mathrm{K}$ that originate from the $1,8-$ Michael addition of $\mathbf{2 b}-\mathrm{K}$ to the $p$ VQMs $\mathbf{1}$. 
Potassium (E)-2,6-di-tert-butyl-4-(5-ethoxy-4-(ethoxycarbonyl)-3-(4-methoxyphenyl)-5-oxopent-1en-1-yl)phenolate (4ab-K): The potassium phenolate $4 a b-K$ was obtained by mixing equimolar amounts of $1 \mathrm{a}(5.0 \mathrm{mg}, 0.014 \mathrm{mmol})$ and $2 \mathrm{~b}-\mathrm{K}(2.8 \mathrm{mg}, 0.014 \mathrm{mmol})$ in $d_{6}$-DMSO $(0.70 \mathrm{~mL})$.

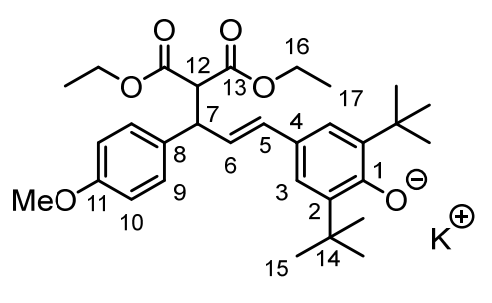

4ab-K

RNH262

${ }^{1} \mathrm{H}$ NMR (400 MHz, DMSO- $\left.d_{6}\right): \delta=7.20-7.18(\mathrm{~m}, 2 \mathrm{H}, 10-\mathrm{H}), 6.84-6.80(\mathrm{~m}, 2 \mathrm{H}, 9-$ H), $6.61(\mathrm{~s}, 2 \mathrm{H}, 3-\mathrm{H}), 6.01(\mathrm{~d}, J=15.4 \mathrm{~Hz}, 1 \mathrm{H}, 5-\mathrm{H}), 5.34(\mathrm{dd}, J=15.5 \mathrm{~Hz}, 7.1 \mathrm{~Hz}$, $1 \mathrm{H}, 6-\mathrm{H}), 4.08$ (q, J = $7.1 \mathrm{~Hz}, 2 \mathrm{H}, 16-\mathrm{H}), 3.91-3.84\left(\mathrm{~m}, 4 \mathrm{H}, 7-\mathrm{H}, 12-\mathrm{H}, 16^{\prime}-\mathrm{H}\right), 3.70$ $(\mathrm{s}, 3 \mathrm{H}, 11-\mathrm{OMe}), 1.28(\mathrm{~s}, 18 \mathrm{H}, 15-\mathrm{H}), 1.17(\mathrm{t}, J=7.1 \mathrm{~Hz}, 3 \mathrm{H}, 17-\mathrm{H}), 0.92(\mathrm{t}, J=7.1$ $\mathrm{Hz}, 3 \mathrm{H}, 17-\mathrm{H})$.

${ }^{13} \mathrm{C}$ NMR (101 MHz, DMSO- $\left.d_{6}\right): \delta=172.3\left(C_{q}, C-1\right), 167.6\left(C_{q}, C-13\right), 167.3\left(C_{q}, C-\right.$ $\left.13^{\prime}\right), 157.6\left(\mathrm{C}_{\mathrm{q}}, \mathrm{C}-11\right), 135.0\left(\mathrm{C}_{\mathrm{q}}, \mathrm{C}-2\right), 134.59\left(\mathrm{C}_{\mathrm{q}}, \mathrm{C}-8\right), 134.56(\mathrm{CH}, \mathrm{C}-5), 128.7$ (CH, C-10), $121.9(\mathrm{CH}, \mathrm{C}-3), 114.2(\mathrm{CH}, \mathrm{C}-6), 113.5(\mathrm{CH}, \mathrm{C}-9), 110.0\left(\mathrm{C}_{\mathrm{q}}, \mathrm{C}-4\right), 60.7$ $\left(\mathrm{CH}_{2}, \mathrm{C}-16\right), 60.5\left(\mathrm{CH}_{2}, \mathrm{C}-16^{\prime}\right), 57.8(\mathrm{CH}, \mathrm{C}-12), 54.9\left(\mathrm{CH}_{3}, 11-\mathrm{OMe}\right), 48.8(\mathrm{CH}, \mathrm{C}-$ 7), $34.6\left(\mathrm{C}_{\mathrm{q}}, \mathrm{C}-14\right), 29.8\left(\mathrm{CH}_{3}, \mathrm{C}-15\right), 14.0\left(\mathrm{CH}_{3}, \mathrm{C}-17\right), 13.7\left(\mathrm{CH}_{3}, \mathrm{C}-17^{\prime}\right)$.

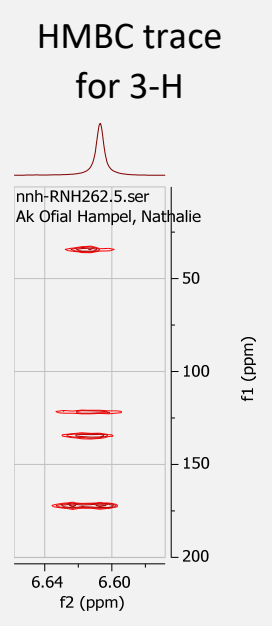

Potassium (E)-2,6-di-tert-butyl-4-(5-ethoxy-4-(ethoxycarbonyl)-5-oxo-3-phenylpent-1-en-1-

yl)phenolate (4bb-K): The potassium phenolate $4 \mathbf{b b}-K$ was obtained by mixing equimolar amounts of 1b $(5.0 \mathrm{mg}, 0.016 \mathrm{mmol})$ and $\mathbf{2 b}-\mathrm{K}(3.1 \mathrm{mg}, 0.016 \mathrm{mmol})$ in $d_{6}$-DMSO $(0.70 \mathrm{~mL})$.

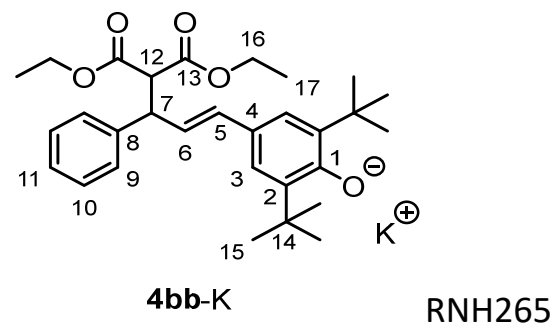

${ }^{1} \mathrm{H}$ NMR (400 MHz, DMSO- $\left.d_{6}\right): \delta=7.30-7.23(\mathrm{~m}, 4 \mathrm{H}, 9-\mathrm{H}, 10-\mathrm{H}), 7.17-7.12$ ( $\mathrm{m}, 1 \mathrm{H}, 11-\mathrm{H}$ ), $6.61(\mathrm{~s}, 2 \mathrm{H}, 3-\mathrm{H}), 6.05(\mathrm{~d}, J=15.3 \mathrm{~Hz}, 1 \mathrm{H}, 5-\mathrm{H}), 5.34$ (ddd, $J=$ $15.4 \mathrm{~Hz}, 6.0 \mathrm{~Hz}, 2.6 \mathrm{~Hz}, 1 \mathrm{H}, 6-\mathrm{H}), 4.09$ (q, J = $7.1 \mathrm{~Hz}, 2 \mathrm{H}, 16-\mathrm{H}), 3.98-3.94$ (m, $2 \mathrm{H}, 7-\mathrm{H}, 12-\mathrm{H}), 3.90-3.82\left(\mathrm{~m}, 2 \mathrm{H}, 16^{\prime}-\mathrm{H}\right), 1.28(\mathrm{~s}, 18 \mathrm{H}, 15-\mathrm{H}), 1.17(\mathrm{t}, \mathrm{J}=7.1$ $\mathrm{Hz}, 3 \mathrm{H}, 17-\mathrm{H}), 0.89\left(\mathrm{t}, J=7.1 \mathrm{~Hz}, 3 \mathrm{H}, 17^{\prime}-\mathrm{H}\right)$.

${ }^{13} \mathrm{C}$ NMR (101 MHz, DMSO): $\delta=172.3\left(C_{q}, C-1\right), 167.6\left(C_{q}, C-13\right), 167.3\left(C_{q}, C-\right.$ 13'), 142.7 ( $\left.\mathrm{C}_{q}, \mathrm{C}-8\right), 135.1$ ( $\left.\mathrm{C}_{\mathrm{q}}, \mathrm{C}-2\right), 134.9(\mathrm{CH}, \mathrm{C}-5), 128.2(\mathrm{CH}, \mathrm{C}-10), 127.6$ ( $\mathrm{CH}, \mathrm{C}-9), 126.1(\mathrm{CH}, \mathrm{C}-11), 121.9(\mathrm{CH}, \mathrm{C}-3), 113.8(\mathrm{CH}, \mathrm{C}-6), 110.0\left(\mathrm{C}_{\mathrm{q}}, \mathrm{C}-4\right)$, $60.7\left(\mathrm{CH}_{2}, \mathrm{C}-16\right), 60.5\left(\mathrm{CH}_{2}, \mathrm{C}-16^{\prime}\right), 57.5(\mathrm{CH}, \mathrm{C}-12), 49.7(\mathrm{CH}, \mathrm{C}-7), 34.6\left(\mathrm{C}_{q}, \mathrm{C}-\right.$ 14), $29.8\left(\mathrm{CH}_{3}, \mathrm{C}-15\right), 14.5\left(\mathrm{CH}_{3}, \mathrm{C}-17\right), 13.6\left(\mathrm{CH}_{3}, \mathrm{C}-17^{\prime}\right)$.

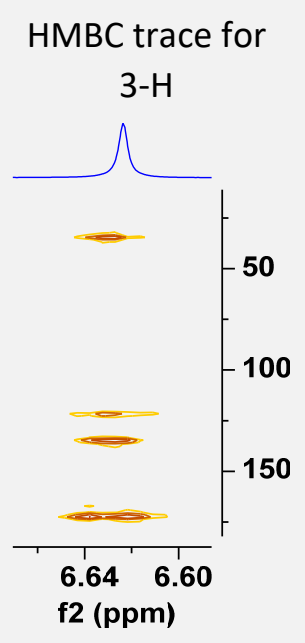


Potassium (E)-2,6-di-tert-butyl-4-(3-(4-chlorophenyl)-5-ethoxy-4-(ethoxycarbonyl)-5-oxopent-1-en1-yl)phenolate (4cb-K): The potassium phenolate $4 \mathrm{cb}-\mathrm{K}$ was obtained by mixing equimolar amounts of $1 \mathrm{c}(5.0 \mathrm{mg}, 0.014 \mathrm{mmol})$ and $2 \mathrm{~b}-\mathrm{K}(2.8 \mathrm{mg}, 0.014 \mathrm{mmol})$ in $d_{6}$-DMSO $(0.70 \mathrm{~mL})$.

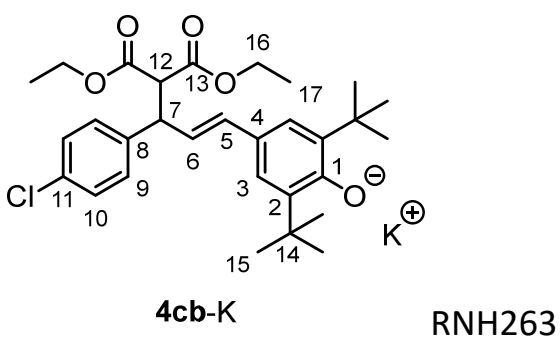

${ }^{1} \mathrm{H}$ NMR $\left(400 \mathrm{MHz}\right.$, DMSO- $\left.d_{6}\right): \delta=7.35-7.29(\mathrm{~m}, 4 \mathrm{H}, 9-\mathrm{H}, 10-\mathrm{H}), 6.62(\mathrm{~s}, 2 \mathrm{H}, 3-$ H), 6.06 (d, $J=15.3 \mathrm{~Hz}, 1 \mathrm{H}, 5-\mathrm{H}$ ), $5.32(\mathrm{dd}, J=15.3 \mathrm{~Hz}, 7.8 \mathrm{~Hz}, 1 \mathrm{H}, 6-\mathrm{H}), 4.09$ (q, $J=7.1 \mathrm{~Hz}, 2 \mathrm{H}, 16-\mathrm{H}), 4.01-3.85\left(\mathrm{~m}, 4 \mathrm{H}, 7-\mathrm{H}, 12-\mathrm{H}, 16^{\prime}-\mathrm{H}\right), 1.28(\mathrm{~s}, 18 \mathrm{H}, 15-\mathrm{H})$, $1.16(\mathrm{t}, J=7.1 \mathrm{~Hz}, 3 \mathrm{H}, 17-\mathrm{H}), 0.93\left(\mathrm{t}, J=7.1 \mathrm{~Hz}, 3 \mathrm{H}, 17^{\prime}-\mathrm{H}\right)$.

${ }^{13} \mathrm{C}$ NMR $\left(101 \mathrm{MHz}\right.$, DMSO- $\left.d_{6}\right): \delta=172.2\left(\mathrm{C}_{\mathrm{q}}, \mathrm{C}-1\right), 167.4\left(\mathrm{C}_{\mathrm{q}}, \mathrm{C}-13\right), 167.2\left(\mathrm{C}_{\mathrm{q}}, \mathrm{C}-\right.$ 13'), 141.9 ( $\left.\mathrm{C}_{q}, \mathrm{C}-8\right), 135.23\left(\mathrm{C}_{\mathrm{q}}, \mathrm{C}-2\right), 135.16$ (CH, C-5), 130.6 (C $\left.\mathrm{C}_{\mathrm{q}}, \mathrm{C}-11\right), 129.5$ ( $\mathrm{CH}, \mathrm{C}-10), 128.1(\mathrm{CH}, \mathrm{C}-9), 122.0(\mathrm{CH}, \mathrm{C}-3), 113.4(\mathrm{CH}, \mathrm{C}-6), 110.1\left(\mathrm{C}_{\mathrm{q}}, \mathrm{C}-4\right), 60.8$ $\left(\mathrm{CH}_{2}, \mathrm{C}-16\right), 60.6\left(\mathrm{CH}_{2}, \mathrm{C}-16^{\prime}\right), 57.2(\mathrm{CH}, \mathrm{C}-12), 49.0(\mathrm{CH}, \mathrm{C}-7), 34.6\left(\mathrm{C}_{\mathrm{q}}, \mathrm{C}-14\right), 29.8$ $\left(\mathrm{CH}_{3}, \mathrm{C}-15\right), 14.0\left(\mathrm{CH}_{3}, \mathrm{C}-17\right), 13.6\left(\mathrm{CH}_{3}, \mathrm{C}-17^{\prime}\right)$.

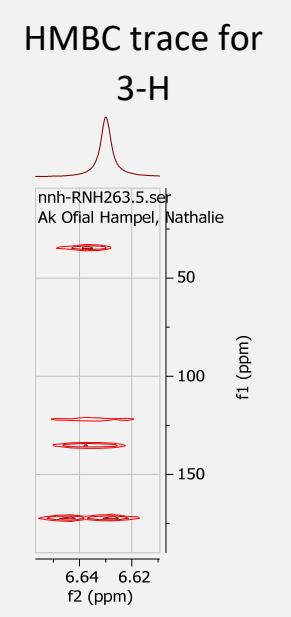

Potassium (E)-2,6-di-tert-butyl-4-(5-ethoxy-4-(ethoxycarbonyl)-3-(4-nitrophenyl)-5-oxopent-1-en-1yl)phenolate ( $4 \mathrm{db}-\mathrm{K})$ : The potassium phenolate $4 \mathrm{db}-K$ was obtained by mixing equimolar amounts of $1 \mathbf{d}(4.3 \mathrm{mg}, 0.012 \mathrm{mmol})$ and $\mathbf{2 b}-\mathrm{K}(2.3 \mathrm{mg}, 0.012 \mathrm{mmol})$ in $d_{6}$-DMSO $(0.70 \mathrm{~mL})$.

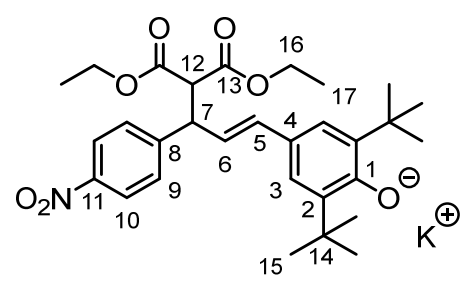

$4 \mathrm{db}-\mathrm{K}$

RNH345

$\mathrm{HMBC}$ trace for

${ }^{1} \mathrm{H}$ NMR $\left(400 \mathrm{MHz}\right.$, DMSO- $\left.d_{6}\right): \delta=8.14-8.10(\mathrm{~m}, 2 \mathrm{H}, 10-\mathrm{H}), 7.64-7.61(\mathrm{~m}, 2 \mathrm{H}$, 9-H), $6.62(\mathrm{~s}, 2 \mathrm{H}, 3-\mathrm{H}), 6.13(\mathrm{~d}, J=15.3 \mathrm{~Hz}, 1 \mathrm{H}, 5-\mathrm{H}), 5.29$ (dd, $J=15.3 \mathrm{~Hz}, 8.6$ $\mathrm{Hz}, 1 \mathrm{H}, 6-\mathrm{H}), 4.19-3.85(\mathrm{~m}, 6 \mathrm{H}, 7-\mathrm{H}, 12-\mathrm{H}, 16-\mathrm{H}), 1.27(\mathrm{~s}, 18 \mathrm{H}, 15-\mathrm{H}), 1.17(\mathrm{t}, J$ $=7.1 \mathrm{~Hz}, 3 \mathrm{H}, 17-\mathrm{H}), 0.93\left(\mathrm{t}, J=7.1 \mathrm{~Hz}, 3 \mathrm{H}, 17^{\prime}-\mathrm{H}\right)$.

${ }^{13} \mathrm{C}$ NMR (101 MHz, DMSO- $\left.d_{6}\right): \delta=172.7\left(\mathrm{C}_{\mathrm{q}}, \mathrm{C}-1\right.$, detected in the HMBC spectrum), $167.4\left(C_{q}, C-13\right), 167.2\left(C_{q}, C-13^{\prime}\right), 151.2\left(C_{q}, C-11\right), 145.7\left(C_{q}, C-8\right)$, $136.1(\mathrm{CH}, \mathrm{C}-5), 135.2\left(\mathrm{C}_{\mathrm{q}}, \mathrm{C}-2\right), 128.8(\mathrm{CH}, \mathrm{C}-9), 123.5(\mathrm{CH}, \mathrm{C}-10), 122.1(\mathrm{CH}, \mathrm{C}-$ 3), $112.0(\mathrm{CH}, \mathrm{C}-6), 109.8\left(\mathrm{C}_{\mathrm{q}}, \mathrm{C}-4\right), 60.9\left(\mathrm{CH}_{2}, \mathrm{C}-16\right), 60.8\left(\mathrm{CH}_{2}, \mathrm{C}-16^{\prime}\right), 56.7(\mathrm{CH}$, $\mathrm{C}-12), 49.5(\mathrm{CH}, \mathrm{C}-7), 34.6(\mathrm{C}, \mathrm{C}-14), 29.8\left(\mathrm{CH}_{3}, \mathrm{C}-15\right), 14.0\left(\mathrm{CH}_{3}, \mathrm{C}-17\right), 13.6\left(\mathrm{CH}_{3}\right.$, C-17').

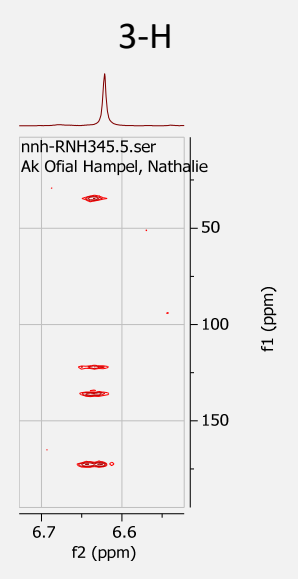




\section{Ethyl (E)-2-cyano-3-(3,5-di-tert-butyl-4-hydroxyphenyl)-5-phenylpent-4-enoate (3bc)}

A solution of the potassium salt $\mathbf{2 c}-\mathrm{K}$ was prepared by adding ethyl cyanoacetate $(21 \mu \mathrm{L}, 0.20 \mathrm{mmol}$ ) to a KOtBu (22.5 mg, $0.20 \mathrm{mmol})$ solution in DMSO $(3 \mathrm{~mL})$. After $10 \mathrm{~min}$ of stirring, the $p$ VQM $\mathbf{1 b}$ (32.1 $\mathrm{mg}, 0.10 \mathrm{mmol}$ dissolved in $1 \mathrm{~mL}$ of DMSO) was added. The reaction mixture was stirred for another $60 \mathrm{~min}$ at ambient temperature and then quenched with saturated $\mathrm{NH}_{4} \mathrm{Cl}$ solution $(30 \mathrm{~mL})$. Diethyl ether $(20 \mathrm{~mL})$ was added and the phases were separated. The aqueous phase was extracted with diethyl ether $(3 \times 20 \mathrm{~mL})$. The combined organic phases were washed with brine $(1 \times 20 \mathrm{~mL})$, dried over $\mathrm{Na}_{2} \mathrm{SO}_{4}$, filtered, and concentrated under reduced pressure. The crude product was purified by column chromatography (dichloromethane/heptane $=2 / 1$ ) to yield 3 bc.

Before workup, a 77/23 mixture of the 1,6- and the 1,8-adducts was obtained (both regioisomers as a mixture of diastereomers as indicated in the NMR spectra of the crude product mixture). After workup and column chromatography, the diastereomeric mixture of the 1,6-adduct $3 \mathrm{bc}$ was isolated $(32.5 \mathrm{mg}$, $75 \%, d r=1: 1)$, which contained only trace amounts of $4 \mathbf{b c}$.

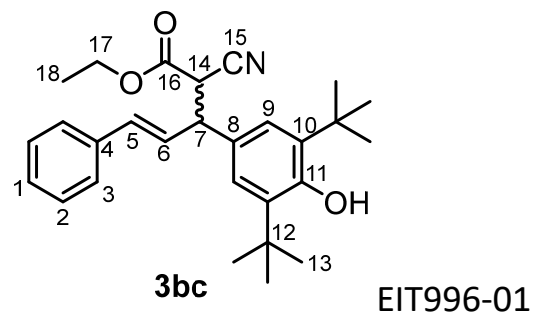

$\mathrm{R}_{\mathrm{f}}($ heptanes/ethyl acetate $=5 / 1)=0.40$

${ }^{1} \mathrm{H}$ NMR $\left(300 \mathrm{MHz}, \mathrm{CDCl}_{3}\right): \delta=7.34-7.16(\mathrm{~m}, 5 \mathrm{H}, 1-\mathrm{H}, 2-\mathrm{H}, 3-\mathrm{H}), 7.08(\mathrm{~s}, 2 \mathrm{H}, 9-$ H), 6.63-6.41 (m, $2 \mathrm{H}, 5-\mathrm{H}, 6-\mathrm{H}), 5.13(\mathrm{~s}, 1 \mathrm{H}, 11-\mathrm{OH}), 4.20-4.09(\mathrm{~m}, 3 \mathrm{H}, 7-\mathrm{H}, 17-$ H), $3.86(\mathrm{~d}, J=12.9 \mathrm{~Hz}, 14-\mathrm{H})$ and $3.84\left(\mathrm{~d}, J=11.3 \mathrm{~Hz}, 1 \mathrm{H}, 14-\mathrm{H}^{*}\right), 1.45(\mathrm{~s}, 18 \mathrm{H}$, $13-\mathrm{H}), 1.18-1.11(\mathrm{~m}, 3 \mathrm{H}, 18-\mathrm{H})$.

${ }^{13} \mathrm{C}$ NMR (75.5 MHz, CDCl 3 ): $\delta=165.3 / 165.3\left(\mathrm{C}_{q}, \mathrm{C}-16\right), 153.7 / 153.6\left(\mathrm{C}_{\mathrm{q}}, \mathrm{C}-11\right)$, 136.6/136.5/136.4 ( $\mathrm{C}_{\mathrm{q}}, \mathrm{C}-10$ and $\left.\mathrm{C}-4\right), 133.7 / 132.7(\mathrm{CH}, \mathrm{C}-5), 129.5 / 128.66\left(\mathrm{C}_{\mathrm{q}}\right.$, C-8), $128.74 / 128.71(\mathrm{CH}, \mathrm{C}-2), 128.1 / 128.0(\mathrm{CH}, \mathrm{C}-1), 127.8 / 126.5(\mathrm{CH}, \mathrm{C}-6)$, $126.8 / 126.7$ (CH, C-3), 124.6/124.4 ( $\mathrm{C}, \mathrm{C}-9), 115.9 / 115.8$ (Cq, C-15), 62.9/62.8 $\left(\mathrm{CH}_{2}, \mathrm{C}-17\right), 49.8 / 49.7(\mathrm{CH}, \mathrm{C}-7), 45.5 / 45.2(\mathrm{CH}, \mathrm{C}-14), 34.6 / 34.6\left(\mathrm{C}_{\mathrm{q}}, \mathrm{C}-12\right), 30.4$ $\left(\mathrm{CH}_{3}, \mathrm{C}-13\right), 14.10 / 14.06\left(\mathrm{CH}_{3}, \mathrm{C}-18\right)$.

HMBC trace for 9-H $\Omega$ EIT-996-01.419.ser $-20$ - -40 co

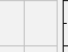
$-100$ c) -120 - 140 $\infty$ $-180$ 7.147 .07 f2 (ppm)

HRMS (ESI): Calcd $m / z$ for $\mathrm{C}_{28} \mathrm{H}_{39} \mathrm{~N}_{2} \mathrm{O}_{3}{ }^{+}\left[\mathrm{M}+\mathrm{NH}_{4}^{+}\right]$: 451.2955; Found: 451.2952.

IR (Iq. film): 3727, 3629, 2957, 2872, 2361, 2341, 1741, 1435, 1391, 1365, 1238, 1155, 1120, 1027, 965, $910,887,732,693,669,649,489 \mathrm{~cm}^{-1}$.

\section{General Procedure for the Reactions of the pVQMs 1 with 2d-K (GP 1)}<smiles>[R]C=CC=c1cc(C(C)(C)C)c(=O)c(CC(C)C)cc1=CC(C)CO[Si](C)(C)C</smiles><smiles>[R]C=CC(c1cc(C(C)(C)C)c(O)c(C(C)(C)C)c1)C(C#N)C#N</smiles><smiles>[R]C(/C=C/c1cc(CC(C)(C)C)c(O)c([Z18](C)(C)C)c1)C(C#N)C#N</smiles> 
To a stirred solution of the potassium salt $2 \mathrm{~d}-\mathrm{K}$ (2 equiv, ca. $0.1 \mathrm{mmol}$ ) in DMSO ( $1 \mathrm{~mL}$ ) was added a solution of the $p$ VQM 1 (1 equiv, ca. $0.05 \mathrm{mmol}$ ) dissolved in DMSO $(1 \mathrm{~mL})$. The reaction mixture was stirred for another 10 to $30 \mathrm{~min}$ at room temperature and then quenched with saturated $\mathrm{NH}_{4} \mathrm{Cl}$ solution $(10 \mathrm{~mL})$. Diethyl ether $(20 \mathrm{~mL})$ was added and the phases were separated. The aqueous phase was extracted with diethyl ether $(3 \times 20 \mathrm{~mL})$. The combined organic phases were washed with water $(3 \times 20 \mathrm{~mL})$, dried over $\mathrm{Na}_{2} \mathrm{SO}_{4}$, filtered, and concentrated under reduced pressure. The crude products were purified by column chromatography to give the corresponding products as yellow to orange solids.

(E)-2-(1-(3,5-Di-tert-butyl-4-hydroxyphenyl)-3-(4-methoxyphenyl)allyl)malononitrile (3ad) and (E)-2-(3-(3,5-Di-tert-butyl-4-hydroxyphenyl)-1-(4-methoxyphenyl)allyl)malononitrile (4ad)

According to GP 1: Starting from 1a $(16.8 \mathrm{mg}, 0.0479 \mathrm{mmol})$ and $2 \mathrm{~d}-\mathrm{K}(10.0 \mathrm{mg}, 0.0960 \mathrm{mmol})$ a mixture of regioisomers was isolated after column chromatography (silica, pentane/ethyl acetate $=92 / 8$ ) as a yellow oil (14.7 mg, 74\%). The 1,6- and 1,8-regioisomers 3ad and 4ad, respectively, formed in a 40/60 ratio ( ${ }^{1} \mathrm{H}$ NMR spectrum of the crude product mixture).
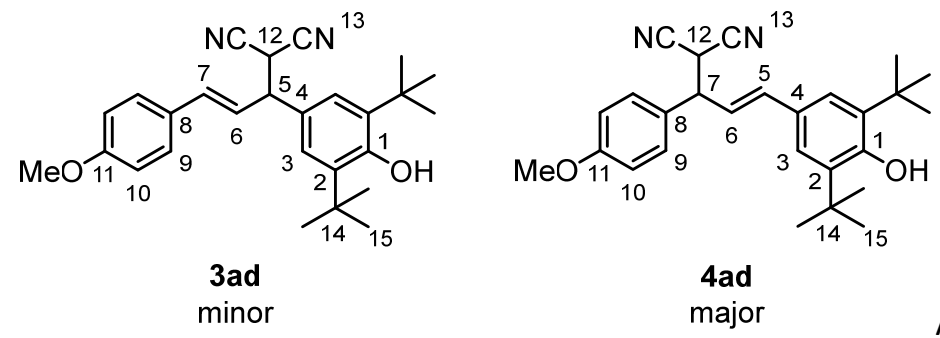

${ }^{1} \mathrm{H}$ NMR $\left(400 \mathrm{MHz}, \mathrm{CDCl}_{3}\right): \delta=7.38-7.34\left(\mathrm{~m}, 2 \mathrm{H}, 10-\mathrm{H}^{1,6}\right), 7.34-7.30(\mathrm{~m}, 2 \mathrm{H}$, $\left.10-\mathrm{H}^{1,8}\right), 7.21\left(\mathrm{~s}, 2 \mathrm{H}, 3-\mathrm{H}^{1,8}\right), 7.17\left(\mathrm{~s}, 2 \mathrm{H}, 3-\mathrm{H}^{1,6}\right), 6.97-6.93\left(\mathrm{~m}, 2 \mathrm{H}, 9-\mathrm{H}^{1,8}\right), 6.90-$ $6.86\left(\mathrm{~m}, 2 \mathrm{H}, 9-\mathrm{H}^{1,6}\right), 6.66\left(\mathrm{~d}, J=15.7 \mathrm{~Hz}, 1 \mathrm{H}, 7-\mathrm{H}^{1,6}\right), 6.62(\mathrm{~d}, J=15.7 \mathrm{~Hz}, 1 \mathrm{H}, 5-$ $\left.\mathrm{H}^{1,8}\right), 6.38-6.30\left(\mathrm{~m}, 1 \mathrm{H}, 6-\mathrm{H}^{1,6}\right), 6.24\left(\mathrm{dd}, J=15.7 \mathrm{~Hz}, 7.5 \mathrm{~Hz}, 1 \mathrm{H}, 6-\mathrm{H}^{1,8}\right), 5.32(\mathrm{~s}$, $\left.1 \mathrm{H}, 1-\mathrm{OH}^{1,8}\right), 5.30\left(\mathrm{~s}, 1 \mathrm{H}, 1-\mathrm{OH}^{1,6}\right), 4.08-3.99\left(\mathrm{~m}, 4 \mathrm{H}, 5-\mathrm{H}^{1,6}, 12-\mathrm{H}^{1,6}, 7-\mathrm{H}^{1,8}, 12-\right.$ $\left.\mathrm{H}^{1,8}\right), 3.82\left(\mathrm{~s}, 3 \mathrm{H}, 16-\mathrm{H}^{1,8}\right), 3.82\left(\mathrm{~s}, 3 \mathrm{H}, 16-\mathrm{H}^{1,6}\right), 1.45\left(\mathrm{~s}, 18 \mathrm{H}, 15-\mathrm{H}^{1,6}\right), 1.45(\mathrm{~s}$, $\left.18 \mathrm{H}, 15-\mathrm{H}^{1,8}\right)$.

${ }^{13} \mathrm{C}$ NMR $\left(101 \mathrm{MHz}, \mathrm{CDCl}_{3}\right): \delta=160.01\left(\mathrm{C}_{\mathrm{q}}, \mathrm{C}-11^{1,6}\right), 159.99\left(\mathrm{C}_{\mathrm{q}}, \mathrm{C}-11^{1,8}\right), 154.6$ $\left(C_{q}, C-1^{1,8}\right), 154.3\left(C_{q}, C-1^{1,6}\right), 136.9\left(C_{q}, C-2^{1,6}\right), 136.3\left(C H, C-5^{1,8}\right), 136.2\left(C_{q}, C-\right.$ $\left.2^{1,8}\right), 134.8\left(\mathrm{CH}, \mathrm{C}-7^{1,6}\right), 129.1\left(\mathrm{CH}, \mathrm{C}-10^{1,8}\right), 129.0\left(\mathrm{C}_{q}, \mathrm{C}-8^{1,8}\right), 128.7\left(\mathrm{C}_{\mathrm{q}}, \mathrm{C}-8^{1,6}\right)$, $128.2\left(\mathrm{CH}, \mathrm{C}-10^{1,6}\right), 127.7\left(\mathrm{C}_{\mathrm{q}}, \mathrm{C}-4^{1,6}\right), 126.9\left(\mathrm{C}_{\mathrm{q}}, \mathrm{C}-4^{1,8}\right), 124.5\left(\mathrm{CH}, \mathrm{C}-3^{1,6}\right), 123.8$ $\left(\mathrm{CH}, \mathrm{C}-3^{1,8}\right), 122.3\left(\mathrm{CH}, \mathrm{C}-6^{1,6}\right), 121.1\left(\mathrm{CH}, \mathrm{C}-6^{1,8}\right), 114.9\left(\mathrm{CH}, \mathrm{C}-9^{1,8}\right), 114.3(\mathrm{CH}, \mathrm{C}-$ $\left.9^{1,6}\right), 112.2\left(C_{q}, C-13^{1,6}\right), 112.1\left(C_{q}, C-13^{1,6}\right), 112.03\left(C_{q}, C-13^{1,8}\right), 112.01\left(C_{q}, C-\right.$

$\mathrm{HMBC}$ traces for $3-\mathrm{H}^{1,8}$ and $3-\mathrm{H}^{1,6}$

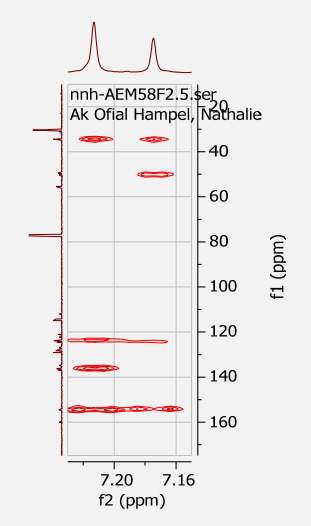
$\left.13^{1,8}\right), 55.49\left(\mathrm{CH}_{3}, 11-\mathrm{OMe}^{1,8}\right), 55.48\left(\mathrm{CH}_{3}, 11-\mathrm{OMe}^{1,6}\right), 50.3\left(\mathrm{CH}, \mathrm{C}-5^{1,6}\right), 49.5\left(\mathrm{CH}, \mathrm{C}-7^{1,8}\right), 34.6\left(\mathrm{C}_{q}, \mathrm{C}-\right.$ $\left.14^{1,6}\right)$, $34.5\left(\mathrm{C}_{q}, \mathrm{C}-14^{1,8}\right), 31.1\left(\mathrm{CH}, \mathrm{C}-12^{1,6}\right), 30.7\left(\mathrm{CH}, \mathrm{C}-12^{1,8}\right), 30.35\left(\mathrm{CH}_{3}, \mathrm{C}-15^{1,8}\right), 30.33\left(\mathrm{CH}_{3}, \mathrm{C}-15^{1,6}\right)$.

HRMS $\left(\mathrm{EI}^{+}\right)$: Calcd $m / z$ for $\left[\mathrm{C}_{27} \mathrm{H}_{30} \mathrm{~N}_{2} \mathrm{O}_{2}{ }^{\cdot+}\right]\left[\mathrm{M}^{\bullet+}-2 \mathrm{H}\right]: 414.2302$; Found: 414.2310 .

(E)-2-(1-(3,5-Di-tert-butyl-4-hydroxyphenyl)-3-phenylallyl)malononitrile (3bd) and (E)-2-(3-(3,5-Di-tert-butyl-4-hydroxyphenyl)-1-phenylallyl)malononitrile (4bd)

According to GP 1: Starting from $1 \mathbf{b}(15.4 \mathrm{mg}, 0.0480 \mathrm{mmol})$ and $2 \mathrm{~d}-\mathrm{K}(10.0 \mathrm{mg}, 0.0960 \mathrm{mmol})$ two regioisomers were isolated separately after column chromatography (silica gel, pentane/ethyl acetate $=92 / 8$ ) as yellow oils (in total: $11.6 \mathrm{mg}, 63 \%$ ). The 1,6- and 1,8 regioisomers formed in a 42/58 ratio ( ${ }^{1} \mathrm{H}$ NMR spectrum of the crude product mixture). 


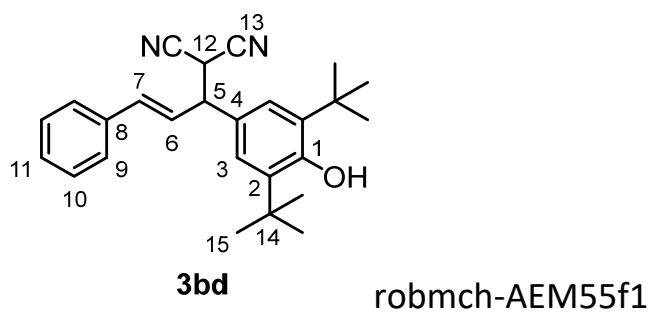

${ }^{1} \mathrm{H}$ NMR (400 MHz, $\left.\mathrm{CDCl}_{3}\right): \delta=7.44-7.41(\mathrm{~m}, 2 \mathrm{H}, 9-\mathrm{H}), 7.37-7.33(\mathrm{~m}, 2 \mathrm{H}, 10-\mathrm{H})$, 7.31-7.27 (m, $1 \mathrm{H}, 11-\mathrm{H}), 7.18(\mathrm{~s}, 2 \mathrm{H}, 3-\mathrm{H}), 6.72(\mathrm{~d}, J=15.7 \mathrm{~Hz}, 1 \mathrm{H}, 7-\mathrm{H}), 6.48$ (dd, J = 15.7 Hz, 7.8 Hz, $1 \mathrm{H}, 6-\mathrm{H}), 5.31$ (s, $1 \mathrm{H}, 1-\mathrm{OH}), 4.06-4.01(\mathrm{~m}, 2 \mathrm{H}, 5-\mathrm{H}, 12-$ $\mathrm{H}), 1.45$ (s, $18 \mathrm{H}, 15-\mathrm{H})$.

${ }^{13} \mathrm{C}$ NMR (101 MHz, CDCl $): \delta=154.3\left(\mathrm{C}_{\mathrm{q}}, \mathrm{C}-1\right), 136.9\left(\mathrm{C}_{\mathrm{q}}, \mathrm{C}-2\right), 135.9\left(\mathrm{C}_{\mathrm{q}}, \mathrm{C}-8\right)$, $135.4(\mathrm{CH}, \mathrm{C}-7), 128.9(\mathrm{CH}, \mathrm{C}-10), 128.6(\mathrm{CH}, \mathrm{C}-11), 127.4\left(\mathrm{C}_{\mathrm{q}}, \mathrm{C}-4\right), 126.9(\mathrm{CH}, \mathrm{C}-$ 9), $124.6(\mathrm{CH}, \mathrm{C}-6), 124.5(\mathrm{CH}, \mathrm{C}-3), 112.1\left(\mathrm{C}_{\mathrm{q}}, \mathrm{C}-13\right), 112.0\left(\mathrm{C}_{\mathrm{q}}, \mathrm{C}-13^{\prime}\right), 50.2(\mathrm{CH}$, $\mathrm{C}-5), 34.7\left(\mathrm{C}_{\mathrm{q}}, \mathrm{C}-14\right), 31.0(\mathrm{CH}, \mathrm{C}-12), 30.3\left(\mathrm{CH}_{3}, \mathrm{C}-15\right)$.

IR (neat): 3630, 2958, 2924, 1723, 1437, 1240, 1157, 966, 750, $693 \mathrm{~cm}^{-1}$.

$\mathrm{HMBC}$ trace for 3-H

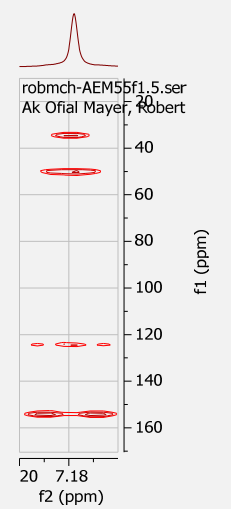<smiles>CC(C)(C)c1cc(/C=C/C(c2ccccc2)C(C#N)C#N)cc(C(C)(C)C)c1O</smiles>

HMBC trace for

${ }^{1} \mathrm{H}$ NMR $\left(599 \mathrm{MHz}, \mathrm{CDCl}_{3}\right): \delta=7.44-7.38(\mathrm{~m}, 5 \mathrm{H}, 9-\mathrm{H}, 10-\mathrm{H}, 11-\mathrm{H}), 7.22(\mathrm{~s}, 2 \mathrm{H}$, 3-H), 6.65 (d, J = $15.7 \mathrm{~Hz}, 1 \mathrm{H}, 5-\mathrm{H}), 6.26$ (dd, J = $15.6 \mathrm{~Hz}, 7.4 \mathrm{~Hz}, 1 \mathrm{H}, 6-\mathrm{H}), 5.32$ (s, $1 \mathrm{H}, 1-\mathrm{OH}), 4.14-4.04$ (m, $2 \mathrm{H}, 7-\mathrm{H}, 12-\mathrm{H}), 1.45$ (s, $18 \mathrm{H}, 15-\mathrm{H})$.

${ }^{13} \mathrm{C} \mathrm{NMR}\left(151 \mathrm{MHz}, \mathrm{CDCl}_{3}\right): \delta=154.7\left(\mathrm{C}_{\mathrm{q}}, \mathrm{C}-1\right), 137.1\left(\mathrm{C}_{\mathrm{q}}, \mathrm{C}-8\right), 136.6(\mathrm{CH}, \mathrm{C}-5)$, $136.4\left(\mathrm{C}_{q}, \mathrm{C}-2\right), 129.5(\mathrm{CH}, \mathrm{C}-10), 129.0(\mathrm{CH}, \mathrm{C}-11), 127.9(\mathrm{CH}, \mathrm{C}-9), 126.9\left(\mathrm{C}_{q}, \mathrm{C}-\right.$ 4), $123.9(\mathrm{CH}, \mathrm{C}-3), 120.8(\mathrm{CH}, \mathrm{C}-6), 111.94\left(\mathrm{C}_{\mathrm{q}}, \mathrm{C}-13\right), 111.88\left(\mathrm{C}_{\mathrm{q}}, \mathrm{C}-13^{\prime}\right), 50.2(\mathrm{CH}$, C-7), $34.5\left(\mathrm{C}_{q}, \mathrm{C}-14\right), 30.4(\mathrm{CH}, \mathrm{C}-12), 30.3\left(\mathrm{CH}_{3}, \mathrm{C}-15\right)$.

HRMS (EI): Calcd $m / z$ for $\left[\mathrm{C}_{26} \mathrm{H}_{30} \mathrm{~N}_{2} \mathrm{O}^{\bullet+}\right]\left[\mathrm{M}^{\bullet+}\right]$ : 386.2353; Found: 386.2363.

IR (neat): 3631, 2957, 2923, 2872, 1723, 1616, 1456, 1438, 1392, 1363, 1239, $1213,1156,1120,1026,965,910,885,758,734,700 \mathrm{~cm}^{-1}$. $3-\mathrm{H}$

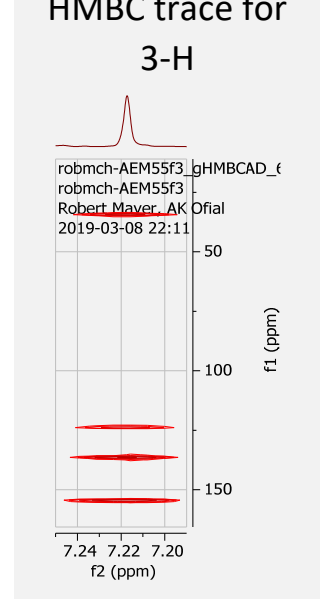

(E)-2-(1-(3,5-Di-tert-butyl-4-hydroxyphenyl)-3-(4-chlorophenyl)allyl)malononitrile (3cd) and (E)-2-(3-(3,5-Di-tert-butyl-4-hydroxyphenyl)-1-(4-chlorophenyl)allyl)malononitrile (4cd)

According to GP 1: Starting from 1c $(17.0 \mathrm{mg}, 0.0479 \mathrm{mmol})$ and $2 \mathrm{~d}-\mathrm{K}(10.0 \mathrm{mg}, 0.0960 \mathrm{mmol})$ a mixture of regioisomeric products $\mathbf{3 c d}$ and $\mathbf{4 c d}$ was isolated after column chromatography (silica, pentane/ethyl acetate $=92 / 8$ to $90 / 10)$ as a yellow oil $(18.2 \mathrm{mg}, 90 \%)$. The 1,6 - and 1,8 regioisomers formed in a $40 / 60$ ratio $\left({ }^{1} \mathrm{H}\right.$ NMR spectrum of the crude product mixture). 

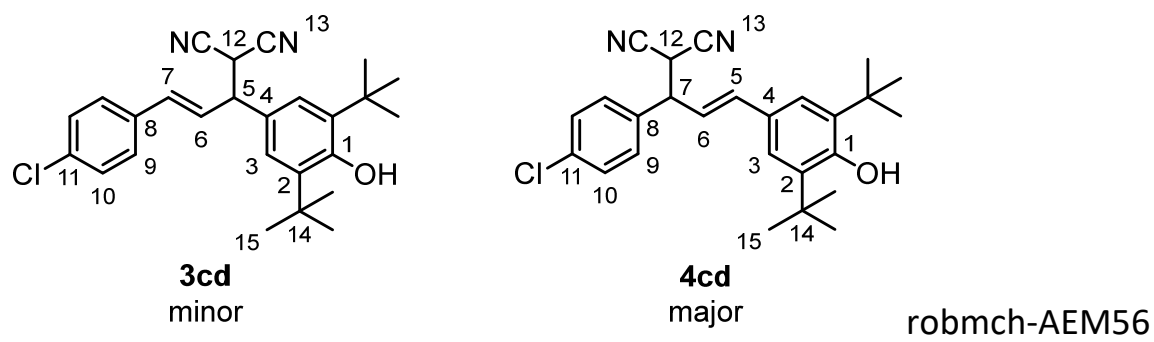

${ }^{1} \mathrm{H}$ NMR $\left(400 \mathrm{MHz}, \mathrm{CDCl}_{3}\right): \delta=7.43-7.40\left(\mathrm{~m}, 2 \mathrm{H}, 10-\mathrm{H}^{1,8}\right), 7.37-7.30(\mathrm{~m}, 6 \mathrm{H}, 10-$ $\left.\mathrm{H}^{1,6}, 9-\mathrm{H}^{1,6}, 9-\mathrm{H}^{1,8}\right), 7.21\left(\mathrm{~s}, 2 \mathrm{H}, 3-\mathrm{H}^{1,8}\right), 7.17\left(\mathrm{~s}, 2 \mathrm{H}, 3-\mathrm{H}^{1,6}\right), 6.68(\mathrm{~d}, \mathrm{~J}=15.7 \mathrm{~Hz}, 1$ $\left.\mathrm{H}, 7-\mathrm{H}^{1,6}\right), 6.63\left(\mathrm{~d}, J=15.7 \mathrm{~Hz}, 1 \mathrm{H}, 5-\mathrm{H}^{1,8}\right), 6.50-6.42\left(\mathrm{~m}, 1 \mathrm{H}, 6-\mathrm{H}^{1,6}\right), 6.21$ (ddd,

HMBC traces for $3-\mathrm{H}^{1,6}$ and $3-\mathrm{H}^{1,8}$

$\left.J=15.7 \mathrm{~Hz}, 7.1 \mathrm{~Hz}, 0.9 \mathrm{~Hz}, 1 \mathrm{H}, 6-\mathrm{H}^{1,8}\right), 5.35\left(\mathrm{~s}, 1 \mathrm{H}, 1-\mathrm{OH}^{1,8}\right), 5.33\left(\mathrm{~s}, 1 \mathrm{H}, 1-\mathrm{OH}^{1,6}\right)$, 4.15-4.01 (m, $\left.4 \mathrm{H}, 5-\mathrm{H}^{1,6}, 12-\mathrm{H}^{1,6}, 7-\mathrm{H}^{1,8}, 12-\mathrm{H}^{1,8}\right), 1.46\left(\mathrm{~s}, 18 \mathrm{H}, 15-\mathrm{H}^{1,6}\right), 1.45$ (s, $\left.18 \mathrm{H}, 15-\mathrm{H}^{1,8}\right)$.

${ }^{13} \mathrm{C}$ NMR (101 MHz, CDCl $): \delta=154.8\left(\mathrm{C}_{\mathrm{q}}, \mathrm{C}-1^{1,8}\right), 154.4\left(\mathrm{C}_{\mathrm{q}}, \mathrm{C}-1^{1,6}\right), 137.0\left(\mathrm{C}_{\mathrm{q}}, \mathrm{C}-\right.$ $\left.2^{1,6}\right), 136.9\left(\mathrm{CH}, \mathrm{C}-5^{1,8}\right), 136.4\left(\mathrm{C}_{\mathrm{q}}, \mathrm{C}-2^{1,8}\right), 135.5\left(\mathrm{C}_{\mathrm{q}}, \mathrm{C}-8^{1,8}\right), 135.0\left(\mathrm{C}, \mathrm{C}-11^{1,8}, \mathrm{C}-\right.$ $\left.11^{1,6}\right), 134.4\left(\mathrm{CH}, \mathrm{C}-7^{1,6}\right), 134.2\left(\mathrm{C}_{q}, \mathrm{C}-8^{1,6}\right), 129.7\left(\mathrm{CH}, \mathrm{C}-10^{1,8}\right), 129.3\left(\mathrm{CH}, \mathrm{C}-9^{1,8}\right)$, $129.0\left(\mathrm{CH}, \mathrm{C}-10^{1,6}\right), 128.1\left(\mathrm{CH}, \mathrm{C}-9^{1,6}\right), 127.2\left(\mathrm{C}_{\mathrm{q}}, \mathrm{C}-4^{1,6}\right), 126.6\left(\mathrm{C}_{\mathrm{q}}, \mathrm{C}-4^{1,8}\right), 125.2$ $\left(\mathrm{CH}, \mathrm{C}-6^{1,6}\right), 124.4\left(\mathrm{CH}, \mathrm{C}-3^{1,6}\right), 123.9\left(\mathrm{CH}, \mathrm{C}-3^{1,8}\right), 120.1\left(\mathrm{CH}, \mathrm{C}-6^{1,8}\right), 112.1\left(\mathrm{C}_{\mathrm{q}}, \mathrm{C}-\right.$ $\left.13^{1,6}\right), 112.0\left(C_{q}, C-13^{1,6^{\prime}}\right), 111.71\left(C_{q}, C-13^{1,8}\right), 111.68\left(C_{q}, C-13^{1,8^{\prime}}\right), 50.2(C H, C-$ $\left.5^{1,6}\right), 49.5\left(\mathrm{CH}, \mathrm{C}-7^{1,8}\right), 34.6\left(\mathrm{C}_{q}, \mathrm{C}-14^{1,6}\right), 34.5\left(\mathrm{C}_{q}, \mathrm{C}-14^{1,8}\right), 30.9$ and $30.3(\mathrm{CH}, \mathrm{C}-$ $12^{1,6}$ and $\left.\mathrm{C}-12^{1,8}\right), 30.32\left(\mathrm{CH}_{3}, \mathrm{C}-15^{1,8}\right), 30.30\left(\mathrm{CH}_{3}, \mathrm{C}-15^{1,6}\right)$.

HRMS (EI $)$ : Calcd $m / z$ for $\left[\mathrm{C}_{26} \mathrm{H}_{29} \mathrm{ClN}_{2} \mathrm{O}^{\bullet+}\right]\left[\mathrm{M}^{\bullet+}\right]$ : 420.1963 ; Found: 420.1957.

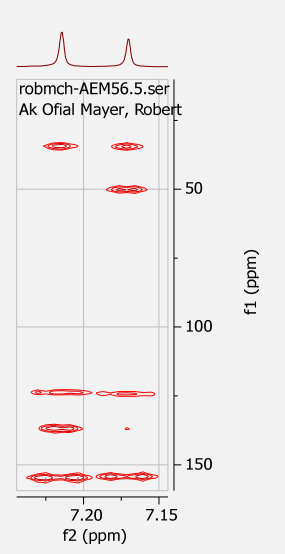

(E)-2-(1-(3,5-Di-tert-butyl-4-hydroxyphenyl)-3-(4-nitrophenyl)allyl)malononitrile (3dd) and (E)-2-(3-(3,5-Di-tert-butyl-4-hydroxyphenyl)-1-(4-nitrophenyl)allyl)malononitrile (4dd)

According to GP 1: Starting from 1d $(17.5 \mathrm{mg}, 0.0479 \mathrm{mmol})$ and $2 \mathrm{~d}-\mathrm{K}(10.0 \mathrm{mg}, 0.0960 \mathrm{mmol})$ a mixture of regioisomeric products $3 \mathbf{d d}$ and $\mathbf{4 d \mathbf { d }}(1,6 / 1,8=70 / 30)$ was isolated after column chromatography (silica, pentane/ethyl acetate $=87 / 13$ ) as a yellow oil $(20.6 \mathrm{mg}, 99 \%)$.<smiles>CC(C#N)[C@H](/C=C/c1ccc([N+](=O)[O-])cc1)c1cc(C(C)(C)C)c(O)c(C(C)(C)C)c1</smiles><smiles>CC(C)(C)c1cc(/C=C/C(c2ccc([N+](=O)[O-])cc2)C(C)(C)C)cc(C(C)(C)C)c1O</smiles> 
${ }^{1} \mathrm{H}$ NMR (400 MHz, $\left.\mathrm{CDCl}_{3}\right): \delta=8.32-8.30\left(\mathrm{~m}, 2 \mathrm{H}, 10-\mathrm{H}^{1,8}\right), 8.23-8.20(\mathrm{~m}, 2 \mathrm{H}$, $\left.10-\mathrm{H}^{1,6}\right)$, 7.63-7.60 (m, $\left.2 \mathrm{H}, 9-\mathrm{H}^{1,8}\right), 7.58-7.55\left(\mathrm{~m}, 2 \mathrm{H}, 9-\mathrm{H}^{1,6}\right), 7.22(\mathrm{~s}, 2 \mathrm{H}, 3-$ $\left.\mathrm{H}^{1,8}\right), 7.17\left(\mathrm{~s}, 2 \mathrm{H}, 3-\mathrm{H}^{1,6}\right), 6.80\left(\mathrm{~d}, J=15.8 \mathrm{~Hz}, 1 \mathrm{H}, 7-\mathrm{H}^{1,6}\right), 6.69-6.62(\mathrm{~m}, 2 \mathrm{H}, 6-$ $\left.\mathrm{H}^{1,6}, 5-\mathrm{H}^{1,8}\right), 6.20\left(\mathrm{dd}, J=15.6 \mathrm{~Hz}, 8.1 \mathrm{~Hz}, 1 \mathrm{H}, 6-\mathrm{H}^{1,8}\right), 5.38\left(\mathrm{~s}, 1 \mathrm{H}, 1-\mathrm{OH}^{1,8}\right), 5.36$ $\left(\mathrm{s}, 1 \mathrm{H}, 1-\mathrm{OH}^{1,6}\right), 4.17-4.06\left(\mathrm{~m}, 4 \mathrm{H}, 5-\mathrm{H}^{1,6}, 12-\mathrm{H}^{1,6}, 7-\mathrm{H}^{1,8}, 12-\mathrm{H}^{1,8}\right), 1.45(\mathrm{~s}, 18 \mathrm{H}$, $\left.15-\mathrm{H}^{1,6}\right), 1.45\left(\mathrm{~s}, 18 \mathrm{H}, 15-\mathrm{H}^{1,8}\right) \cdot{ }^{13} \mathrm{C}$ NMR $\left(101 \mathrm{MHz}, \mathrm{CDCl}_{3}\right): \delta=155.1\left(\mathrm{C}_{\mathrm{q}}, \mathrm{C}-1^{1,8}\right)$, $154.6\left(C_{q}, C-1^{1,6}\right), 148.3\left(C_{q}, C-11^{1,8}\right), 147.7\left(C_{q}, C-11^{1,6}\right), 144.0\left(C_{q}, C-8^{1,8}\right), 142.1$ $\left(\mathrm{C}_{q}, \mathrm{C}-8^{1,6}\right), 137.9\left(\mathrm{CH}, \mathrm{C}-6^{1,6}\right), 137.2\left(\mathrm{C}_{q}, \mathrm{C}-2^{1,6}\right), 136.5\left(\mathrm{C}_{\mathrm{q}}, \mathrm{C}-2^{1,8}\right), 133.3(\mathrm{CH}, \mathrm{C}-$ $\left.7^{1,6}\right), 129.3\left(\mathrm{CH}, \mathrm{C}-5^{1,8}\right), 129.1\left(\mathrm{C}_{\mathrm{q}}, \mathrm{C}-9^{1,8}\right), 127.6\left(\mathrm{CH}, \mathrm{C}-9^{1,6}\right), 126.6\left(\mathrm{C}_{\mathrm{q}}, \mathrm{C}-4^{1,6}\right)$, $126.3\left(\mathrm{C}_{\mathrm{q}}, \mathrm{C}-4^{1,8}\right), 124.7\left(\mathrm{CH}, \mathrm{C}-10^{1,8}\right), 124.4\left(\mathrm{CH}, \mathrm{C}-3^{1,6}\right), 124.3\left(\mathrm{CH}, \mathrm{C}-10^{1,6}\right), 124.0$ $\left(\mathrm{CH}, \mathrm{C}-3^{1,8}\right), 119.0\left(\mathrm{CH}, \mathrm{C}-6^{1,8}\right), 111.9\left(\mathrm{C}_{\mathrm{q}}, \mathrm{C}-13^{1,6}\right), 111.8\left(\mathrm{C}_{\mathrm{q}}, \mathrm{C}-13^{1,6^{\prime}}\right), 111.3\left(\mathrm{C}_{\mathrm{q}}\right.$, $\left.\mathrm{C}-13^{1,8}\right), 50.2\left(\mathrm{CH}, \mathrm{C}-5^{1,6}\right), 49.7\left(\mathrm{CH}, \mathrm{C}-7^{1,8}\right), 34.7\left(\mathrm{C}_{q}, \mathrm{C}-14^{1,6}\right), 34.5\left(\mathrm{C}_{q}, \mathrm{C}-14^{1,8}\right)$, $30.6\left(\mathrm{CH}, \mathrm{C}-12^{1,6}\right), 30.31\left(\mathrm{CH}_{3}, \mathrm{C}-15^{1,8}\right), 30.29\left(\mathrm{CH}_{3}, \mathrm{C}-15^{1,6}\right), 30.0\left(\mathrm{CH}, \mathrm{C}-12^{1,8}\right)$.

HMBC traces for $3-H^{1,6}$ and $3-H^{1,8}$

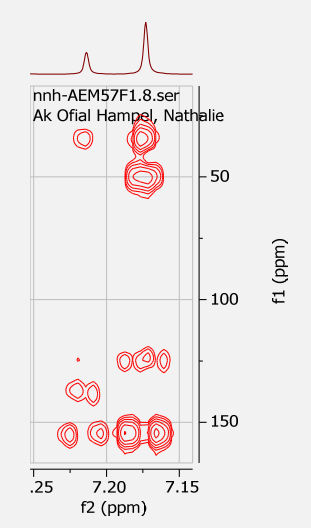

HRMS (El+): Calcd $m / z$ for $\left[\mathrm{C}_{26} \mathrm{H}_{29} \mathrm{~N}_{3} \mathrm{O}_{3}{ }^{*+}\right]\left[\mathrm{M}^{\bullet+}\right]$ : 431.2203; Found: 431.2202.

\section{NMR Study of the Reaction of $1 b$ with $2 d$ (Figure 5)}

The $p$ VQM $1 \mathbf{b}(5.35 \mathrm{mg}, 0.0167 \mathrm{mmol})$ and $2 \mathrm{~d}-\mathrm{K}(1.74 \mathrm{mg}, 0.0167 \mathrm{mmol})$ were mixed in $d_{6}$-DMSO $(0.70$ $\mathrm{mL}$ ) under an atmosphere of dry argon. The resulting reaction mixture was then directly analyzed by NMR spectroscopy without further workup.
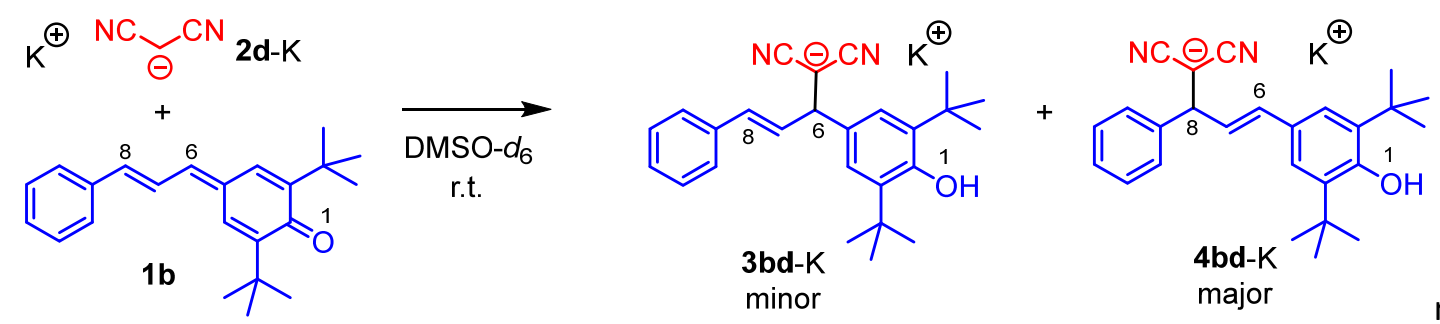

rm1206

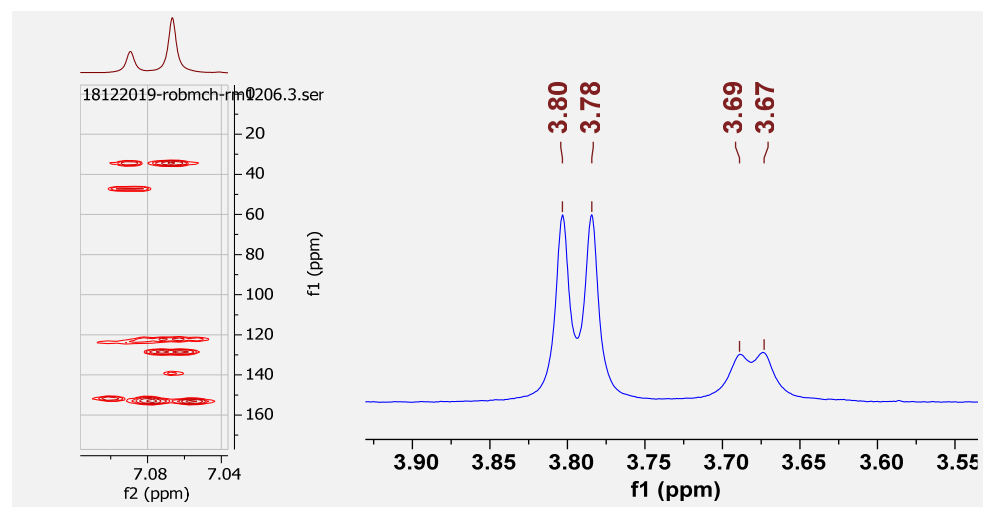

The NMR analytical data show in the HMBC spectrum that a mixture of the 1,6- and 1,8 regioisomers 3bd-K and $\mathbf{4 b d - K}$ formed in an approx. 1:2 ratio. In the ${ }^{1} \mathrm{H}$ NMR spectrum the doublet pattern for both the resonances of $6-\mathrm{H}$ in $3 \mathrm{bd}-\mathrm{K}(\delta=3.68 \mathrm{ppm}, J=6.3 \mathrm{~Hz})$ and $8-\mathrm{H}$ in $4 \mathrm{bd}-\mathrm{K}(\delta=3.79 \mathrm{ppm}, J=7.6 \mathrm{~Hz})$ provide evidence for the presence of deprotonated malononitrile moieties in both products. 


\section{Products of the Reactions of pVQMs 1 with Further Types of Nucleophiles (Table 2)}

\section{2,6-Di-tert-butyl -4-(4-(4-methoxyphenyl)-1,1-bis(phenylsulfonyl)but-3-en-2-yl)phenol (16)}

To a stirred solution of KOtBu (11.3 mg, $0.101 \mathrm{mmol})$ in DMSO $(0.5 \mathrm{~mL})$, a solution of bis(phenylsulfonyl)methane $(29.6 \mathrm{mg}, 0.100 \mathrm{mmol})$ in DMSO $(0.5 \mathrm{~mL})$ was added at room temperature. The $p$ VQM 1a (17.6 mg, $0.05 \mathrm{mmol})$ was dissolved in DMSO $(1 \mathrm{~mL})$ and added to the reaction mixture. The solution was stirred overnight at room temperature and quenched with saturated $\mathrm{NH}_{4} \mathrm{Cl}$ solution $(10 \mathrm{~mL})$. Diethyl ether $(20 \mathrm{~mL})$ was added, the phases were allowed to separate and the aqueous phase was extracted with diethyl ether $(3 \times 20 \mathrm{~mL})$. The combined organic phases were washed with water $(3 \times 20 \mathrm{~mL})$, dried over $\mathrm{Na}_{2} \mathrm{SO}_{4}$, filtered and concentrated in the vacuum to give the crude product. Isolation after column chromatography (silica gel, heptane/ethyl acetate $=10 / 1$ ) gave 16 as a colorless powder (22.0 mg, 68\%); mp 205-207 ${ }^{\circ} \mathrm{C}$.

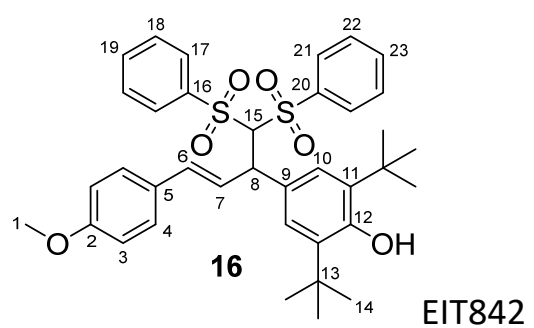

${ }^{1} \mathrm{H}$ NMR $\left(300 \mathrm{MHz}, \mathrm{CDCl}_{3}\right): \delta=7.99-7.96(\mathrm{~m}, 2 \mathrm{H}, 21-\mathrm{H}), 7.63-7.60(\mathrm{~m}, 2 \mathrm{H}, 17-$ H), 7.60-7.56 (m, $1 \mathrm{H}, 23-\mathrm{H}), 7.56-7.51(\mathrm{~m}, 1 \mathrm{H}, 19-\mathrm{H}), 7.47(\mathrm{t}, J=7.7 \mathrm{~Hz}, 2 \mathrm{H}, 22-$ $\mathrm{H}), 7.36(\mathrm{t}, J=7.7 \mathrm{~Hz}, 2 \mathrm{H}, 18-\mathrm{H}), 7.28-7.23(\mathrm{~m}, 2 \mathrm{H}, 4-\mathrm{H}$, superimposed with resonance of residual $\left.\mathrm{CHCl}_{3}\right), 7.07(\mathrm{~s}, 2 \mathrm{H}, 10-\mathrm{H}), 6.88-6.81(\mathrm{~m}, 2 \mathrm{H}, 3-\mathrm{H}), 6.75$ (dd, J = 15.8 Hz, 9.2 Hz, $1 \mathrm{H}, 7-\mathrm{H}), 6.26(\mathrm{~d}, J=15.7 \mathrm{~Hz}, 1 \mathrm{H}, 6-\mathrm{H}), 5.13(\mathrm{~s}, 1 \mathrm{H}, 12-$ $\mathrm{OH}), 5.06(\mathrm{~d}, J=2.9 \mathrm{~Hz}, 1 \mathrm{H}, 15-\mathrm{H}), 4.62(\mathrm{dd}, J=9.3 \mathrm{~Hz}, 3.0 \mathrm{~Hz}, 1 \mathrm{H}, 8-\mathrm{H}), 3.82(\mathrm{~s}$, $3 \mathrm{H}, 1-\mathrm{H}), 1.37(\mathrm{~s}, 18 \mathrm{H}, 14-\mathrm{H})$.

${ }^{13} \mathrm{C}$ NMR (176 MHz, CDCl $\left.)_{3}\right): \delta=159.4\left(C_{q}, C-2\right), 153.0\left(C_{q}, C-12\right), 141.1\left(C_{q}, C-16\right)$, $138.8\left(\mathrm{C}_{\mathrm{q}}, \mathrm{C}-20\right), 136.0\left(\mathrm{C}_{\mathrm{q}}, \mathrm{C}-11\right), 134.4(\mathrm{CH}, \mathrm{C}-23), 134.0(\mathrm{CH}, \mathrm{C}-19), 133.8(\mathrm{CH}$, C-6), 130.7 ( $\left.\mathrm{C}_{q}, \mathrm{C}-9\right), 130.3$ (CH, C-21), 129.8 (C, $\left.\mathrm{C}-5\right), 129.0(\mathrm{CH}, \mathrm{C}-22), 128.81$ ( $\mathrm{CH}, \mathrm{C}-17), 128.77(\mathrm{CH}, \mathrm{C}-18), 128.0(\mathrm{CH}, \mathrm{C}-4) 125.3(\mathrm{CH}, \mathrm{C}-10), 123.6(\mathrm{CH}, \mathrm{C}-7)$, $114.0(\mathrm{CH}, \mathrm{C}-3), 90.1(\mathrm{CH}, \mathrm{C}-15), 55.5\left(\mathrm{CH}_{3}, \mathrm{C}-1\right), 48.3(\mathrm{CH}, \mathrm{C}-8), 34.5\left(\mathrm{C}_{\mathrm{q}}, \mathrm{C}-13\right)$, $30.4\left(\mathrm{CH}_{3}, \mathrm{C}-14\right)$.

HMBC trace for $10-\mathrm{H}$

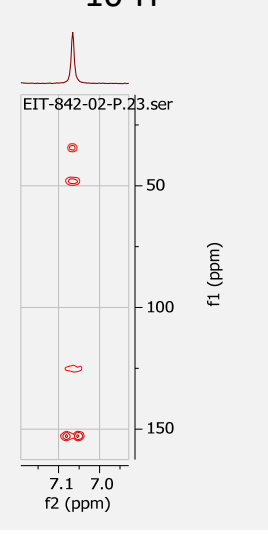

HRMS (ESI): Calcd $m / z$ for $\mathrm{C}_{37} \mathrm{H}_{46} \mathrm{NO}_{6} \mathrm{~S}_{2}\left[\mathrm{M}+\mathrm{NH}_{4}\right]^{+}:$: 664.2761; Found: 664.2765.

IR (neat): 3615, 2955, 2905, 1606, 1510, 1446, 1435, 1333, 1312, 1295, 1266, 1245, 1198, 1176, 1152, $1140,1078,1034,980,936,832,772,752,721,654,627,615 \mathrm{~cm}^{-1}$.

\section{2,6-Di-tert-butyl-4-((12,3E)-4-(4-methoxyphenyl)-1-(phenylsulfonyl)buta-1,3-dien-1-yl)phenol (17)}

To a stirred solution of KOtBu $(11.3 \mathrm{mg}, 0.101 \mathrm{mmol})$ in DMSO $(0.5 \mathrm{~mL})$, a solution of ((chloromethyl)sulfonyl)benzene $(19.1 \mathrm{mg}, 0.100 \mathrm{mmol})$ in DMSO $(0.5 \mathrm{~mL})$ was added. The $p$ VQM 1a (17.6 $\mathrm{mg}, 0.0502 \mathrm{mmol})$ was dissolved in DMSO $(1 \mathrm{~mL})$ and added to the mixture. The reaction mixture 
was stirred overnight at room temperature and quenched with saturated $\mathrm{NH}_{4} \mathrm{Cl}$ solution $(10 \mathrm{~mL})$. Diethyl ether $(20 \mathrm{~mL})$ was added, the phases were separated and the aqueous phase was extracted with diethyl ether $(3 \times 20 \mathrm{~mL})$. The combined organic phases were washed with water $(3 \times 20 \mathrm{~mL})$, dried over $\mathrm{Na}_{2} \mathrm{SO}_{4}$, filtered and concentrated under reduced pressure. The crude product was purified by column chromatography (silica gel, heptane/ethyl acetate $=10 / 1$ ) to furnish $\mathbf{1 7}$ as a yellow powder (16.6 mg, 66\%); mp 70-72 ${ }^{\circ} \mathrm{C}$.

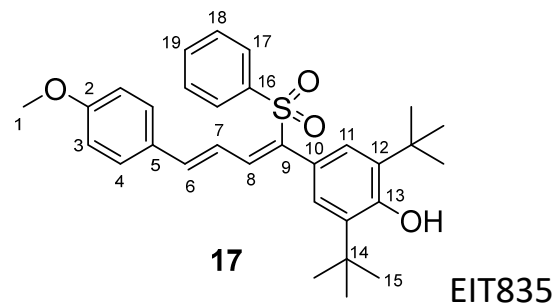

${ }^{1} \mathrm{H}$ NMR $\left(700 \mathrm{MHz}, \mathrm{CDCl}_{3}\right): \delta=7.66(\mathrm{~d}, J=11.2 \mathrm{~Hz}, 1 \mathrm{H}, 8-\mathrm{H}), 7.60(\mathrm{~d}, J=7.0 \mathrm{~Hz}, 2$ $\mathrm{H}, 17-\mathrm{H}$ ), 7.50 (app t, J = 7.5 Hz, $1 \mathrm{H}, 19-\mathrm{H}), 7.39-7.38(\mathrm{~m}, 2 \mathrm{H}, 18-\mathrm{H}), 7.29$ (d, J = $8.9 \mathrm{~Hz}, 2 \mathrm{H}, 4-\mathrm{H}), 6.99(\mathrm{~d}, J=15.4 \mathrm{~Hz}, 1 \mathrm{H}, 6-\mathrm{H}), 6.83(\mathrm{~d}, J=8.8 \mathrm{~Hz}, 2 \mathrm{H}, 3-\mathrm{H}), 6.80$ (s, $2 \mathrm{H}, 11-\mathrm{H}), 6.49$ (dd, J = 15.5, $11.2 \mathrm{~Hz}, 1 \mathrm{H}, 7-\mathrm{H}), 5.31(\mathrm{~s}, 1 \mathrm{H}, 13-\mathrm{OH}), 3.80$ (s, $3 \mathrm{H}, 1-\mathrm{H}), 1.33(\mathrm{~s}, 18 \mathrm{H}, 15-\mathrm{H})$.

${ }^{13} \mathrm{C}$ NMR (176 MHz, CDCl $): \delta=160.6\left(\mathrm{C}_{\mathrm{q}}, \mathrm{C}-2\right), 154.5\left(\mathrm{C}_{\mathrm{q}}, \mathrm{C}-13\right), 141.7(\mathrm{CH}, \mathrm{C}-6)$, $141.0\left(\mathrm{C}_{\mathrm{q}}, \mathrm{C}-9\right), 140.1$ ( $\left.\mathrm{C}_{\mathrm{q}}, \mathrm{C}-16\right), 138.2(\mathrm{CH}, \mathrm{C}-8), 135.8\left(\mathrm{C}_{\mathrm{q}}, \mathrm{C}-12\right), 132.8(\mathrm{CH}, \mathrm{C}-$ 19), $129.1\left(\mathrm{C}_{q}, \mathrm{C}-5\right), 128.9(\mathrm{CH}, \mathrm{C}-4), 128.7(\mathrm{CH}, \mathrm{C}-18), 128.5(\mathrm{CH}, \mathrm{C}-17), 128.1(\mathrm{CH}$, $\mathrm{C}-11), 121.8\left(\mathrm{C}_{\mathrm{q}}, \mathrm{C}-10\right), 121.3(\mathrm{CH}, \mathrm{C}-7), 114.4(\mathrm{CH}, \mathrm{C}-3), 55.5\left(\mathrm{CH}_{3}, \mathrm{C}-1\right), 34.4\left(\mathrm{C}_{\mathrm{q}}\right.$, $\mathrm{C}-14), 30.4\left(\mathrm{CH}_{3}, \mathrm{C}-15\right)$.

HRMS (ESI): Calcd $m / z$ for $\mathrm{C}_{31} \mathrm{H}_{40} \mathrm{NO}_{4} \mathrm{~S}\left[\mathrm{M}+\mathrm{NH}_{4}\right]^{+}:$522.2673; Found: 522.2671.

IR (neat): 3619, 2959, 2894, 2366, 2320, 1598, 1568, 1509, 1473, 1438, 1367,

HMBC trace for $11-\mathrm{H}$

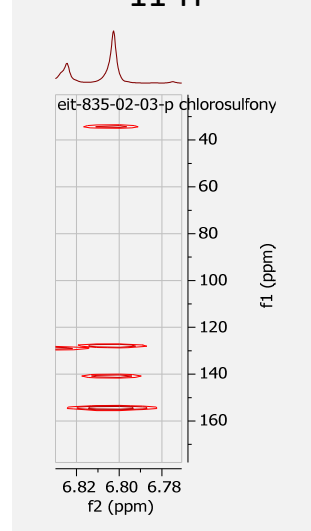
$1302,1259,1239,1183,1171,1139,1120,1081,1028,975,898,870,848,809,757,735,716,691$, $659,601 \mathrm{~cm}^{-1}$.

\section{1-(3-(3,5-Di-tert-butyl-4-hydroxyphenyl)-1-ethoxy-1-oxo-5-phenylpent-4-en-2-yl)pyridin-1-ium bromide (18)}

The $p$ VQM $1 \mathbf{b}$ ( $32.0 \mathrm{mg}, 0.100 \mathrm{mmol}$ ) and 1-(2-ethoxy-2-oxoethyl)pyridinium bromide ( $24.6 \mathrm{mg}, 0.100$ $\mathrm{mmol})$ were mixed in acetonitrile $(3.3 \mathrm{~mL})$ and stirred for $5 \mathrm{~min}$. Triethylamine $(31 \mu \mathrm{L}, 0.22 \mathrm{mmol})$ was added, and the reaction mixture was stirred overnight at room temperature. The crude product was obtained after filtration through $\mathrm{Na}_{2} \mathrm{SO}_{4}$ (washed with $20 \mathrm{~mL}$ of dichloromethane) and evaporation of the solvent. Preparative TLC gave a 64/36-mixture of the diastereomers $\mathbf{1 8}$ and $\mathbf{1 8}$ ' as a viscous oil ( $33.3 \mathrm{mg}, 60 \%$ ). The diastereomers were separated by preparative HPLC and characterized individually.

Diastereomer 18 was isolated after semi-preparative HPLC (silica gel, dichloromethane/isopropanol = $9 / 1) ; R_{\mathrm{f}}$ (dichloromethane/isopropanol $=5 / 1$ ) $=0.40$. 


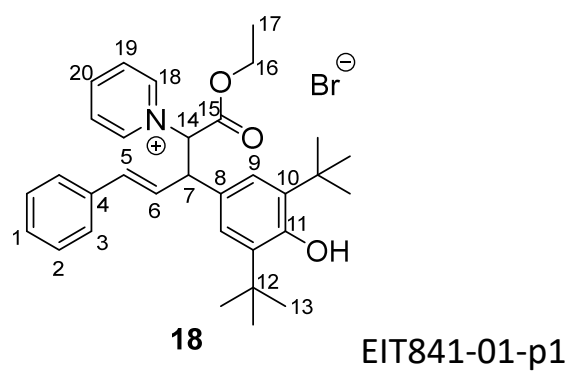

${ }^{1} \mathrm{H}$ NMR $\left(700 \mathrm{MHz}, \mathrm{CDCl}_{3}\right): \delta=10.15-10.14(\mathrm{~m}, 2 \mathrm{H}, 18-\mathrm{H}), 8.37(\mathrm{t}, J=7.7 \mathrm{~Hz}, 1 \mathrm{H}$, 20-H), 7.99 (t, J = 7.0 Hz, $2 \mathrm{H}, 19-\mathrm{H}), 7.37(\mathrm{~s}, 2 \mathrm{H}, 9-\mathrm{H}), 7.29$ (d, J= 7.4 Hz, $2 \mathrm{H}, 3-$ H), 7.22-7.19 (m, $3 \mathrm{H}, 2-\mathrm{H}, 14-\mathrm{H}), 7.17-7.14(\mathrm{~m}, 1 \mathrm{H}, 1-\mathrm{H}), 7.00$ (dd, J = $15.7 \mathrm{~Hz}$, $9.6 \mathrm{~Hz}, 1 \mathrm{H}, 6-\mathrm{H}), 6.18(\mathrm{~d}, J=15.7 \mathrm{~Hz}, 1 \mathrm{H}, 5-\mathrm{H}), 5.31(\mathrm{~s}, 1 \mathrm{H}, 11-\mathrm{OH}), 4.01(\mathrm{t}, J=$ $10.0 \mathrm{~Hz}, 1 \mathrm{H}, 7-\mathrm{H}), 4.01-3.96(\mathrm{~m}, 1 \mathrm{H}, 16-\mathrm{H}), 3.91-3.87(\mathrm{~m}, 1 \mathrm{H}, 16-\mathrm{H}), 1.47(\mathrm{~s}, 18$ $\mathrm{H}, 13-\mathrm{H}), 0.85(\mathrm{t}, J=7.1 \mathrm{~Hz}, 3 \mathrm{H}, 17-\mathrm{H})$.

${ }^{13} \mathrm{C}$ NMR (176 MHz, CDCl $): \delta=168.1\left(\mathrm{C}_{\mathrm{q}}, \mathrm{C}-15\right), 154.1\left(\mathrm{C}_{\mathrm{q}}, \mathrm{C}-11\right), 145.8(\mathrm{CH}, \mathrm{C}-$ 20), $145.6(\mathrm{CH}, \mathrm{C}-18), 137.0\left(\mathrm{C}_{\mathrm{q}}, \mathrm{C}-10\right), 135.8\left(\mathrm{C}_{\mathrm{q}}, \mathrm{C}-4\right), 133.7(\mathrm{CH}, \mathrm{C}-5), 128.7(\mathrm{CH}$, $\mathrm{C}-2), 128.2(\mathrm{CH}, \mathrm{C}-1), 127.6(\mathrm{CH}, \mathrm{C}-19), 126.9(\mathrm{CH}, \mathrm{C}-3), 126.7\left(\mathrm{C}_{q}, \mathrm{C}-8\right), 126.4(\mathrm{CH}$, $\mathrm{C}-6), 125.5(\mathrm{CH}, \mathrm{C}-9), 73.4(\mathrm{CH}, \mathrm{C}-14), 63.1\left(\mathrm{CH}_{2}, \mathrm{C}-16\right), 57.6(\mathrm{CH}, \mathrm{C}-7), 34.8(\mathrm{C}, \mathrm{C}-$ 12), $30.6\left(\mathrm{CH}_{3}, \mathrm{C}-13\right), 13.5\left(\mathrm{CH}_{3}, \mathrm{C}-17\right)$.

HRMS (ESI): Calcd $m / z$ for $\mathrm{C}_{32} \mathrm{H}_{40} \mathrm{NO}_{3}{ }^{+}[\mathrm{M}]^{+}$: 486.3003; Found: 486.3002 .

$H M B C$ trace for $9-\mathrm{H}$

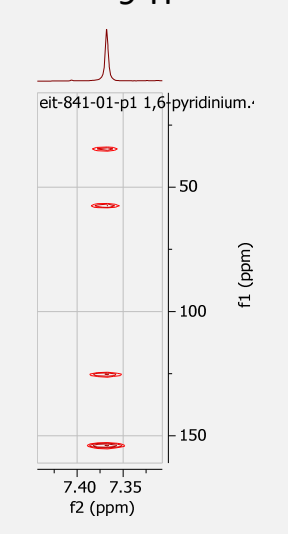

IR (film): 3632, 2955, 2924, 2856, 2361, 2341, 1736, 1630, 1484, 1435, 1395, 1369, 1326, 1267, 1207, $1187,1160,1122,1097,1014,986,853,732,697,681,645,617 \mathrm{~cm}^{-1}$.

Diastereomer 18' was isolated after semi-preparative HPLC (silica gel, dichloromethane/isopropanol = 9/1); $R_{\mathrm{f}}$ (dichloromethane/isopropanol $=5 / 1$ ) $=0.40$.

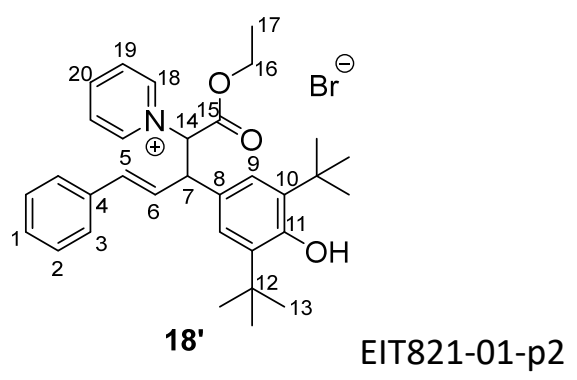

${ }^{1} \mathrm{H} \mathrm{NMR}\left(700 \mathrm{MHz}, \mathrm{CDCl}_{3}\right): \delta=9.77(\mathrm{br} \mathrm{d}, J=6.0 \mathrm{~Hz}, 2 \mathrm{H}, 18-\mathrm{H}), 8.29(\mathrm{t}, J=7.6 \mathrm{~Hz}$, $1 \mathrm{H}, 20-\mathrm{H}), 7.84(\mathrm{t}, J=6.9 \mathrm{~Hz}, 2 \mathrm{H}, 19-\mathrm{H}), 7.42(\mathrm{~d}, J=7.3 \mathrm{~Hz}, 2 \mathrm{H}, 3-\mathrm{H}), 7.33(\mathrm{t}, J=$ $7.6 \mathrm{~Hz}, 2 \mathrm{H}, 2-\mathrm{H}), 7.28-7.25(\mathrm{~m}, 1 \mathrm{H}, 1-\mathrm{H}$, superimposed by resonance for residual $\left.\mathrm{CHCl}_{3}\right), 7.15(\mathrm{~d}, J=9.9 \mathrm{~Hz}, 1 \mathrm{H}, 14-\mathrm{H}), 6.95(\mathrm{~s}, 2 \mathrm{H}, 9-\mathrm{H}), 6.71$ (dd, $J=15.7 \mathrm{~Hz}, 8.6$ $\mathrm{Hz}, 1 \mathrm{H}, 6-\mathrm{H}), 6.63(\mathrm{~d}, J=15.8 \mathrm{~Hz}, 1 \mathrm{H}, 5-\mathrm{H}), 5.23(\mathrm{~s}, 1 \mathrm{H}, 11-\mathrm{OH}), 4.31-4.27(\mathrm{~m}, 1$ $\mathrm{H}, 16-\mathrm{H}), 4.24-4.19(\mathrm{~m}, 1 \mathrm{H}, 16-\mathrm{H}), 4.19(\mathrm{t}, J=9.3 \mathrm{~Hz}, 7-\mathrm{H}), 1.36(\mathrm{~s}, 18 \mathrm{H}, 13-\mathrm{H})$, $1.21(\mathrm{t}, J=7.1 \mathrm{~Hz}, 3 \mathrm{H}, 17-\mathrm{H})$.

${ }^{13} \mathrm{C}$ NMR (176 MHz, CDCl $): \delta=168.0\left(\mathrm{C}_{\mathrm{q}}, \mathrm{C}-15\right), 153.9\left(\mathrm{C}_{\mathrm{q}}, \mathrm{C}-11\right), 146.0(\mathrm{CH}, \mathrm{C}-$ 18), $145.3(\mathrm{CH}, \mathrm{C}-20), 137.1\left(\mathrm{C}_{\mathrm{q}}, \mathrm{C}-10\right), 136.1(\mathrm{C}, \mathrm{C}-4), 134.5(\mathrm{CH}, \mathrm{C}-5), 128.8(\mathrm{CH}$, C-2), $128.4(\mathrm{CH}, \mathrm{C}-1), 127.0$ (CH, C-19), 126.9 (CH, C-3), 126.7 (C, C-10), 125.3 $(\mathrm{CH}, \mathrm{C}-8), 124.7(\mathrm{CH}, \mathrm{C}-9), 73.9(\mathrm{CH}, \mathrm{C}-14), 63.7\left(\mathrm{CH}_{2}, \mathrm{C}-16\right), 55.9(\mathrm{CH}, \mathrm{C}-7), 34.6$ $(\mathrm{C}, \mathrm{C}-12), 30.4\left(\mathrm{CH}_{3}, \mathrm{C}-13\right), 14.1\left(\mathrm{CH}_{3}, \mathrm{C}-17\right)$.

HMBC trace for 9-H

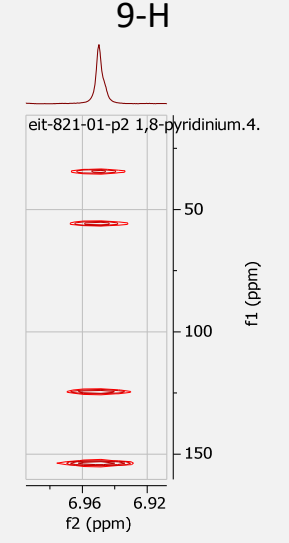
HRMS (ESI): Calcd $m / z$ for $\mathrm{C}_{32} \mathrm{H}_{40} \mathrm{NO}_{3}{ }^{+}[\mathrm{M}]^{+}:$486.3003; Found: 486.3002. 
IR (film): 3629, 2955, 2871, 2360, 2341, 1737, 1630, 1578, 1485, 1434, 1392, 1367, 1266, 1188, 1159, $1121,1027,968,887,845,773,733,694,620 \mathrm{~cm}^{-1}$.

\section{2,6-Di-tert-butyl-4-cinnamylphenol (19)}

The $p$ VQM $1 \mathbf{b}(32.1 \mathrm{mg}, 0.100 \mathrm{mmol})$ was dissolved in methanol $(2 \mathrm{~mL})$. Then $\mathrm{NaBH}_{4}(8.4 \mathrm{mg}, 0.22$ $\mathrm{mmol}$ ) was added portionwise over $30 \mathrm{~min}$ at room temperature. The reaction mixture was allowed to stir for $1 \mathrm{~h}$ at room temperature, cooled to $0{ }^{\circ} \mathrm{C}$, and quenched with saturated $\mathrm{NH}_{4} \mathrm{Cl}$ solution $(10 \mathrm{~mL})$. The mixture was extracted with dichloromethane $(3 \times 20 \mathrm{~mL})$, the combined organic layers were dried over $\mathrm{Na}_{2} \mathrm{SO}_{4}$, filtered, and the solvent was evaporated under reduced pressure. The crude product was purified by column chromatography (silica gel, heptane/ethyl acetate $=10 / 1$ ) to yield 19 as a colorless powder (29.4 $\mathrm{mg}, 91 \%)$; $\mathrm{mp} 92-94^{\circ} \mathrm{C}$.

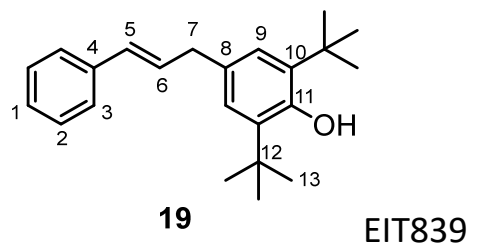

${ }^{1} \mathrm{H} \mathrm{NMR}\left(700 \mathrm{MHz}, \mathrm{CDCl}_{3}\right): \delta=7.38(\mathrm{~d}, J=7.4 \mathrm{~Hz}, 2 \mathrm{H}, 3-\mathrm{H}), 7.30$ (app t, $J=7.7 \mathrm{~Hz}$, $2 \mathrm{H}, 2-\mathrm{H}$ ), 7.21 (app t, J = 7.5 Hz, $1 \mathrm{H}, 1-\mathrm{H}), 7.05(\mathrm{~s}, 2 \mathrm{H}, 9-\mathrm{H}), 6.47(\mathrm{~d}, J=15.7 \mathrm{~Hz}$, $1 \mathrm{H}, 5-\mathrm{H}), 6.37(\mathrm{dt}, J=15.8 \mathrm{~Hz}, 7.0 \mathrm{~Hz}, 1 \mathrm{H}, 6-\mathrm{H}), 5.09(\mathrm{~s}, 1 \mathrm{H}, 11-\mathrm{OH}), 3.48(\mathrm{~d}, J=$ $7.0 \mathrm{~Hz}, 2 \mathrm{H}, 7-\mathrm{H}), 1.45$ (s, $18 \mathrm{H}, 13-\mathrm{H})$.

${ }^{13} \mathrm{C}$ NMR (176 MHz, CDCl 3$): \delta=152.3\left(\mathrm{C}_{\mathrm{q}}, \mathrm{C}-11\right), 137.9\left(\mathrm{C}_{\mathrm{q}}, \mathrm{C}-4\right), 136.1\left(\mathrm{C}_{\mathrm{q}}, \mathrm{C}-10\right)$, $130.9\left(\mathrm{C}_{\mathrm{q}}, \mathrm{C}-8\right), 130.5$ ( $\left.\mathrm{CH}, \mathrm{C}-6\right), 130.3(\mathrm{CH}, \mathrm{C}-5), 128.6(\mathrm{CH}, \mathrm{C}-2), 127.1(\mathrm{CH}, \mathrm{C}-1)$, $126.3(\mathrm{CH}, \mathrm{C}-3), 125.3(\mathrm{CH}, \mathrm{C}-9), 39.6\left(\mathrm{CH}_{2}, \mathrm{C}-7\right), 34.5(\mathrm{C}, \mathrm{C}-12), 30.5\left(\mathrm{CH}_{3}, \mathrm{C}-13\right)$. HRMS (ESI): Calcd $m / z$ for $\mathrm{C}_{23} \mathrm{H}_{31} \mathrm{O}[\mathrm{M}+\mathrm{H}]^{+}:$323.2369; Found: 323.2381.

IR (neat): 3624, 2950, 2909, 1641, 1632, 1589, 1432, 1360, 1310, 1235, 1198, $1183,1146,1120,968,935,885,875,770,742,692,643 \mathrm{~cm}^{-1}$.

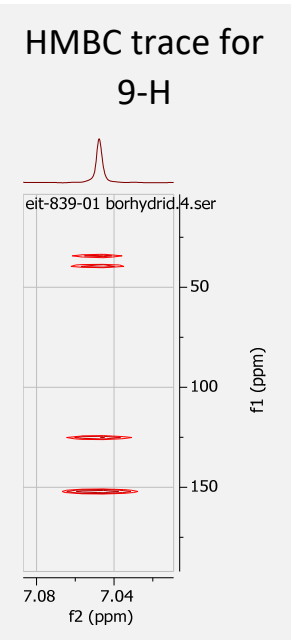

\section{(E)-2,6-Di-tert-butyl-4-(3-(4-chlorophenyl)-3-(pyrrolidin-1-yl)prop-1-en-1-yl)phenol (20)}

The $p$ VQM 1c ( $35.5 \mathrm{mg}, 0.100 \mathrm{mmol})$ was dissolved in DMSO (1 mL). Pyrrolidine $(8.5 \mu \mathrm{L}, 0.20 \mathrm{mmol})$ was added and the solution was stirred overnight at room temperature. The reaction mixture was extracted with diethyl ether $(5 \times 15 \mathrm{~mL})$ and the combined ether phases were washed with water $(3 \times$ $20 \mathrm{~mL}$ ). The organic phase was dried over $\mathrm{Na}_{2} \mathrm{SO}_{4}$, filtered and concentrated in the vacuum. The crude product was purified by column chromatography (silica gel, heptane/ethyl acetate $=5 / 1, R_{\mathrm{f}}=0.55$ ) to give 20 as a light red oil (11.4 mg, 54\%).<smiles>CCC(C)(C)c1cc(/C=C/N(c2ccc(Cl)cc2)C2CCCC2)cc(C(C)(C)C)c1O</smiles> 
${ }^{1} \mathrm{H} \mathrm{NMR}\left(700 \mathrm{MHz}, \mathrm{CDCl}_{3}\right): \delta=7.35(\mathrm{~d}, J=8.2 \mathrm{~Hz}, 2 \mathrm{H}, 3-\mathrm{H}), 7.28(\mathrm{~d}, J=8.2 \mathrm{~Hz}, 2$ $\mathrm{H}, 2-\mathrm{H}), 7.17(\mathrm{~s}, 2 \mathrm{H}, 9-\mathrm{H}), 6.49$ (d, J = $15.6 \mathrm{~Hz}, 1 \mathrm{H}, 7-\mathrm{H}), 6.18$ (dd, $J=15.7 \mathrm{~Hz}, 8.7$ $\mathrm{Hz}, 1 \mathrm{H}, 6-\mathrm{H}), 5.20(\mathrm{~s}, 1 \mathrm{H}, 11-\mathrm{OH}), 3.71(\mathrm{~d}, J=8.7 \mathrm{~Hz}, 1 \mathrm{H}, 5-\mathrm{H}), 2.56-2.42(\mathrm{~m}, 4$ $\mathrm{H}, 14-\mathrm{H}), 1.80-1.77(\mathrm{~m}, 4 \mathrm{H}, 15-\mathrm{H}), 1.42(\mathrm{~s}, 18 \mathrm{H}, 13-\mathrm{H})$.

${ }^{13} \mathrm{CNMR}\left(176 \mathrm{MHz}, \mathrm{CDCl}_{3}\right): \delta=153.8\left(\mathrm{C}_{\mathrm{q}}, \mathrm{C}-11\right), 142.2\left(\mathrm{C}_{\mathrm{q}}, \mathrm{C}-4\right), 136.1\left(\mathrm{C}_{\mathrm{q}}, \mathrm{C}-10\right)$, $132.6\left(\mathrm{C}_{\mathrm{q}}, \mathrm{C}-1\right), 131.0(\mathrm{CH}, \mathrm{C}-7), 129.6(\mathrm{CH}, \mathrm{C}-6), 129.0(\mathrm{CH}, \mathrm{C}-3), 128.8(\mathrm{CH}, \mathrm{C}-2)$, $128.0\left(\mathrm{C}_{\mathrm{q}}, \mathrm{C}-8\right), 123.4(\mathrm{CH}, \mathrm{C}-9), 74.0(\mathrm{CH}, \mathrm{C}-5), 53.3\left(\mathrm{CH}_{2}, \mathrm{C}-14\right), 34.5\left(\mathrm{C}_{q}, \mathrm{C}-12\right)$, $30.4\left(\mathrm{CH}_{3}, \mathrm{C}-13\right), 23.5\left(\mathrm{CH}_{2}, \mathrm{C}-15\right)$; slow decomposition of 20 in $\mathrm{CDCl}_{3}$ gave rise to several additional resonances in the ${ }^{13} \mathrm{C}$ NMR spectrum.

HRMS (ESI): Calcd $m / z$ for $\mathrm{C}_{27} \mathrm{H}_{37} \mathrm{CINO}[\mathrm{M}+\mathrm{H}]^{+}$: 426.2558; Found: 426.2568.

IR (film): 3633, 2955, 2872, 2788, 2360, 2341, 1610, 1590, 1561, 1539, 1487, $1435,1389,1360,1264,1252,1234,1153,1119,1090,1013,964,950,884,865$,

HMBC trace for $9-\mathrm{H}$

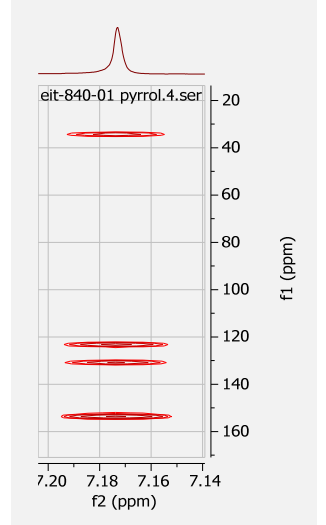
$810,769,702 \mathrm{~cm}^{-1}$.

\section{(E)-2,6-Di-tert-butyl-4-(3-(4-chlorophenyl)-3-(methylthio)prop-1-en-1-yl)phenol (21)}

Sodium methanethiolate $(7.8 \mathrm{mg}, 0.11 \mathrm{mmol})$ was suspended in DMSO $(1 \mathrm{~mL})$, a solution of the $p$ VQM 1c $(17.7 \mathrm{mg}, 0.050 \mathrm{mmol})$ in DMSO $(1 \mathrm{~mL})$ was added and the mixture was stirred overnight at room temperature. The reaction mixture was extracted with diethyl ether $(5 \times 15 \mathrm{~mL})$ and the combined ether phases were washed with water $(3 \times 20 \mathrm{~mL})$. The organic phase was dried over $\mathrm{Na}_{2} \mathrm{SO}_{4}$, filtered and concentrated under reduced pressure. The crude product was purified by column chromatography (silica gel, heptane/ethyl acetate $=10 / 1$ ) to give 21 as a yellowish oil $(15.9 \mathrm{mg}, 79 \%) ; R_{\mathrm{f}}$ (heptane/ethyl acetate $=5 / 1)=0.65$.

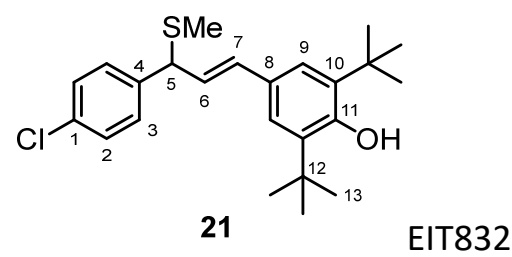

${ }^{1} \mathrm{H} \mathrm{NMR}\left(700 \mathrm{MHz}, \mathrm{CDCl}_{3}\right): \delta=7.36(\mathrm{~d}, J=8.5 \mathrm{~Hz}, 2 \mathrm{H}, 3-\mathrm{H}), 7.31(\mathrm{~d}, J=8.5 \mathrm{~Hz}, 2 \mathrm{H}$, 2-H), $7.21(\mathrm{~s}, 2 \mathrm{H}, 9-\mathrm{H}), 6.43(\mathrm{~d}, J=15.5 \mathrm{~Hz}, 1 \mathrm{H}, 7-\mathrm{H}), 6.13$ (dd, J=15.6 Hz, 8.6 Hz, $1 \mathrm{H}, 6-\mathrm{H}), 5.25(\mathrm{~s}, 1 \mathrm{H}, 11-\mathrm{OH}), 4.45(\mathrm{~d}, J=8.6 \mathrm{~Hz}, 1 \mathrm{H}, 5-\mathrm{H}), 2.06(\mathrm{~s}, 3 \mathrm{H}, \mathrm{SMe}), 1.44$ (s, $18 \mathrm{H}, 13-\mathrm{H})$.

${ }^{13} \mathrm{C}$ NMR (176 MHz, CDCl 3$): \delta=154.1\left(\mathrm{C}_{q}, \mathrm{C}-11\right), 139.6\left(\mathrm{C}_{\mathrm{q}}, \mathrm{C}-4\right), 136.2\left(\mathrm{C}_{\mathrm{q}}, \mathrm{C}-10\right)$, $133.1\left(\mathrm{C}_{\mathrm{q}}, \mathrm{C}-1\right), 132.5(\mathrm{CH}, \mathrm{C}-7), 129.4(\mathrm{CH}, \mathrm{C}-3), 128.9(\mathrm{CH}, \mathrm{C}-2), 127.7\left(\mathrm{C}_{\mathrm{q}}, \mathrm{C}-8\right)$, $125.7(\mathrm{CH}, \mathrm{C}-6), 123.5(\mathrm{CH}, \mathrm{C}-9), 53.8(\mathrm{CH}, \mathrm{C}-5), 34.5(\mathrm{C}, \mathrm{C}-12), 30.4\left(\mathrm{CH}_{3}, \mathrm{C}-13\right)$, $15.4\left(\mathrm{CH}_{3}, \mathrm{SMe}\right)$.

HRMS (ESI): Calcd $m / z$ for $\mathrm{C}_{24} \mathrm{H}_{32} \mathrm{ClOS}[\mathrm{M}+\mathrm{H}]^{+}$: 403.1857; Found: 403.1865.

IR (Iq. film): 3630, 2955, 2915, 2869, 2360, 2341, 1610, 1589, 1561, 1539, 1488, $1455,1434,1389,1360,1314,1253,1236,1135,1119,1091,1012,964,950,884$, $810,737,703,689 \mathrm{~cm}^{-1}$.

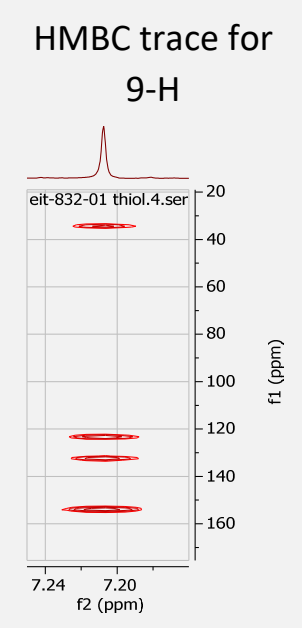




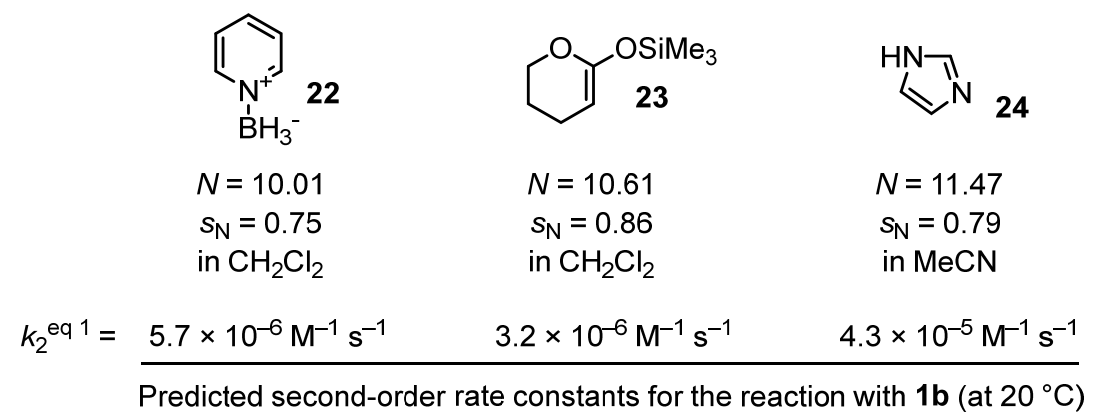

(1) The $p$ VQM 1b $(5.3 \mathrm{mg}, 0.016 \mathrm{mmol}$ ) and the pyridine-borane complex $22(3.1 \mathrm{mg}, 0.033 \mathrm{mmol})$ were dissolved in $\mathrm{CD}_{2} \mathrm{Cl}_{2}(0.75 \mathrm{~mL})$. The mixture was left at room temperature over a period of 4 days. ${ }^{1} \mathrm{H}$ NMR spectra acquired during this time period remained unchanged and showed that $\mathbf{1 b}$ did not react with 22 (RNH 430).

(2) The pVQM 1b $(5.4 \mathrm{mg}, 0.017 \mathrm{mmol})$ and the silyl ketene acetal $23(5.9 \mathrm{mg}, 0.034 \mathrm{mmol})$ were dissolved in $\mathrm{CD}_{2} \mathrm{Cl}_{2}(0.75 \mathrm{~mL})$. The mixture was left at room temperature over a period of 4 days. ${ }^{1} \mathrm{H}$ NMR spectra acquired during this reaction time showed that $\mathbf{1 b}$ did not react with $\mathbf{2 3}$, and only slow degradation of $\mathbf{2 3}$ by desilylation was observed (RNH 428).

(3) The $p$ VQM $1 \mathbf{b}$ (5.3 $\mathrm{mg}, 0.016 \mathrm{mmol}$ ) and imidazole $24(2.3 \mathrm{mg}, 0.034 \mathrm{mmol})$ were dissolved in $\mathrm{CD}_{3} \mathrm{CN}$ $(0.75 \mathrm{~mL})$. The mixture was left at room temperature over a period of 4 days. ${ }^{1} \mathrm{H}$ NMR spectra acquired during this reaction time showed that only a small part of $\mathbf{1 b}(<20 \%)$ was consumed in a reaction with the nucleophile (RNH431). 


\section{Kinetics}

\section{Reactions of $p$ VQM 1a $(\mathrm{R}=\mathrm{OMe})$ with Reference Nucleophiles}

$1 a+2 a$ in DMSO (Stopped-flow, detection at $432 \mathrm{~nm}$ ) AEM-45

\begin{tabular}{llllll}
\hline$[\mathbf{1 a}]_{0}$, & {$[\mathbf{2 a}-\mathrm{H}]_{0}$,} & {$[\mathrm{KOtBu}]_{0}$,} & {$[18-\mathrm{c}-6]_{0}$,} & {$[\mathbf{2 a}]_{0}$,} & $k_{\text {obs, }}$ \\
$\mathrm{M}$ & $\mathrm{M}$ & $\mathrm{M}$ & $\mathrm{M}$ & $\mathrm{M}$ & $\mathrm{s}^{-1}$ \\
\hline $1.20 \times 10^{-5}$ & $4.00 \times 10^{-4}$ & $2.00 \times 10^{-4}$ & & $2.00 \times 10^{-4}$ & $8.48 \times 10^{-2}$ \\
$1.20 \times 10^{-5}$ & $6.00 \times 10^{-4}$ & $3.00 \times 10^{-4}$ & $3.00 \times 10^{-4}$ & $3.00 \times 10^{-4}$ & $1.59 \times 10^{-1}$ \\
$1.20 \times 10^{-5}$ & $8.00 \times 10^{-4}$ & $4.00 \times 10^{-4}$ & & $4.00 \times 10^{-4}$ & $2.18 \times 10^{-1}$ \\
$1.20 \times 10^{-5}$ & $1.00 \times 10^{-3}$ & $5.00 \times 10^{-4}$ & $5.00 \times 10^{-4}$ & $5.00 \times 10^{-4}$ & $2.55 \times 10^{-1}$ \\
$1.20 \times 10^{-5}$ & $1.20 \times 10^{-3}$ & $6.00 \times 10^{-4}$ & & $6.00 \times 10^{-4}$ & $3.23 \times 10^{-1}$ \\
\hline
\end{tabular}

$$
k_{2}=5.72 \times 10^{2} \mathrm{M}^{-1} \mathrm{~s}^{-1}
$$

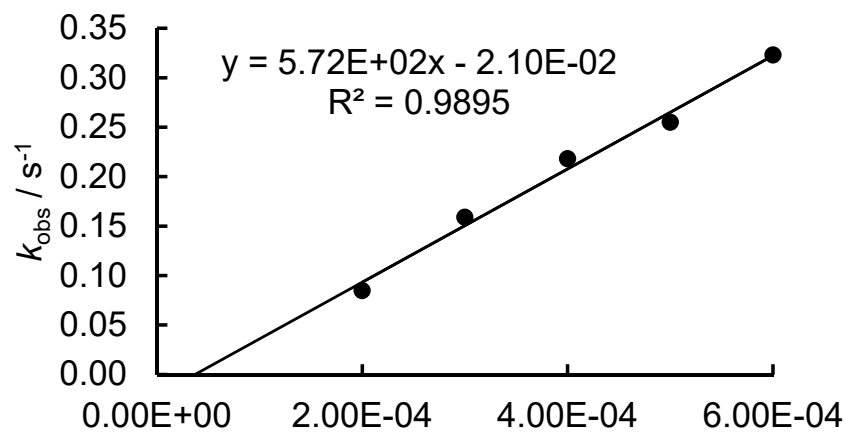

[2a] / M

$\mathbf{1 a}+\mathbf{2} \mathbf{b}$ in DMSO (Stopped-flow, detection at $432 \mathrm{~nm}$ ) AEM-42

\begin{tabular}{llllll}
\hline$[\mathbf{1 a}]_{0}$, & {$[\mathbf{2 b}-\mathrm{H}]_{0}$,} & {$[\mathrm{KOtBu}]_{0}$,} & {$[18-\mathrm{c}-6]_{0}$,} & {$[\mathbf{2 b}]_{0}$,} & $k_{\text {obs, }}$ \\
$\mathrm{M}$ & $\mathrm{M}$ & $\mathrm{M}$ & $\mathrm{M}$ & $\mathrm{M}$ & $\mathrm{s}^{-1}$ \\
\hline $1.88 \times 10^{-5}$ & $4.00 \times 10^{-4}$ & $2.00 \times 10^{-4}$ & & $2.00 \times 10^{-4}$ & $1.75 \times 10^{-2}$ \\
$1.88 \times 10^{-5}$ & $6.00 \times 10^{-4}$ & $3.00 \times 10^{-4}$ & $3.00 \times 10^{-4}$ & $3.00 \times 10^{-4}$ & $2.54 \times 10^{-2}$ \\
$1.88 \times 10^{-5}$ & $8.00 \times 10^{-4}$ & $4.00 \times 10^{-4}$ & & $4.00 \times 10^{-4}$ & $3.29 \times 10^{-2}$ \\
$1.88 \times 10^{-5}$ & $1.00 \times 10^{-3}$ & $5.00 \times 10^{-4}$ & $5.00 \times 10^{-4}$ & $5.00 \times 10^{-4}$ & $4.22 \times 10^{-2}$ \\
$1.88 \times 10^{-5}$ & $1.20 \times 10^{-3}$ & $6.00 \times 10^{-4}$ & & $6.00 \times 10^{-4}$ & $4.85 \times 10^{-2}$ \\
\hline
\end{tabular}

$$
k_{2}=7.88 \times 10^{1} \mathrm{M}^{-1} \mathrm{~s}^{-1}
$$

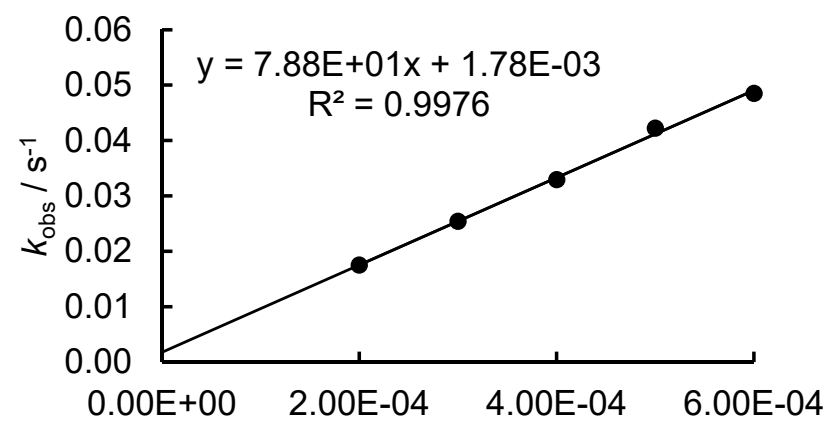

[2b] / M 
$1 a+2 c$ in DMSO (Stopped-flow, detection at $432 \mathrm{~nm}$ ) AEM-43

\begin{tabular}{llllll}
\hline$[\mathbf{1 a}]_{0}$, & {$[\mathbf{2 c - H}]_{0}$,} & {$[\mathrm{KOtBu}]_{0}$,} & {$[18-\mathrm{c}-6]_{0}$,} & {$[\mathbf{2 c}]_{0}$,} & $k_{\text {obs, }}$ \\
$\mathrm{M}$ & $\mathrm{M}$ & $\mathrm{M}$ & $\mathrm{M}$ & $\mathrm{M}$ & $\mathrm{s}^{-1}$ \\
\hline $1.97 \times 10^{-5}$ & $4.00 \times 10^{-4}$ & $2.00 \times 10^{-4}$ & & $2.00 \times 10^{-4}$ & $6.37 \times 10^{-3}$ \\
$1.97 \times 10^{-5}$ & $6.00 \times 10^{-4}$ & $3.00 \times 10^{-4}$ & $3.00 \times 10^{-4}$ & $3.00 \times 10^{-4}$ & $8.62 \times 10^{-3}$ \\
$1.97 \times 10^{-5}$ & $8.00 \times 10^{-4}$ & $4.00 \times 10^{-4}$ & & $4.00 \times 10^{-4}$ & $1.24 \times 10^{-2}$ \\
$1.97 \times 10^{-5}$ & $1.00 \times 10^{-3}$ & $5.00 \times 10^{-4}$ & $5.00 \times 10^{-4}$ & $5.00 \times 10^{-4}$ & $1.55 \times 10^{-2}$ \\
$1.97 \times 10^{-5}$ & $1.20 \times 10^{-3}$ & $6.00 \times 10^{-4}$ & & $6.00 \times 10^{-4}$ & $1.84 \times 10^{-2}$ \\
\hline
\end{tabular}

$$
k_{2}=3.09 \times 10^{1} \mathrm{M}^{-1} \mathrm{~s}^{-1}
$$

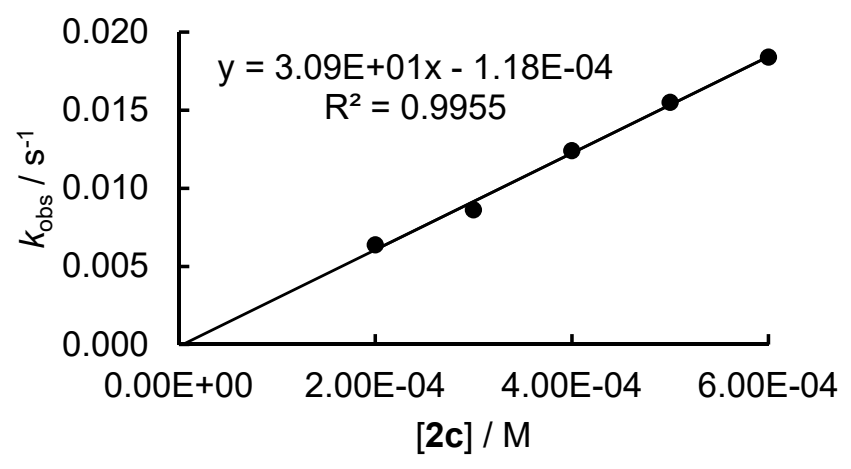

$\mathbf{1 a}+\mathbf{2 d}$ in DMSO (Stopped-flow, detection at $432 \mathrm{~nm}$ ) AEM-44

\begin{tabular}{llllll}
\hline$[\mathbf{1 a}]_{0}$, & {$[\mathbf{2 d - H}]_{0}$,} & {$[\text { KOtBu }]_{0}$,} & {$[18-\mathrm{c}-6]_{0}$,} & {$[\mathbf{2 d}]_{0}$,} & $k_{\text {obs, }}$ \\
$\mathrm{M}$ & $\mathrm{M}$ & $\mathrm{M}$ & $\mathrm{M}$ & $\mathrm{M}$ & $\mathrm{s}^{-1}$ \\
\hline $1.82 \times 10^{-5}$ & $4.00 \times 10^{-4}$ & $2.00 \times 10^{-4}$ & & $2.00 \times 10^{-4}$ & $1.97 \times 10^{-3}$ \\
$1.82 \times 10^{-5}$ & $6.00 \times 10^{-4}$ & $3.00 \times 10^{-4}$ & $3.00 \times 10^{-4}$ & $3.00 \times 10^{-4}$ & $3.20 \times 10^{-3}$ \\
$1.82 \times 10^{-5}$ & $8.00 \times 10^{-4}$ & $4.00 \times 10^{-4}$ & & $4.00 \times 10^{-4}$ & $4.17 \times 10^{-3}$ \\
$1.82 \times 10^{-5}$ & $1.00 \times 10^{-3}$ & $5.00 \times 10^{-4}$ & $5.00 \times 10^{-4}$ & $5.00 \times 10^{-4}$ & $5.15 \times 10^{-3}$ \\
$1.82 \times 10^{-5}$ & $1.20 \times 10^{-3}$ & $6.00 \times 10^{-4}$ & & $6.00 \times 10^{-4}$ & $6.31 \times 10^{-3}$ \\
\hline
\end{tabular}

$$
k_{2}=1.06 \times 10^{1} \mathrm{M}^{-1} \mathrm{~s}^{-1}
$$

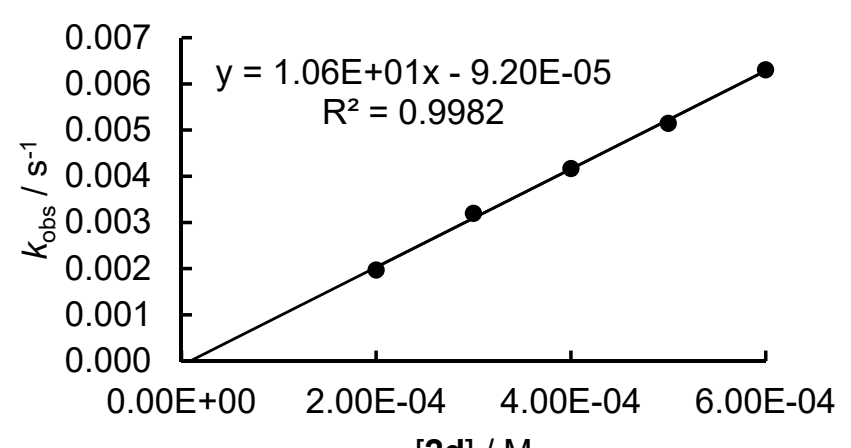

$[2 \mathrm{~d}] / \mathrm{M}$ 
Reactions of $p$ VQM 1b $(\mathrm{R}=\mathrm{H})$ with Reference Nucleophiles

$\mathbf{1 b}+\mathbf{2 a}$ in DMSO (Stopped-flow, detection at $405 \mathrm{~nm}$ ) AEM-26

\begin{tabular}{llllll}
\hline$[\mathbf{1 b}]_{0}$, & {$[\mathbf{2 a}-\mathrm{H}]_{0}$,} & {$[\mathrm{KOtBu}]_{0}$,} & {$[18-\mathrm{c}-6]_{0}$,} & {$[\mathbf{2 a}]_{0}$,} & $k_{\text {obs, }}$ \\
$\mathrm{M}$ & $\mathrm{M}$ & $\mathrm{M}$ & $\mathrm{M}$ & $\mathrm{M}$ & $\mathrm{s}^{-1}$ \\
\hline $1.95 \times 10^{-5}$ & $4.00 \times 10^{-4}$ & $2.00 \times 10^{-4}$ & & $2.00 \times 10^{-4}$ & $1.77 \times 10^{-1}$ \\
$1.95 \times 10^{-5}$ & $6.00 \times 10^{-4}$ & $3.00 \times 10^{-4}$ & $3.00 \times 10^{-4}$ & $3.00 \times 10^{-4}$ & $2.37 \times 10^{-1}$ \\
$1.95 \times 10^{-5}$ & $8.00 \times 10^{-4}$ & $4.00 \times 10^{-4}$ & & $4.00 \times 10^{-4}$ & $3.31 \times 10^{-1}$ \\
$1.95 \times 10^{-5}$ & $1.00 \times 10^{-3}$ & $5.00 \times 10^{-4}$ & $5.00 \times 10^{-4}$ & $5.00 \times 10^{-4}$ & $4.14 \times 10^{-1}$ \\
$1.95 \times 10^{-5}$ & $1.20 \times 10^{-3}$ & $6.00 \times 10^{-4}$ & & $6.00 \times 10^{-4}$ & $4.44 \times 10^{-1}$ \\
\hline
\end{tabular}

$$
k_{2}=7.11 \times 10^{2} \mathrm{M}^{-1} \mathrm{~s}^{-1}
$$

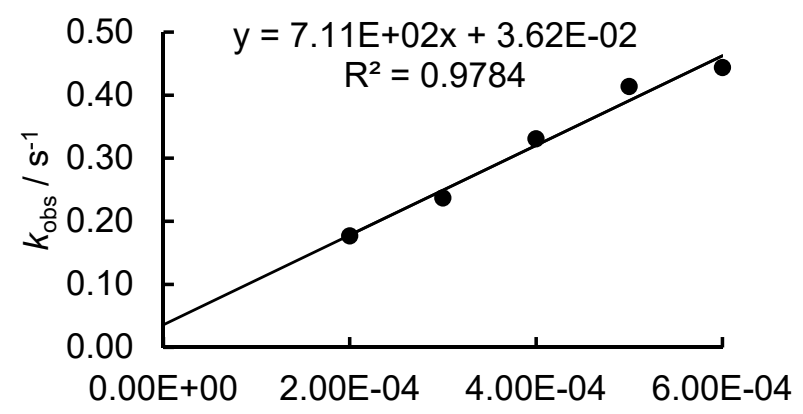

[2a] / M

$\mathbf{1 b}+\mathbf{2} \mathbf{b}$ in DMSO (Stopped-flow, detection at $405 \mathrm{~nm}$ ) AEM-12

\begin{tabular}{llllll}
\hline$[\mathbf{1 b}]_{0}$, & {$[\mathbf{2 b}-\mathrm{H}]_{0}$,} & {$[\mathrm{KOtBu}]_{0}$,} & {$[18-\mathrm{c}-6]_{0}$,} & {$[\mathbf{2 b}]_{0}$,} & $k_{\text {obs, }}$ \\
$\mathrm{M}$ & $\mathrm{M}$ & $\mathrm{M}$ & $\mathrm{M}$ & $\mathrm{M}$ & $\mathrm{s}^{-1}$ \\
\hline $2.00 \times 10^{-5}$ & $1.00 \times 10^{-4}$ & $5.00 \times 10^{-5}$ & & $5.00 \times 10^{-5}$ & $8.45 \times 10^{-3}$ \\
$2.00 \times 10^{-5}$ & $2.00 \times 10^{-4}$ & $1.00 \times 10^{-4}$ & $3.00 \times 10^{-4}$ & $1.00 \times 10^{-4}$ & $1.64 \times 10^{-2}$ \\
$2.00 \times 10^{-5}$ & $3.00 \times 10^{-4}$ & $1.50 \times 10^{-4}$ & & $1.50 \times 10^{-4}$ & $2.54 \times 10^{-2}$ \\
$2.00 \times 10^{-5}$ & $4.00 \times 10^{-3}$ & $2.00 \times 10^{-4}$ & $5.00 \times 10^{-4}$ & $2.00 \times 10^{-4}$ & $3.40 \times 10^{-2}$ \\
$2.00 \times 10^{-5}$ & $5.00 \times 10^{-3}$ & $2.50 \times 10^{-4}$ & & $2.50 \times 10^{-4}$ & $4.10 \times 10^{-2}$ \\
\hline
\end{tabular}

$$
k_{2}=1.65 \times 10^{2} \mathrm{M}^{-1} \mathrm{~s}^{-1}
$$

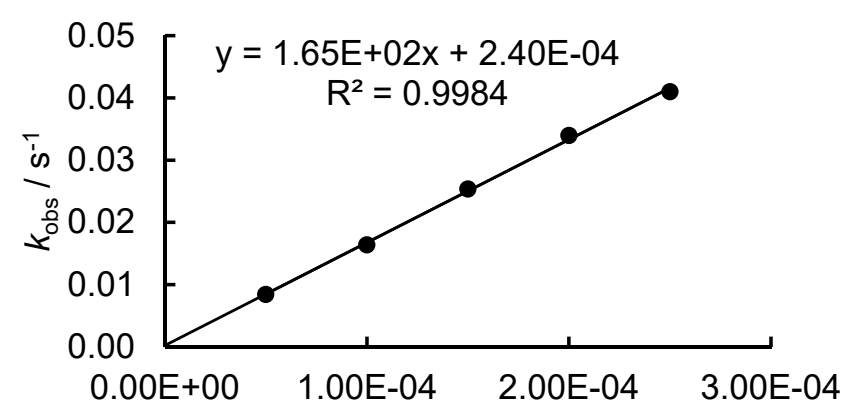

[2b] / M 
$\mathbf{1 b}+\mathbf{2 c}$ in DMSO (Stopped-flow, detection at $405 \mathrm{~nm}$ ) AEM-20

\begin{tabular}{llllll}
\hline$[\mathbf{1 b}]_{0}$, & {$[\mathbf{2 c - H}]_{0}$,} & {$[\mathrm{KOtBu}]_{0}$,} & {$[18-\mathrm{c}-6]_{0}$,} & {$[\mathbf{2 c}]_{0}$,} & $k_{\text {obs, }}$ \\
$\mathrm{M}$ & $\mathrm{M}$ & $\mathrm{M}$ & $\mathrm{M}$ & $\mathrm{M}$ & $\mathrm{s}^{-1}$ \\
\hline $1.49 \times 10^{-5}$ & $4.00 \times 10^{-4}$ & $2.00 \times 10^{-4}$ & & $2.00 \times 10^{-4}$ & $1.19 \times 10^{-2}$ \\
$1.49 \times 10^{-5}$ & $6.00 \times 10^{-4}$ & $3.00 \times 10^{-4}$ & $3.00 \times 10^{-4}$ & $3.00 \times 10^{-4}$ & $1.74 \times 10^{-2}$ \\
$1.49 \times 10^{-5}$ & $8.00 \times 10^{-4}$ & $4.00 \times 10^{-4}$ & & $4.00 \times 10^{-4}$ & $2.39 \times 10^{-2}$ \\
$1.49 \times 10^{-5}$ & $1.00 \times 10^{-3}$ & $5.00 \times 10^{-4}$ & $5.00 \times 10^{-4}$ & $5.00 \times 10^{-4}$ & $2.88 \times 10^{-2}$ \\
$1.49 \times 10^{-5}$ & $1.20 \times 10^{-3}$ & $6.00 \times 10^{-4}$ & & $6.00 \times 10^{-4}$ & $3.70 \times 10^{-2}$ \\
\hline
\end{tabular}

$$
k_{2}=6.16 \times 10^{1} \mathrm{M}^{-1} \mathrm{~s}^{-1}
$$

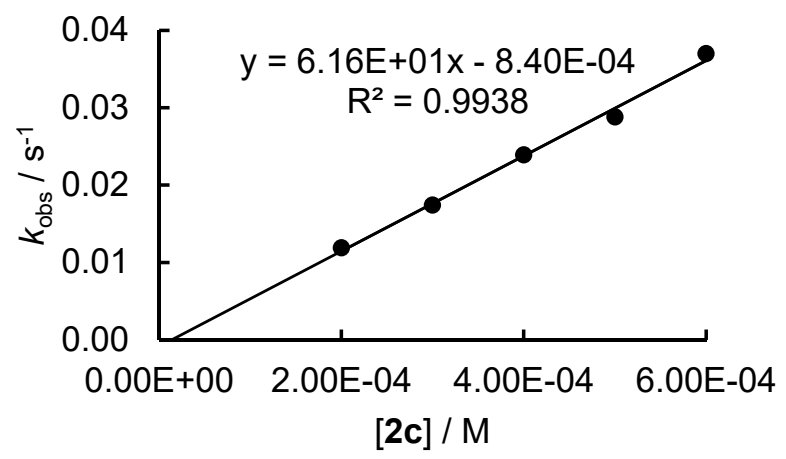

$\mathbf{1 b}+\mathbf{2 d}$ in DMSO (Stopped-flow, detection at $405 \mathrm{~nm}$ ) AEM-19

\begin{tabular}{llllll}
\hline$[\mathbf{1 b}]_{0}$, & {$[\mathbf{2 d}-\mathrm{H}]_{0}$,} & {$[\text { KOtBu }]_{0}$,} & {$[18-\mathrm{c}-6]_{0}$,} & {$[\mathbf{2 d}]_{0}$,} & $k_{\text {obs, }}$ \\
$\mathrm{M}$ & $\mathrm{M}$ & $\mathrm{M}$ & $\mathrm{M}$ & $\mathrm{M}$ & $\mathrm{s}^{-1}$ \\
\hline $1.58 \times 10^{-5}$ & $4.00 \times 10^{-4}$ & $2.00 \times 10^{-4}$ & & $2.00 \times 10^{-4}$ & $4.83 \times 10^{-3}$ \\
$1.58 \times 10^{-5}$ & $6.00 \times 10^{-4}$ & $3.00 \times 10^{-4}$ & $3.00 \times 10^{-4}$ & $3.00 \times 10^{-4}$ & $7.62 \times 10^{-3}$ \\
$1.58 \times 10^{-5}$ & $8.00 \times 10^{-4}$ & $4.00 \times 10^{-4}$ & & $4.00 \times 10^{-4}$ & $1.00 \times 10^{-2}$ \\
$1.58 \times 10^{-5}$ & $1.00 \times 10^{-3}$ & $5.00 \times 10^{-4}$ & $5.00 \times 10^{-4}$ & $5.00 \times 10^{-4}$ & $1.20 \times 10^{-2}$ \\
\hline
\end{tabular}

$$
k_{2}=2.39 \times 10^{1} \mathrm{M}^{-1} \mathrm{~s}^{-1}
$$

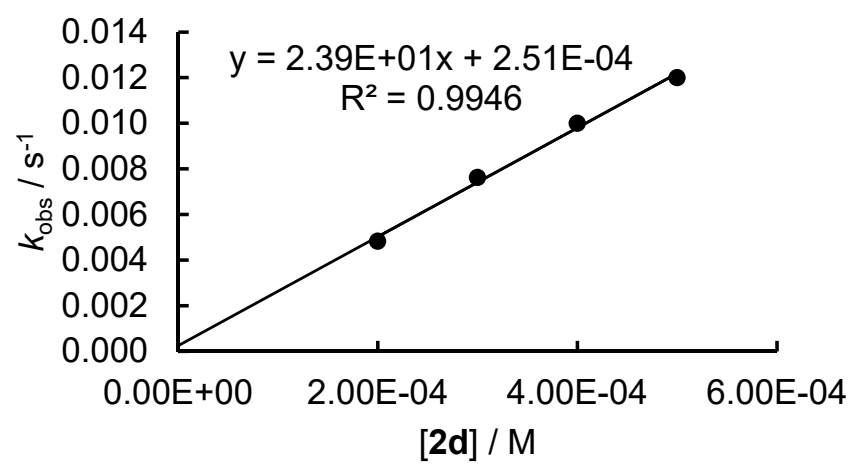




\section{Reactions of $p$ VQM 1c $(\mathrm{R}=\mathrm{Cl})$ with Reference Nucleophiles}

1c $+2 a$ in DMSO (Stopped-flow, detection at $408 \mathrm{~nm}$ ) AEM-46

\begin{tabular}{llllll}
\hline$[\mathbf{1 c}]_{0}$, & {$[\mathbf{2 a}-\mathrm{H}]_{0}$,} & {$[\mathrm{KOtBu}]_{0}$,} & {$[18-\mathrm{c}-6]_{0}$,} & {$[2 \mathrm{a}]_{0}$,} & $k_{\text {obs, }}$ \\
$\mathrm{M}$ & $\mathrm{M}$ & $\mathrm{M}$ & $\mathrm{M}$ & $\mathrm{M}$ & $\mathrm{s}^{-1}$ \\
\hline $1.73 \times 10^{-5}$ & $4.00 \times 10^{-4}$ & $2.00 \times 10^{-4}$ & & $2.00 \times 10^{-4}$ & $2.66 \times 10^{-1}$ \\
$1.73 \times 10^{-5}$ & $6.00 \times 10^{-4}$ & $3.00 \times 10^{-4}$ & $3.00 \times 10^{-4}$ & $3.00 \times 10^{-4}$ & $3.89 \times 10^{-1}$ \\
$1.73 \times 10^{-5}$ & $8.00 \times 10^{-4}$ & $4.00 \times 10^{-4}$ & & $4.00 \times 10^{-4}$ & $5.29 \times 10^{-1}$ \\
$1.73 \times 10^{-5}$ & $1.00 \times 10^{-3}$ & $5.00 \times 10^{-4}$ & $5.00 \times 10^{-4}$ & $5.00 \times 10^{-4}$ & $6.23 \times 10^{-1}$ \\
$1.73 \times 10^{-5}$ & $1.20 \times 10^{-3}$ & $6.00 \times 10^{-4}$ & & $6.00 \times 10^{-4}$ & $7.17 \times 10^{-1}$ \\
\hline
\end{tabular}

$$
k_{2}=1.14 \times 10^{3} \mathrm{M}^{-1} \mathrm{~s}^{-1}
$$

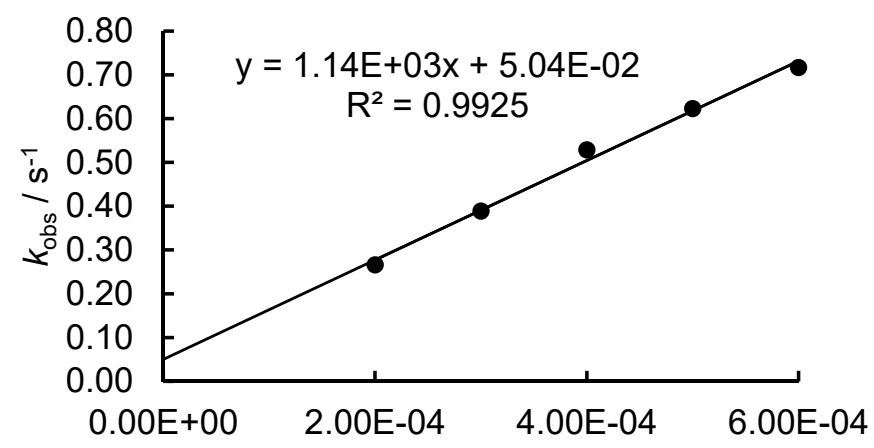

[2a] / M

$\mathbf{1 c}+\mathbf{2 b}$ in DMSO (Stopped-flow, detection at $408 \mathrm{~nm}$ ) AEM-42

\begin{tabular}{llllll}
\hline$[\mathbf{1 c}]_{0}$, & {$[\mathbf{2 b}-\mathrm{H}]_{0}$,} & {$[\mathrm{KOtBu}]_{0}$,} & {$[18-\mathrm{c}-6]_{0}$,} & {$[\mathbf{2 b}]_{0}$,} & $k_{\text {obs, }}$ \\
$\mathrm{M}$ & $\mathrm{M}$ & $\mathrm{M}$ & $\mathrm{M}$ & $\mathrm{M}$ & $\mathrm{s}^{-1}$ \\
\hline $1.75 \times 10^{-5}$ & $4.00 \times 10^{-4}$ & $2.00 \times 10^{-4}$ & & $2.00 \times 10^{-4}$ & $4.52 \times 10^{-2}$ \\
$1.75 \times 10^{-5}$ & $6.00 \times 10^{-4}$ & $3.00 \times 10^{-4}$ & $3.00 \times 10^{-4}$ & $3.00 \times 10^{-4}$ & $6.78 \times 10^{-2}$ \\
$1.75 \times 10^{-5}$ & $8.00 \times 10^{-4}$ & $4.00 \times 10^{-4}$ & & $4.00 \times 10^{-4}$ & $8.82 \times 10^{-2}$ \\
$1.75 \times 10^{-5}$ & $1.00 \times 10^{-3}$ & $5.00 \times 10^{-4}$ & $5.00 \times 10^{-4}$ & $5.00 \times 10^{-4}$ & $1.14 \times 10^{-1}$ \\
$1.75 \times 10^{-5}$ & $1.20 \times 10^{-3}$ & $6.00 \times 10^{-4}$ & & $6.00 \times 10^{-4}$ & $1.36 \times 10^{-1}$ \\
\hline
\end{tabular}

$$
k_{2}=2.28 \times 10^{2} \mathrm{M}^{-1} \mathrm{~s}^{-1}
$$

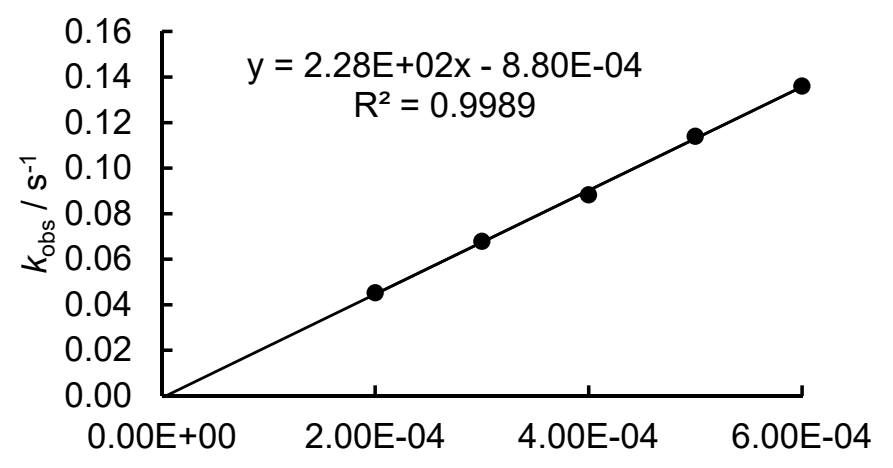

[2b] / M 
$1 c+2 c$ in DMSO (Stopped-flow, detection at $408 \mathrm{~nm}$ ) AEM-43

\begin{tabular}{llllll}
\hline$[\mathbf{1 c}]_{0}$, & {$[\mathbf{2 c - H}]_{0}$,} & {$[\mathrm{KOtBu}]_{0}$,} & {$[18-\mathrm{c}-6]_{0}$,} & {$[\mathbf{2 c}]_{0}$,} & $k_{\text {obs, }}$ \\
$\mathrm{M}$ & $\mathrm{M}$ & $\mathrm{M}$ & $\mathrm{M}$ & $\mathrm{M}$ & $\mathrm{s}^{-1}$ \\
\hline $1.80 \times 10^{-5}$ & $4.00 \times 10^{-4}$ & $2.00 \times 10^{-4}$ & & $2.00 \times 10^{-4}$ & $1.46 \times 10^{-2}$ \\
$1.80 \times 10^{-5}$ & $6.00 \times 10^{-4}$ & $3.00 \times 10^{-4}$ & $3.00 \times 10^{-4}$ & $3.00 \times 10^{-4}$ & $2.00 \times 10^{-2}$ \\
$1.80 \times 10^{-5}$ & $8.00 \times 10^{-4}$ & $4.00 \times 10^{-4}$ & & $4.00 \times 10^{-4}$ & $2.94 \times 10^{-2}$ \\
$1.80 \times 10^{-5}$ & $1.00 \times 10^{-3}$ & $5.00 \times 10^{-4}$ & $5.00 \times 10^{-4}$ & $5.00 \times 10^{-4}$ & $3.68 \times 10^{-2}$ \\
$1.80 \times 10^{-5}$ & $1.20 \times 10^{-3}$ & $6.00 \times 10^{-4}$ & & $6.00 \times 10^{-4}$ & $4.34 \times 10^{-2}$ \\
\hline
\end{tabular}

$$
k_{2}=7.44 \times 10^{1} \mathrm{M}^{-1} \mathrm{~s}^{-1}
$$

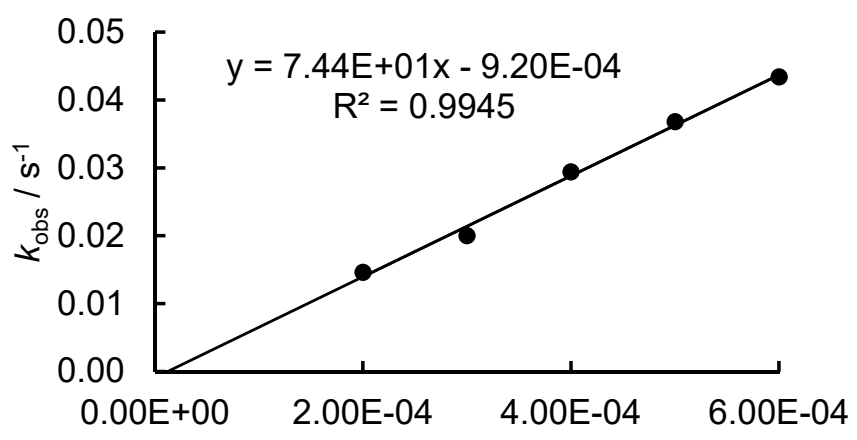

[2c] / M

1c $+\mathbf{2 d}$ in DMSO (Stopped-flow, detection at $408 \mathrm{~nm}$ ) AEM-44

\begin{tabular}{llllll}
\hline$[\mathbf{1 c}]_{0}$, & {$[\mathbf{2 d - H}]_{0}$,} & {$[\mathrm{KOtBu}]_{0}$,} & {$[18-\mathrm{c}-6]_{0}$,} & {$[\mathbf{2 d}]_{0}$,} & $k_{\text {obs, }}$ \\
$\mathrm{M}$ & $\mathrm{M}$ & $\mathrm{M}$ & $\mathrm{M}$ & $\mathrm{M}$ & $\mathrm{s}^{-1}$ \\
\hline $1.65 \times 10^{-5}$ & $4.00 \times 10^{-4}$ & $2.00 \times 10^{-4}$ & & $2.00 \times 10^{-4}$ & $4.04 \times 10^{-3}$ \\
$1.65 \times 10^{-5}$ & $6.00 \times 10^{-4}$ & $3.00 \times 10^{-4}$ & $3.00 \times 10^{-4}$ & $3.00 \times 10^{-4}$ & $6.82 \times 10^{-3}$ \\
$1.65 \times 10^{-5}$ & $8.00 \times 10^{-4}$ & $4.00 \times 10^{-4}$ & & $4.00 \times 10^{-4}$ & $9.14 \times 10^{-3}$ \\
$1.65 \times 10^{-5}$ & $1.00 \times 10^{-3}$ & $5.00 \times 10^{-4}$ & $5.00 \times 10^{-4}$ & $5.00 \times 10^{-4}$ & $1.14 \times 10^{-2}$ \\
$1.65 \times 10^{-5}$ & $1.20 \times 10^{-3}$ & $6.00 \times 10^{-4}$ & & $6.00 \times 10^{-4}$ & $1.41 \times 10^{-2}$ \\
\hline
\end{tabular}

$$
k_{2}=2.47 \times 10^{1} \mathrm{M}^{-1} \mathrm{~s}^{-1}
$$

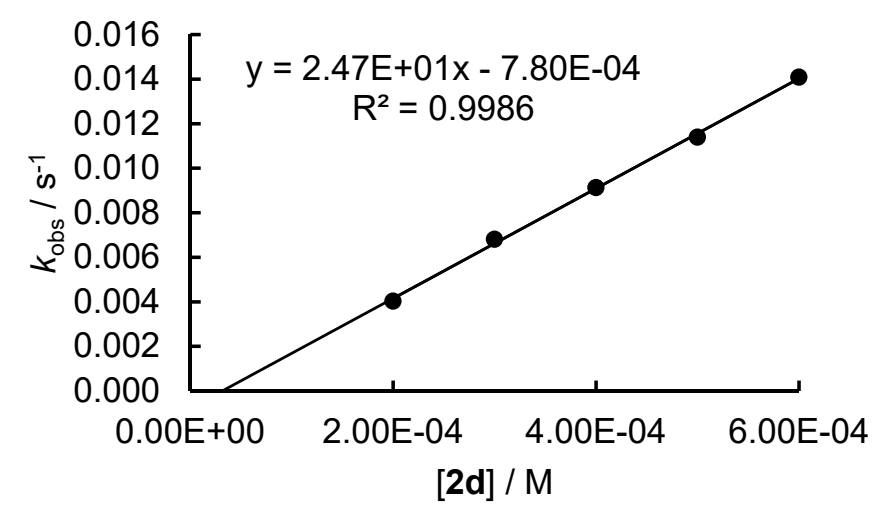




\section{Reactions of $p V Q M 1 d\left(R=\mathrm{NO}_{2}\right)$ with Reference Nucleophiles}

1d + 2a in DMSO (Stopped-flow, detection at $429 \mathrm{~nm}$ ) AEM-47

\begin{tabular}{llllll}
\hline$[\mathbf{1 d}]_{0}$, & {$[\mathbf{2 a}-\mathrm{H}]_{0}$,} & {$[\mathrm{KOtBu}]_{0}$,} & {$[18-\mathrm{c}-6]_{0}$,} & {$[\mathbf{2 a}]_{0}$,} & $k_{\text {obs, }}$ \\
$\mathrm{M}$ & $\mathrm{M}$ & $\mathrm{M}$ & $\mathrm{M}$ & $\mathrm{M}$ & $\mathrm{s}^{-1}$ \\
\hline $1.91 \times 10^{-5}$ & $4.00 \times 10^{-4}$ & $2.00 \times 10^{-4}$ & & $2.00 \times 10^{-4}$ & $6.29 \times 10^{-1}$ \\
$1.91 \times 10^{-5}$ & $6.00 \times 10^{-4}$ & $3.00 \times 10^{-4}$ & $3.00 \times 10^{-4}$ & $3.00 \times 10^{-4}$ & $9.20 \times 10^{-1}$ \\
$1.91 \times 10^{-5}$ & $8.00 \times 10^{-4}$ & $4.00 \times 10^{-4}$ & & $4.00 \times 10^{-4}$ & 1.28 \\
$1.91 \times 10^{-5}$ & $1.00 \times 10^{-3}$ & $5.00 \times 10^{-4}$ & $5.00 \times 10^{-4}$ & $5.00 \times 10^{-4}$ & 1.76 \\
$1.91 \times 10^{-5}$ & $1.20 \times 10^{-3}$ & $6.00 \times 10^{-4}$ & & $6.00 \times 10^{-4}$ & 1.93 \\
\hline
\end{tabular}

$$
k_{2}=3.44 \times 10^{3} \mathrm{M}^{-1} \mathrm{~s}^{-1}
$$

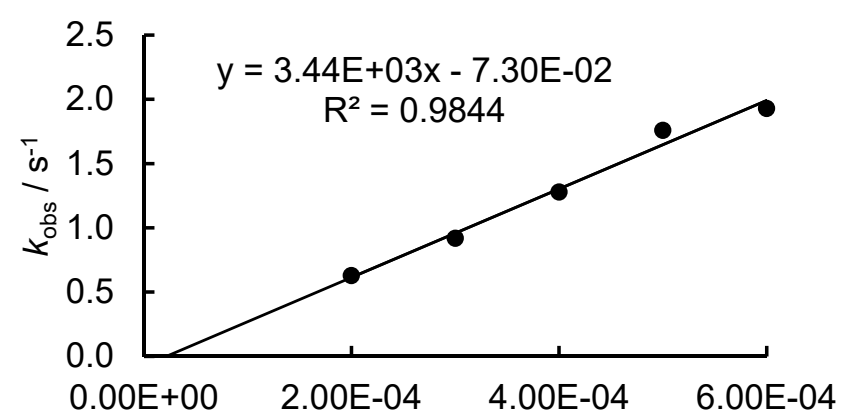

[2a] / M

$\mathbf{1 d}+\mathbf{2 b}$ in DMSO (Stopped-flow, detection at $429 \mathrm{~nm}$ ) AEM-42

\begin{tabular}{llllll}
\hline$[\mathbf{1 d}]_{0}$, & {$[\mathbf{2 b}-\mathrm{H}]_{0}$,} & {$[\mathrm{KOtBu}]_{0}$,} & {$[18-\mathrm{c}-6]_{0}$,} & {$[\mathbf{2 b}]_{0}$,} & $k_{\text {obs, }}$ \\
$\mathrm{M}$ & $\mathrm{M}$ & & $\mathrm{M}$ & $\mathrm{M}$ & $\mathrm{s}^{-1}$ \\
\hline $1.85 \times 10^{-5}$ & $4.00 \times 10^{-4}$ & $2.00 \times 10^{-4}$ & & $2.00 \times 10^{-4}$ & $1.17 \times 10^{-1}$ \\
$1.85 \times 10^{-5}$ & $6.00 \times 10^{-4}$ & $3.00 \times 10^{-4}$ & $3.00 \times 10^{-4}$ & $3.00 \times 10^{-4}$ & $1.78 \times 10^{-1}$ \\
$1.85 \times 10^{-5}$ & $8.00 \times 10^{-4}$ & $4.00 \times 10^{-4}$ & & $4.00 \times 10^{-4}$ & $2.32 \times 10^{-1}$ \\
$1.85 \times 10^{-5}$ & $1.00 \times 10^{-3}$ & $5.00 \times 10^{-4}$ & $5.00 \times 10^{-4}$ & $5.00 \times 10^{-4}$ & $2.96 \times 10^{-1}$ \\
$1.85 \times 10^{-5}$ & $1.20 \times 10^{-3}$ & $6.00 \times 10^{-4}$ & & $6.00 \times 10^{-4}$ & $3.54 \times 10^{-1}$ \\
\hline
\end{tabular}

$$
k_{2}=5.92 \times 10^{2} \mathrm{M}^{-1} \mathrm{~s}^{-1}
$$

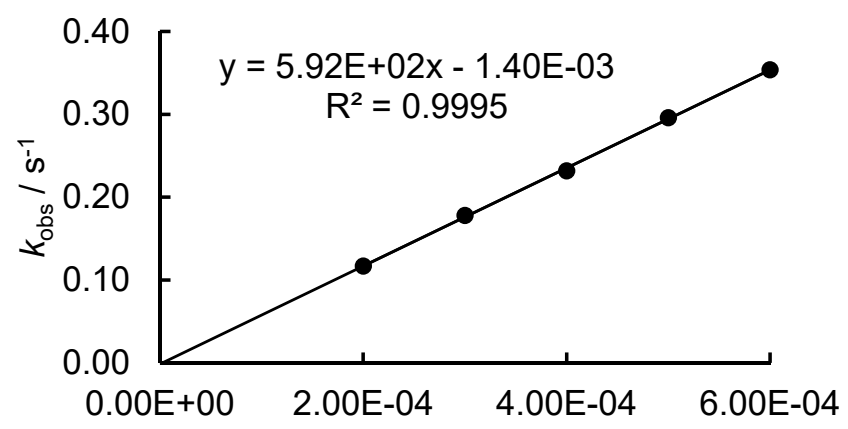

[2b] / M 
$\mathbf{1 d}+\mathbf{2 c}$ in DMSO (Stopped-flow, detection at $429 \mathrm{~nm}$ ) AEM-43

\begin{tabular}{llllll}
\hline$[\mathbf{1 d}]_{0}$, & {$[\mathbf{2 c - H}]_{0}$,} & {$[\mathrm{KOtBu}]_{0}$,} & {$[18-\mathrm{c}-6]_{0}$,} & {$[\mathbf{2 c}]_{0}$,} & $k_{\text {obs, }}$ \\
$\mathrm{M}$ & $\mathrm{M}$ & $\mathrm{M}$ & $\mathrm{M}$ & $\mathrm{M}$ & $\mathrm{s}^{-1}$ \\
\hline $1.81 \times 10^{-5}$ & $4.00 \times 10^{-4}$ & $2.00 \times 10^{-4}$ & & $2.00 \times 10^{-4}$ & $3.33 \times 10^{-2}$ \\
$1.81 \times 10^{-5}$ & $6.00 \times 10^{-4}$ & $3.00 \times 10^{-4}$ & $3.00 \times 10^{-4}$ & $3.00 \times 10^{-4}$ & $4.62 \times 10^{-2}$ \\
$1.81 \times 10^{-5}$ & $8.00 \times 10^{-4}$ & $4.00 \times 10^{-4}$ & & $4.00 \times 10^{-4}$ & $6.69 \times 10^{-2}$ \\
$1.81 \times 10^{-5}$ & $1.00 \times 10^{-3}$ & $5.00 \times 10^{-4}$ & $5.00 \times 10^{-4}$ & $5.00 \times 10^{-4}$ & $8.32 \times 10^{-2}$ \\
$1.81 \times 10^{-5}$ & $1.20 \times 10^{-3}$ & $6.00 \times 10^{-4}$ & & $6.00 \times 10^{-4}$ & $9.80 \times 10^{-2}$ \\
\hline
\end{tabular}

$$
k_{2}=1.66 \times 10^{2} \mathrm{M}^{-1} \mathrm{~s}^{-1}
$$

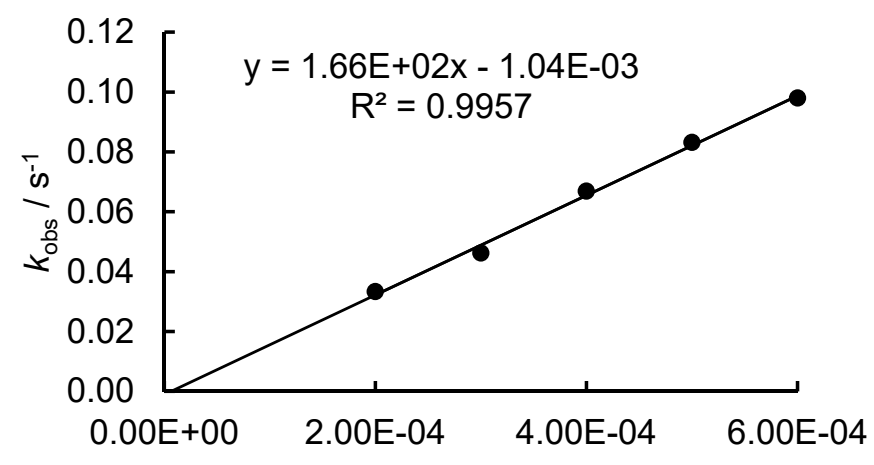

$[2 \mathrm{c}] / \mathrm{M}$

$\mathbf{1 d}+\mathbf{2 d}$ in DMSO (Stopped-flow, detection at $429 \mathrm{~nm}$ ) AEM-44

\begin{tabular}{llllll}
\hline$[\mathbf{1 d}]_{0}$, & {$[\mathbf{2 d}-\mathrm{H}]_{0}$,} & {$[\mathrm{KOtBu}]_{0}$,} & {$[18-\mathrm{c}-6]_{0}$,} & {$[\mathbf{2 d}]_{0}$,} & $k_{\text {obs, }}$ \\
$\mathrm{M}$ & $\mathrm{M}$ & $\mathrm{M}$ & $\mathrm{M}$ & $\mathrm{M}$ & $\mathrm{s}^{-1}$ \\
\hline $1.89 \times 10^{-5}$ & $4.00 \times 10^{-4}$ & $2.00 \times 10^{-4}$ & & $2.00 \times 10^{-4}$ & $8.20 \times 10^{-3}$ \\
$1.89 \times 10^{-5}$ & $6.00 \times 10^{-4}$ & $3.00 \times 10^{-4}$ & $3.00 \times 10^{-4}$ & $3.00 \times 10^{-4}$ & $1.38 \times 10^{-3}$ \\
$1.89 \times 10^{-5}$ & $8.00 \times 10^{-4}$ & $4.00 \times 10^{-4}$ & & $4.00 \times 10^{-4}$ & $1.84 \times 10^{-2}$ \\
$1.89 \times 10^{-5}$ & $1.00 \times 10^{-3}$ & $5.00 \times 10^{-4}$ & $5.00 \times 10^{-4}$ & $5.00 \times 10^{-4}$ & $2.32 \times 10^{-2}$ \\
$1.89 \times 10^{-5}$ & $1.20 \times 10^{-3}$ & $6.00 \times 10^{-4}$ & & $6.00 \times 10^{-4}$ & $2.89 \times 10^{-2}$ \\
\hline
\end{tabular}

$$
k_{2}=5.08 \times 10^{1} \mathrm{M}^{-1} \mathrm{~s}^{-1}
$$

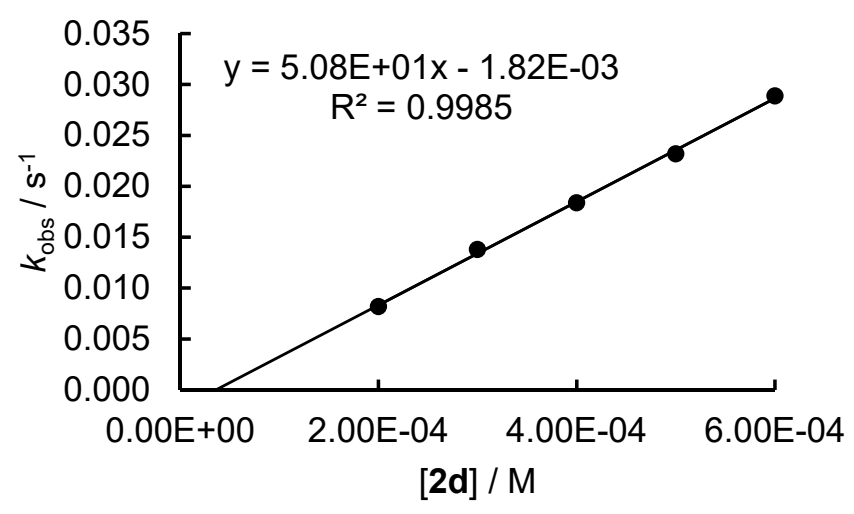




\section{Computational Details}

Initially, all systems were subjected to a conformational search with the OPLS3 ${ }^{58}$ force field as implemented in MacroModel ${ }^{59}$ with the MCMM method in gas phase. Next, all conformers were preoptimized with the semiempirical $\mathrm{RM}^{\mathrm{S} 10}$ method as included in the NDDO package in Maestro. Redundant conformers were eliminated and the 10 energetically lowest lying species optimized at the M06-2X/6-31 $+g(d, p)^{S 11}$ level of theory considering solvation by the SMD model for DMSO. ${ }^{S 12}$ Thermal corrections at $298 \mathrm{~K}$ were obtained at the same level of theory from vibrational frequencies and are unscaled. Transition states were validated by the presence of one imaginary frequency and IRC calculations. A free energy correction $+7.91 \mathrm{~kJ} \mathrm{~mol}^{-1}$ was applied to all free energies to consider the conversion from gas phase (1 atm) to liquid phase $(1 \mathrm{M})$. All quantum-chemical calculations were performed with the Gaussian 16 A.03 set of codes. ${ }^{\text {S13 }}$

Electronic and Gibbs energies for all species at the SMD(DMSO)/M06-2X/6-31+G(d,p) level.

\begin{tabular}{llll} 
1b & & & \\
File & $E_{\text {tot }}$ & $G_{298}$ & Contribution \\
\hline REDO_3_1 & -968.150050 & -967.674054 & \\
\hline
\end{tabular}

\begin{tabular}{llll}
$\begin{array}{l}\text { 2d } \\
\text { File }\end{array}$ & $\mathrm{E}_{\text {tot }}$ & $\mathrm{G}_{298}$ & Contribution \\
\hline $\mathrm{mn} \_1$ & -224.442965 & -224.405465 & \\
\hline
\end{tabular}

\begin{tabular}{llll}
$\mathbf{2 b}$ & & & \\
File & $E_{\text {tot }}$ & $G_{298}$ & Contribution \\
\hline em_1 & -574.199477 & -574.061010 & 0.271863 \\
em_2 & -574.199092 & -574.061940 & 0.728137 \\
Boltzmann weighted & & -574.061687 & \\
\hline
\end{tabular}


Reaction of $\mathbf{1 b}+\mathbf{2} b$ to give $\mathbf{3 b b}$ :

\begin{tabular}{llll}
$\begin{array}{l}\text { TS }(\mathbf{1} b+\mathbf{2} b \text { to } 3 \mathbf{b b}) \\
\text { File }\end{array}$ & $\mathrm{E}_{\text {tot }}$ & $\mathrm{G}_{298}$ & Contribution \\
\hline REDO_hqm_em_16_1_ts1 & -1542.342416 & -1541.782078 & 0.000028 \\
REDO_hqm_em_16_3_ts1 & -1542.345085 & -1541.783450 & 0.000121 \\
REDO_hqm_em_16_7_ts1 & -1542.353519 & -1541.791958 & 0.999825 \\
REDO_hqm_em_16_8_ts1 & -1542.341930 & -1541.781992 & 0.000026 \\
Boltzmann weighted & & -1541.791956 & \\
\hline
\end{tabular}

\begin{tabular}{|c|c|c|c|}
\hline $\begin{array}{l}3 \mathbf{b b}^{-} \text {anion } \\
\text { File0 }\end{array}$ & $E_{\text {tot }}$ & $\mathrm{G}_{298}$ & Contribution \\
\hline REDO_hqm_em_16_1 & -1542.392798 & -1541.828614 & 0.068262 \\
\hline REDO_hqm_em_16_2 & -1542.392662 & -1541.827399 & 0.018828 \\
\hline REDO_hqm_em_16_3 & -1542.393374 & -1541.828198 & 0.043891 \\
\hline REDO_hqm_em_16_4 & -1542.392678 & -1541.828533 & 0.062615 \\
\hline REDO_hqm_em_16_5 & -1542.392400 & -1541.829094 & 0.113514 \\
\hline REDO_hqm_em_16_6 & -1542.391572 & -1541.828385 & 0.053508 \\
\hline REDO_hqm_em_16_7 & -1542.392678 & -1541.828532 & 0.062561 \\
\hline REDO_hqm_em_16_8 & -1542.392140 & -1541.829603 & 0.194722 \\
\hline REDO_hqm_em_16_9 & -1542.392132 & -1541.827685 & 0.025497 \\
\hline REDO_hqm_em_16_10 & -1542.392655 & -1541.830174 & 0.356602 \\
\hline Boltzmann weighted & & -1541.829330 & \\
\hline
\end{tabular}

\begin{tabular}{llll} 
3bb & & & \\
File & $\mathrm{E}_{\text {tot }}$ & $\mathrm{G}_{298}$ & Contribution \\
\hline REDO_hqm_em_16_pr_1 & -1542.378233 & -1541.815935 & 0.059448 \\
REDO_hqm_em_16_pr_2 & -1542.378673 & -1541.816445 & 0.102169 \\
REDO_hqm_em_16_pr_3 & -1542.378595 & -1541.817127 & 0.210492 \\
REDO_hqm_em_16_pr_4 & -1542.378674 & -1541.816486 & 0.106627 \\
REDO_hqm_em_16_pr_5 & -1542.378677 & -1541.817081 & 0.200349 \\
REDO_hqm_em_16_pr_6 & -1542.377458 & -1541.814668 & 0.015517 \\
REDO_hqm_em_16_pr_7 & -1542.377594 & -1541.816214 & 0.079967 \\
REDO_hqm_em_16_pr_8 & -1542.377749 & -1541.816494 & 0.107611 \\
REDO_hqm_em_16_pr_9 & -1542.378232 & -1541.815927 & 0.058996 \\
REDO_hqm_em_16_pr_10 & -1542.377710 & -1541.815925 & 0.058824 \\
Boltzmann weighted & & -1541.816588 & \\
\hline
\end{tabular}


Reaction of $\mathbf{1 b}+\mathbf{2 b}$ to give $\mathbf{4 b b}$ :

\begin{tabular}{llll} 
TS $(\mathbf{1} \mathbf{b}+\mathbf{2} \mathbf{b}$ to $\mathbf{4 b b})$ & & & \\
File & Etot $_{\text {tot }}$ & $\mathrm{G}_{298}$ & Contribution \\
\hline REDO_hqm_em_18_4_ts1 & -1542.352674 & -1541.790485 & 0.508813 \\
REDO_hqm_em_18_6_ts1 & -1542.351174 & -1541.790337 & 0.435098 \\
REDO_hqm_em_18_10_ts1 & -1542.349178 & -1541.788405 & 0.056089 \\
Boltzmann weighted & & -1541.790304 & \\
\hline
\end{tabular}

\begin{tabular}{llll} 
4bb-Anion & & & \\
File & $E_{\text {tot }}$ & $G_{298}$ & Contribution \\
\hline REDO_hqm_em_18_1 & -1542.393464 & -1541.831095 & 0.114920 \\
REDO_hqm_em_18_2 & -1542.393464 & -1541.831098 & 0.115282 \\
REDO_hqm_em_18_3 & -1542.395309 & -1541.831239 & 0.133852 \\
REDO_hqm_em_18_4 & -1542.392391 & -1541.829490 & 0.020963 \\
REDO_hqm_em_18_5 & -1542.389145 & -1541.824973 & 0.000174 \\
REDO_hqm_em_18_6 & -1542.394391 & -1541.832518 & 0.519424 \\
REDO_hqm_em_18_7 & -1542.388495 & -1541.825697 & 0.000376 \\
REDO_hqm_em_18_8 & -1542.390592 & -1541.826909 & 0.001358 \\
REDO_hqm_em_18_9 & -1542.392147 & -1541.829169 & 0.014905 \\
REDO_hqm_em_18_10 & -1542.394143 & -1541.830739 & 0.078746 \\
Boltzmann weighted & & -1541.831755 & \\
\hline
\end{tabular}

\begin{tabular}{llll} 
4bb & & & \\
File & $\mathrm{E}_{\text {tot }}$ & $\mathrm{G}_{298}$ & Contribution \\
\hline REDO_hqm_em_18_pr_1 & -1542.379708 & -1541.817145 & 0.132348 \\
REDO_hqm_em_18_pr_2 & -1542.380362 & -1541.817381 & 0.170071 \\
REDO_hqm_em_18_pr_3 & -1542.376815 & -1541.816045 & 0.041251 \\
REDO_hqm_em_18_pr_4 & -1542.379316 & -1541.817447 & 0.182267 \\
REDO_hqm_em_18_pr_5 & -1542.377229 & -1541.815063 & 0.014555 \\
REDO_hqm_em_18_pr_6 & -1542.379627 & -1541.816933 & 0.105776 \\
REDO_hqm_em_18_pr_7 & -1542.379296 & -1541.817192 & 0.139104 \\
REDO_hqm_em_18_pr_8 & -1542.377565 & -1541.816067 & 0.042228 \\
REDO_hqm_em_18_pr_9 & -1542.380320 & -1541.817169 & 0.135870 \\
REDO_hqm_em_18_pr_10 & -1542.377670 & -1541.815931 & 0.036531 \\
Boltzmann weighted & & -1541.817062 & \\
\hline
\end{tabular}


Reaction of $\mathbf{1 b}+\mathbf{2 d}$ to give $\mathbf{3 b d}$ :

\begin{tabular}{llll}
$\begin{array}{l}\text { TS }(\mathbf{1} b+\mathbf{2} \mathbf{d} \text { to } 3 \mathbf{b d}) \\
\text { File }\end{array}$ & $\mathrm{E}_{\text {tot }}$ & $\mathrm{G}_{298}$ & Contribution \\
\hline REDO_hqm_mn_16_1_ts1 & -1192.578195 & -1192.155588 & 0.000623 \\
REDO_hqm_mn_16_5_ts1 & -1192.585589 & -1192.162228 & 0.710752 \\
REDO_hqm_mn_16_10_ts1 & -1192.584962 & -1192.161378 & 0.288625 \\
Boltzmann weighted & & -1192.161978 & \\
\hline
\end{tabular}

\begin{tabular}{llll} 
3bd-Anion & & & \\
File & $E_{\text {tot }}$ & G $_{298}$ & Contribution \\
\hline REDO_hqm_mn_16_1 & -1192.610470 & -1192.183580 & 0.024436 \\
REDO_hqm_mn_16_2 & -1192.609503 & -1192.182883 & 0.011664 \\
REDO_hqm_mn_16_3 & -1192.609393 & -1192.182532 & 0.008040 \\
REDO_hqm_mn_16_4 & -1192.610344 & -1192.183226 & 0.016788 \\
REDO_hqm_mn_16_5 & -1192.611185 & -1192.184510 & 0.065505 \\
REDO_hqm_mn_16_6 & -1192.611130 & -1192.185484 & 0.183833 \\
REDO_hqm_mn_16_7 & -1192.609894 & -1192.183271 & 0.017597 \\
REDO_hqm_mn_16_8 & -1192.609957 & -1192.183162 & 0.015685 \\
REDO_hqm_mn_16_9 & -1192.610857 & -1192.184348 & 0.055118 \\
REDO_hqm_mn_16_10 & -1192.611725 & -1192.186602 & 0.601334 \\
Boltzmann weighted & & -1192.185816 & \\
\hline
\end{tabular}

\begin{tabular}{llll} 
3bd & & & \\
File & $E_{\text {tot }}$ & $\mathrm{G}_{298}$ & Contribution \\
\hline REDO_hqm_mn_16_pr_1 & -1192.623996 & -1192.198780 & 0.164436 \\
REDO_hqm_mn_16_pr_2 & -1192.623903 & -1192.198493 & 0.121291 \\
REDO_hqm_mn_16_pr_5 & -1192.623854 & -1192.197956 & 0.068602 \\
REDO_hqm_mn_16_pr_6 & -1192.623674 & -1192.199441 & 0.331329 \\
REDO_hqm_mn_16_pr_7 & -1192.623923 & -1192.198134 & 0.082847 \\
REDO_hqm_mn_16_pr_8 & -1192.623684 & -1192.198350 & 0.104242 \\
REDO_hqm_mn_16_pr_10 & -1192.623668 & -1192.198538 & 0.127255 \\
Boltzmann weighted & & -1192.198779 & \\
\hline
\end{tabular}


Reaction of $\mathbf{1 b}+\mathbf{2 d}$ to give $\mathbf{4 b d}$ :

\begin{tabular}{llll} 
TS $(\mathbf{1} \mathbf{b}+\mathbf{2} \mathbf{d}$ to $\mathbf{4 b d})$ & & & \\
File & $\mathrm{E}_{\text {tot }}$ & $\mathrm{G}_{298}$ & Contribution \\
\hline REDO_hqm_mn_18_1_ts1 & -1192.582933 & -1192.159366 & 0.456196 \\
REDO_hqm_mn_18_7_ts1 & -1192.583031 & -1192.159532 & 0.543804 \\
Boltzmann weighted & & -1192.159457 & \\
\hline
\end{tabular}

\begin{tabular}{llll} 
4bd-Anion \\
File & $\mathrm{E}_{\text {tot }}$ & $\mathrm{G}_{298}$ & Contribution \\
\hline REDO_hqm_mn_18_1 & -1192.611935 & -1192.184228 & 0.007944 \\
REDO_hqm_mn_18_2 & -1192.612925 & -1192.186152 & 0.061138 \\
REDO_hqm_mn_18_3 & -1192.611434 & -1192.184667 & 0.012656 \\
REDO_hqm_mn_18_4 & -1192.611689 & -1192.184828 & 0.015020 \\
REDO_hqm_mn_18_5 & -1192.613030 & -1192.185756 & 0.040165 \\
REDO_hqm_mn_18_6 & -1192.611675 & -1192.184218 & 0.007860 \\
REDO_hqm_mn_18_7 & -1192.613880 & -1192.188009 & 0.437722 \\
REDO_hqm_mn_18_8 & -1192.611107 & -1192.185006 & 0.018133 \\
REDO_hqm_mn_18_9 & -1192.610238 & -1192.183008 & 0.002179 \\
REDO_hqm_mn_18_10 & -1192.613851 & -1192.187917 & 0.397182 \\
Boltzmann weighted & & -1192.187553 & \\
\hline
\end{tabular}

\begin{tabular}{llll} 
4bd & & & \\
File & $E_{\text {tot }}$ & $G_{298}$ & Contribution \\
\hline REDO_hqm_mn_18_pr_1 & -1192.624046 & -1192.198187 & 0.110377 \\
REDO_hqm_mn_18_pr_3 & -1192.623956 & -1192.198266 & 0.120073 \\
REDO_hqm_mn_18_pr_4 & -1192.623983 & -1192.198663 & 0.182894 \\
REDO_hqm_mn_18_pr_5 & -1192.624038 & -1192.199423 & 0.409448 \\
REDO_hqm_mn_18_pr_6 & -1192.623970 & -1192.197254 & 0.041078 \\
REDO_hqm_mn_18_pr_8 & -1192.623833 & -1192.198385 & 0.136129 \\
Boltzmann weighted & & -1192.198778 & \\
\hline
\end{tabular}




\section{Summary of Quantum-Chemical Calculations}

Summary of calculated Gibbs energies for the reactions of the $p V Q M \mathbf{1} \mathbf{b}$ with the nucleophiles ion $\mathbf{2} \mathbf{b}$ and $\mathbf{2} \mathbf{d}$ at the $S M D(D M S O) / M 06-2 X / 6-31+G(d, p)$ level of theory:

\begin{tabular}{|c|c|c|c|c|}
\hline & & $\mathrm{G}_{298}$ [Hartree] & & {$[\mathrm{kJ} / \mathrm{mol}]$} \\
\hline $1 b$ & REDO_3_1 & -967.674054 & & \\
\hline $2 b$ & weighted & -224.405465 & & \\
\hline $\mathrm{TS}(1 b+2 b$ to $3 b b)$ & weighted & -1541.791956 & $\Delta \mathrm{G}^{\ddagger}=$ & 44.0 \\
\hline 3bb-Anion & weighted & -1541.829330 & $\Delta_{\mathrm{r}} \mathrm{G}^{0}=$ & -54.2 \\
\hline $3 b b$ & weighted & -1541.816588 & $\Delta_{\mathrm{r}} \mathrm{G}^{0}=$ & -20.7 \\
\hline $\mathrm{TS}(1 \mathrm{~b}+\mathbf{2 b}$ to $4 \mathrm{bb})$ & weighted & -1541.790304 & $\Delta \mathrm{G}^{\ddagger}=$ & 48.3 \\
\hline 4bb-Anion & weighted & -1541.831755 & $\Delta_{\mathrm{r}} \mathrm{G}^{0}=$ & -60.5 \\
\hline $4 \mathrm{bb}$ & weighted & -1541.817062 & $\Delta_{\mathrm{r}} \mathrm{G}^{0}=$ & -22.0 \\
\hline $2 d$ & mn_1 & & & \\
\hline TS $(\mathbf{1 b}+\mathbf{2 d}$ to $\mathbf{3 b b})$ & weighted & -1192.161978 & $\Delta \mathrm{G}^{\ddagger}=$ & 61.7 \\
\hline 3bd-Anion & weighted & -1192.185816 & $\Delta_{\mathrm{r}} \mathrm{G}^{0}=$ & -0.9 \\
\hline $3 b d$ & weighted & -1192.198779 & $\Delta_{\mathrm{r}} \mathrm{G}^{0}=$ & -35.0 \\
\hline $\mathrm{TS}(\mathbf{1 b}+\mathbf{2 d}$ to $\mathbf{4 b b})$ & weighted & -1192.159457 & $\Delta \mathrm{G}^{\ddagger}=$ & 68.3 \\
\hline 4bd-Anion & weighted & -1192.187553 & $\Delta_{\mathrm{r}} \mathrm{G}^{0}=$ & -5.5 \\
\hline $4 \mathrm{bd}$ & weighted & -1192.198778 & $\Delta_{\mathrm{r}} \mathrm{G}^{0}=$ & -35.0 \\
\hline
\end{tabular}




\section{Copies of NMR Spectra}

品

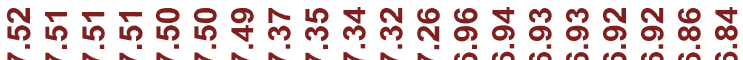

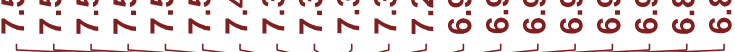

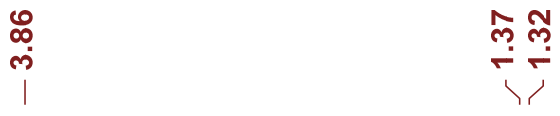

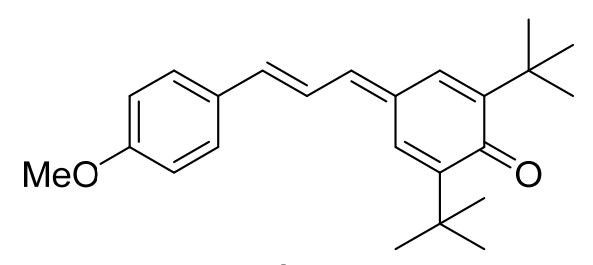

$1 \mathrm{a}$

${ }^{1} \mathrm{H}$ NMR $\left(599 \mathrm{MHz}, \mathrm{CDCl}_{3}\right)$
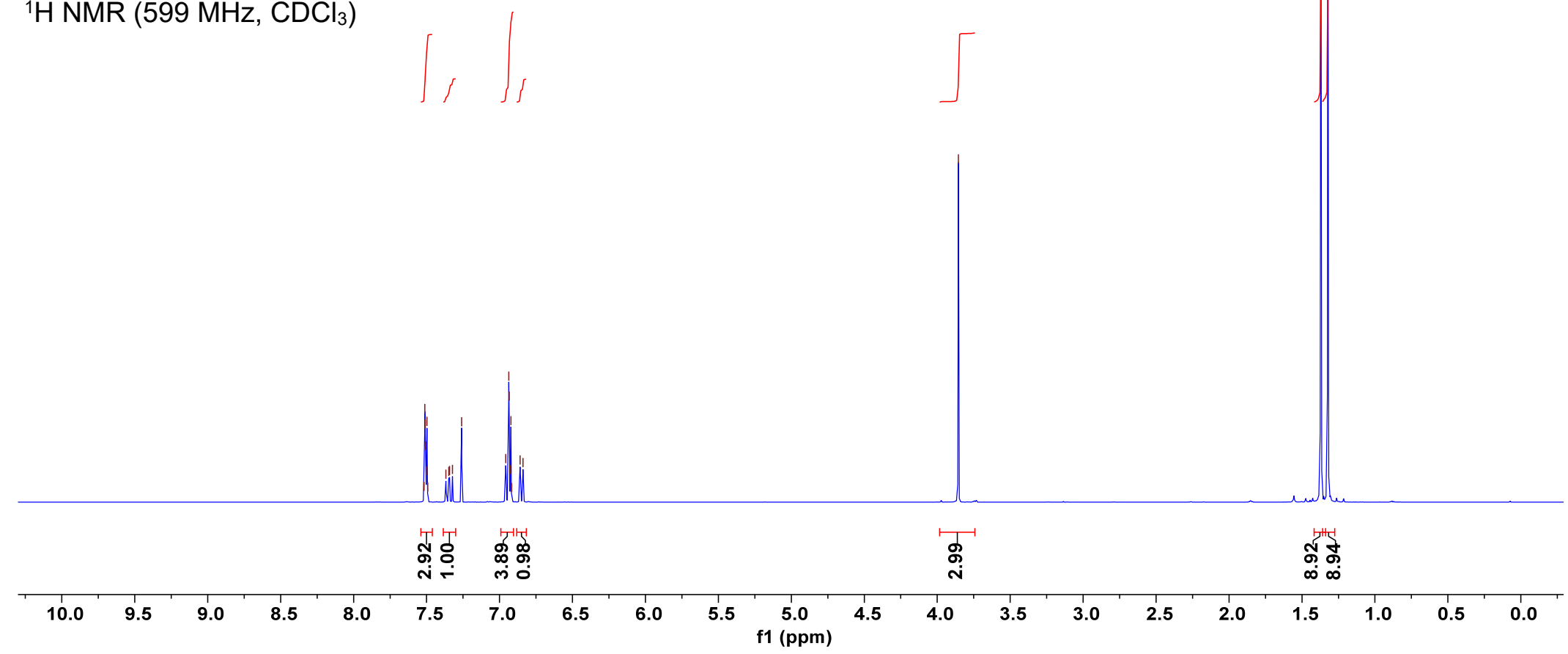


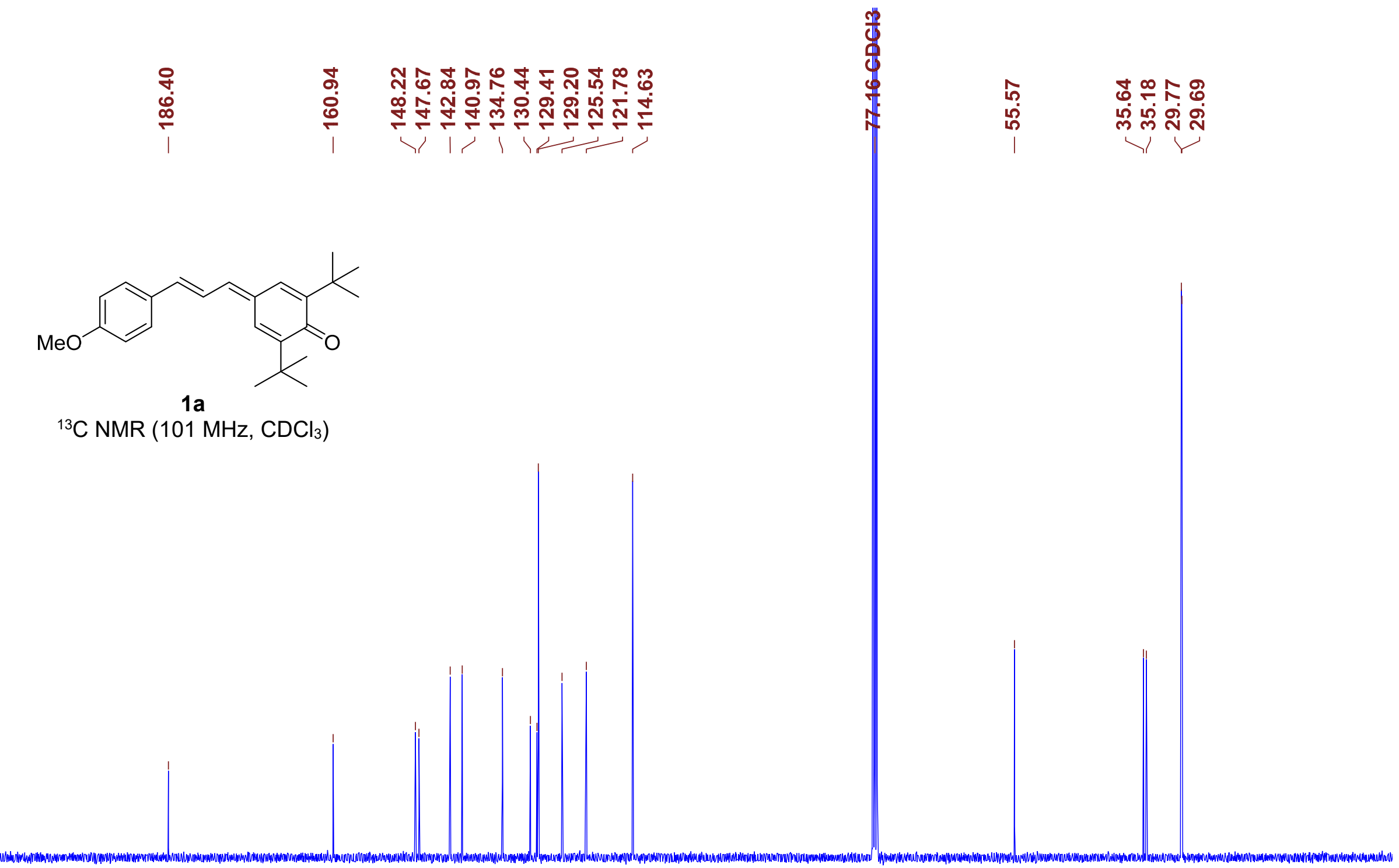

$\begin{array}{llllllll}210 & 200 & 190 & 180 & 170 & 160 & 150 & 140\end{array}$

$\begin{array}{llll}110 \quad 100 & 90 & 80 \\ \mathrm{f} 1(\mathrm{ppm}) & \end{array}$

$80 \quad 70 \quad 60 \quad 50$

40 


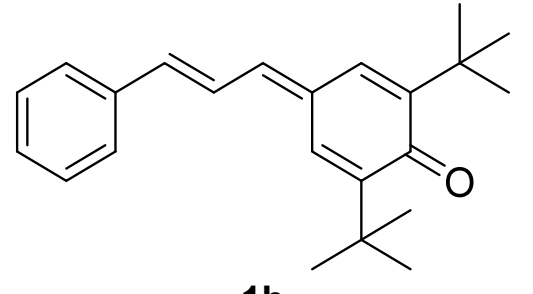

$1 \mathrm{~b}$

${ }^{1} \mathrm{H}$ NMR $\left(400 \mathrm{MHz}, \mathrm{CDCl}_{3}\right)$ $\frac{m}{0}$

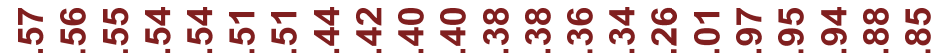

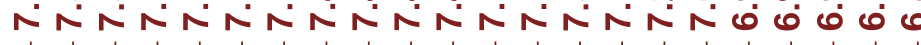

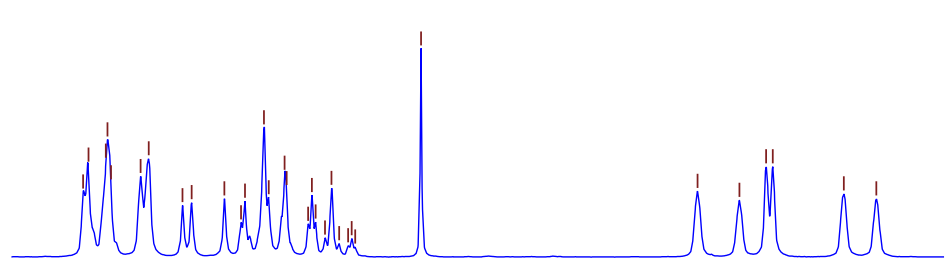

$\begin{array}{llll}7.1 & 7.0 & 6.9 & 6.8\end{array}$ f1 (ppm) 


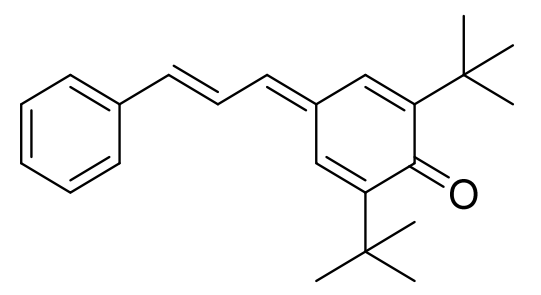

1b

${ }^{13} \mathrm{C}$ NMR $\left(101 \mathrm{MHz}, \mathrm{CDCl}_{3}\right)$
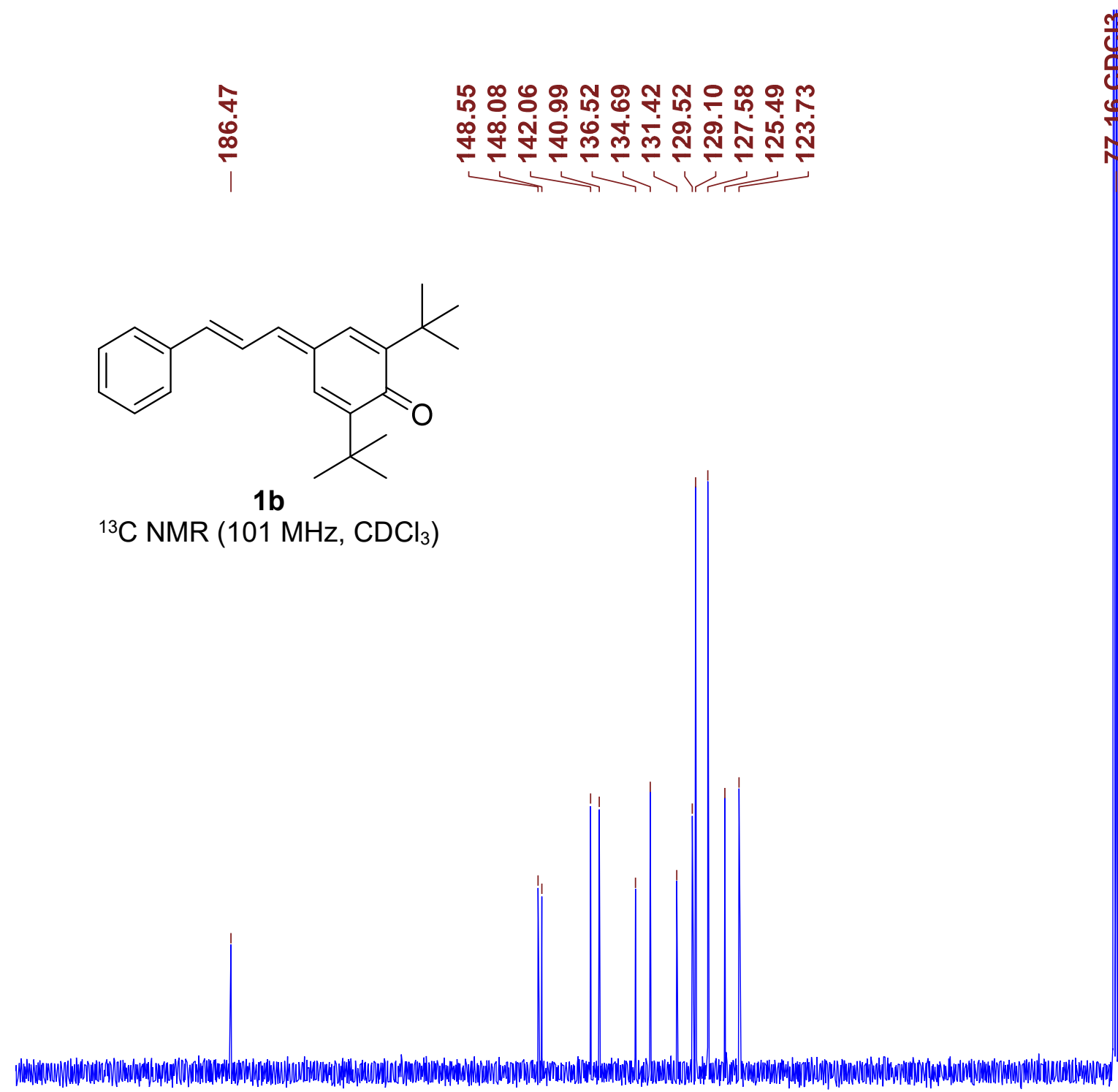


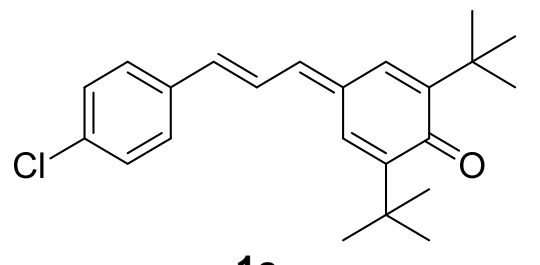

1c

${ }^{1} \mathrm{H}$ NMR (599 MHz, $\mathrm{CDCl}_{3}$ )

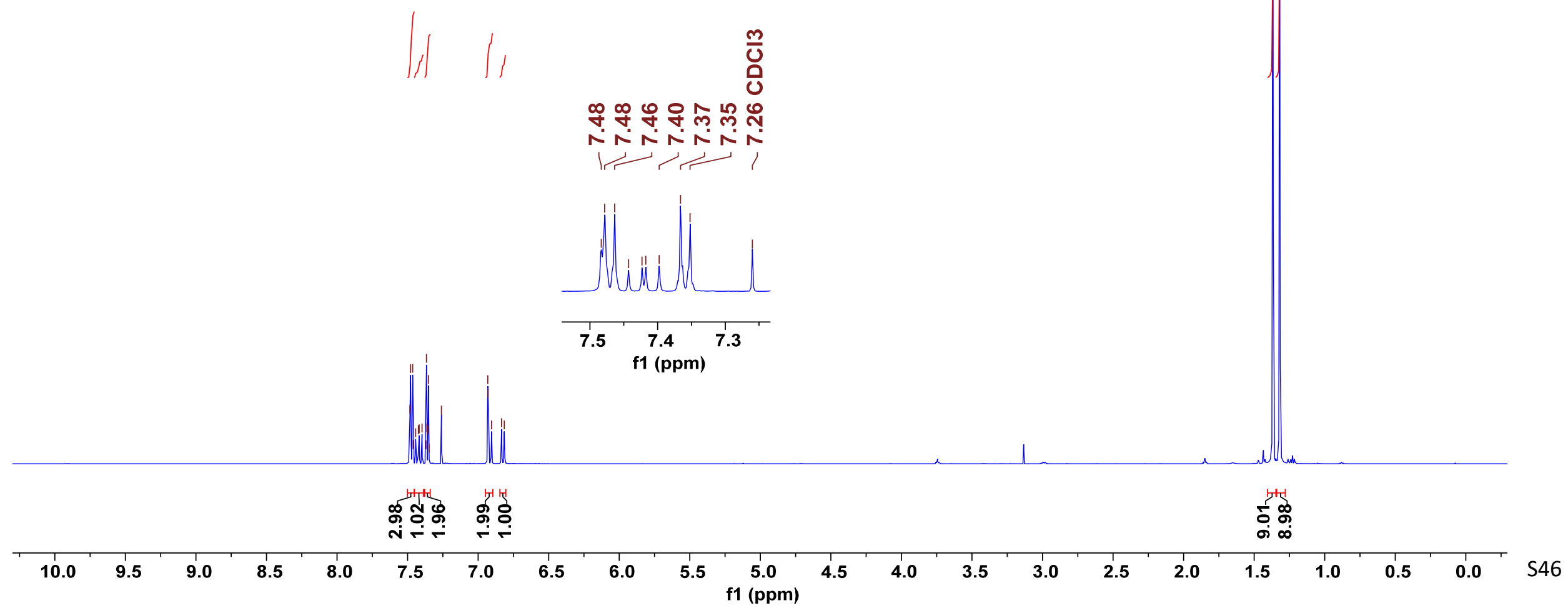




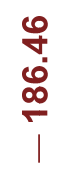

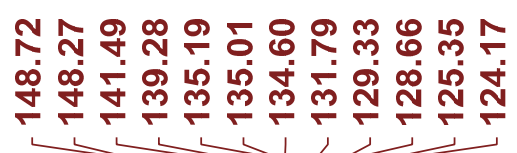

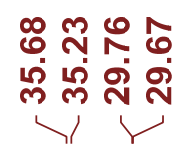

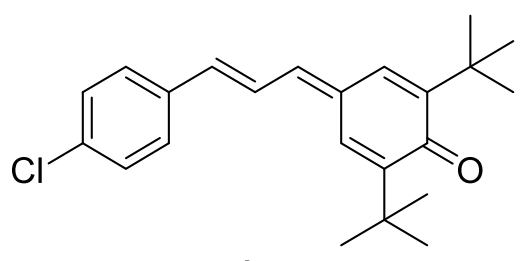

${ }^{13} \mathrm{C} \mathrm{NMR}\left(101 \mathrm{MHz}, \mathrm{CDCl}_{3}\right)$
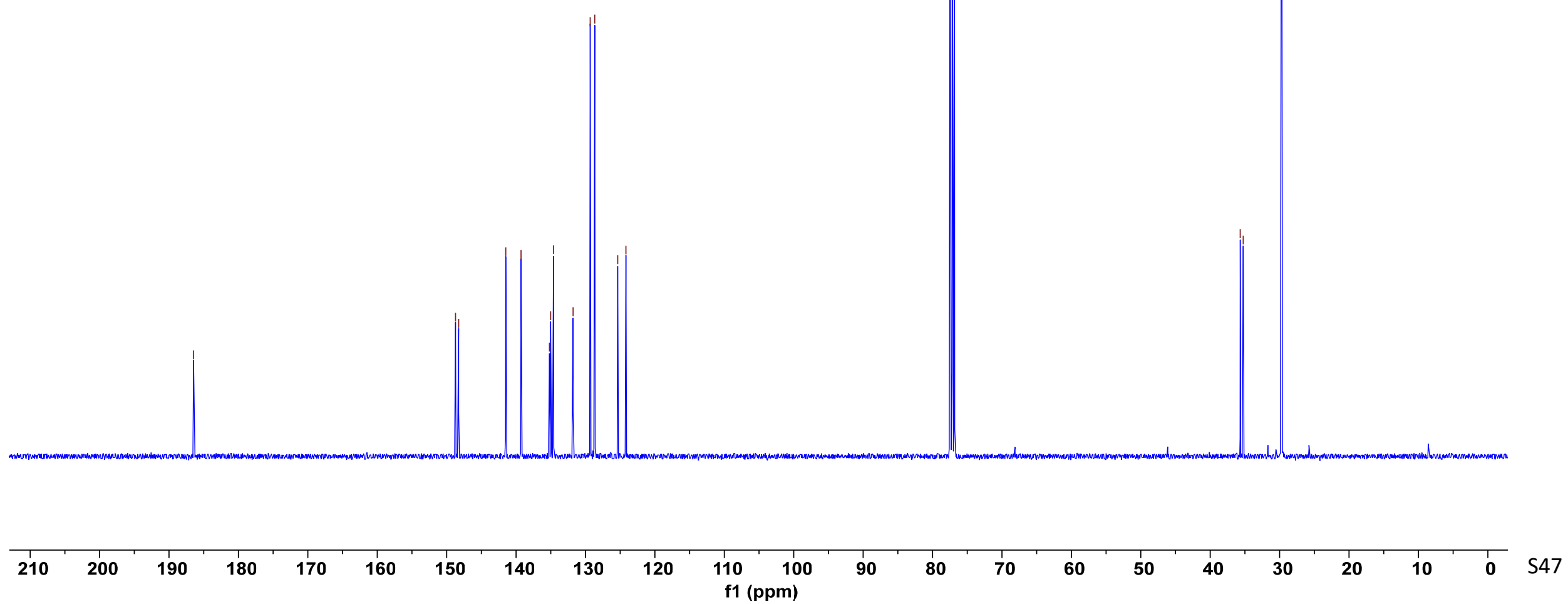


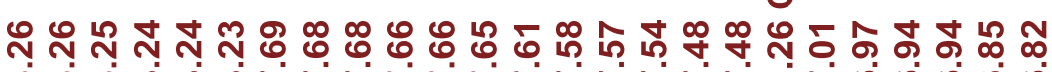

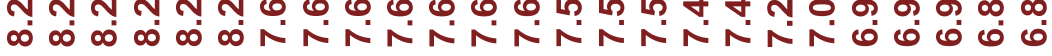

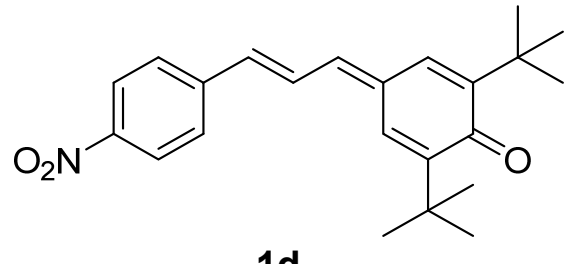

$1 d$

${ }^{1} \mathrm{H}$ NMR $\left(400 \mathrm{MHz}, \mathrm{CDCl}_{3}\right)$

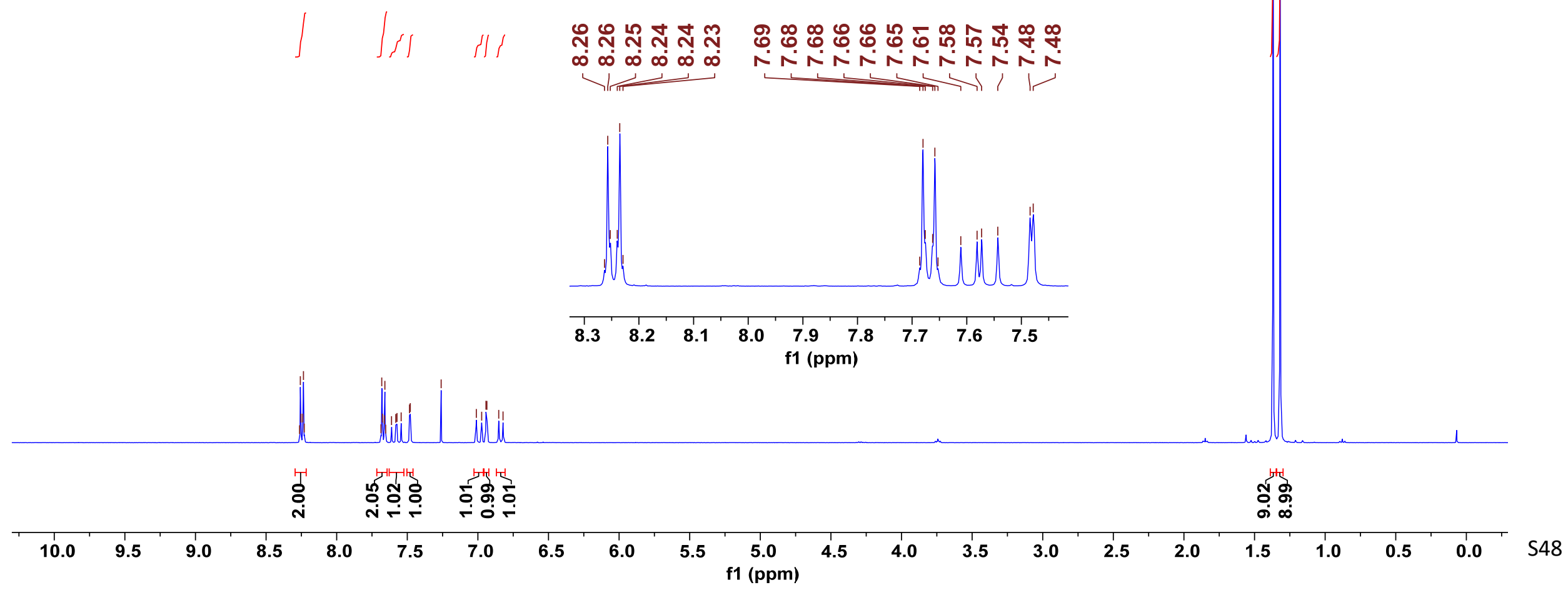




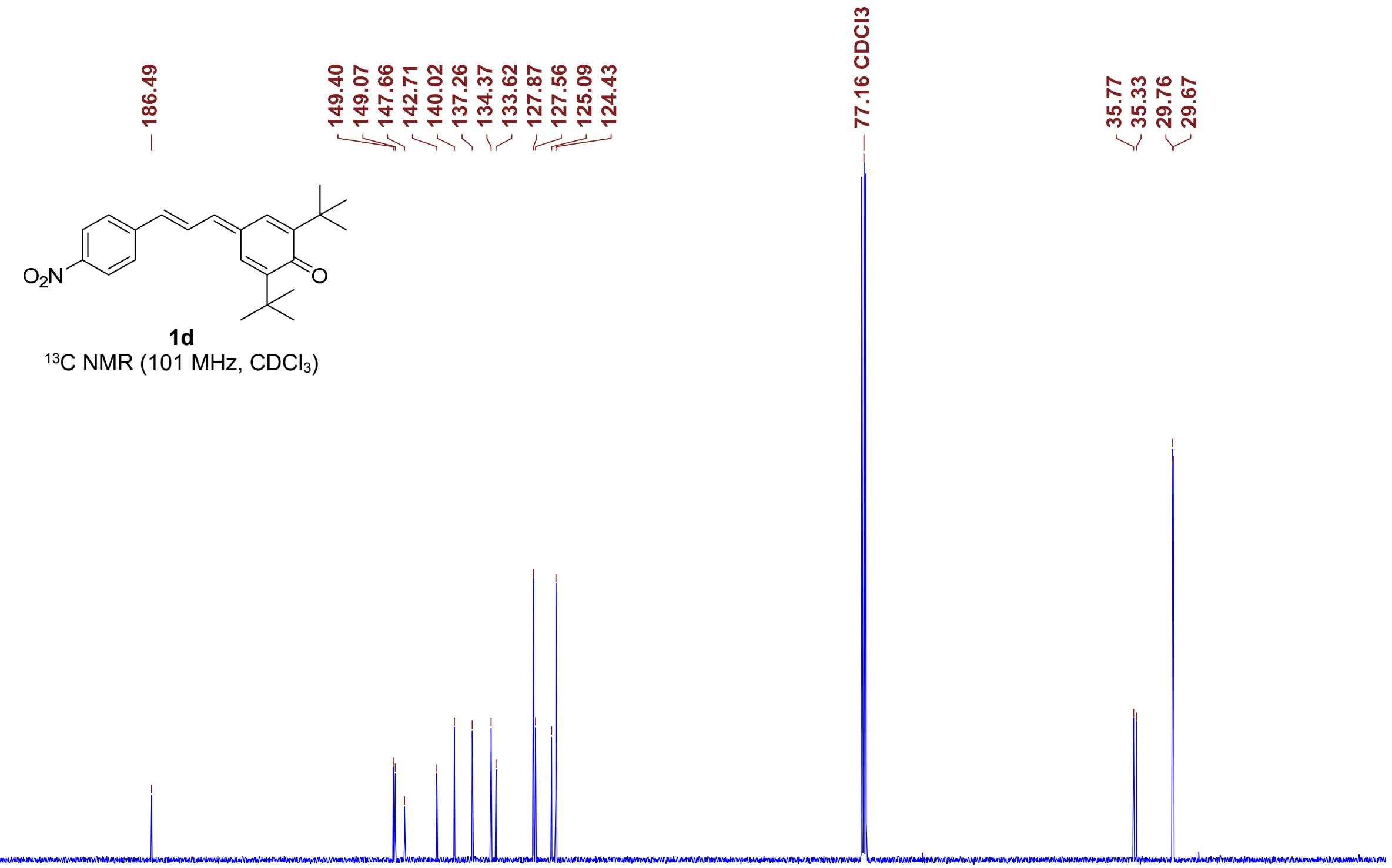

$\begin{array}{lllllll}210 & 200 & 190 & 180 & 170 & 160 & 150\end{array}$ 
誉

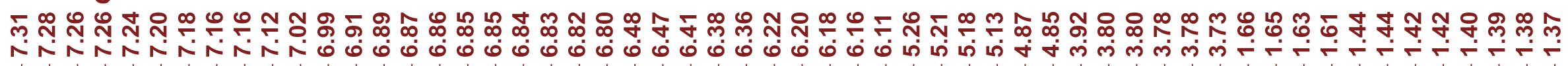
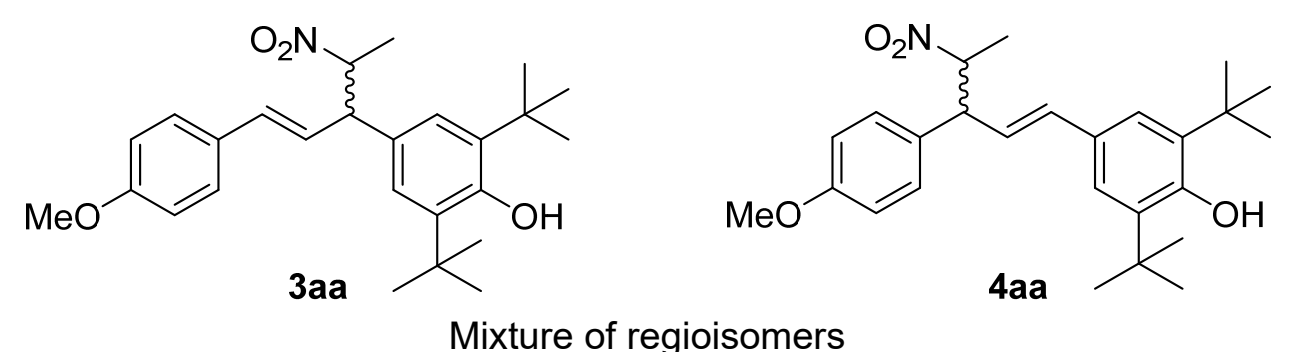

gioisomers

${ }^{1} \mathrm{H} \mathrm{NMR}\left(400 \mathrm{MHz}, \mathrm{CDCl}_{3}\right)$

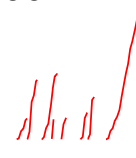




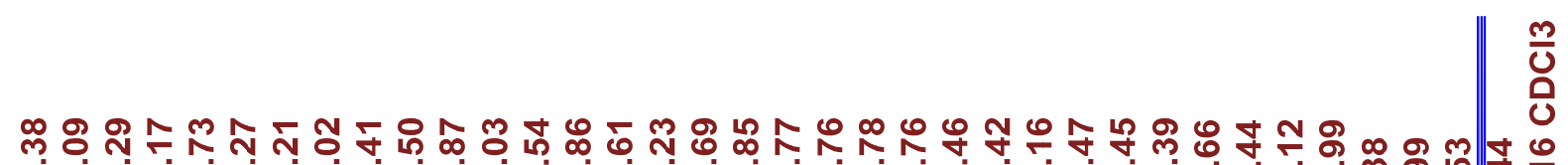

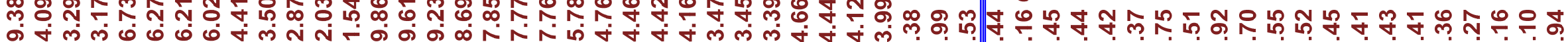

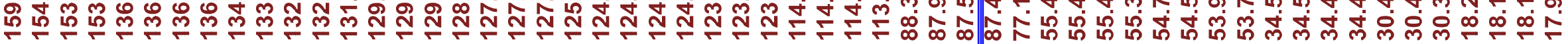<smiles>COc1ccc(/C=C/[C@H](c2cc(C(C)(C)C)c(O)c(C(C)(C)C)c2)C(C)[N+](=O)[O-])cc1</smiles><smiles>COc1ccc(C(/C=C/c2cc(C(C)(C)C)c(O)c(C(C)(C)C)c2)C(C)[N+](=O)[O-])cc1</smiles>

Mixture of regioisomers

${ }^{13} \mathrm{C}$ NMR $\left(101 \mathrm{MHz}, \mathrm{CDCl}_{3}\right)$
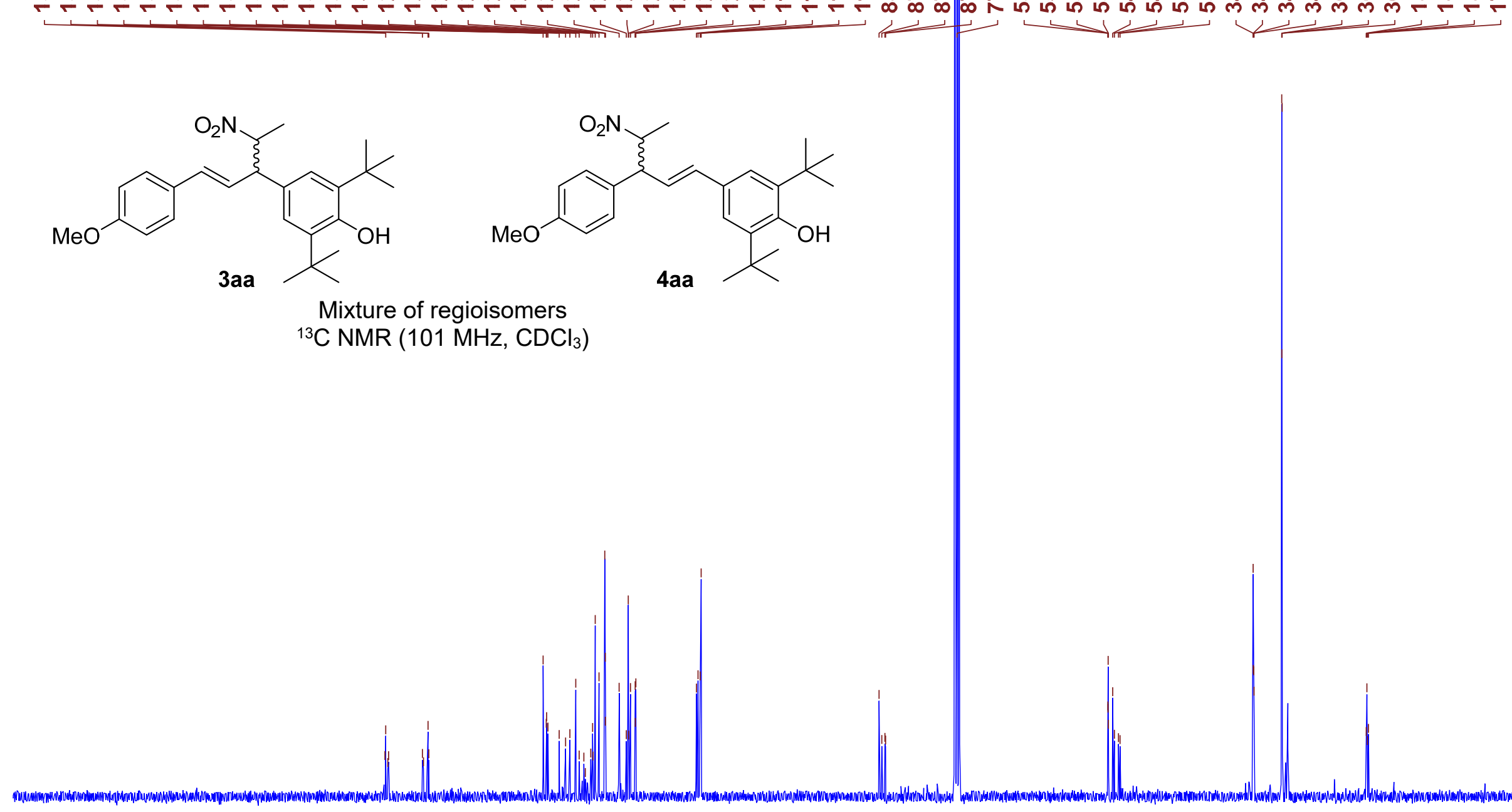
鱼

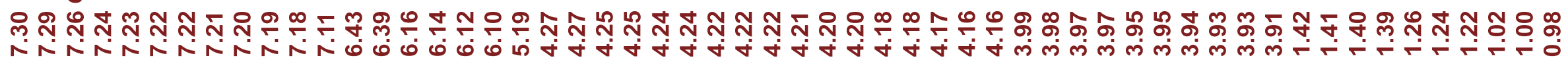

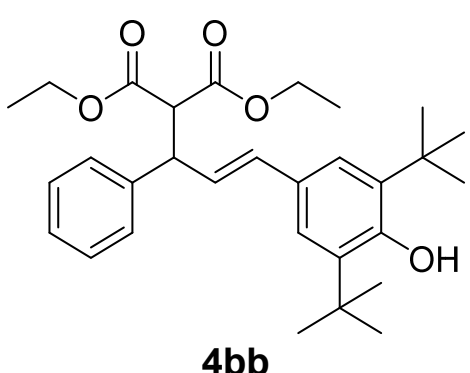

${ }^{1} \mathrm{H} \mathrm{NMR}\left(400 \mathrm{MHz}, \mathrm{CDCl}_{3}\right)$

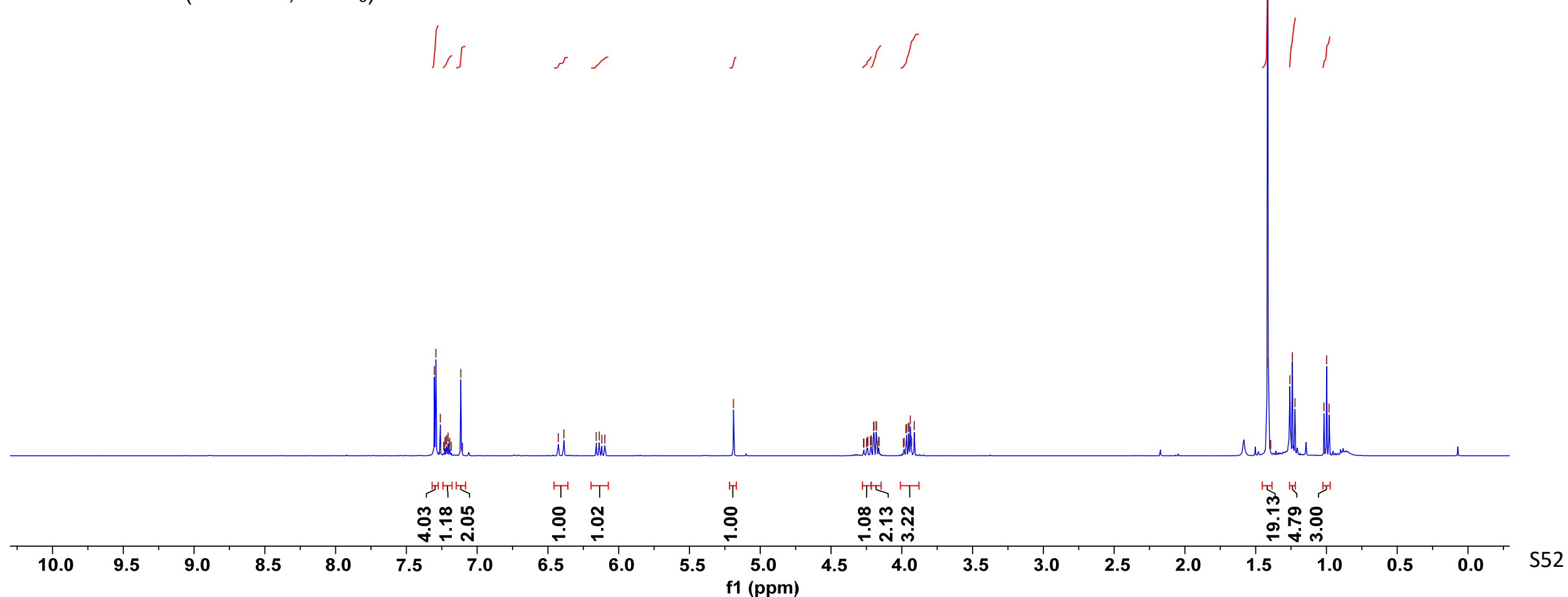




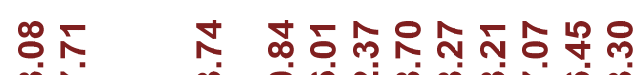

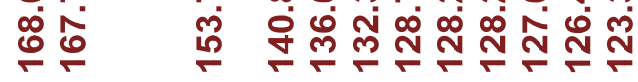

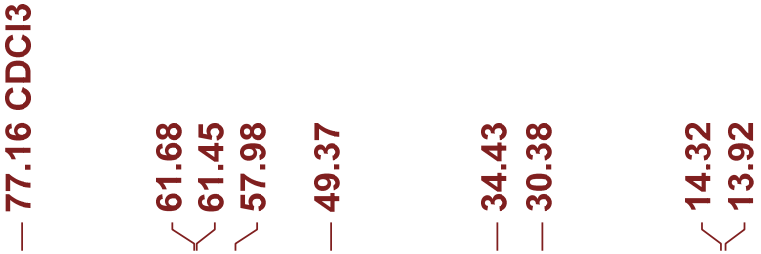

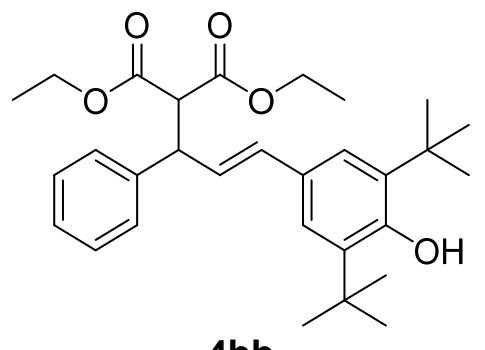

${ }^{13} \mathrm{C}$ NMR $\left(101 \mathrm{MHz}, \mathrm{CDCl}_{3}\right)$

ㅇํㅇ

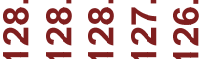
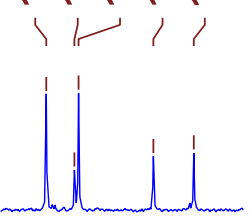

$129128127 \quad 12$

f1 (ppm)
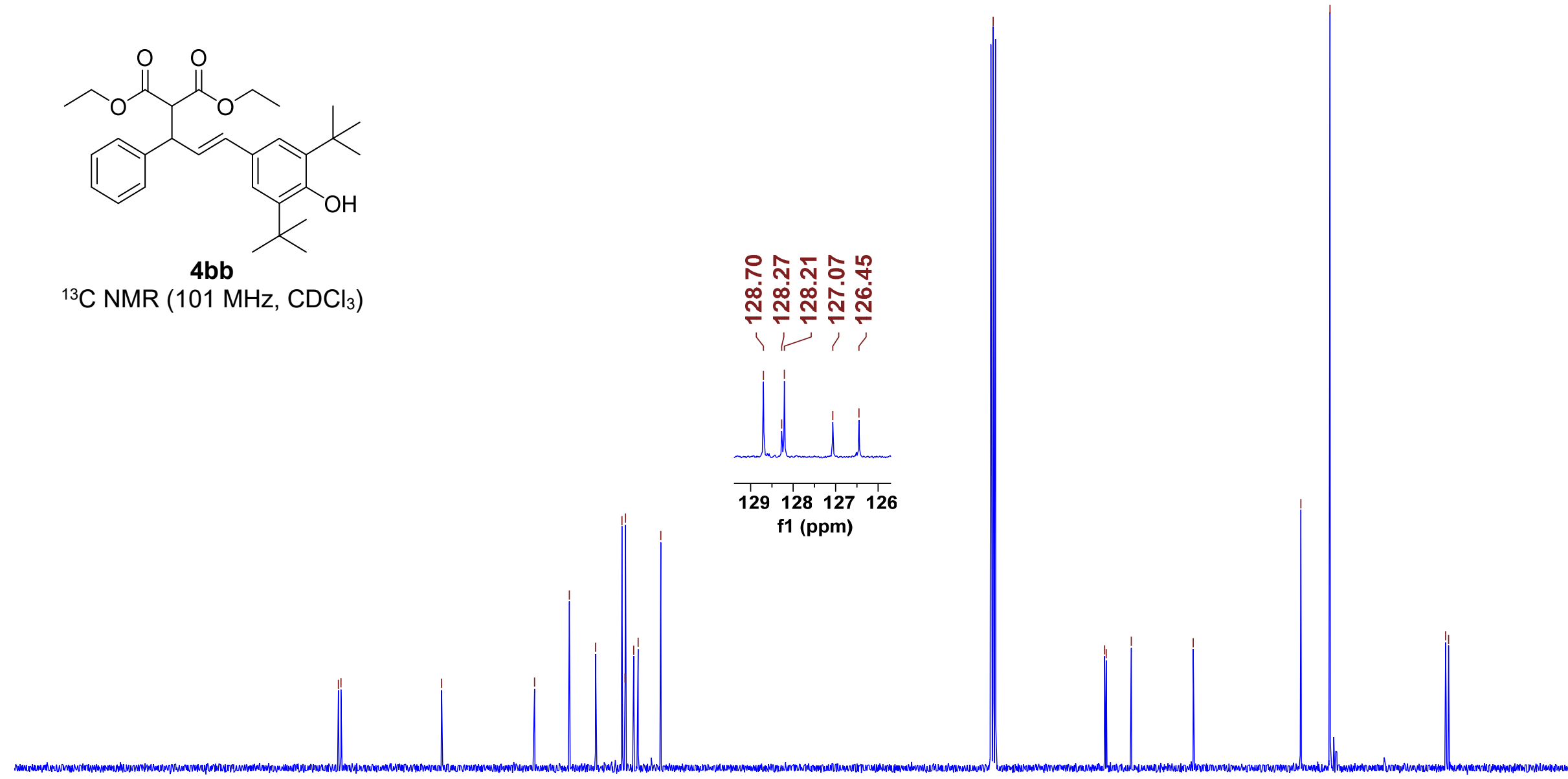
品

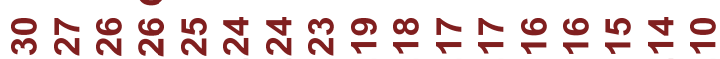

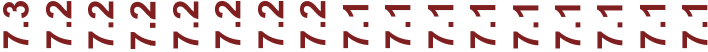

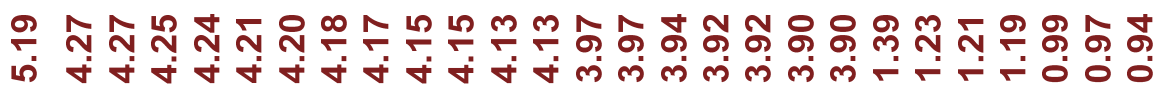

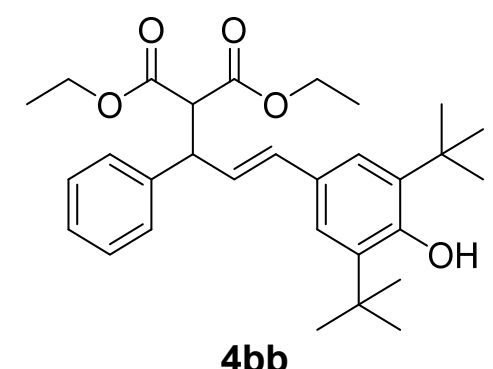

4bb

${ }^{1} \mathrm{H} \mathrm{NMR}\left(300 \mathrm{MHz}, \mathrm{CDCl}_{3}\right)$

Experiment on $1 \mathrm{mmol}$ scale
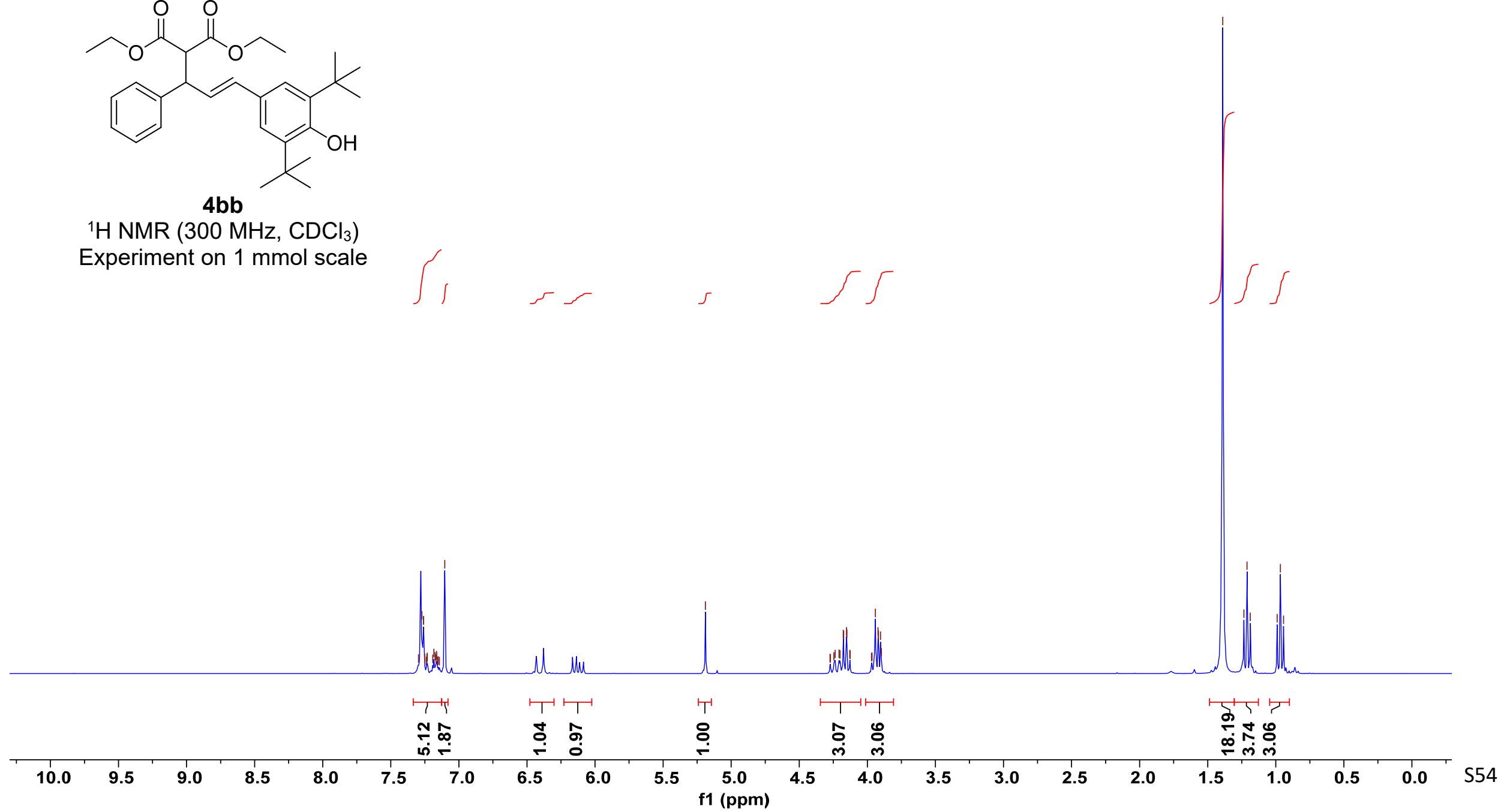


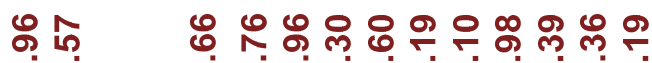

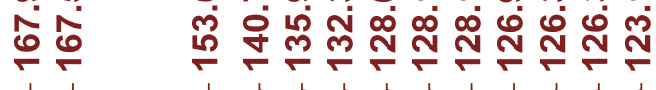
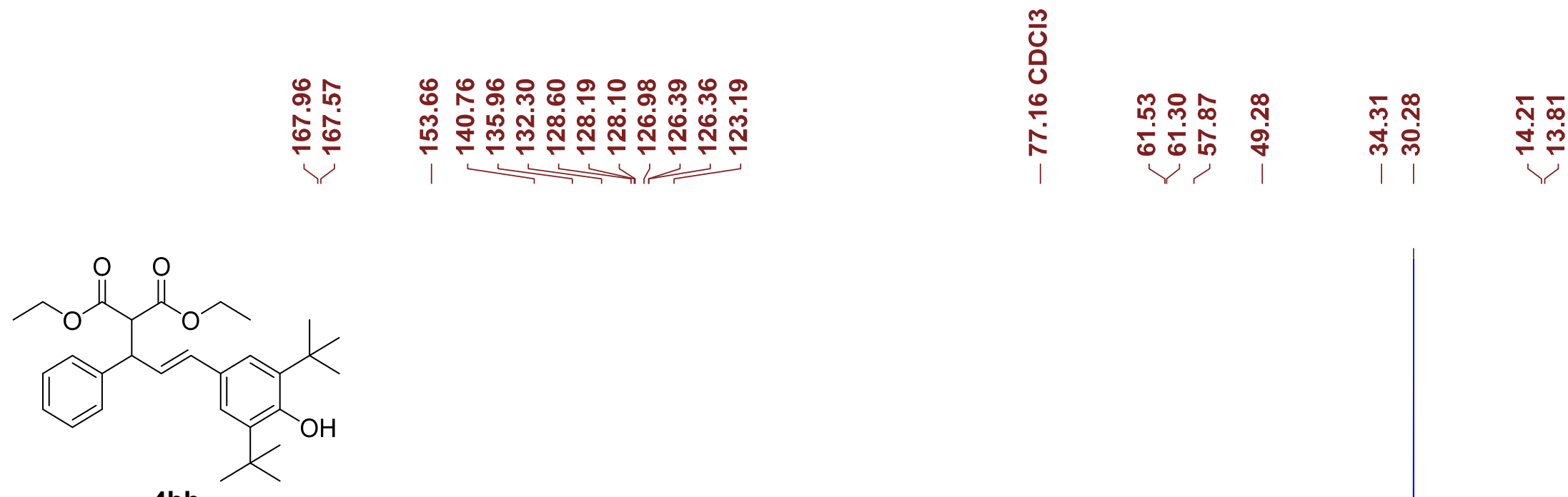

${ }^{13} \mathrm{C} \mathrm{NMR}\left(75.5 \mathrm{MHz}, \mathrm{CDCl}_{3}\right)$

Experiment on $1 \mathrm{mmol}$ scale

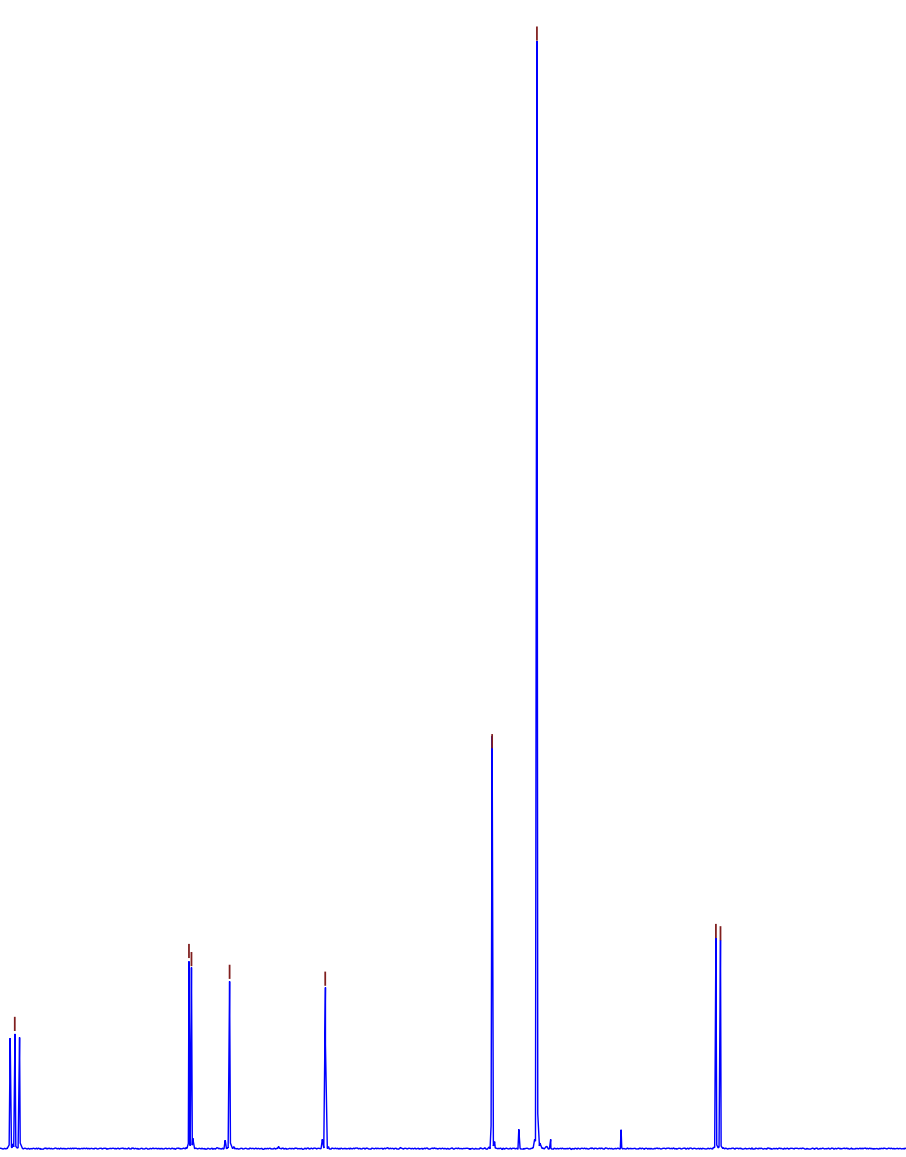




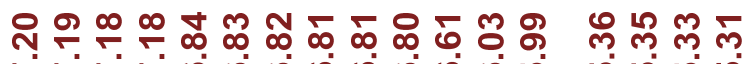

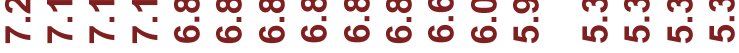
पा

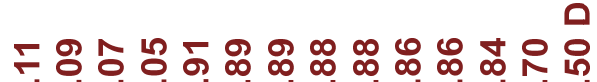
ن

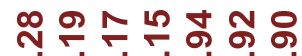

i.ji,

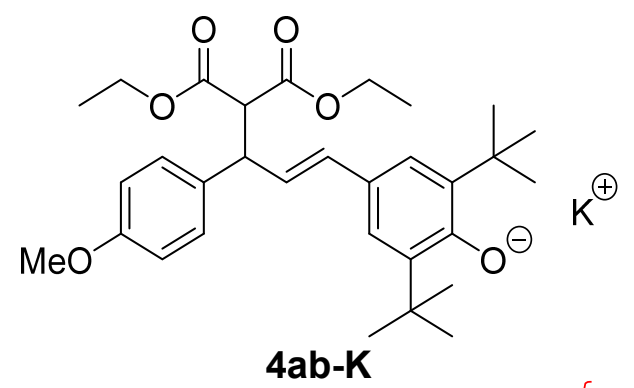

${ }^{1} \mathrm{H}$ NMR (400 MHz, DMSO- $\left.d_{6}\right)$

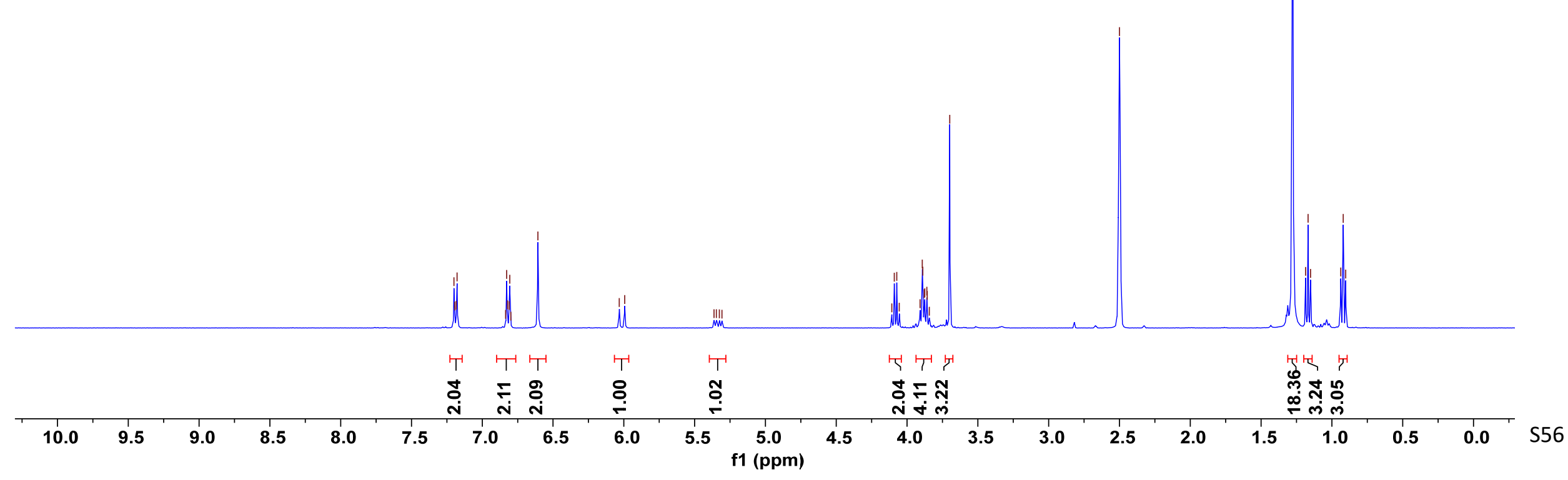




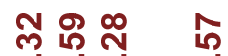

ํํำ ถึ

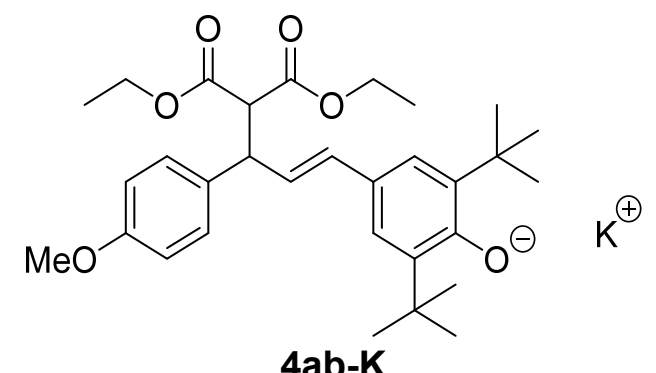

${ }^{13} \mathrm{C}$ NMR (101 MHz, DMSO- $\left.d_{6}\right)$

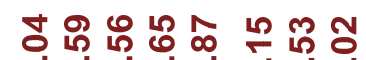

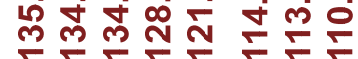

ए।

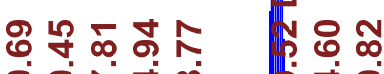

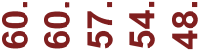

पर

岱

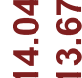

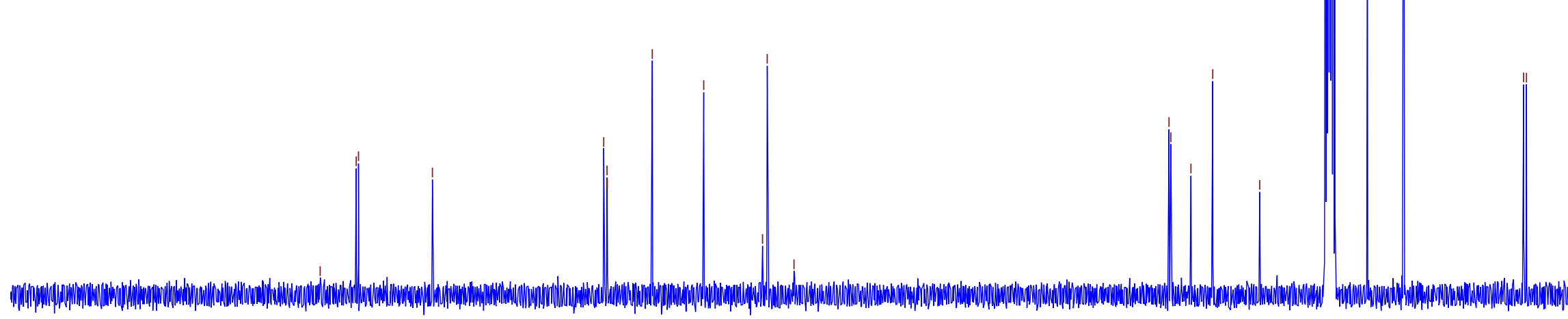




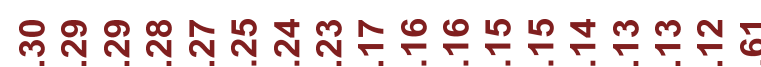

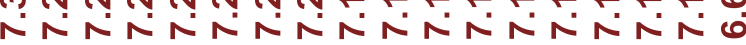

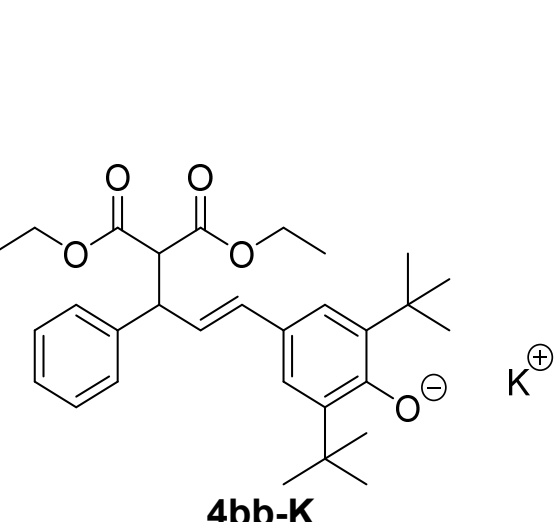

${ }^{1} \mathrm{H}$ NMR $\left(400 \mathrm{MHz}, \mathrm{DMSO}-d_{6}\right)$
동

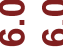

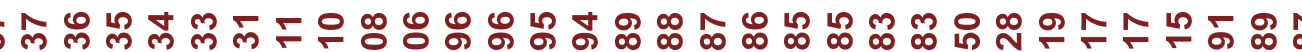

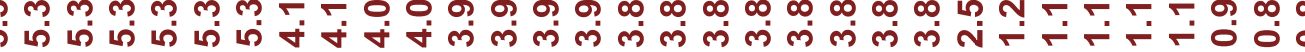

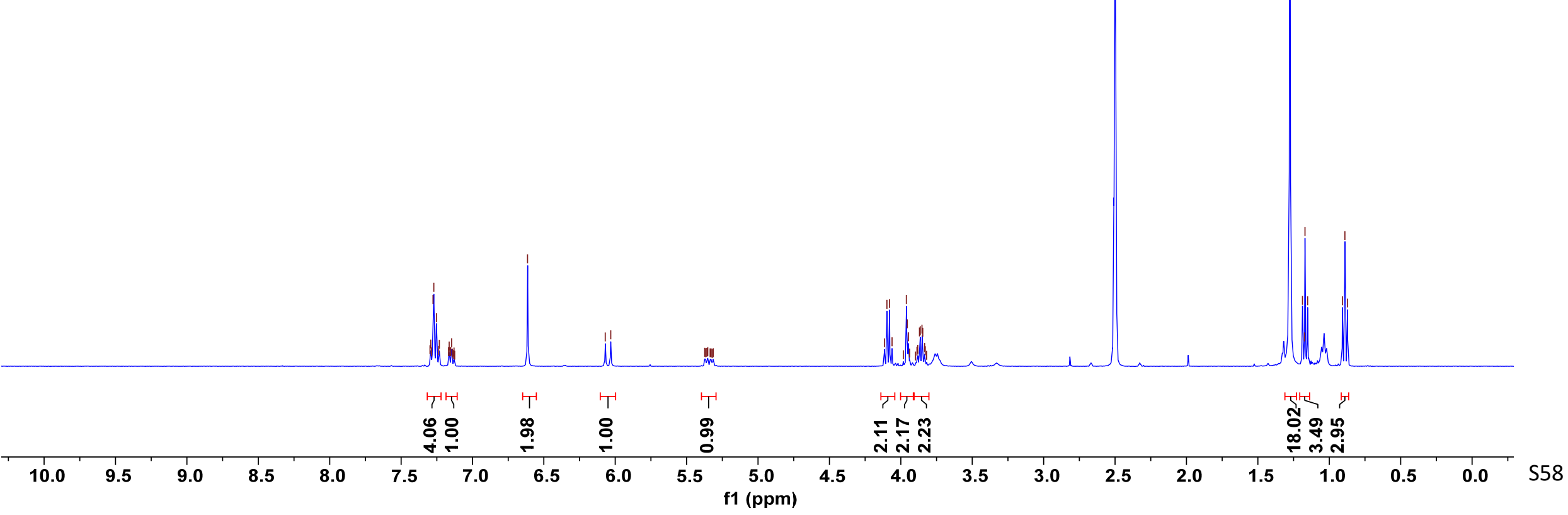


నึ 유 유

승

i

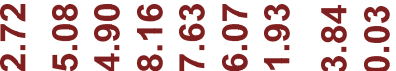

ํำ

।
ำ得早

ठิ 8 in

हैं कि
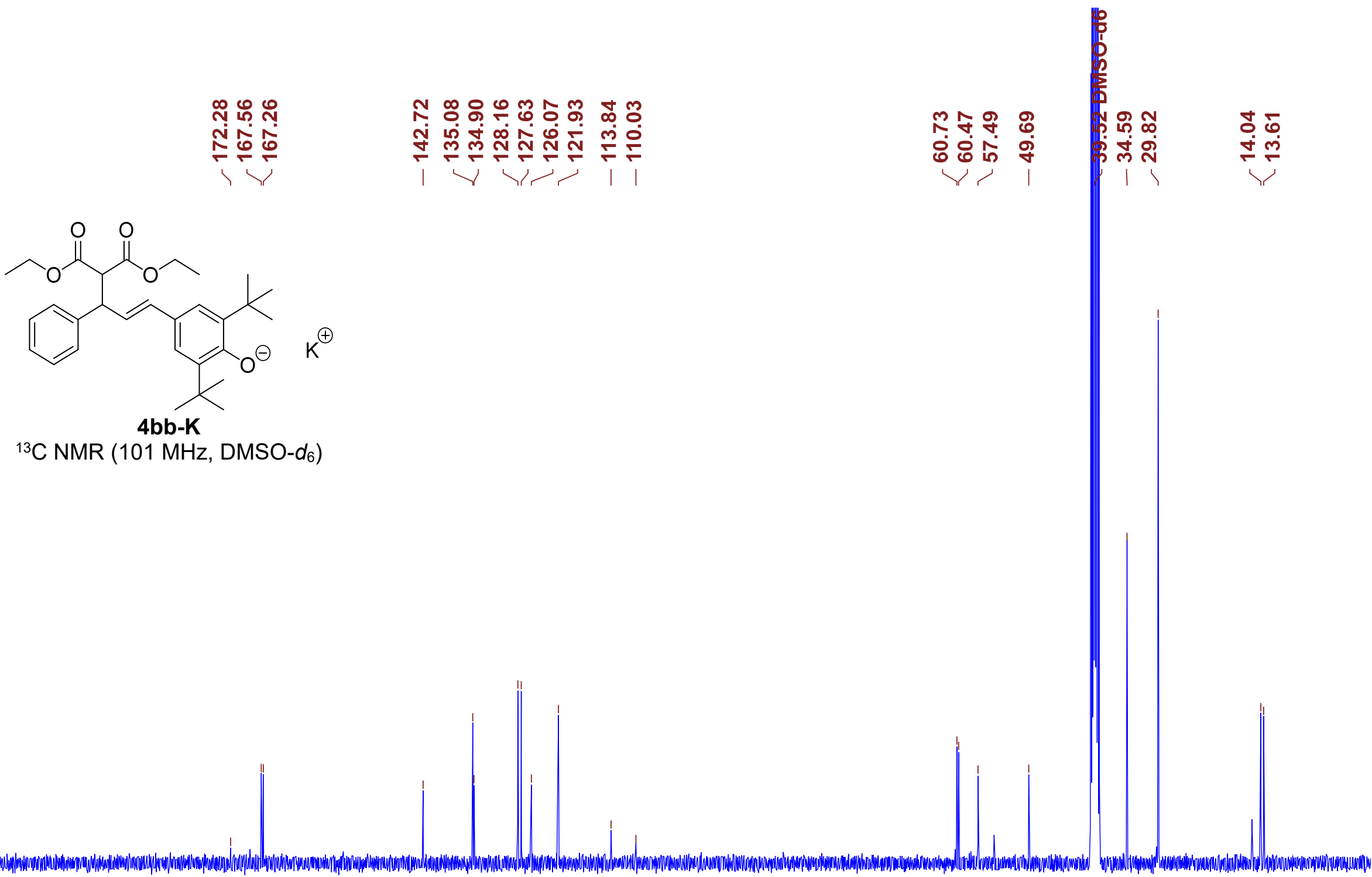

${ }^{13} \mathrm{C}$ NMR (101 MHz, DMSO- $d_{6}$ )

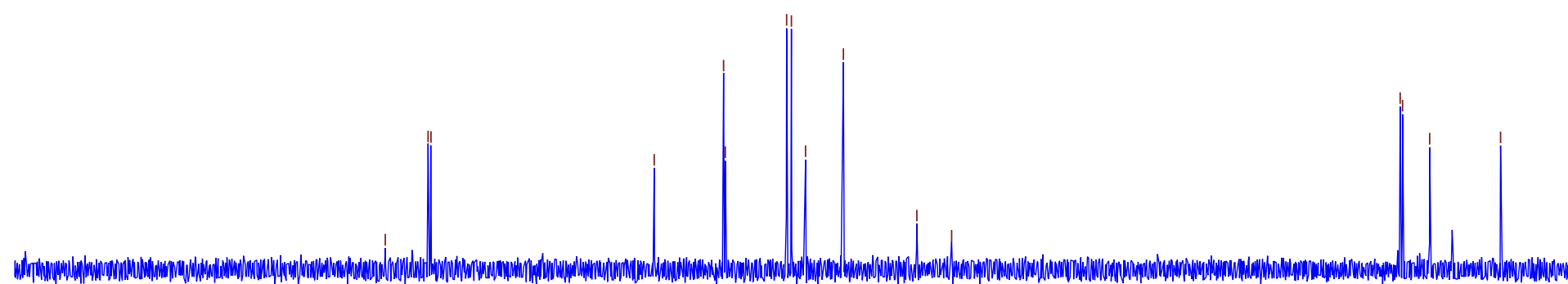




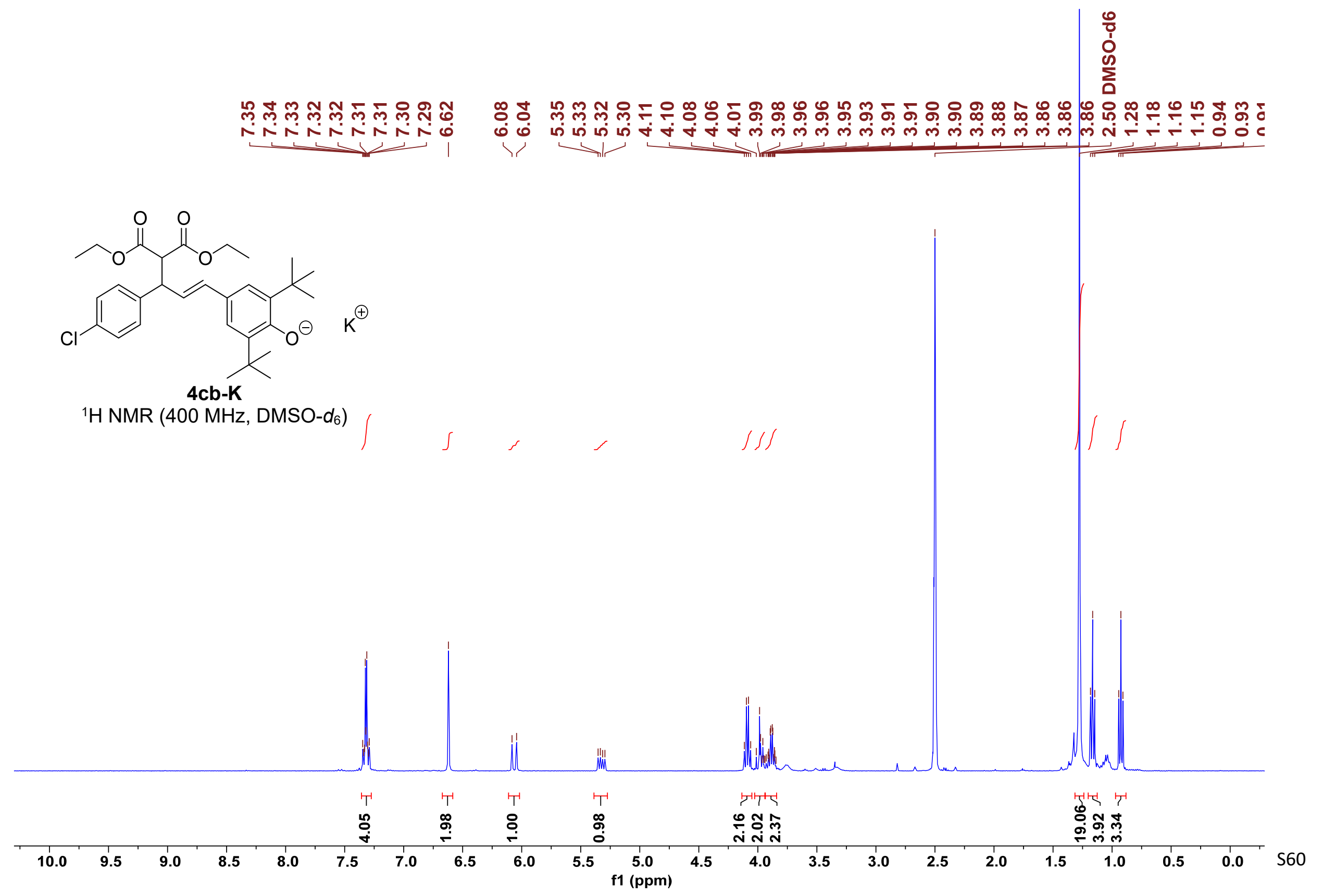




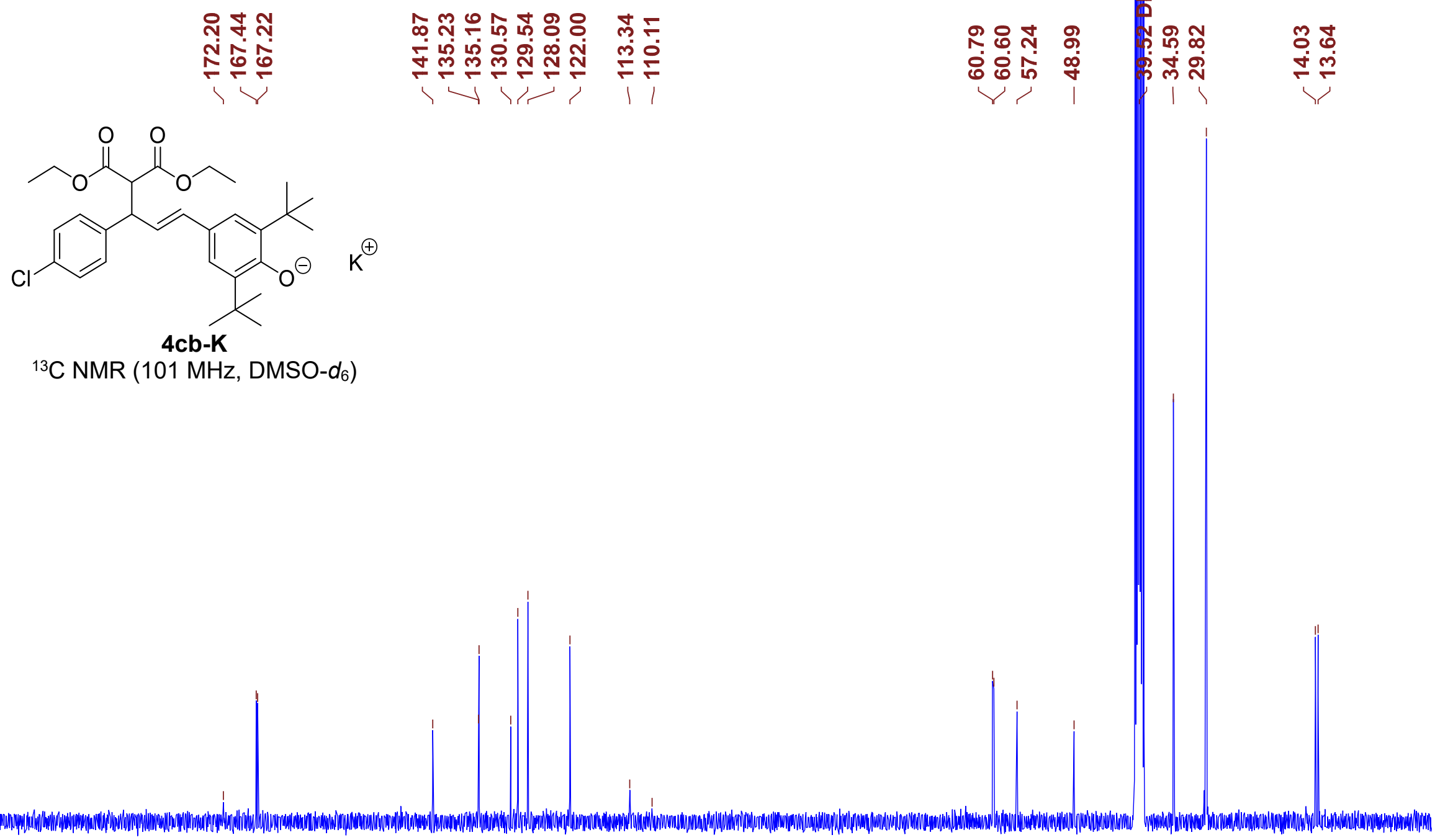

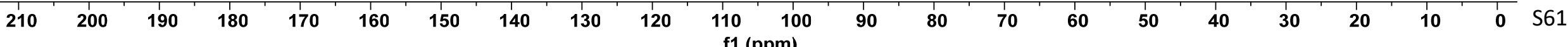




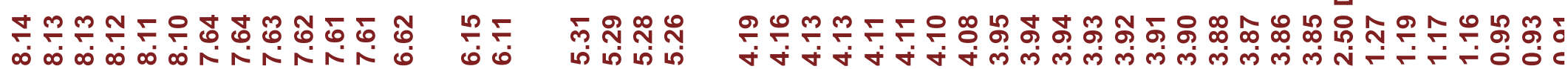

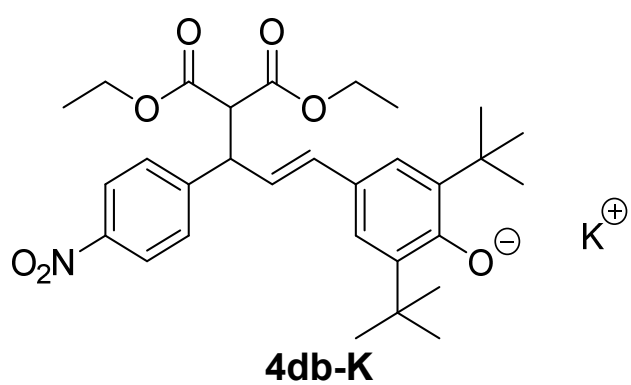

${ }^{1} \mathrm{H}$ NMR (400 MHz, DMSO- $\left.d_{6}\right)$

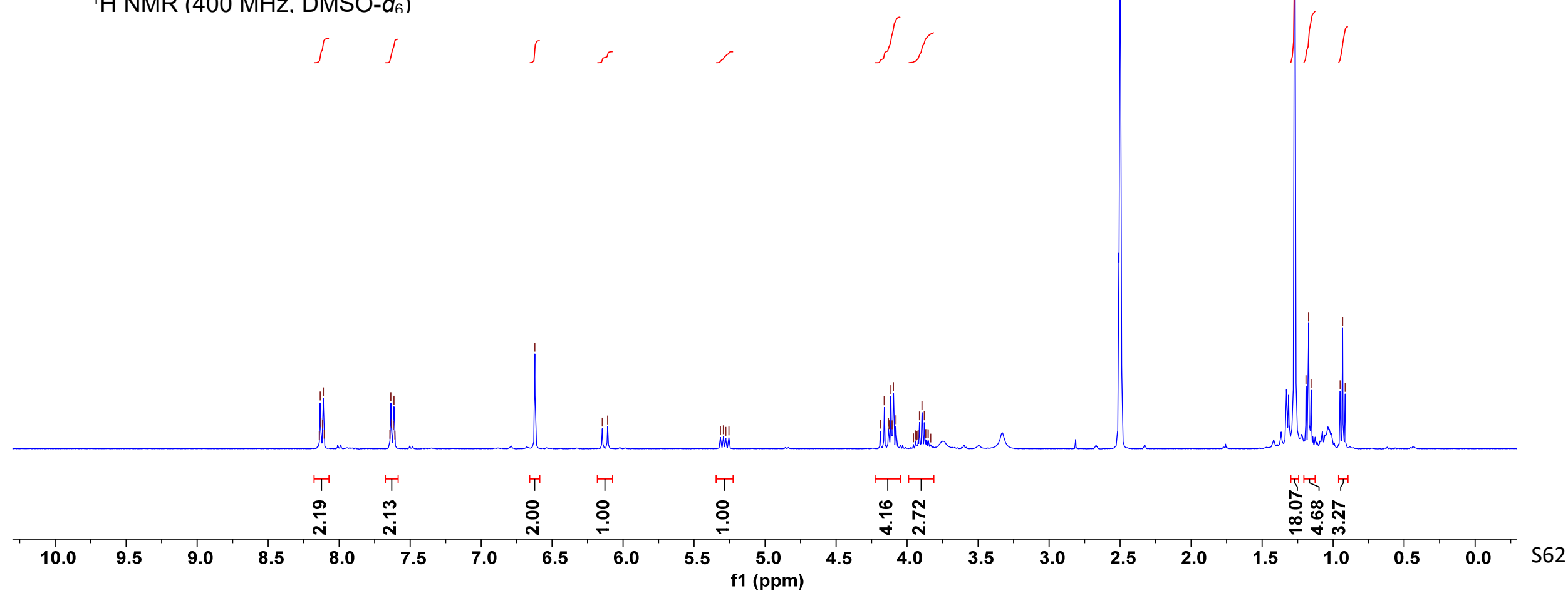




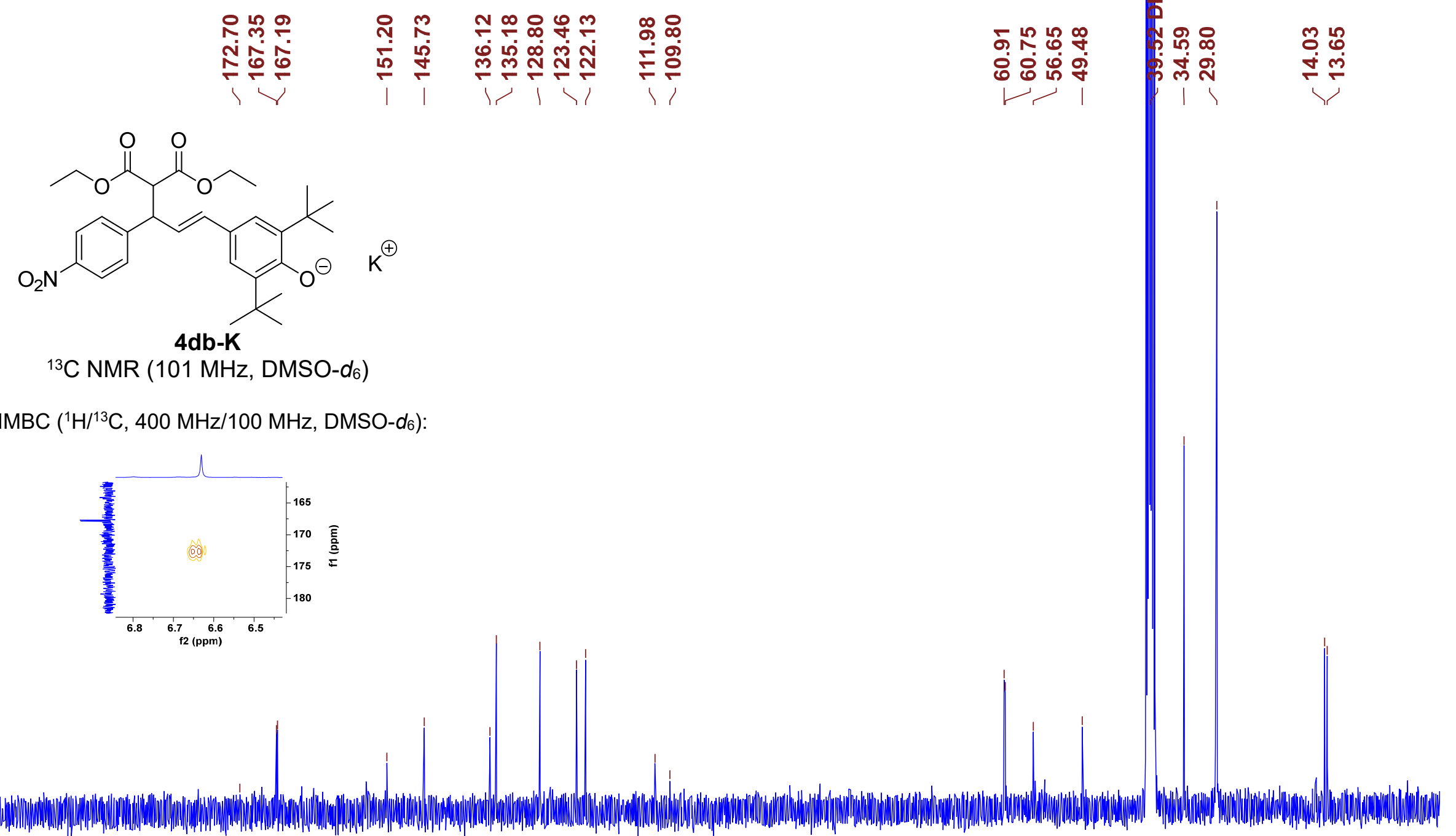

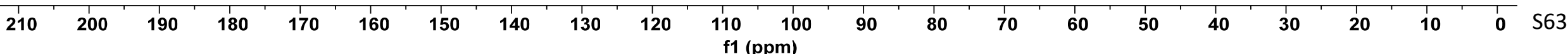


鱼

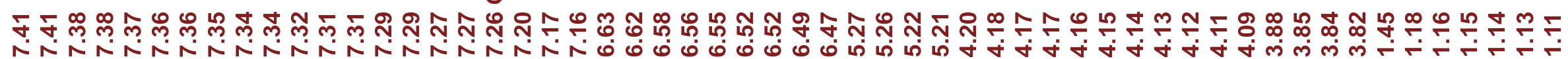

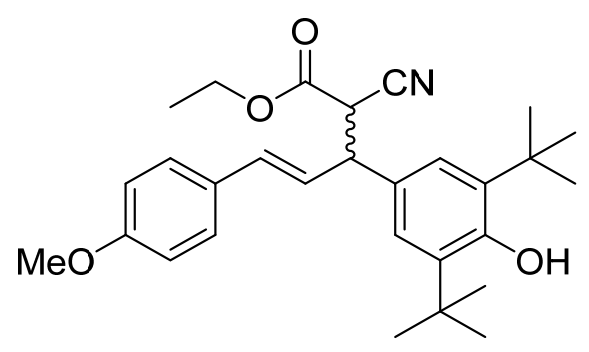

$3 b c$

mixture of diastereoisomers

${ }^{1} \mathrm{H}$ NMR $\left(300 \mathrm{MHz}, \mathrm{CDCl}_{3}\right)$
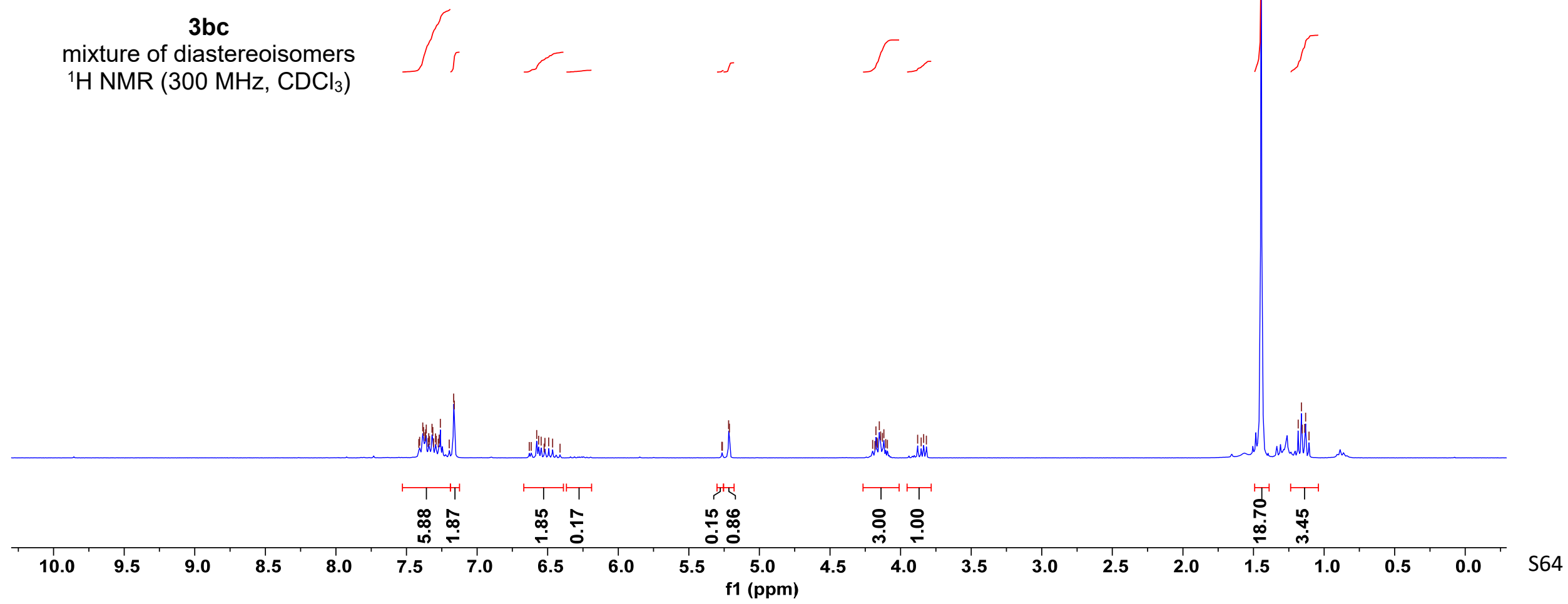


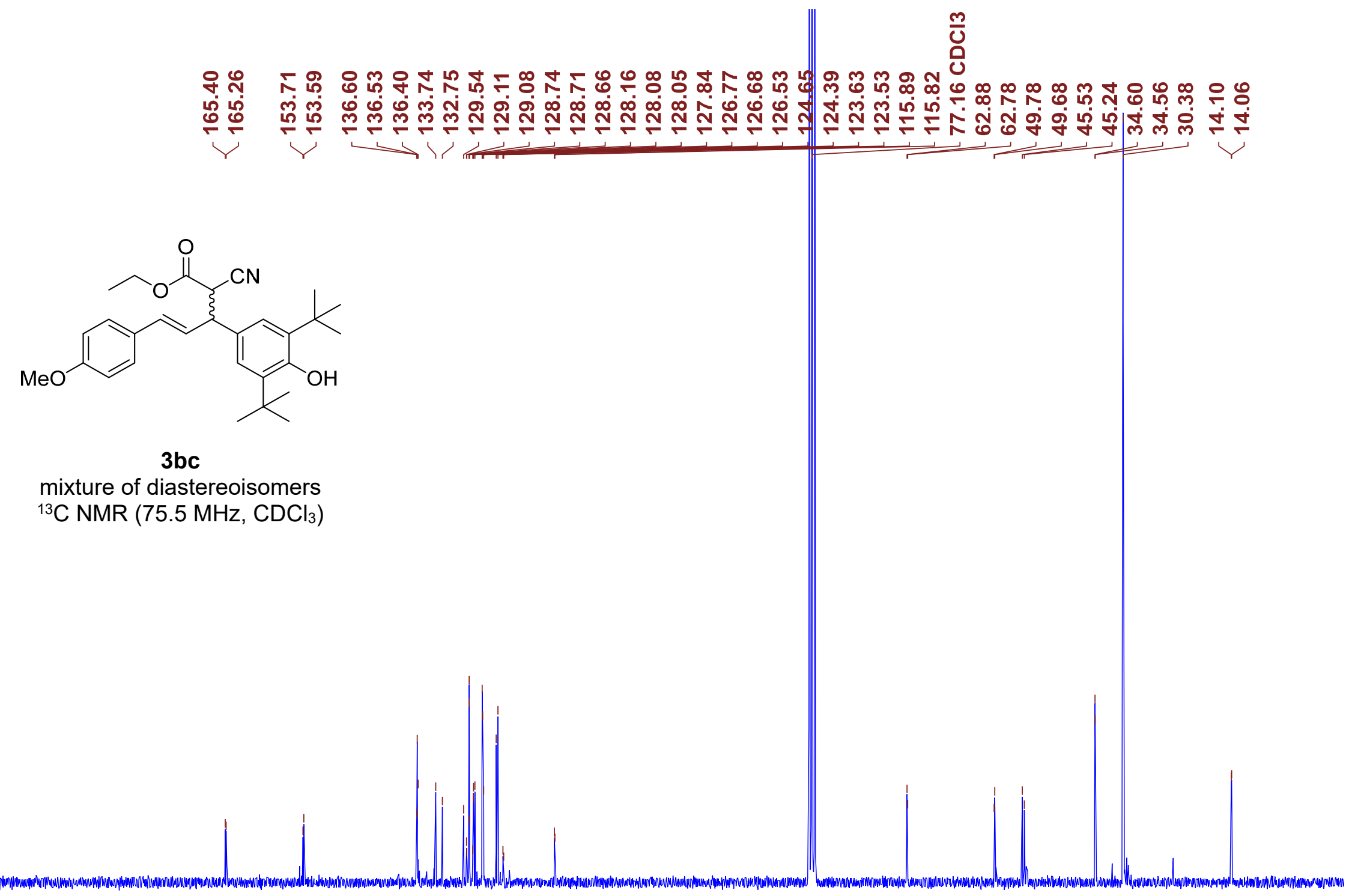


$\frac{m}{0}$

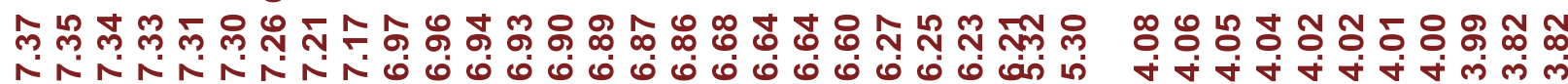

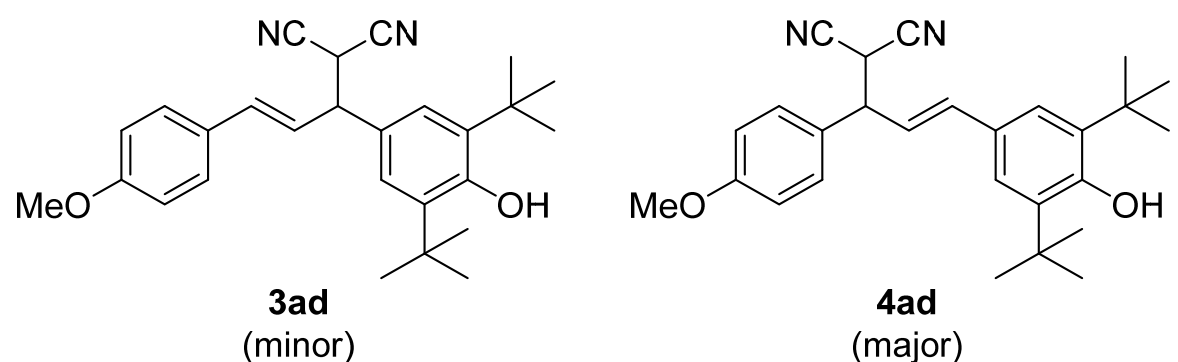

(minor)

mixture of regioisomers

${ }^{1} \mathrm{H}$ NMR $\left(400 \mathrm{MHz}, \mathrm{CDCl}_{3}\right)$

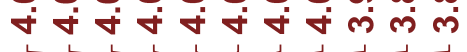
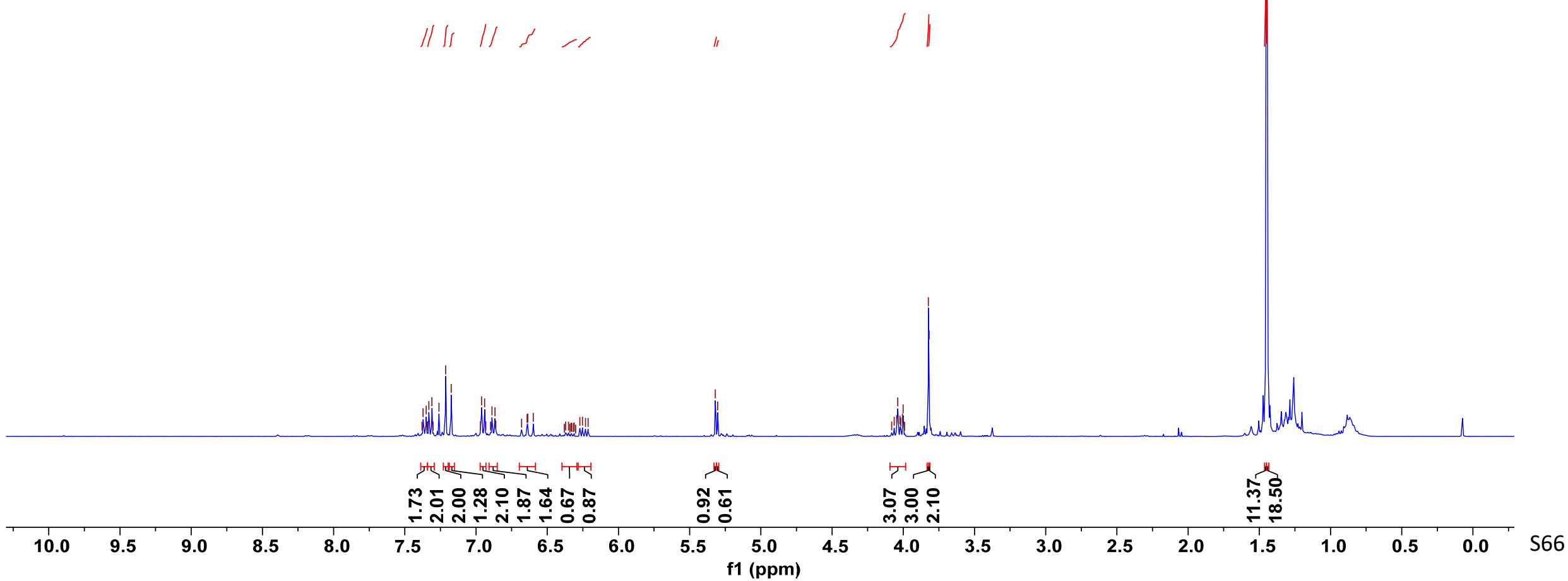


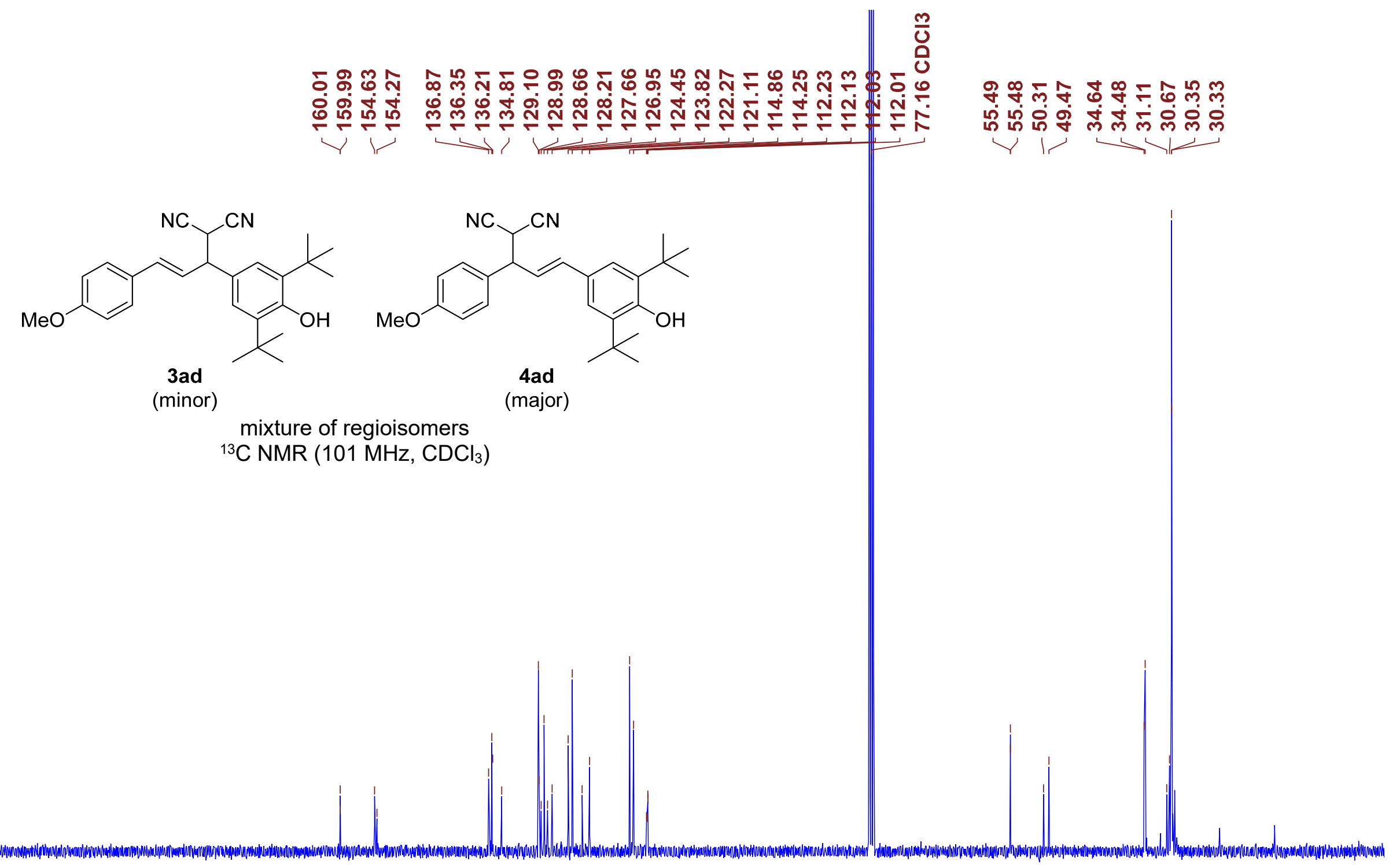

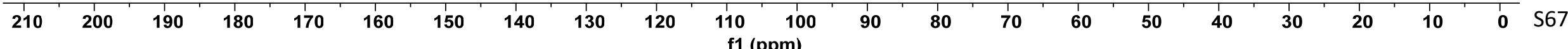




$$
\frac{m}{0}
$$

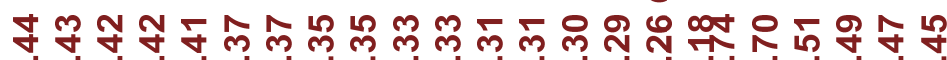

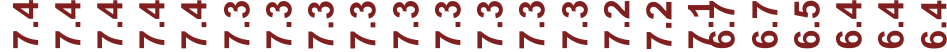

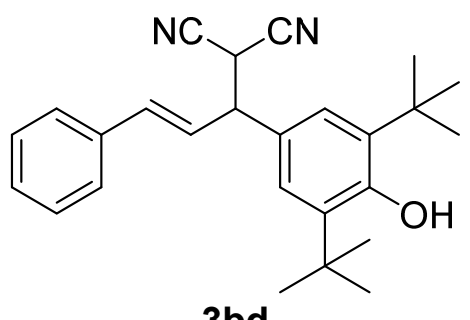

${ }^{1} \mathrm{H}$ NMR $\left(400 \mathrm{MHz}, \mathrm{CDCl}_{3}\right)$

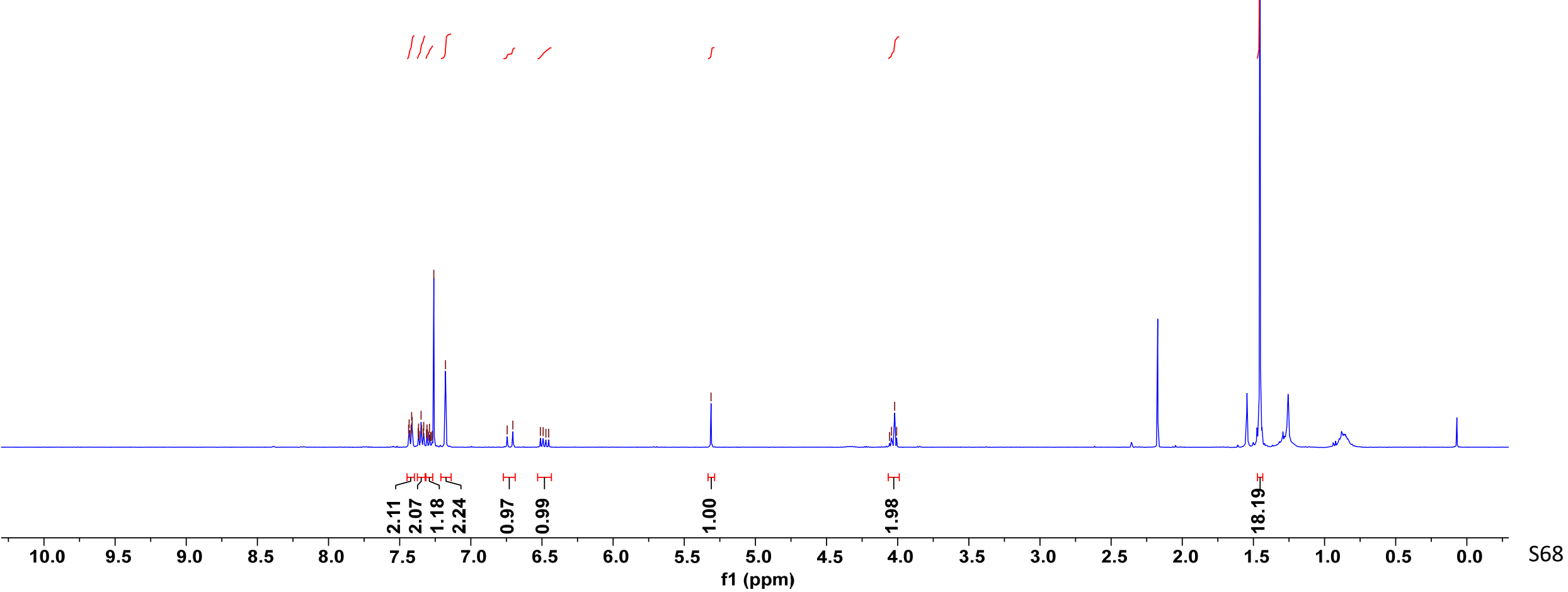


m б ด

ㄴํำ

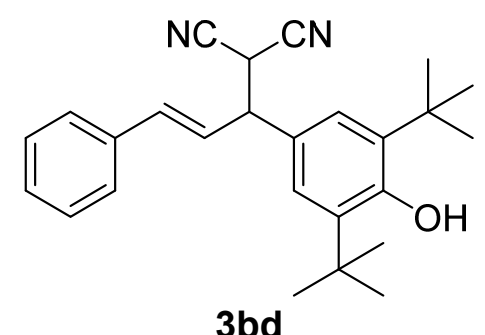

${ }^{13} \mathrm{C}$ NMR $\left(101 \mathrm{MHz}, \mathrm{CDCl}_{3}\right)$
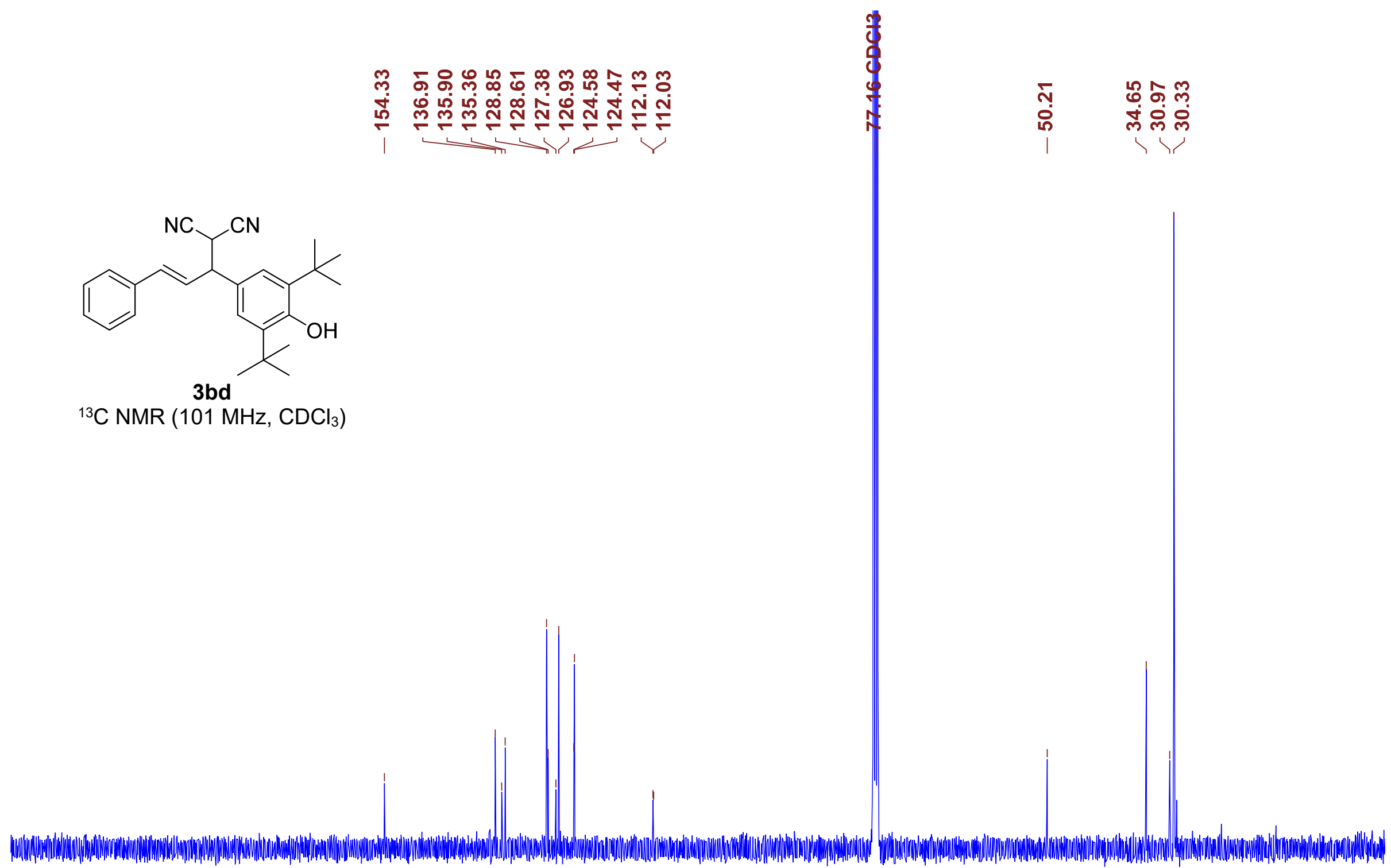

$210 \quad 200$

190

180

170

160

150

140

130

120 $110 \quad 100$

$90 \quad 80$

70

60

50

40

$30 \quad 20$

10 - 569 


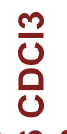

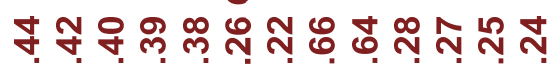

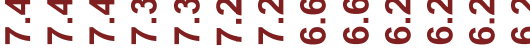

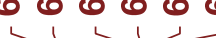

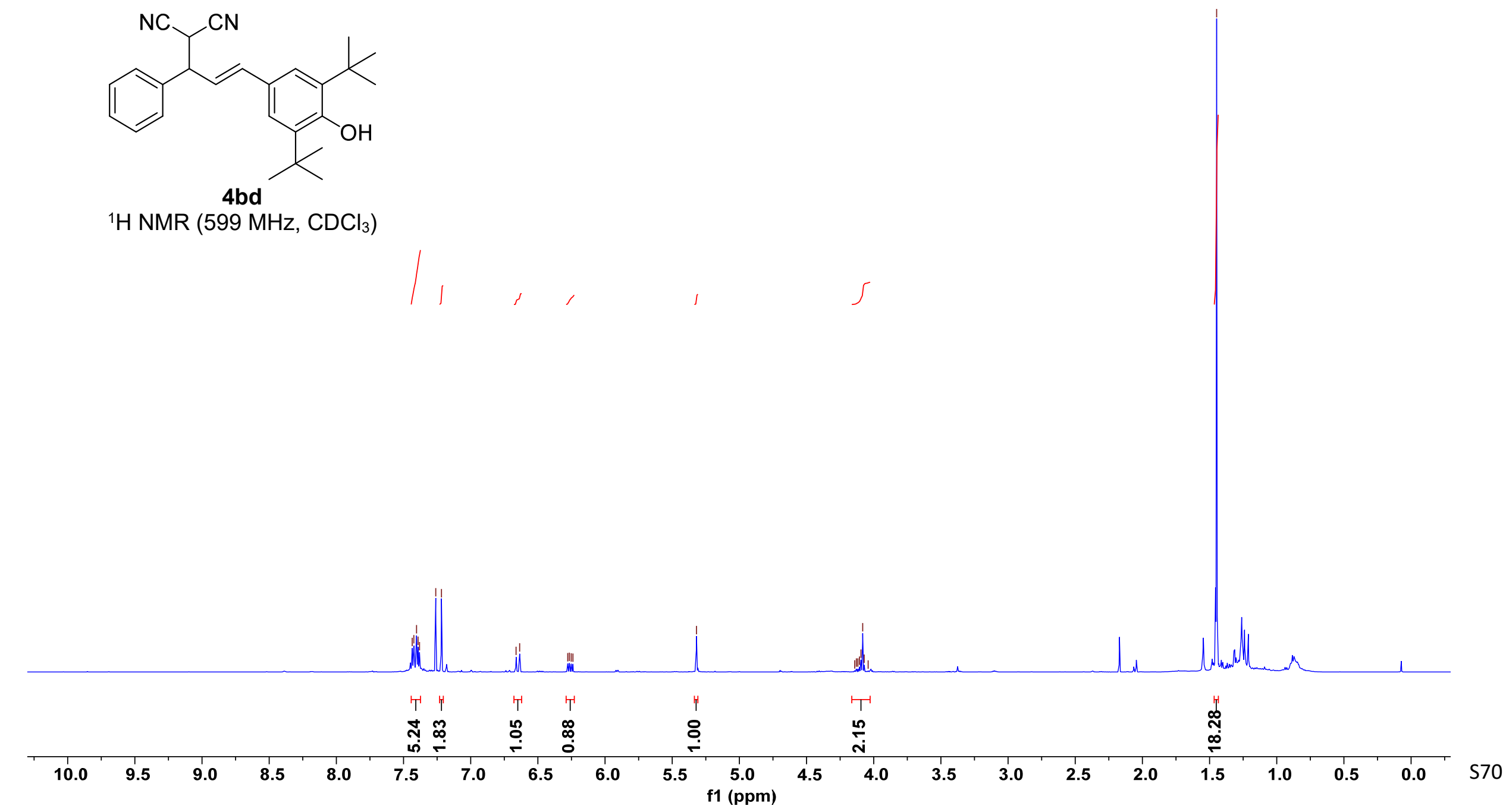

${ }^{1} \mathrm{H} \mathrm{NMR}\left(599 \mathrm{MHz}, \mathrm{CDCl}_{3}\right)$ 


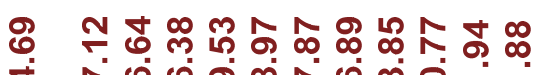

苦

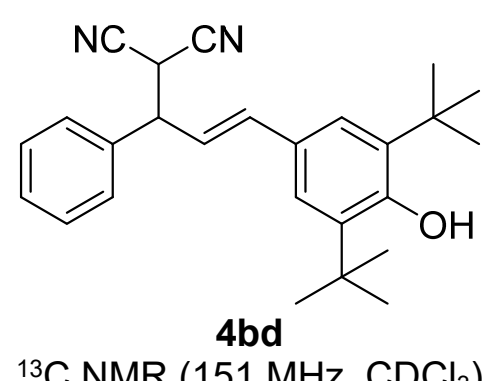

${ }^{13} \mathrm{C}$ NMR (151 MHz, $\mathrm{CDCl}_{3}$ ) 
鱼

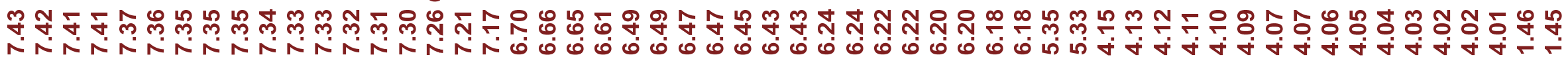
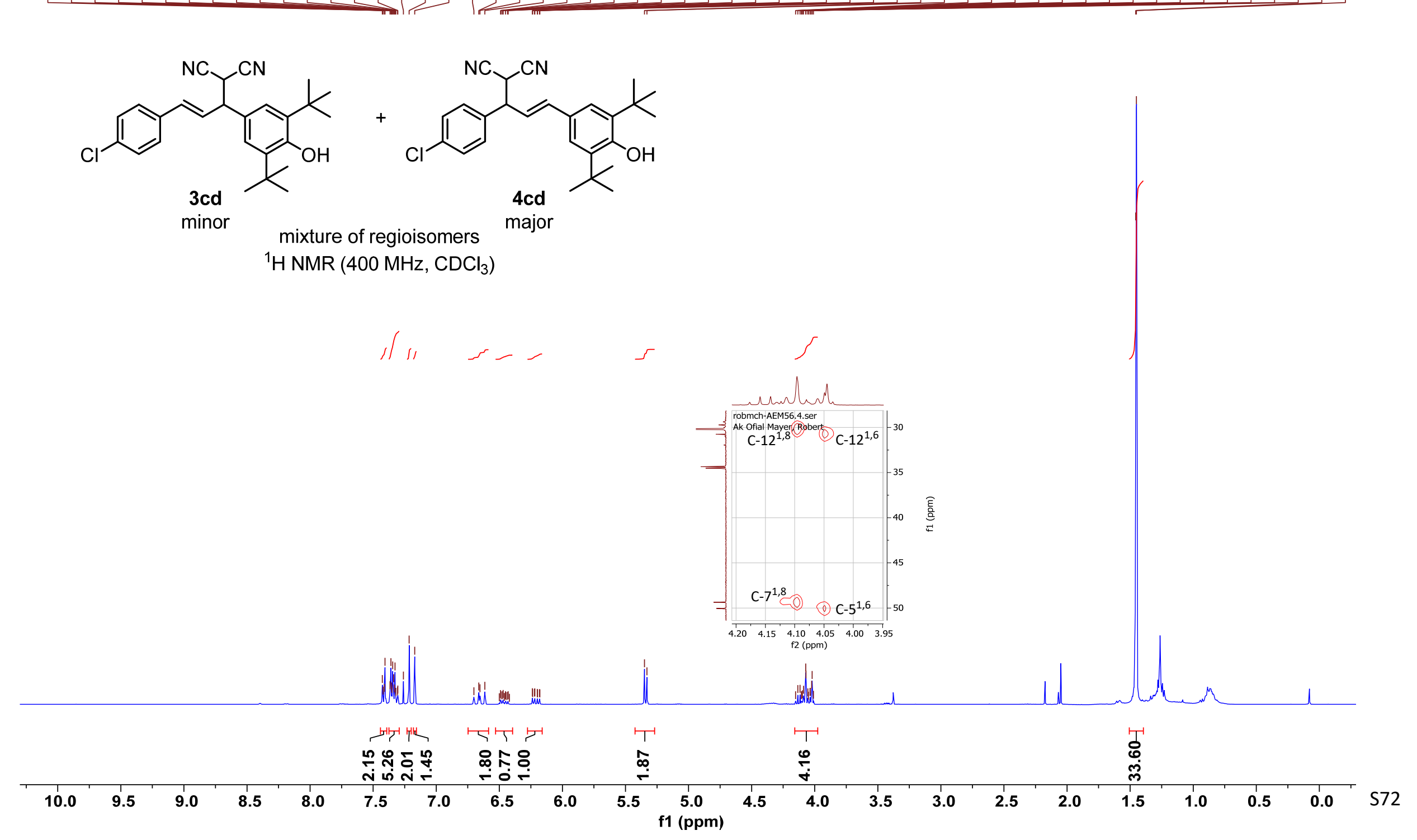


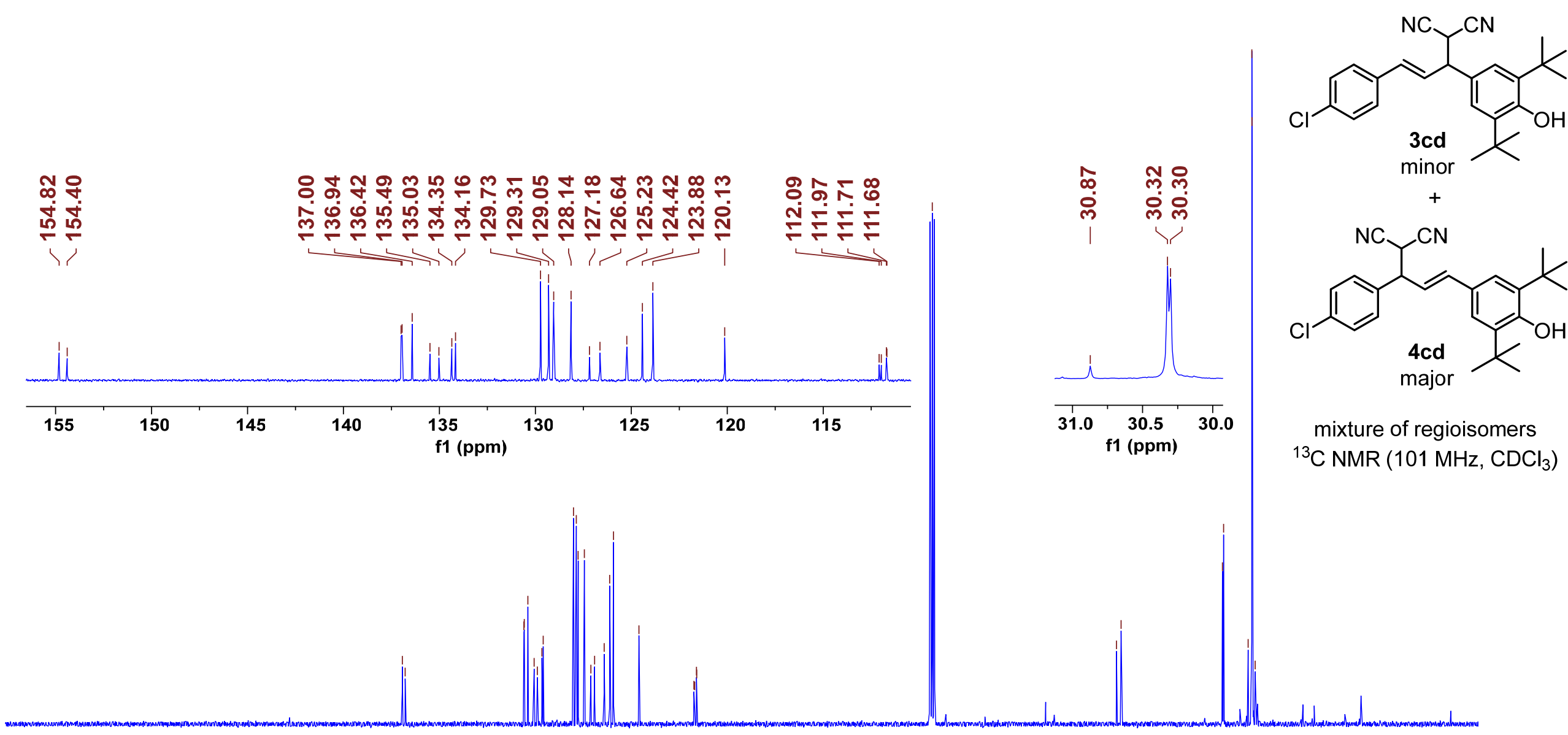


응

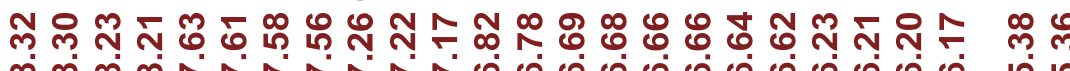

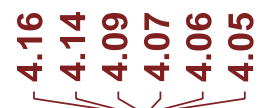

尔

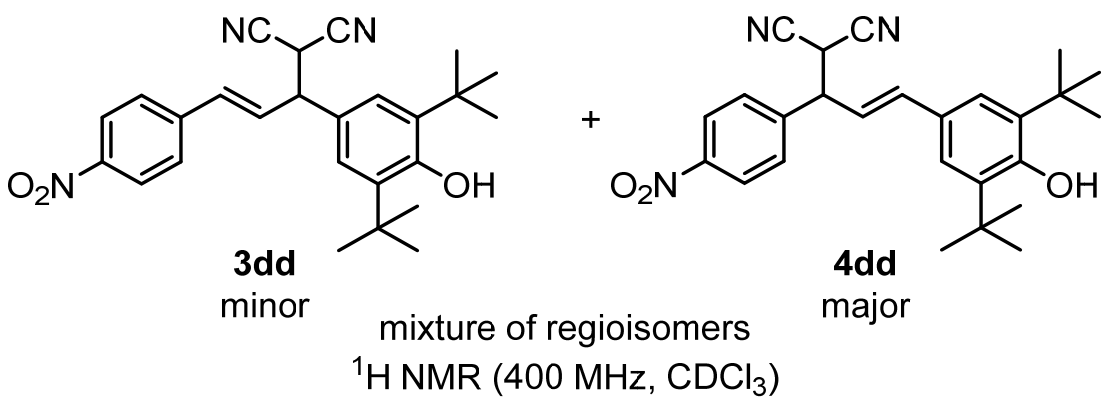

${ }^{1} \mathrm{H} \mathrm{NMR}\left(400 \mathrm{MHz}, \mathrm{CDCl}_{3}\right)$

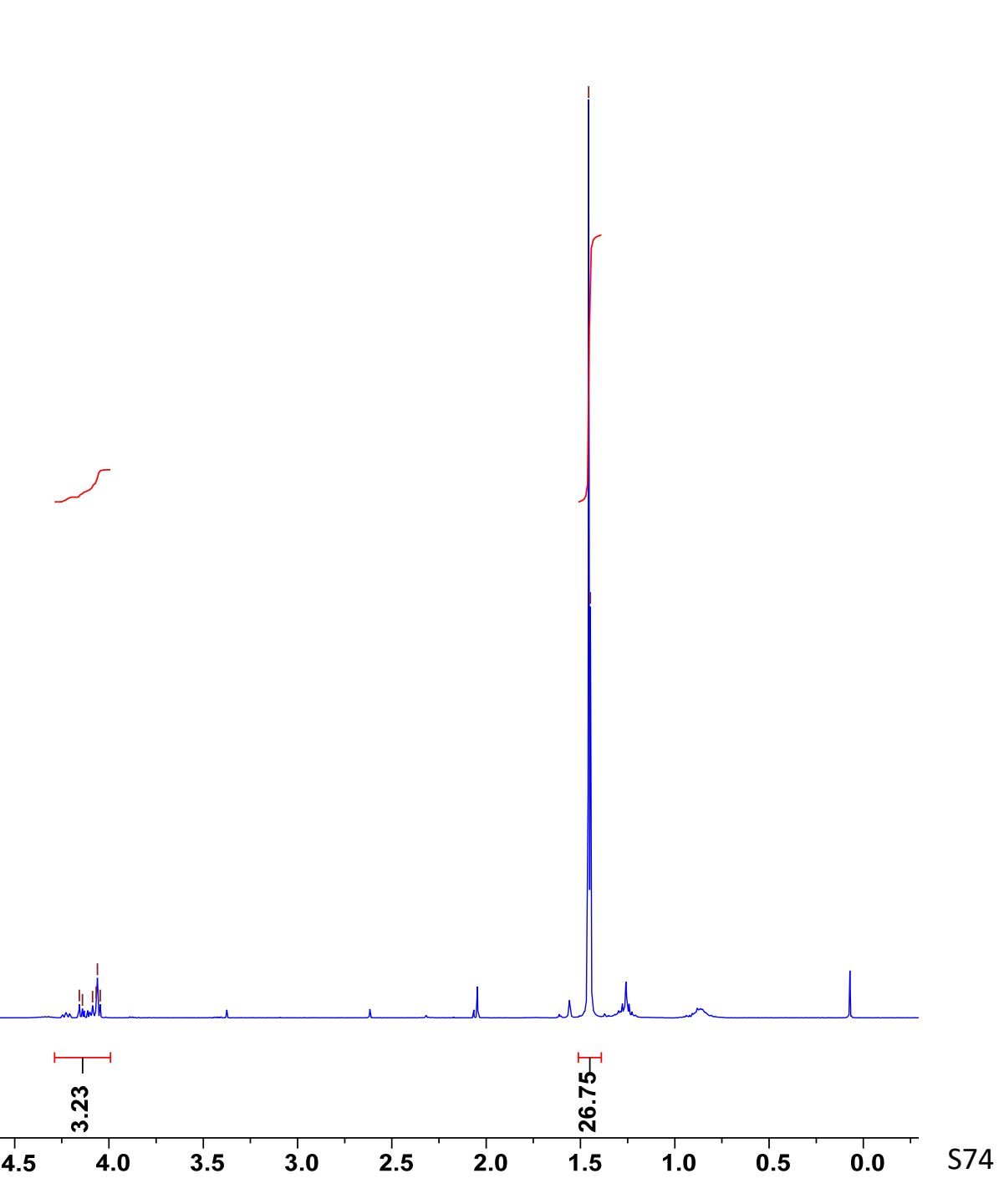




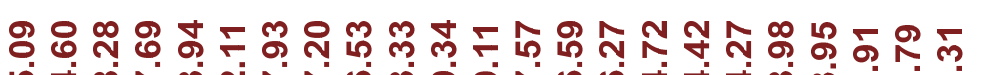

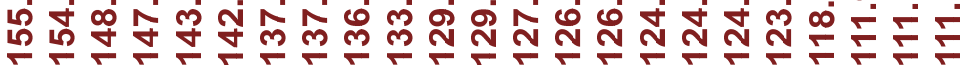

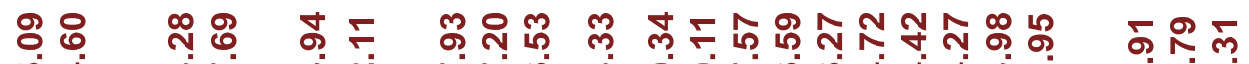

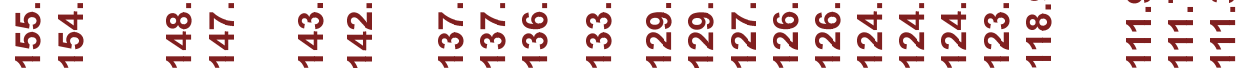

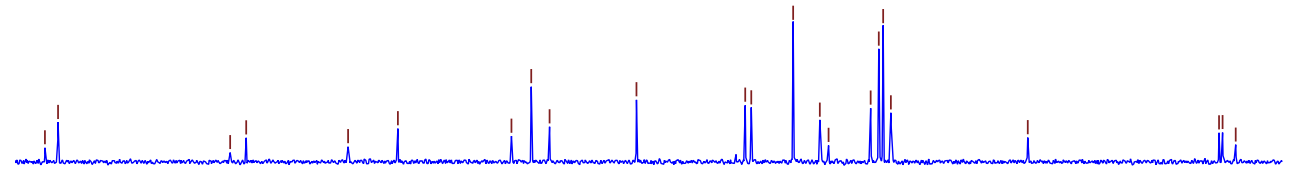$$
155
$$$$
150
$$$$
145
$$
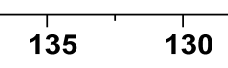

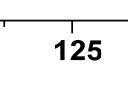

120
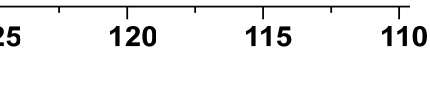

需

เก่
กาก่

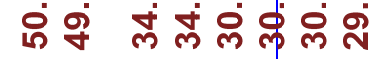

$\checkmark$ 证

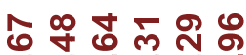

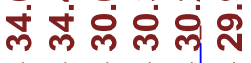
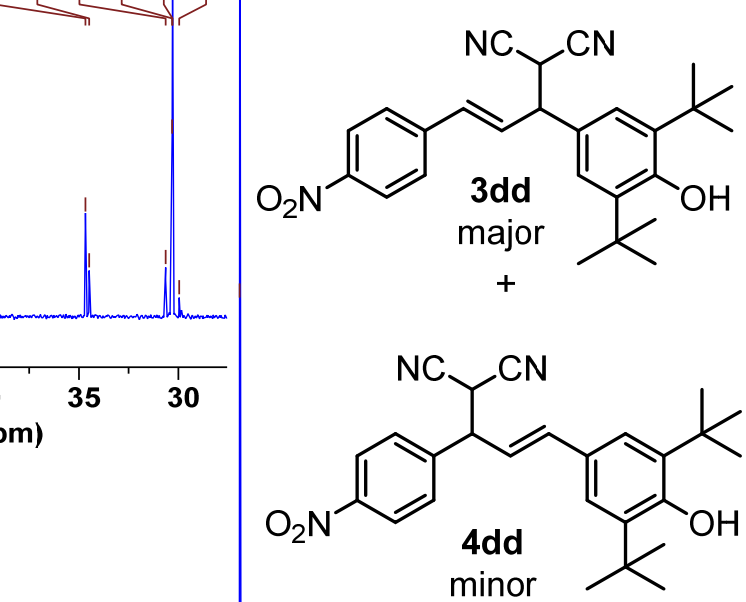

mixture of regioisomers

${ }^{13} \mathrm{C}$ NMR $\left(101 \mathrm{MHz}, \mathrm{CDCl}_{3}\right)$ 

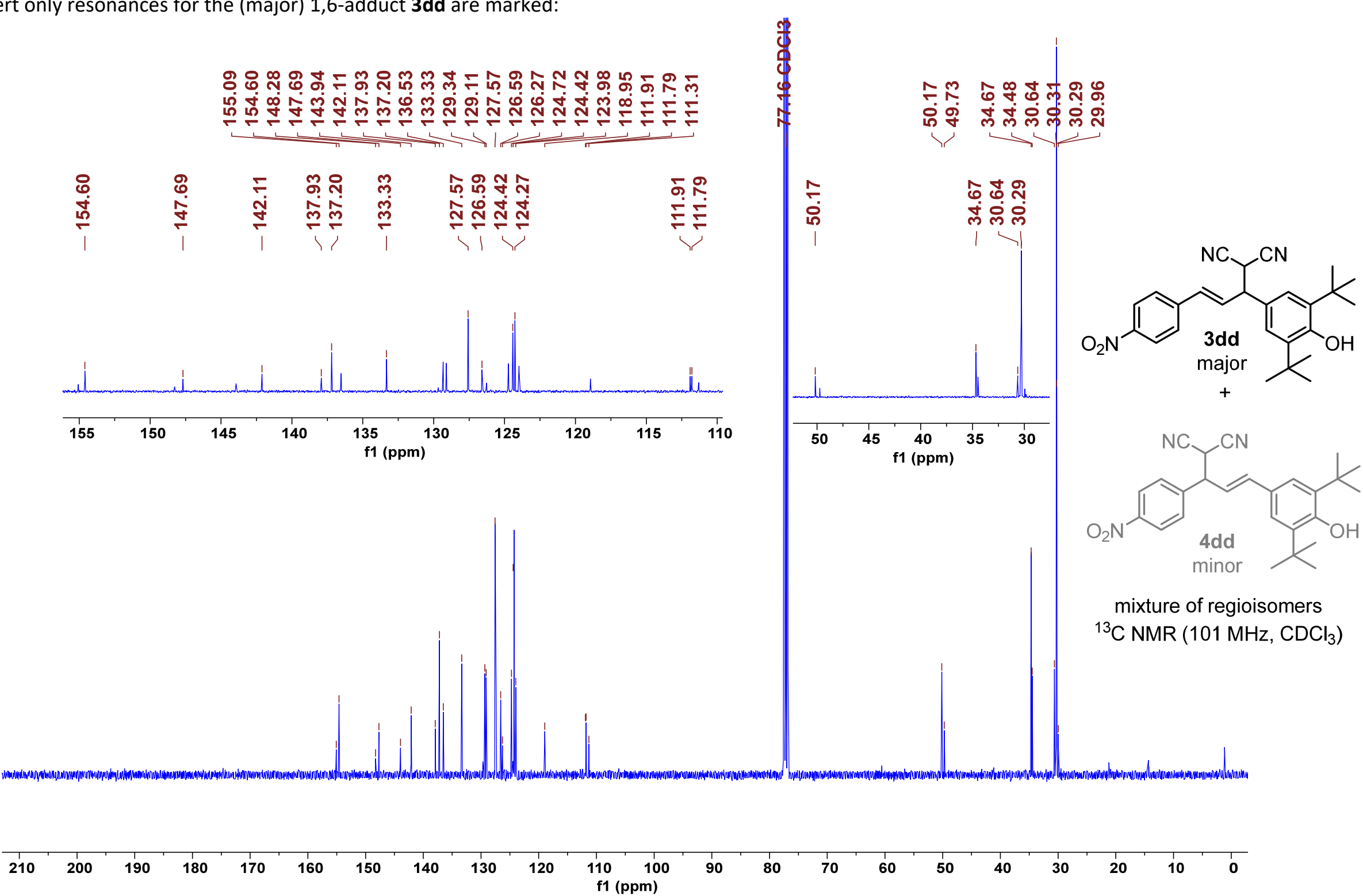
In the insert only resonances for the (minor) 1,8-adduct $\mathbf{4 d d}$ are marked:

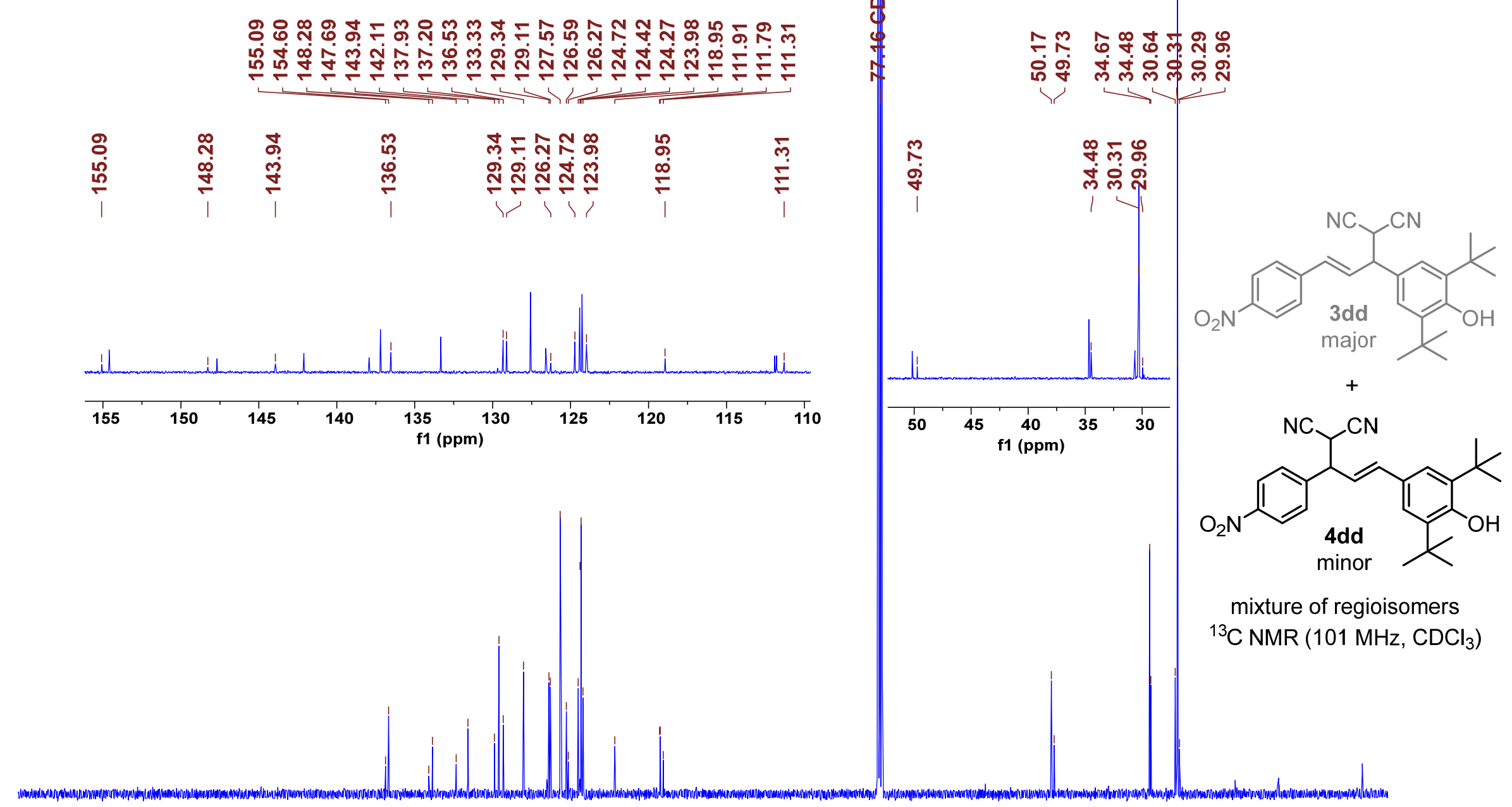

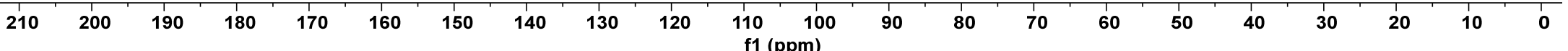


응

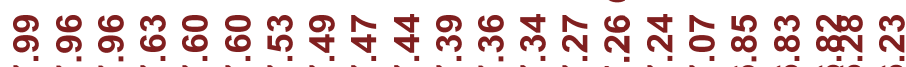

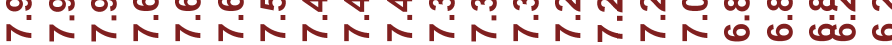

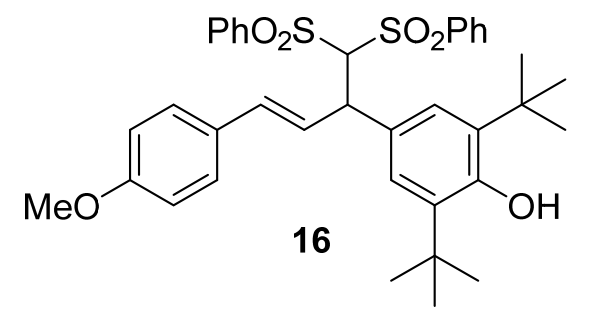

${ }^{1} \mathrm{HNMR}\left(300 \mathrm{MHz}, \mathrm{CDCl}_{3}\right)$
மூ

储, 


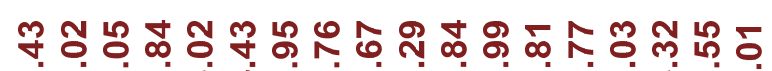
命

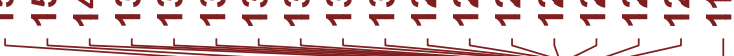

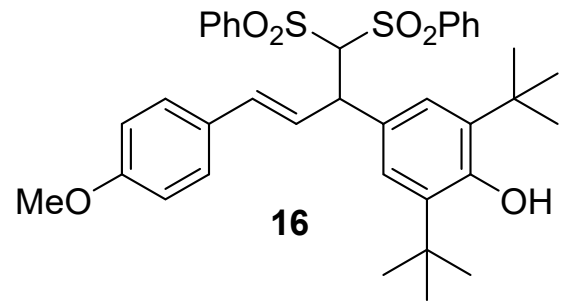

${ }^{13} \mathrm{C} \mathrm{NMR}\left(75.5 \mathrm{MHz}, \mathrm{CDCl}_{3}\right)$

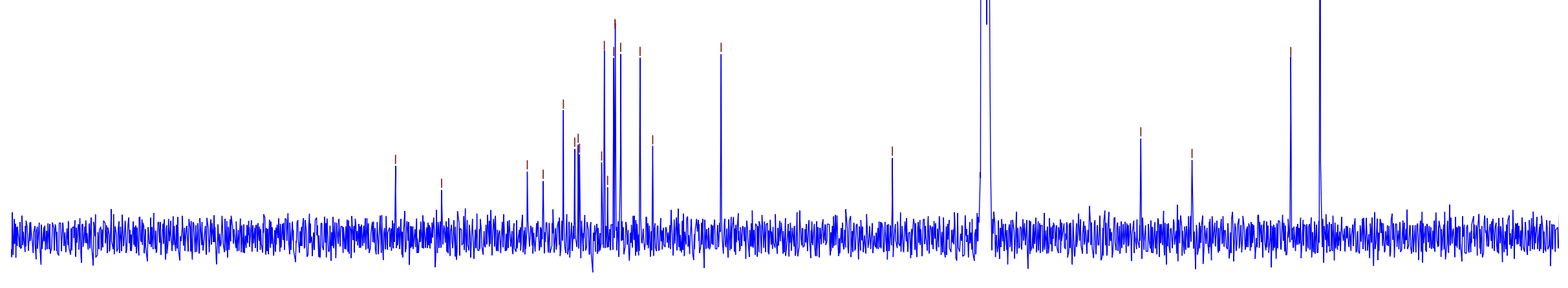

$\begin{array}{rlllllllllllllllllllllllll}210 & 200 & 190 & 180 & 170 & 160 & 150 & 140 & 130 & 120 & 110 & 100 & 90 & 80 & 70 & 60 & 50 & 40 & 30 & 20 & 10 & 0 & S 79\end{array}$


ํํำ

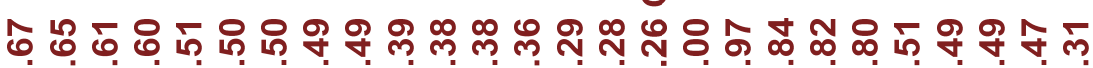

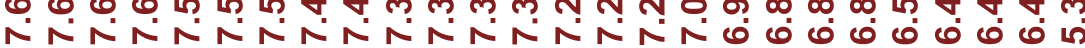

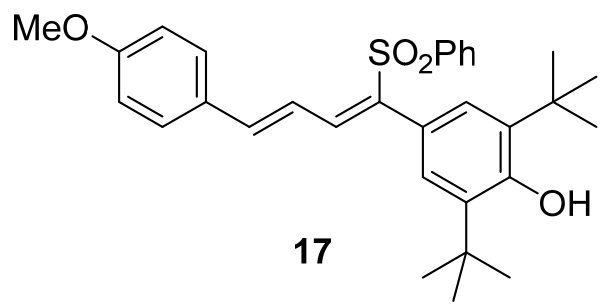

${ }^{1} \mathrm{H}$ NMR $\left(700 \mathrm{MHz}, \mathrm{CDCl}_{3}\right)$

$$
\text { 品 }
$$

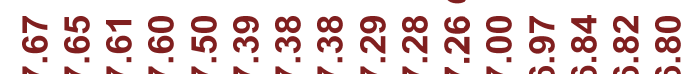

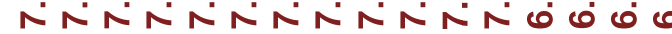
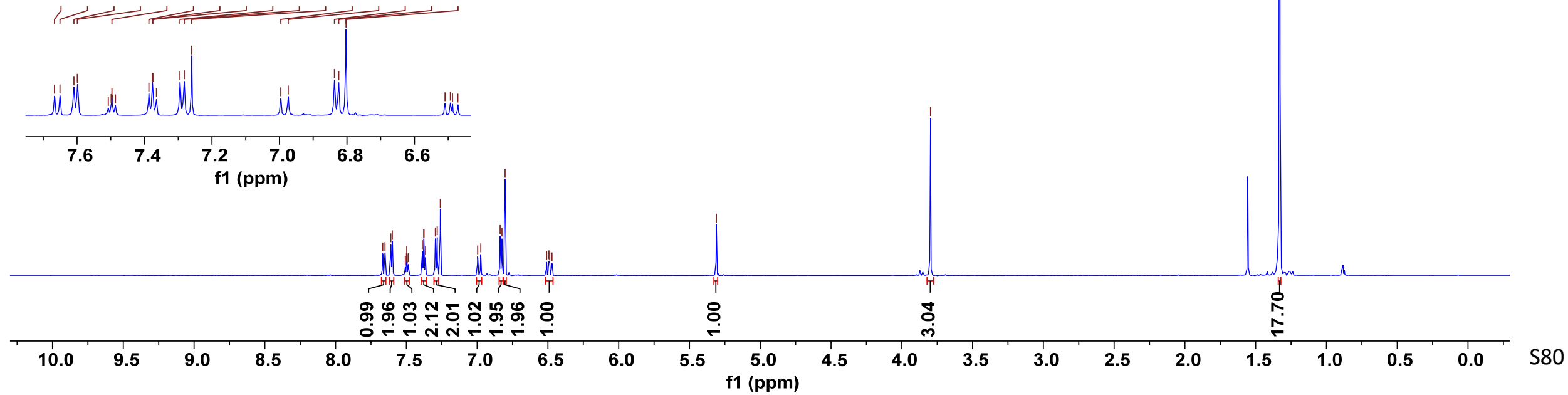


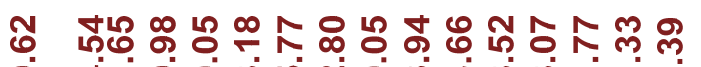

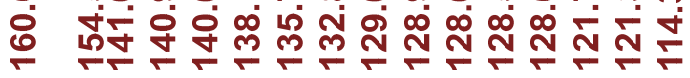

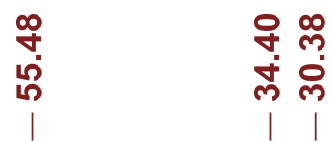

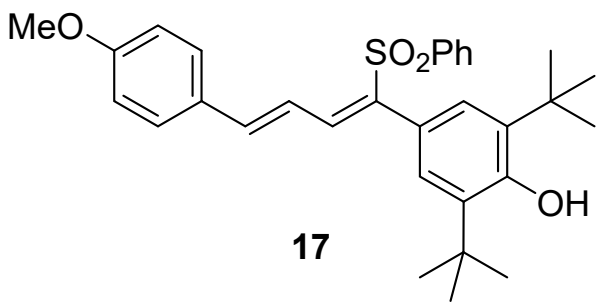

${ }^{13} \mathrm{C}$ NMR $\left(176 \mathrm{MHz}, \mathrm{CDCl}_{3}\right)$

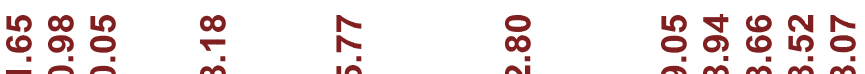

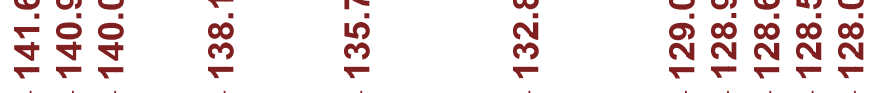

1,1 ।

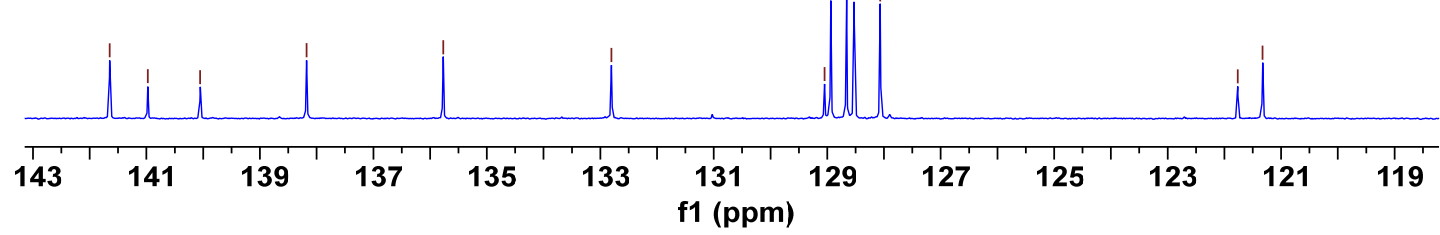


鱼 는 훙 m

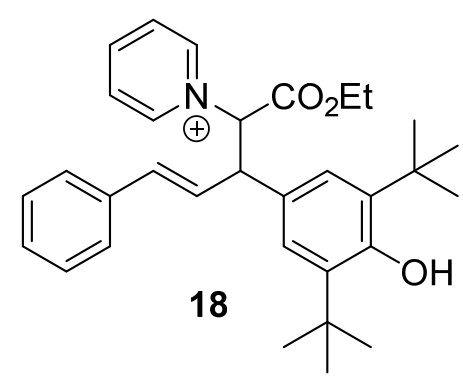

${ }^{1} \mathrm{H} \mathrm{NMR}\left(700 \mathrm{MHz}, \mathrm{CDCl}_{3}\right)$

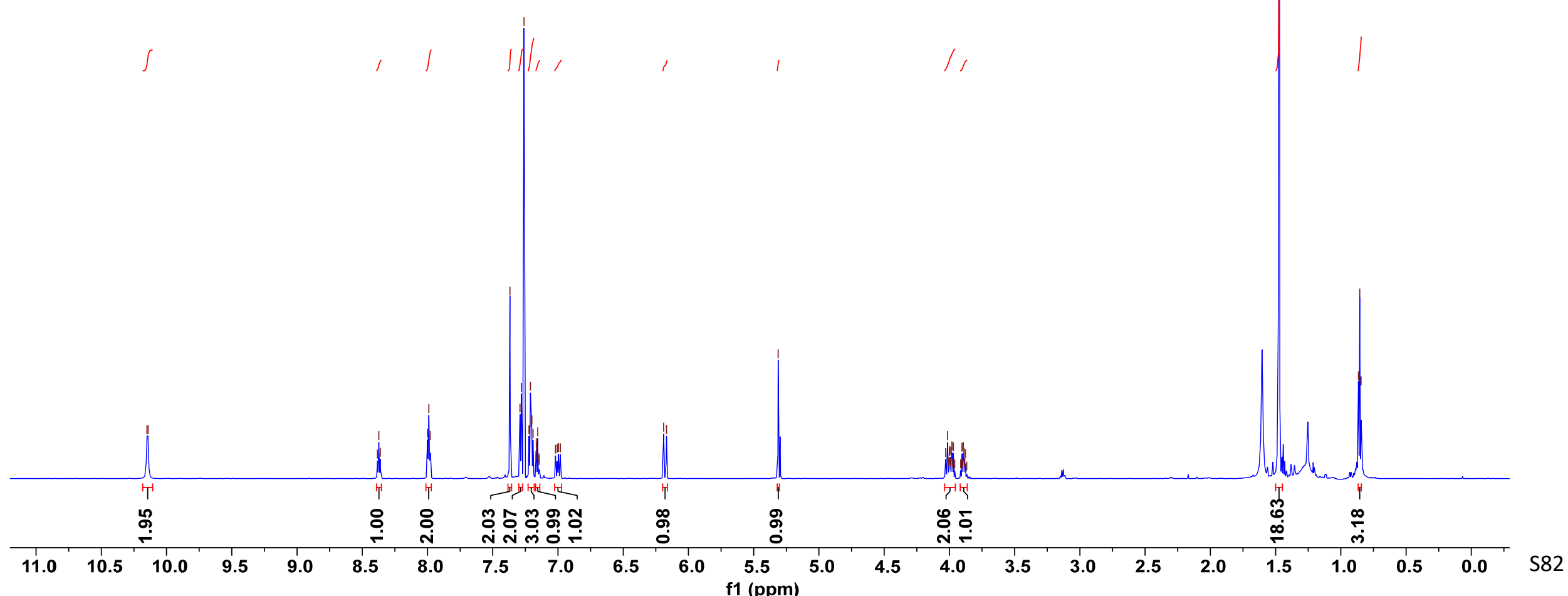




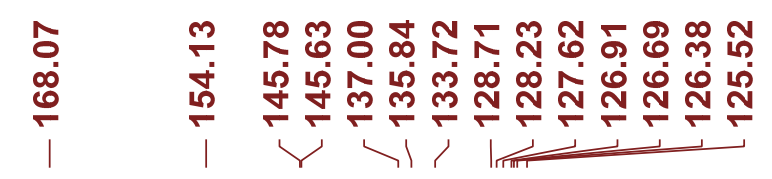

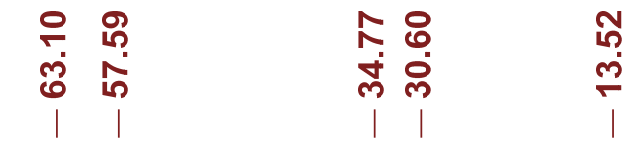

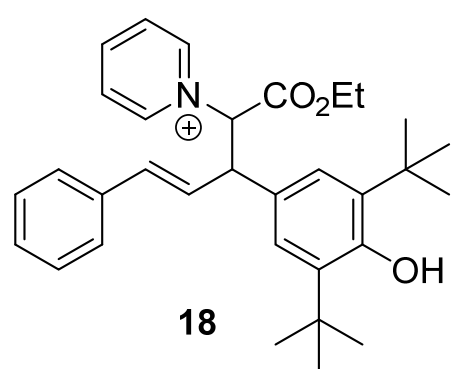

${ }^{13} \mathrm{C}$ NMR $\left(176 \mathrm{MHz}, \mathrm{CDCl}_{3}\right)$ 


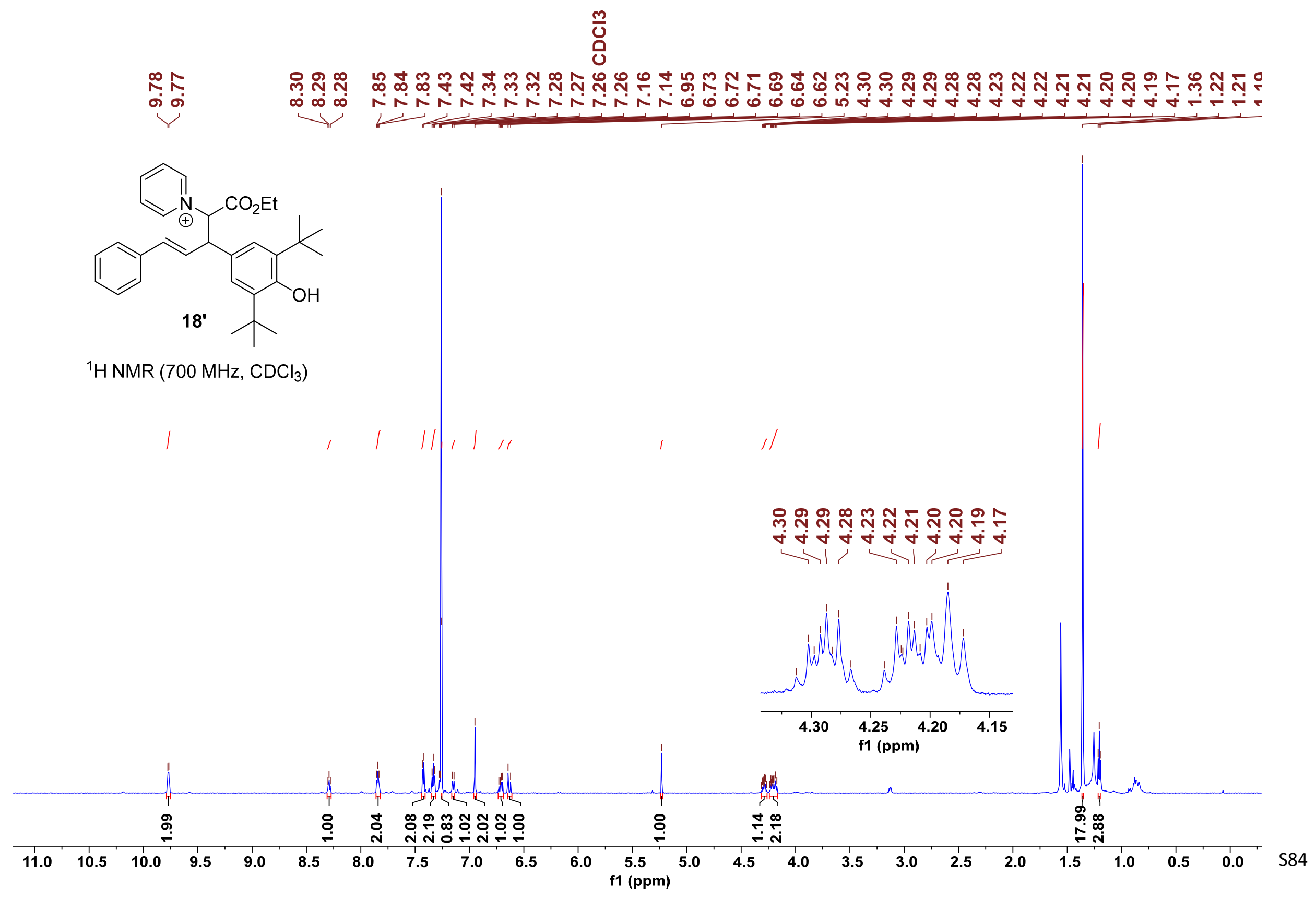




每

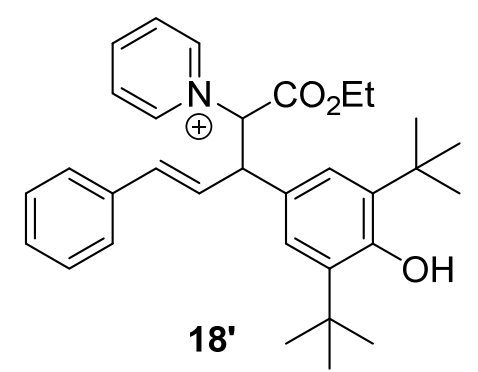

${ }^{13} \mathrm{C}$ NMR $\left(176 \mathrm{MHz}, \mathrm{CDCl}_{3}\right)$
జా

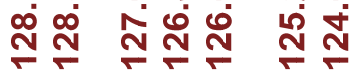

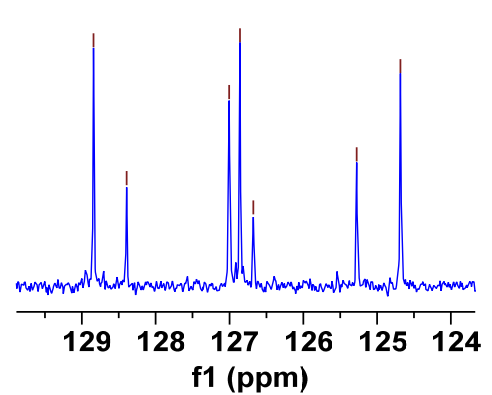


ํㅡㄴ

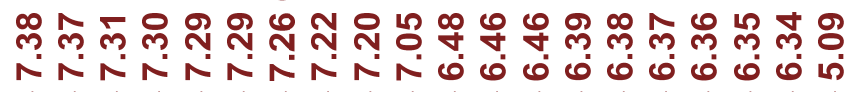

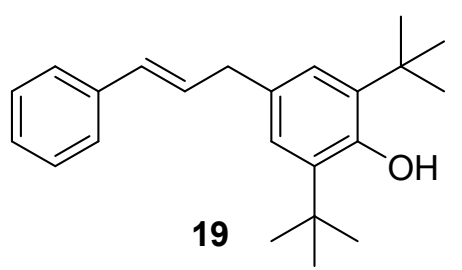

${ }^{1} \mathrm{H} \mathrm{NMR}\left(700 \mathrm{MHz}, \mathrm{CDCl}_{3}\right)$

$\frac{m}{0}$

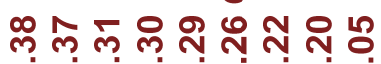

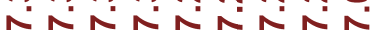
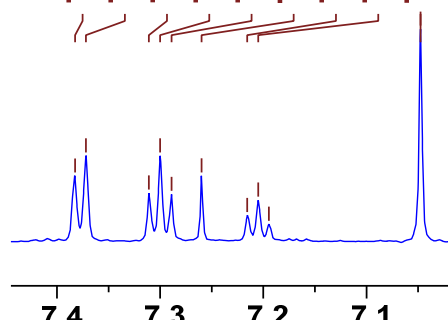

f1 (ppm)

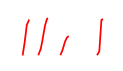

11

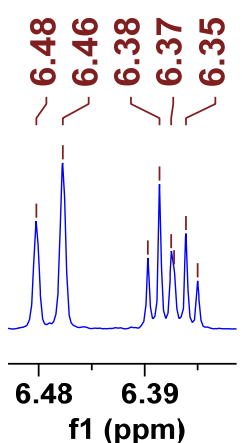

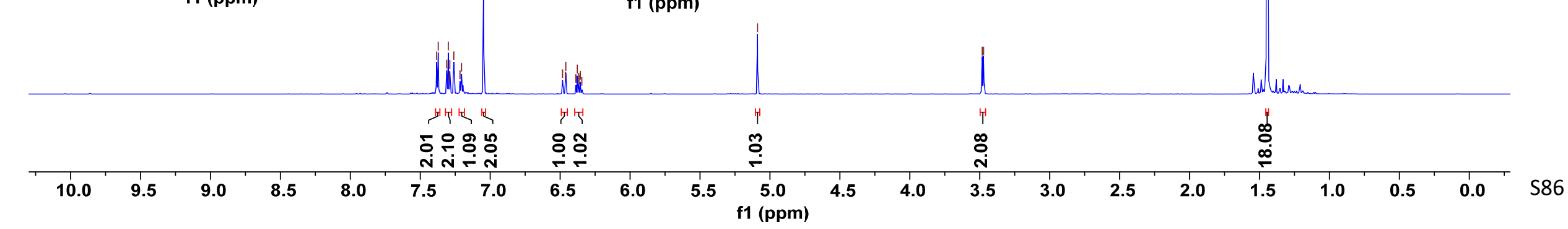




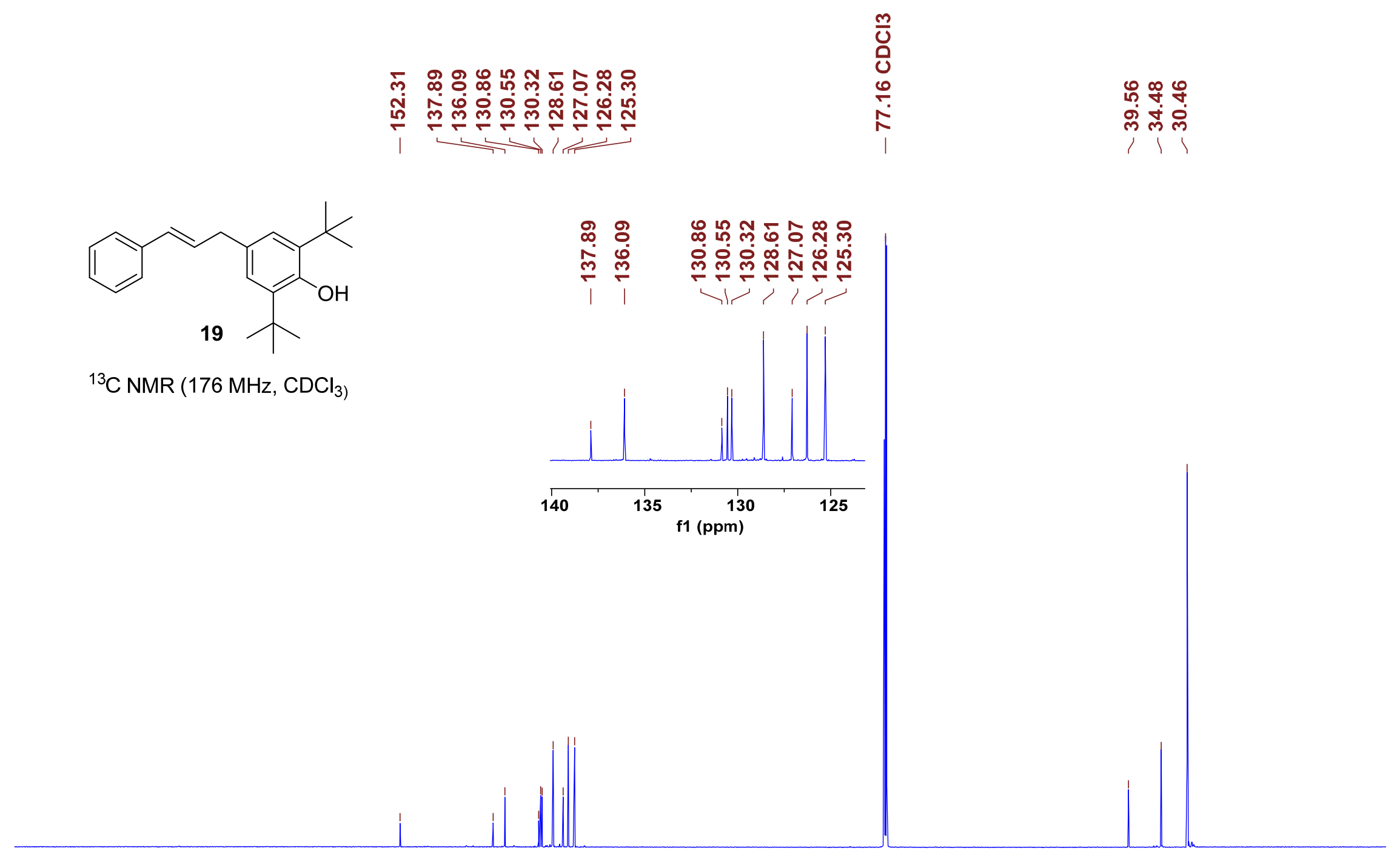

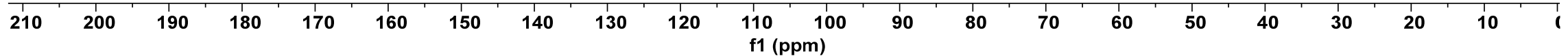


魚

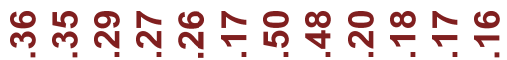

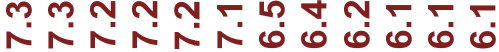
Cl

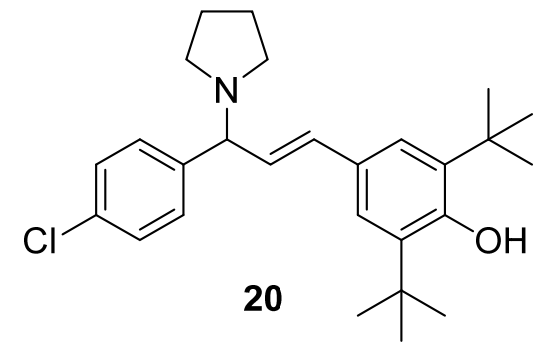

${ }^{1} \mathrm{H} \mathrm{NMR}\left(700 \mathrm{MHz}, \mathrm{CDCl}_{3}\right)$
ำ

1

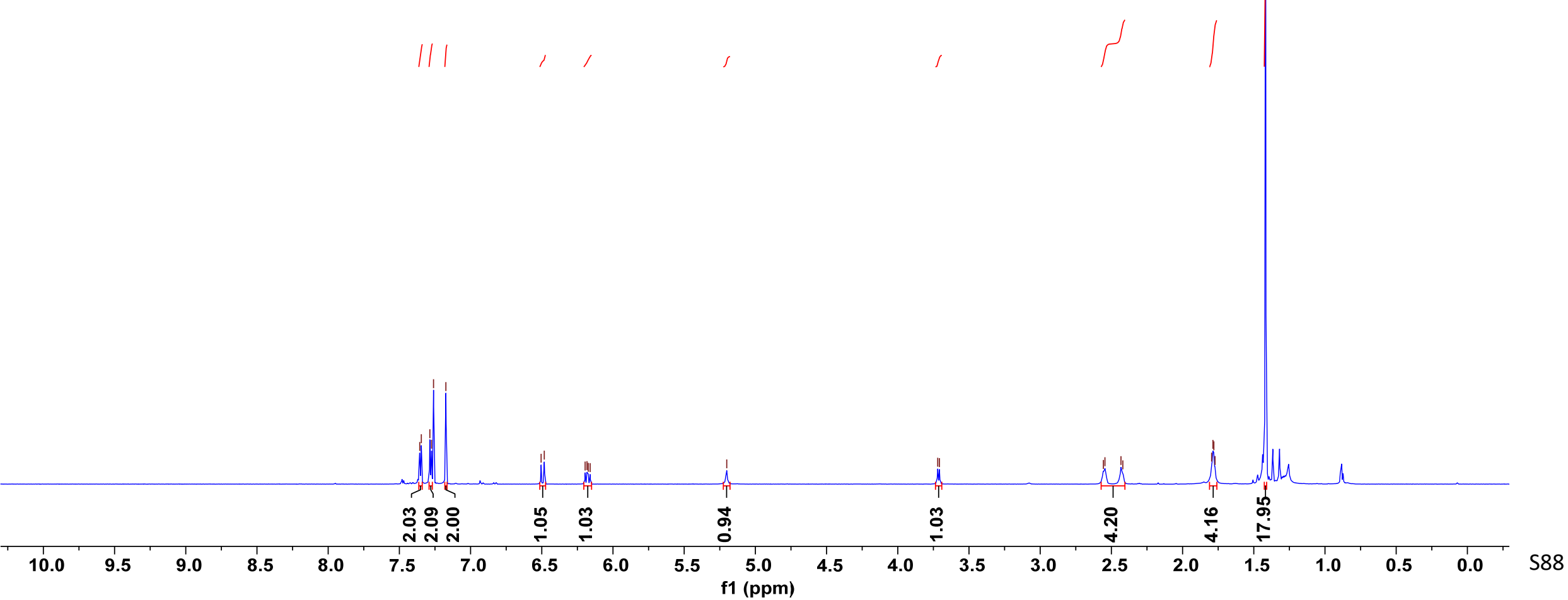




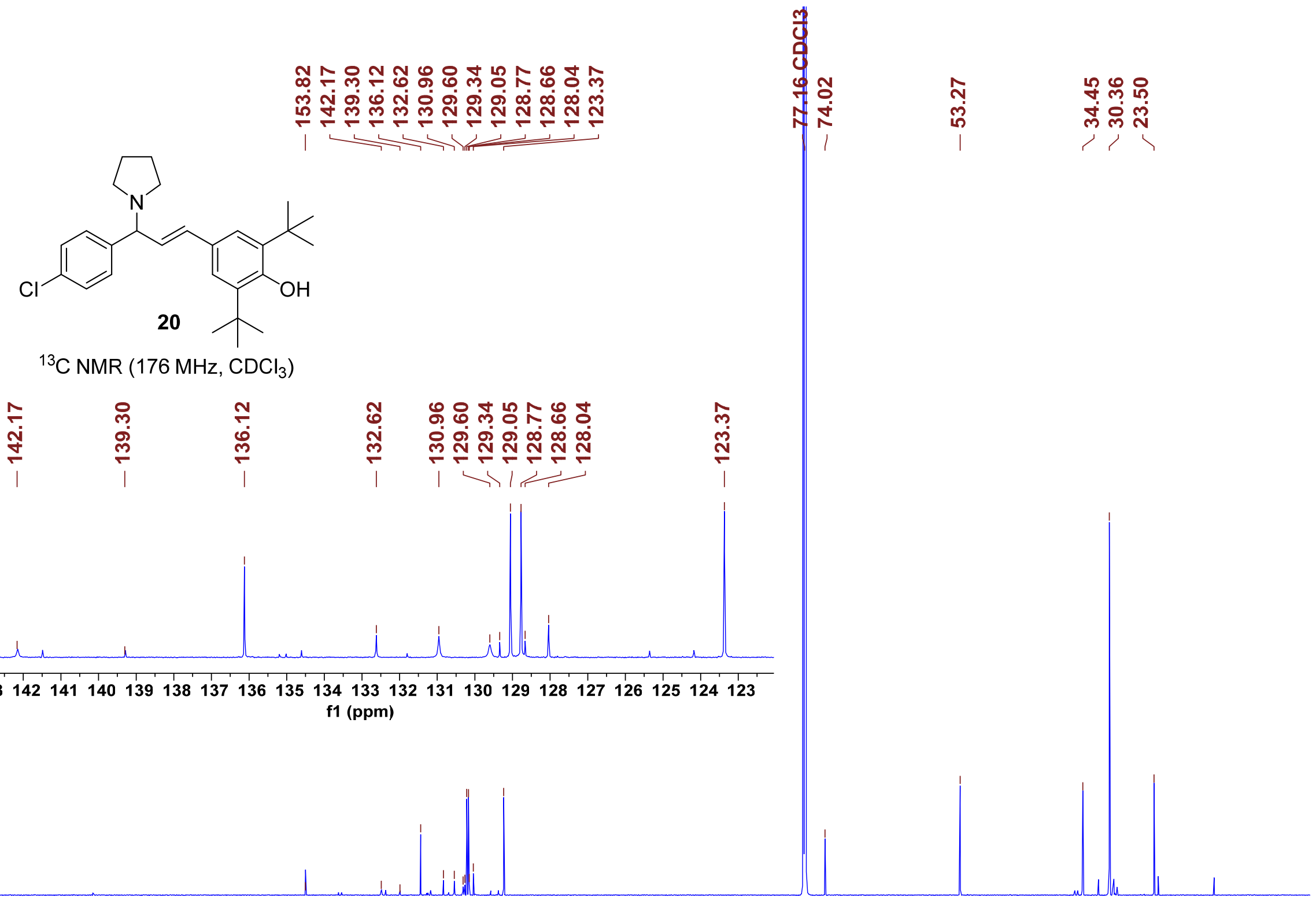

144143142141140139138137136135134133132131130129128127126125124123 f1 (ppm) 
鱼

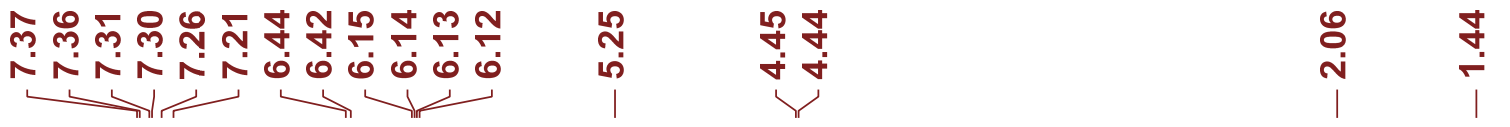

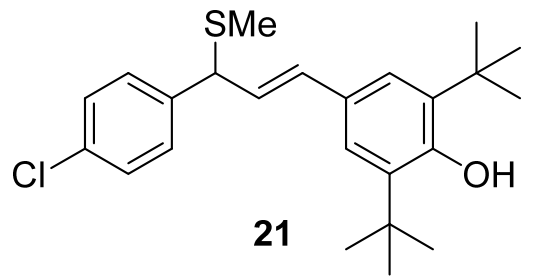

${ }^{1} \mathrm{H} \mathrm{NMR}\left(700 \mathrm{MHz}, \mathrm{CDCl}_{3}\right)$

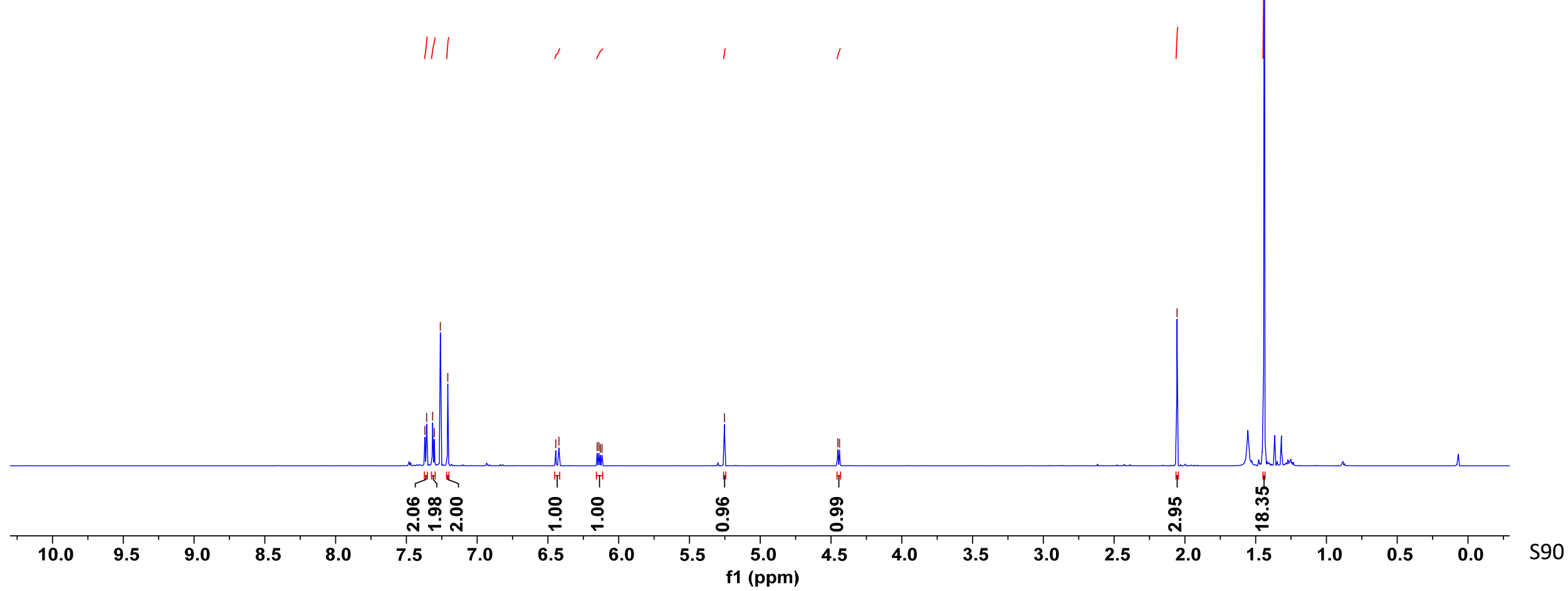


ᄂ

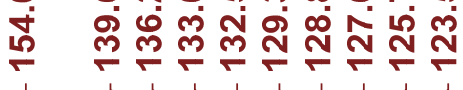

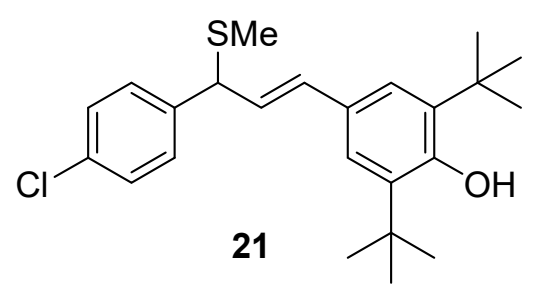

${ }^{13} \mathrm{C}$ NMR (176 MHz, $\mathrm{CDCl}_{3}$ ) 


\section{References}

(S1) Hansch, C.; Leo, A.; Hoekman, D. Exploring QSAR: Hydrophobic, Electronic, and Steric Constants; American Chemical Society: Washington, DC, 1995.

(S2) Fulmer, G. R.; Miller, A. J. M.; Sherden, N. H.; Gottlieb, H. E.; Nudelman, A.; Stoltz, B. M.; Bercaw, J. E.; Goldberg, K. I. Organometallics 2010, 29, 2176-2179.

(S3) Aranzaes, J. M.; Daniel, M.-C.; Astruc, D. Can. J. Chem. 2006, 84, 288-299.

(S4) SAINT; Bruker AXS Inc.: Madison, Wisconsin, USA, 2012.

(S5) Sheldrick, G. M. SADABS; University of Göttingen, Germany, 1996.

(S6) Sheldrick, G. M. Acta Cryst. A 2015, 71, 3-8.

(S7) (a) Zhang, X.-Z.; Deng, Y.-H.; Gan, K.-J.; Y., X.; Yu, K.-Y.; Wang, F.-X.; Fan, C.-A. Org. Lett. 2017, 19, 1752-1755. (b) Yuan, Z.; Gai, K.; Wu, Y.; Wu, J.; Lin, A.; Yao, H. Chem. Commun. 2017, $53,3485-3488$.

(S8) Harder, E.; Damm, W.; Maple, J.; Wu, C.; Reboul, M.; Xiang, J. Y.; Wang, L.; Lupyan, D.; Dahlgren, M. K.; Knight, J. L.; Kaus, J. W.; Cerutti, D. S.; Krilov, G.; Jorgensen, W. L.; Abel, R.; Friesner, R. A. J. Chem. Theory Comput. 2016, 12, 281-296.

(S9) Schrödinger Release 2019-1: MacroModel, Schrödinger, LLC, New York, NY, 2019.

(S10) Rocha, G. B.; Freire, R. O.; Simas, A. M.; Stewart, J. J. P. J. Comput. Chem. 2006, 27, 1101-1111.

(S11) Zhao, Y.; Truhlar, D. G. Theor. Chem. Acc. 2008, 120, 215-241.

(S12) Marenich, A. V.; Cramer, C. J.; Truhlar, D. G. J. Phys. Chem. B 2009, 113, 6378-6396.

(S13) Gaussian 16, Revision A.03, Frisch, M. J.; Trucks, G. W.; Schlegel, H. B.; Scuseria, G. E.; Robb, M. A.; Cheeseman, J. R.; Scalmani, G.; Barone, V.; Petersson, G. A.; Nakatsuji, H.; Li, X.; Caricato, M.; Marenich, A. V.; Bloino, J.; Janesko, B. G.; Gomperts, R.; Mennucci, B.; Hratchian, H. P.; Ortiz, J. V.; Izmaylov, A. F.; Sonnenberg, J. L.; Williams-Young, D.; Ding, F.; Lipparini, F.; Egidi, F.; Goings, J.; Peng, B.; Petrone, A.; Henderson, T.; Ranasinghe, D.; Zakrzewski, V. G.; Gao, J.; Rega, N.; Zheng, G.; Liang, W.; Hada, M.; Ehara, M.; Toyota, K.; Fukuda, R.; Hasegawa, J.; Ishida, M.; Nakajima, T.; Honda, Y.; Kitao, O.; Nakai, H.; Vreven, T.; Throssell, K.; Montgomery, J. A., Jr.; Peralta, J. E.; Ogliaro, F.; Bearpark, M. J.; Heyd, J. J.; Brothers, E. N.; Kudin, K. N.; Staroverov, V. N.; Keith, T. A.; Kobayashi, R.; Normand, J.; Raghavachari, K.; Rendell, A. P.; Burant, J. C.; lyengar, S. S.; Tomasi, J.; Cossi, M.; Millam, J. M.; Klene, M.; Adamo, C.; Cammi, R.; Ochterski, J. W.; Martin, R. L.; Morokuma, K.; Farkas, O.; Foresman, J. B.; Fox, D. J. Gaussian, Inc., Wallingford CT, 2016. 